\title{
A Force-controlled Indenter to Elicit a Tactile Afferent Response that Accommodates for the Skin's Viscoelastic Relaxation and Differing Thickness
}

\author{
A Thesis \\ Presented to \\ the faculty of the School of Engineering and Applied Science \\ University of Virginia \\ In partial fulfillment \\ of the requirements for the degree \\ Master of Science
}

by

Sean Lawrence Gallahan

May

2015 
Approval Sheet

The thesis

is submitted in partial fulfillment of the requirements

for the degree of

Master of Science

Author

The thesis has been read and approved by the examining committee:

Gregory Gerling, Ph.D.

Advisor

Amy Laviers, Ph.D.

Reid Bailey, Ph.D.

Ellen Lumpkin, Ph.D.

Accepted for the School of Engineering and Applied Sciences:

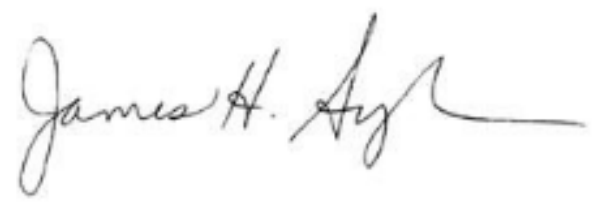

Dean, School of Engineering and Applied Science

May

2015 


\section{TABLE OF CONTENTS}

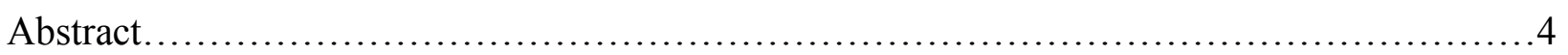

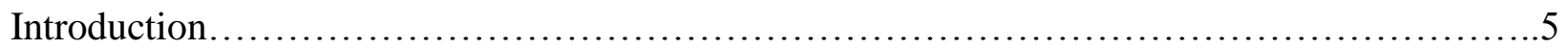

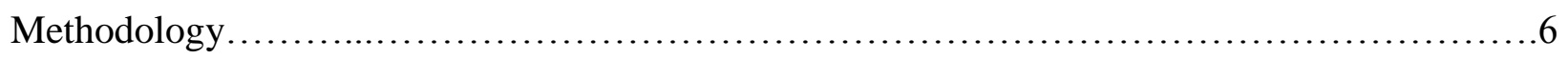

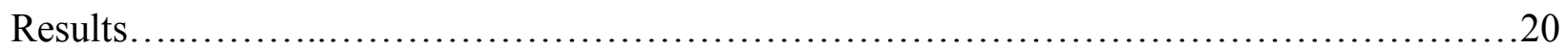

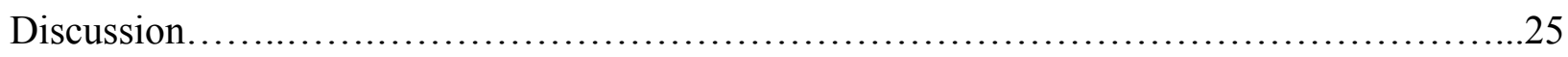

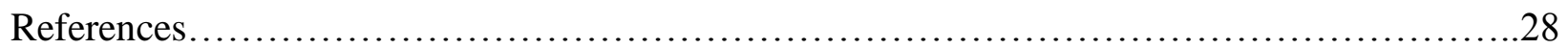

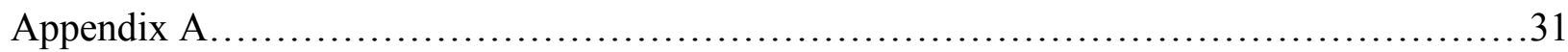

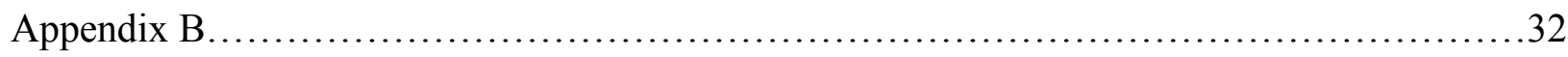

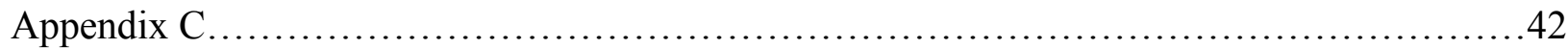

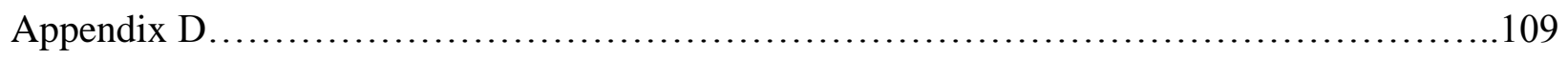




\section{Abstract}

Tactile afferents elicit neural biopotentials upon deformation of the skin's surface. To study the underlying input-output relationship, typically the stimulus input is displacement, though force may be more naturalistic. Force control is advantageous because equal pressure can be delivered to specimens that vary in skin thickness and it can counterbalance the skin's viscoelastic relaxation. However, current force-controlled indenters approach an afferent's receptive field at an angle so as to create an unequal stress distribution and base their feedback control indirectly on motor performance as opposed to directly measuring probe force. The proposed research seeks to address these issues by designing, building, and evaluating a singleaxis indenter. The indenter consists of hardware (motion controller coupled with a mechanical sled and custom designed electronics) and software (control algorithm and user interface). The control algorithm utilizes a sequential, pre-planned trajectory and then a real-time feedback technique to counterbalance the three phases of skin relaxation that accompany the early hold (< $.19 \mathrm{sec})$, intermediate hold $(>\sim 0.19 \mathrm{sec}$ and $<\sim 1.5 \mathrm{sec})$ and late hold $(>\sim 1.5 \mathrm{sec})$ of the stimulus. The graphical user interface (Python software) traverses a user intuitively through a series of experimental tasks. With collaborators at Columbia University, we conducted two experiments. The results of the experiments indicate the indenter 1) can clamp an achieved force in skins of differing thickness and 2) evoke neurophysiological responses of slowly adapting afferents whereby the firing rate drop between the early and late phases of the sustained hold is less during force control, leaving mostly neural adaptation. 


\section{Introduction}

Our sense of touch is informed by the responses of tactile afferents embedded in the skin. The patterns of spiking response differ according to afferent type (Johnson, 2001). For example, slowly adapting afferents will respond to both moving ramp and sustained hold phases of a stimulus. In experiments, what is typically held sustained is the displacement, or position. This is displacement control. Two phenomena are observed when displacement control is employed; the skin rapidly decays at a double exponential rate (at least) during the static phase (Williams et. al, 2010 and Pubols and Benkich, 1986) and differences in skin thickness can lead to unequally delivered sets of stimuli (Wang et al, 2013). Given the first phenomena, it is hard to decouple if the observed decay or adaptation in the spike firing of the neural response is due to skin relaxation or neuronal mechanisms. Given the second, changes in skin thickness of $10 \mathrm{~s}$ to $100 \mathrm{~s}$ of microns between specimens means that the same set of displacements will elicit a different sensitivity function, which does not allow for accurate cross comparison (Wang et al, 2013). Furthermore, it is unclear whether humans (and other mammals) actively attempt to control force over displacement, but force control seems more likely (Srinivasan and Lamonte, 1995 and Tiest and Kappers, 2009).

At present, there are few compressive, force controlled indenters on the market. One offthe-shelf model, the 300 series (Aurora Scientific Inc, Canada) was designed for the application of tension to muscles, but has also been configured for compression to the tactile end organs (Jang, 2009 and Levy and Strassman, 2002). While this indenter has a ramp up time of less than $200 \mathrm{msec}$ at the lowest velocity setting and can switch between force and displacement control, its rotary lever mechanism can create an unequal state of stress at the skin surface. Secondly, there is little documentation on the readout or accuracy of its force clamp, and its feedback 
control is based indirectly on motor performance as opposed to direct measurement of force at the probe tip. Another alternative force control indenter has likewise not reported the real-time measurement of the force clamp, and has typically been used at larger forces around and above 4 Newtons (Johansson, 2004).

To address the aforementioned issues of skin phenomena and other equipment, this work focuses upon the design, construction, and evaluation of a compressive force-controlled indenter.

\section{Methodology}

The Virginia force-controlled indenter was built to deliver ramp and hold stimuli to afferent end organs of the skin. The design metrics include delivering forces over the range of 1 $-2500 \mathrm{mN}$ with a $1.5 \mathrm{~mm}$ tip, at ramp durations of at or less than $600 \mathrm{msec}$ to $100 \mathrm{mN}$ force, and achieving force clamps with error of less than $5 \%$ of that achieved. Off-the-shelf and custom-built components were used, and major sub-systems include (as described in Section 3.2.): Actuators, Mounts and Sensors, Control Algorithms, User Controls and Displays, and Integration and Electronics Interfacing.

As indicated in Fig. 1, a series of transformations are inherent in the indenter's operation. Displacements and/or forces are commanded at the graphical user interface. Control algorithms work with the motion controller to move the vertical sled. The position of the probe tip at a cantilever attached the vertical sled is monitored by laser displacement sensor and load cell and recorded by an analog-to-digital converter. In force-control mode, probe measurements are relayed back to the control algorithms and motion controller for position updates of the vertical sled, to accommodate for skin relaxation and maintain constant force. The force control algorithm splits into two parts: a pre-programmed trajectory to reach the desired force and clamp 
the skin's early decay and feedback control to counteract the skin's later decay. The extremely rapid and large magnitude of the skin's relaxation (described in detail in Section 3.1) calls for the utilization of the pre-programmed trajectory.

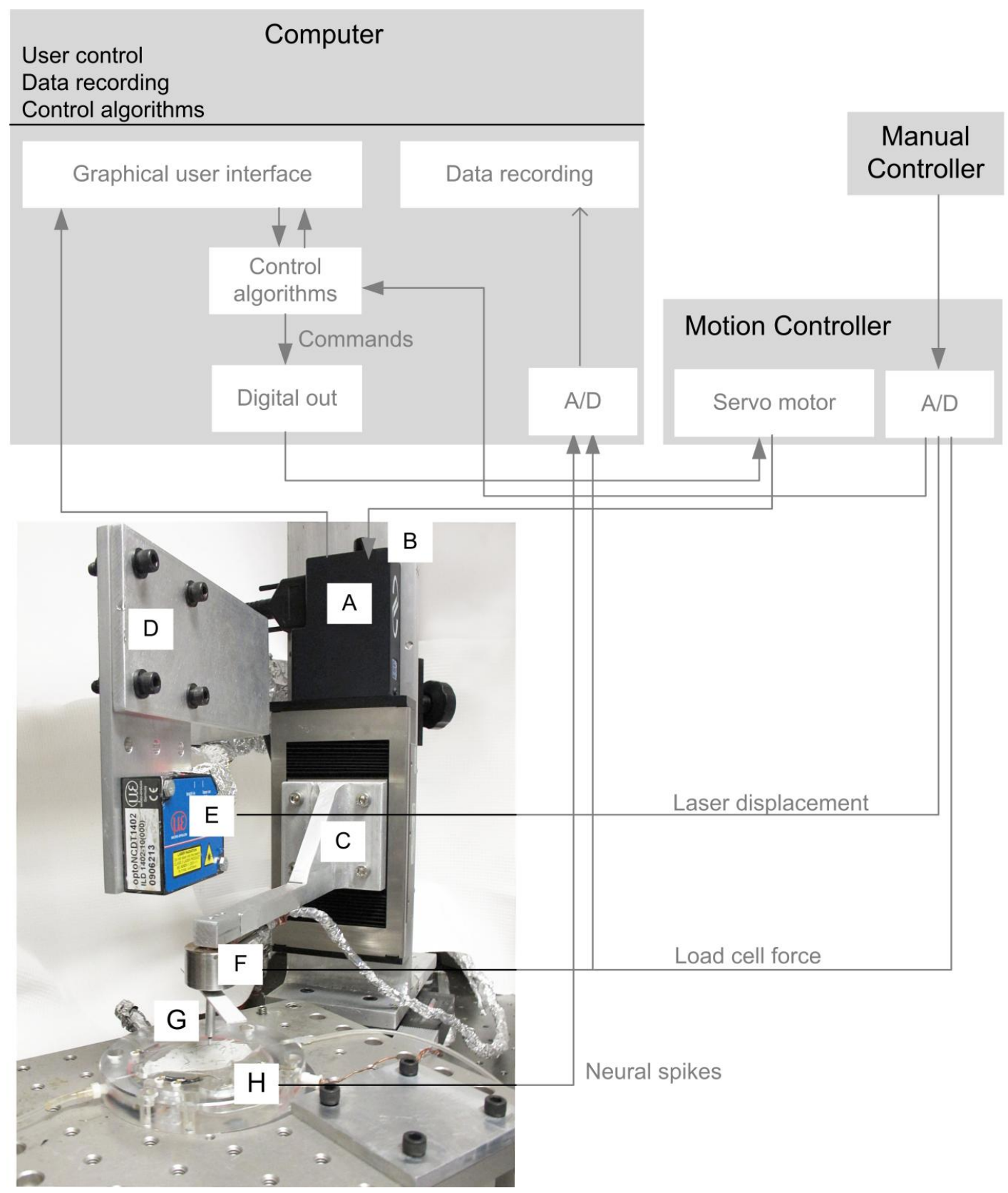

Figure 1: Components of and data flow between the indenter's sub-systems. In particular, the actuators, sensors and mount consist of a motion sled (A), t-beam (B), cantilever (C), laser cantilever and height adjust (D), laser (E), load cell (F), magnetic base, tip (G), and prep stage and voltage reading equipment (H). 
To test the indenter's performance, two sets of experiments were run. Experiment 1 compared displacement- and force-controlled indentations into mouse skin of differing thickness, from two animals with three body sites, at a ramp velocity of $0.5 \mathrm{~mm} / \mathrm{secec}$. Experiment 2 examined an ex vivo neural recording from a slowly adapting fiber in the skin of one mouse.

\subsection{Exploration into force decay as a result of skin relaxation}

An understanding of both the magnitude and timing of the skin's relaxation is a vital step to precede design. As such, force versus time data for twenty-one indentations into ex vivo mouse skin, across five specimens, were conducted as described in Wellnitz (2010). The skin was pinned on top of a silicone elastomer (Sylgard, Dow Corning) and nylon (L'eggs winterweight pantyhose). Indentations spanned across a range of peak forces, from 66 to $660 \mathrm{mN}$ with a $1.5 \mathrm{~mm}$ tip. These displacement-controlled experiments used ramp up acceleration of 6.35 $\mathrm{mm} / \mathrm{sec}^{2}$ and velocity of $0.5 \mathrm{~mm} / \mathrm{sec}$. The results indicate that the skin's relaxation best fits a triple exponential function where the medians of the three time constants, across the twenty-one indentations, are 189, 1050, 136,119 msec, respectively. One example indentation and fitting is shown in Fig. 2. The results also demonstrate that as peak force increases, the time constants $(\tau)$ also increase. Based on these findings, we determined that the indenter, in feedback control mode only, with a timing cycle of 20-30 msec per movement as well as the need to stop between each movement as a result of the controller's sigmoidal displacements, could not keep up with the magnitude of the skin's rapid decay. Therefore, we developed a pre-programmed trajectory method to clamp this rapid early decay. 

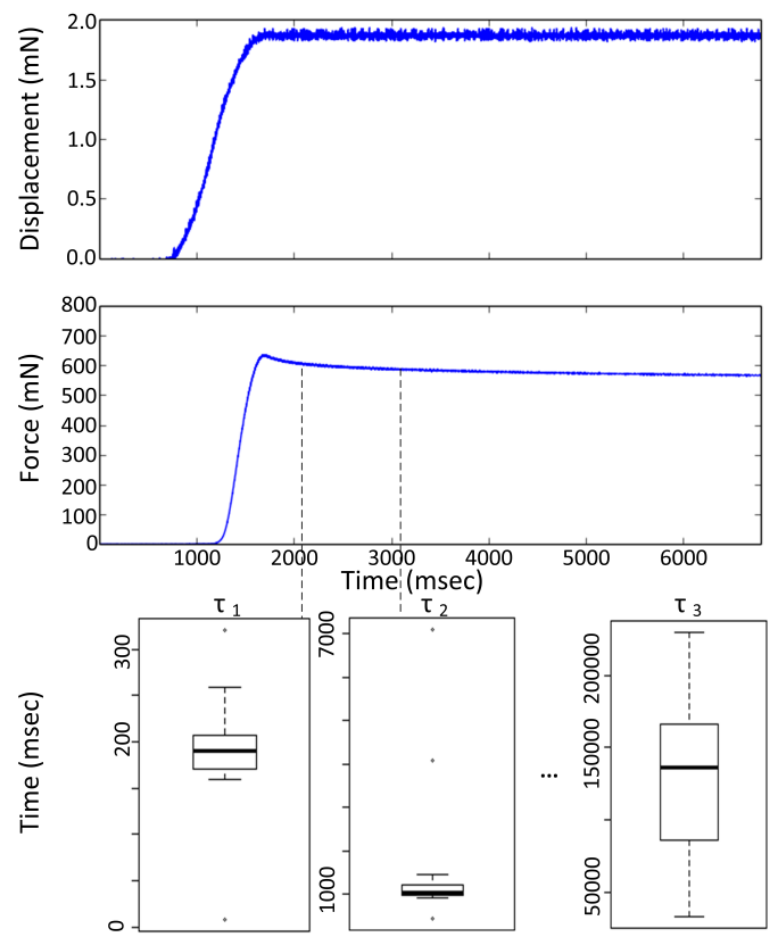

Figure 2: An example of the skin's relaxation under displacement control. The upper trace shows that displacement is clamped to $1.9 \mathrm{~mm}$ resulting in a peak force of about $660 \mathrm{mN}$ in the middle trace, which decays in the steady-state to about $14 \%$ of the peak force. Triple exponential functions were fitted to force traces, starting at their peak force, over magnitudes from $66 \mathrm{mN}$ to $660 \mathrm{mN}$. For example, when fit to a triple exponential function, the force trace above yields time constants of 255,1470 , and 229,156 msec. The time constant $\tau_{3}$ is not annotated in the figure because it extends well past the 5 second hold period.

\subsection{Apparatus}

\section{Actuators, Mount, and Sensors}

Actuators, sensors and mount components respond to commands from the motion controller, monitor the sensors (e.g., laser and load cell), and fasten the actuators and sensors to the Faraday cage on a vibration free table. A single $50 \mathrm{~mm}$ length DC motor driven sled (50 cc UTS Mid-Travel Steel Linear Stages, Newport Corporation, USA) drives the indenter tip in the z-direction into the skin prep (Fig. 1, mark A). The sled is constrained to a maximum velocity of $40 \mathrm{~mm} / \mathrm{sec}$, a maximum acceleration of $160 \mathrm{~mm} / \mathrm{sec}^{2}$ and a minimum movement of 0.3 micrometers. Two x- and y- direction actuated stages (100 mm travel, Siskiyou Inc., Oregon), 
oriented perpendicular to each other, position the probe tip lateral to the surface of the prep between experiments.

For stability, the sled is held upright by an aluminum t-beam (length $=92.0 \mathrm{~mm}$, width $=$ $63.5 \mathrm{~mm}$, height $=381.0 \mathrm{~mm})$ welded to an aluminum base $($ length $=177.8 \mathrm{~mm}$, width $=101.6$ $\mathrm{mm}$, height $=12.7 \mathrm{~mm})($ Fig. 1 , mark B; and Appendix B $)$. The base of the t-beam connects directly to the $\mathrm{x}-\mathrm{y}$ stages and sit atop a low profile magnetic base with a $300 \mathrm{~N}$ vertical maximum force threshold (Thorlabs, New Jersey). Attached perpendicular to the sled's moving platform is a lightweight, aluminum cantilever arm (length $=190.5 \mathrm{~mm}$, width $=12.7 \mathrm{~mm}$, height $=12.7$ $\mathrm{mm}$ to $76.2 \mathrm{~mm}$, base $=75.0$ by $75.0 \mathrm{~mm})($ Fig. 1 , mark C).

A load cell (Model 31, Honeywell, USA), aluminum sheath and ceramic tip (Fig. 1, mark F and mark G) are positioned at the end of the cantilever arm. The load cell operates within a maximum load of 250 grams ( $\sim 2.5$ Newtons) with a resolution of $+-0.5 \mathrm{mN}$ and outputs analog voltage linearly correlated to force. The ceramic tip (10.0 mm length, $1.6 \mathrm{~mm}$ diameter), with a filleted end, connects to the load cell via an aluminum sheath $(22.5 \mathrm{~mm}$ length, $3.2 \mathrm{~mm}$ diameter). The aluminum sheath and ceramic tip were carefully designed to limit vibrational noise through reduction of mass and volume and collectively weigh 4.1 grams. Located on the back of the base is a large steel shaft that connects to a holster and angled beam to hold the displacement laser (OptoNCDT1402, Micro-Epsilon, Germany) with a $10.0 \mathrm{~mm}$ range and 1.0 um resolution directly over top of a small rectangular piece of aluminum and measures displacement of the ceramic tip (Fig. 1, mark E). 


\subsubsection{Control Algorithms}

The control algorithms command those displacements, forces, velocities and accelerations specified at the user interface, send commands to the motion controller, and receive data from sensors. The mechanical indenter employs separate algorithms for displacement and force control.

\subsubsection{Displacement Control}

Under displacement control (Fig. 2, top), the probe tip is commanded to a position into the skin relative to their point of contact. The three input variables are displacement, velocity, and hold duration.

\subsubsection{Force Control}

Under force control, the sled is commanded to a force and then achieved force is measured and fed back to the algorithm by the load cell. To accomplish the force clamp, the algorithm utilizes two temporally successive tables concatenated into a single trajectory, and a PID feedback loop (Fig. 3). A trajectory is a pre-planned sequence of positions by which to move the tip over time and at a certain rates. The trajectory consists of two separate components: 1) over the ramp up ( $600 \mathrm{msec}$ duration), a look up table to move to a displacement paired to a commanded force and 2) over the first and second time constant of the triple exponential decay (from start of force clamp to $\sim 1.5 \mathrm{~s}$ ), a series of discrete movements every $1 \mathrm{~ms}$ to clamp the force. The trajectories are populated via a series of calibrations (described below) that correspond to levels of force. 


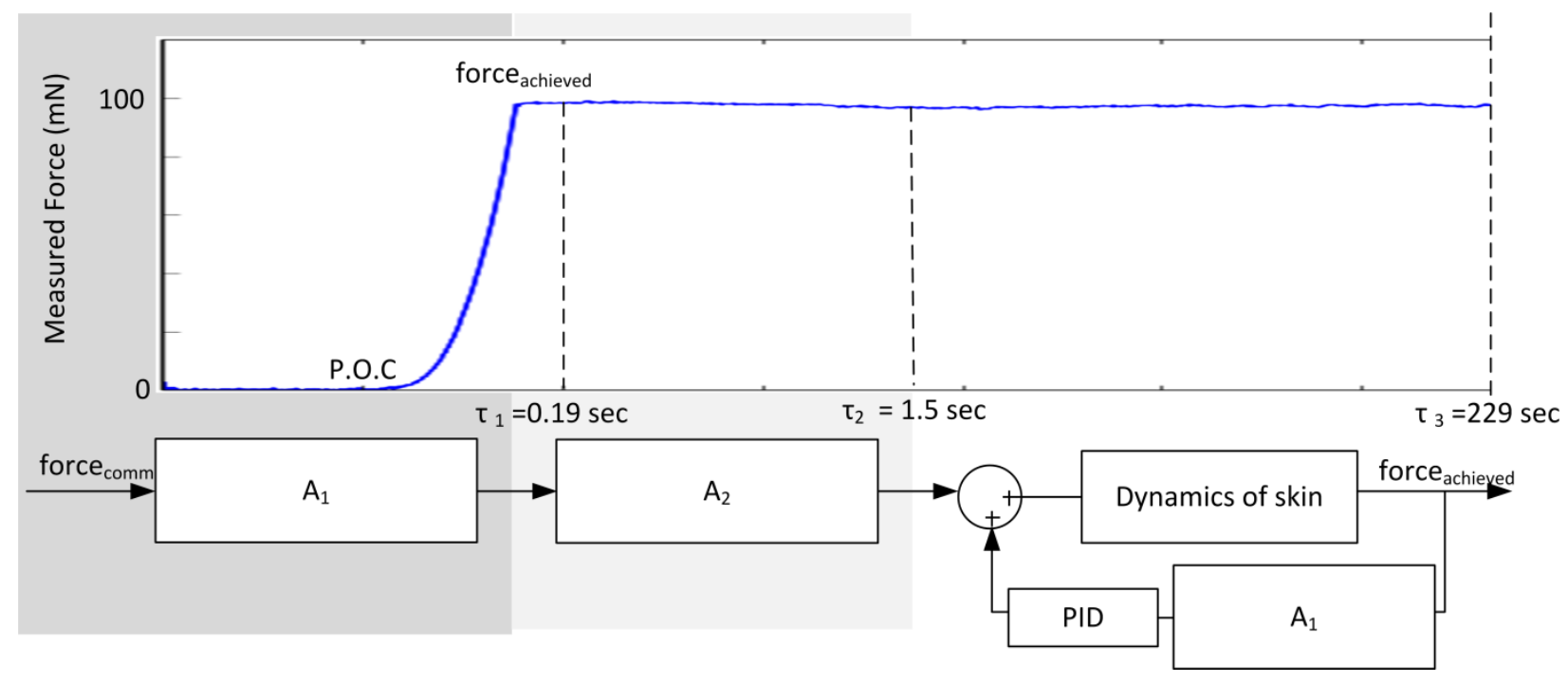

Figure 3: The force control strategy involves three main steps. The first two steps consist of a pre-planned trajectory based on prior calibration experiments. In particular, the $A_{1}$ table is a force-to-displacement lookup that accounts for the displacements neeeded over the course of the stimulus ramp to achieve a desired peak force. The $A_{1}$ table is built up as indicated in Fig. 4. In addition to its use in guiding motion over the course of the ramp, it is also used to help build the $A_{2}$ look-up table. Then, the $A_{2}$ table is a second force-todisplacment look-up table that accounts for the displacements needed from the end of the $A_{1}$ table's ramp phase until about $\tau_{2}=1.5$ seconds, a second set of displacements are configured, annotated. The $A_{2}$ table is built up as indicated in Fig. 5. The third step is a PID feedback loop that converts the error in force back into a displacement to counteract the slower decay late in the hold. The feedback loop runs until the commanded hold time ends, which may be longer or shorter then $\tau_{3}$.

The PID feedback loop, following the completion of the trajectory, controls the force clamp to the end of the hold, adjusting for less skin relaxation during the third exponential. In particular, the algorithm adjusts to the current load cell measurement, as the current force may be above or below commanded force. PID multiplication values, represented in Eqns. 1, 2 and 3 with 'mult' subscripts, were found during experimentation into mouse skin on top of sylgard (description in Wang, 2013). Note that dt varies depending on the loop time of each feedback iteration. Accurate $\mathrm{P}_{\text {mult }}, \mathrm{I}_{\text {mult }}$, and $\mathrm{D}_{\text {mult }}$ vary greatly based on skin thickness, and the interface allows the user to adjust PID values according to perceived skin thickness, via a continuous slider with roughly three levels of 'thin' $\left(\mathrm{P}_{\text {mult }}=.0005, \mathrm{D}_{\text {mult }}=.000005, \mathrm{I}_{\text {mult }}=.0005\right)$, 'normal' $\left(\mathrm{P}_{\text {mult }}=.005, \mathrm{D}_{\text {mult }}=.00005, \mathrm{I}_{\text {mult }}=.0005\right)$ and 'thick' $\left(\mathrm{P}_{\text {mult }}=.05, \mathrm{D}_{\text {mult }}=.0005, \mathrm{I}_{\text {mult }}=.0005\right)$.

These functions then combine to be sled movement, Eqn. 4, to adjust for the decay. 
$\mathrm{P}_{\text {error }}=\mathrm{P}_{\text {mult }} *$ error $_{\text {current }}$

$\mathrm{I}_{\text {error }}=\mathrm{I}_{\text {mult }} *$ Integrator $\left(\right.$ Integrator $=$ Integrator $_{\text {prev }}+$ Integrator $\left.^{*} \mathrm{dt}\right)$

$\mathrm{D}_{\text {error }}=\mathrm{D}_{\text {mult }} *\left(\right.$ error $_{\text {current }}-$ error $\left._{\text {prev }}\right) / \mathrm{dt}$

Movement $(\mathrm{mm})=\mathrm{P}_{\text {error }}+\mathrm{I}_{\text {error }}+\mathrm{D}_{\text {error }}$

\subsection{Calibration}

A two-step calibration procedure is used to create a trajectory. The first step corresponds to the $\mathrm{A}_{1}$ force-to-displacement table that accounts for movements over the stimulus ramp, and the second step corresponds to the $\mathrm{A}_{2}$ force-to-displacement table that accounts for movements to counteract skin relaxation over the stimulus hold. In particular, for the first step of calibration, the $A_{1}$ force-to-displacement table, displacement from the motion controller and force from the load cell (e.g., $d_{\max }=0.18 \mathrm{~mm}$ to $\mathrm{f}_{\max }=50 \mathrm{mN}$ ) at a rate of $10 \mathrm{kHz}$ are coupled to form a series of relationships over the duration of the stimulus ramp. This series of force and displacement relationships are fitted by an exponential function (Fig. 4, $\mathrm{A}_{1}$ : eq. 5), which will in later steps convert fitted force decay back to displacement.

Force $(\mathrm{mN})=\mathrm{a} e^{b * \operatorname{displacement}(\mathrm{mm})}+\mathrm{c}$ 


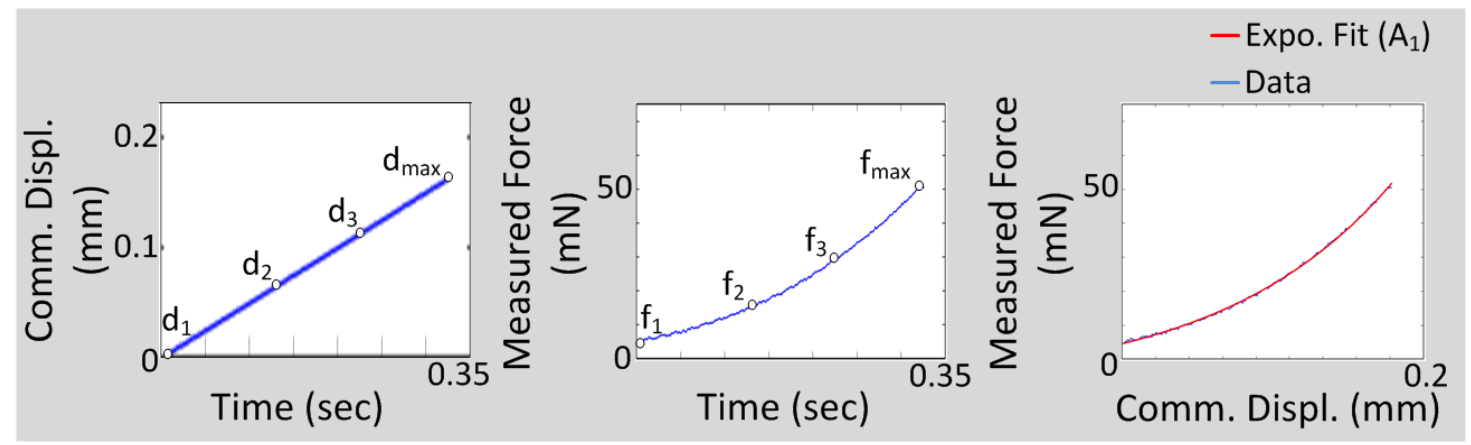

Figure 4: To build up the $A_{1}$ force-to-displacement table that accounts for movements over the stimulus ramp, a calibration stimulus is indented into the skin at a slow commanded velocity from the point of contact to a commanded force $\left(f_{\max }\right)$. When at the commanded force, the corresponding displacement is then saved as $d_{\text {max. }}$ During stimulation up to $f_{\max }$ (shown above as $50 \mathrm{mN}$ ), displacements $d_{1}, d_{2}, d_{3}$ are recorded with their corresponding force values $f_{1}, f_{2}, f_{3}$. After mapping the forces to displacements, an exponential function is fitted between the two, which is denoted $A_{1}$.

For the second step of calibration, the $\mathrm{A}_{2}$ force-to-displacement table, a calibration stimulus is indented into the skin to the desired peak force given the parameters from $A_{1}$. Then the stimulus is held for $1.5 \mathrm{~s}$ to determine the magnitude and timing of the force relaxation at that force magnitude (Fig. 5). Force traces of the skin's relaxation starting at the peak force are fit with a triple decay exponential function (Eqn. 6, Fig. 4).

Skin Relaxation $(\mathrm{mN})=\mathrm{Ae}^{-\mathrm{B}\left(\mathrm{t}-\mathrm{t}_{0}\right)}+\mathrm{Ce}^{-\mathrm{D}\left(\mathrm{t}-\mathrm{t}_{0}\right)}+\mathrm{Fe} \mathrm{e}^{-\mathrm{G}\left(\mathrm{t}-\mathrm{t}_{0}\right)}$

Displacement $(m m)=-\ln \left(\left(A e^{-B\left(t-t_{0}\right)}+C e^{-D\left(t-t_{0}\right)}+F e^{-G\left(t-t_{0}\right)}\right) / a\right) / b$

Force fit over the hold of the stimulus by Eqn. 6 can be translated back into displacement using $A_{1}$ force-to-displacement table, as had been identified in Eqn. 5. These displacements over time are used to create the $A_{2}$ portion of the trajectory (Eqn. 7), calculated in intervals of $1 \mathrm{msec}$. In particular, we take displacement at time $t$ and subtract the displacement at the previous millisecond for a total of 1500 movements over the $1.5 \mathrm{~s}$ time period. The velocity of each 
displacement is the derivative of Eqn. 3 at each time t. This velocity determines the smoothness of movement between each point as a result of the acceleration to achieve each velocity during each time interval. Note that between each successive ramp-and-hold indentation is a sleep period of $60 \mathrm{~s}$ to allow the skin to relax completely to its original state.

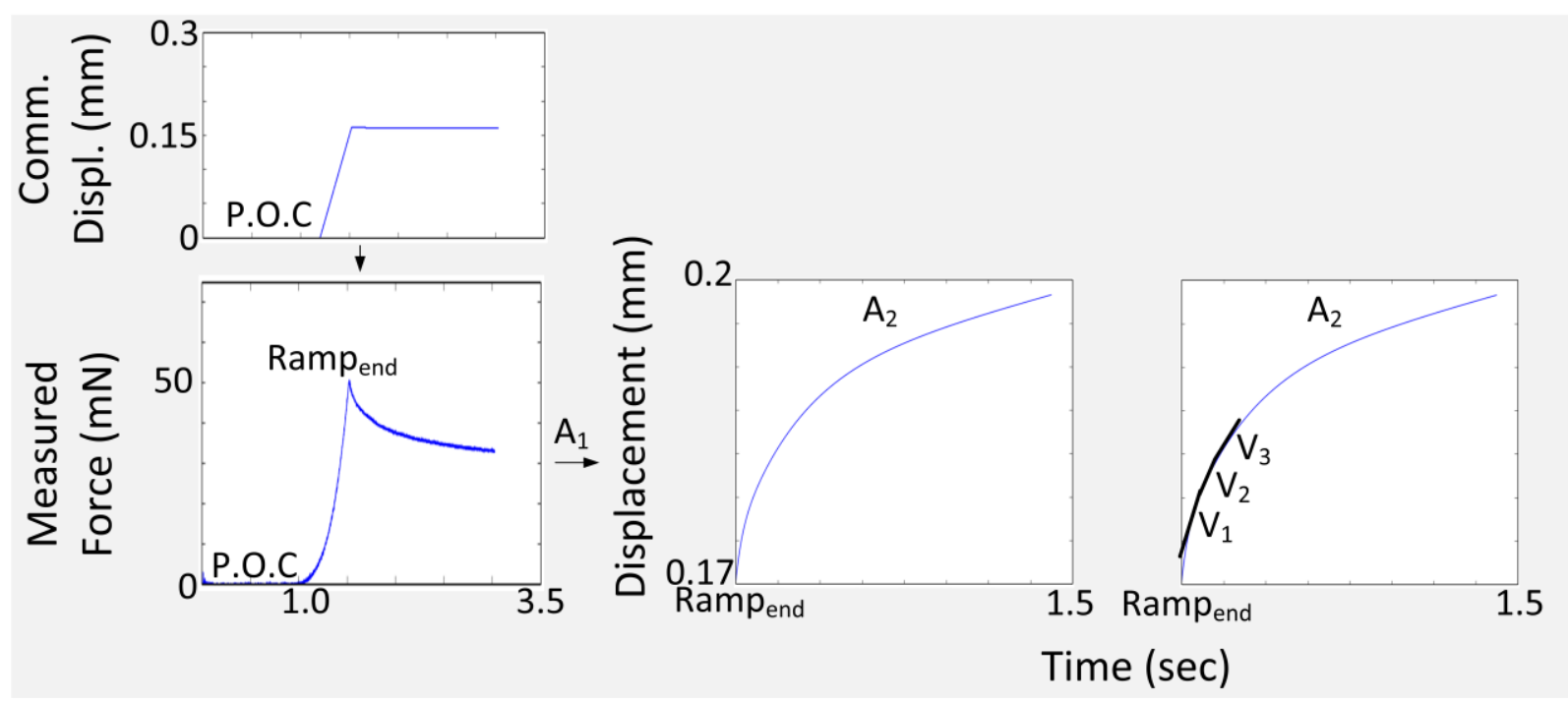

Figure 5: To build up the $A_{2}$ force-to-displacement table that accounts for movements to counteract skin relaxation over the stimulus hold, a calibration stimulus is indented into the skin to the desired peak force (Ramp $_{\text {end }}$ given the parameters from $A_{1}$. Then the stimulus is held for 1-5 seconds to determine the magnitude and timing of the force relaxation at that force magnitude. In the center figure, given the parameters from $A_{1}$, the $A_{2}$ force-to-displacement table is built. In the rightmost figure, from this trace of displacements, velocity is calculated at each millisecond.

\subsubsection{User Controls and Displays}

The system consists of physical controls, software controls and data displays that give the user the ability to manually adjust the $\mathrm{z}$ axis for probe-to-skin contact, manipulate system variables, position the actuator in the $\mathrm{x}$ and $\mathrm{y}$ axis, command control algorithms and collect data. A custom built, three position joystick (Fig. A-3) gives the user the ability to manually actuate the z-stage up, down or idle it at a commanded velocity once activated in the user interface. A potentiometer, protruding from the electronics box, manipulates the operating range of the force 
output from the load cell to increase or decrease force clarity. A separate joystick positions the entire mount system via $\mathrm{x}-\mathrm{y}$ axis stages (Siskiyou, Inc.). The user sends commands to control algorithms (manual, force, and displacement control) through a custom built graphical user interface (Python).

\subsubsection{Graphical User Interface}

The graphical user interface walks the user through the indentation protocol for displacement and force control indentations. The interface splits into two panels: the left panel leads the user through the 5 or 6 steps while the right panel provides a log history, prior indentation status, and continuous feedback from the load cell (Fig. 6). 


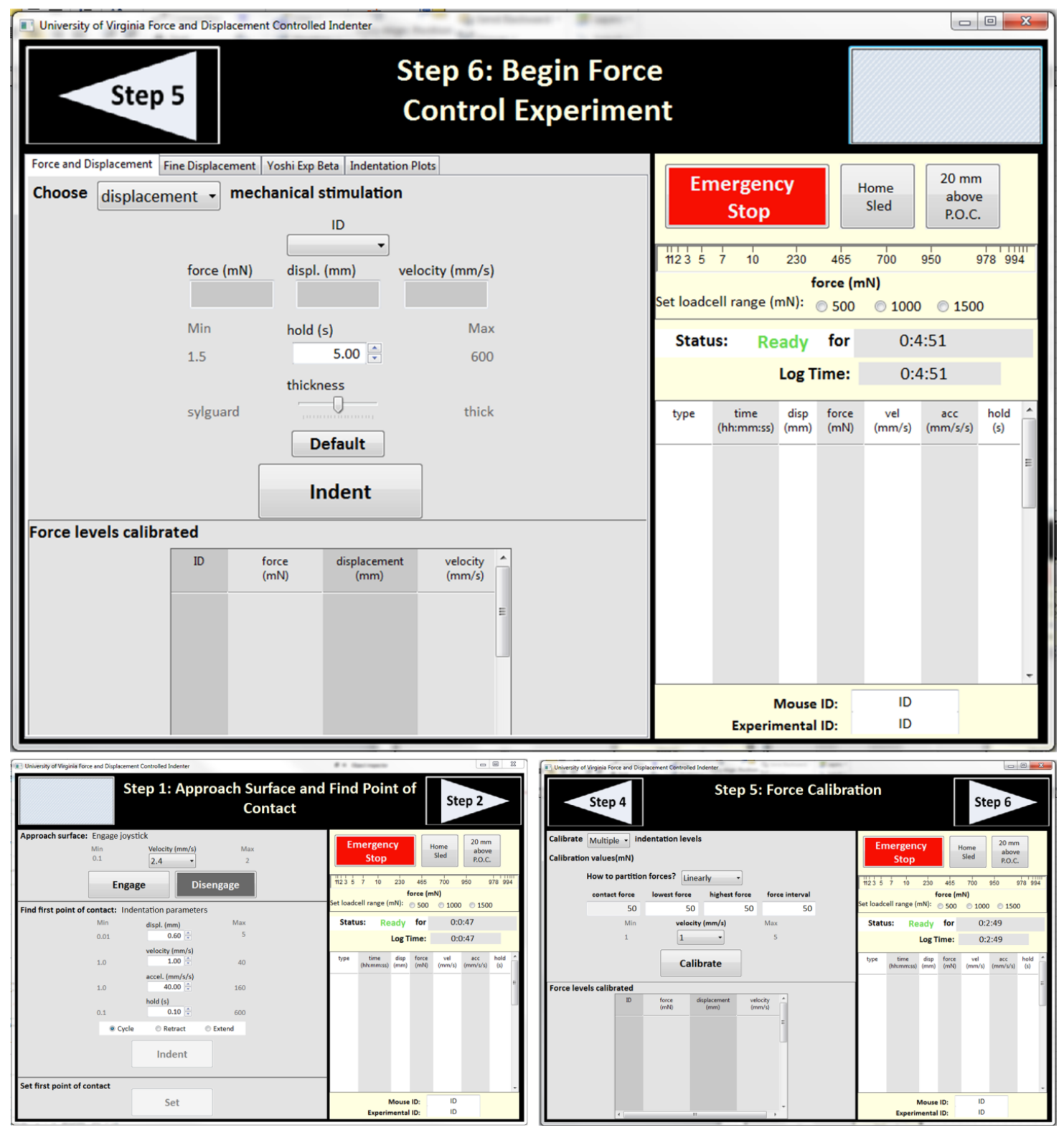

Figure 6: To afford experiments in modes of both force and displacement control, the graphical user interface is based on a series of sequential steps. Each step has an overall goal. For example, step 1 allows the user to position the tip at the surface of the skin and define the first point of contact (bottom left). Before conducting a force controlled stimulation (top), the user chooses a range of forces over which to calibrate (bottom right). Each stimulation is logged in the panel to the right of each screenshot.

Steps 1 (Fig. 6, lower left) through 3 (not shown) allow the user to define the point of contact with the skin and set the lower threshold and saturation forces for an afferent. Step 4 allows the user to choose between experiments in force or displacement control and depending on that choice, step 5 runs displacement control and steps 5 and 6 calibrate and run force control 
(Fig. 6, center and lower right). To validate the design of the interface, the interface went through heuristic evaluations and numerous end-user reviews.

\subsubsection{Data Recording}

Recording software (LabCharts, AD Instruments), located on the same computer as the user interface, connects to a standalone A/D converter (PowerLab Systems 16/35, AD Instruments) through a data translation board (DT3016) and records force (mV), laser displacement $(\mathrm{mV})$ and neural potential $(\mathrm{mV})$ data. The software samples data at $20,000 \mathrm{~Hz}$, displays data to the user, and saves data as a Matlab file for later post-experimental analysis.

\subsubsection{Integration and Electronics Interfacing}

Integrators and electronic interface communicate commands to the sled via the motion controller, power all sensors and the joystick, and digitize feedback and sensor information that is then sent to the control algorithms. Integration and electronics interfacing splits into three components: the motion controller, a custom built electronics box (Fig. A-1,2), and an A/D converter. The motion controller, the XPS model Q8 (Newport Corporation, USA) drives the vertically oriented sled. This motion controller, however, only controls displacement, its rate and acceleration. Therefore, algorithms have been built to modify its function (as described in the section, Control Algorithms). Sensor input for the control algorithms (load cell, laser displacement sensor and operator's manual joystick feedback) pass through a custom built electronics box. The custom built circuitry box powers each sensor and joystick using a global switching power supply (GPM55BG, Condor) and transmits the joystick and displacement laser voltage while amplifying the load cell at a rate dictated by the potentiometer resistance, splitting into the motion controller 
and A/D converter (Powerlab Systems 16/35, AD Instruments), and filtering at a rate of 500 and 5,000 Hz.

\subsection{Experimental Methods}

To test the indenter's performance in both displacement and force control, two experiments were employed: 1) multiple indentations of different stimulus magnitudes into skin tissue of varying thickness and 2) indentation in an ex vivo neural recording from a slowly adapting fiber in the skin of one mouse.

Experiment 1 tested the mechanical indenter's ability to hold different forces in different skin thickness. The indentations consisted of five force levels $(10,20,50,100$ and $200 \mathrm{mN})$ at velocity of $0.5 \mathrm{~mm} / \mathrm{sec}$ with an acceleration of $80 \mathrm{~mm} / \mathrm{sec}^{2}$ by three iterations each and their corresponding displacements were applied in a random order. This added up to a total of 60 indentations. The indentations were delivered to the ex vivo skin of two, 10 week old animals, in three different locations each, leg, belly and back. These skin locations exhibited visually different thickness. The skin was draped on top of a petri dish of silicone-elastomer (Sylgard, Dow Corning) of 0.25 inches thick, neo-hookean hyper-elasticity, with a piece of wet nylon (L'eggs winter-weight pantyhose) between the skin and silicone-elastomer.

In addition to qualitatively evaluating the force clamps individually and their consistency between repetitions, two metrics employed were across the entirety of the hold, the achieved force was subtracted point by point from the commanded force (Eqn. 8) and the percent difference between commanded and achieved force at the first point of the force clamp (Eqn. 9). 
Ave. dev. of $\mathrm{F}_{\text {ach. }}$ from the $\mathrm{F}_{\text {peak }}$ over hold $(\mathrm{mN})=\frac{1}{N} * \sum a b s\left(F_{\text {ach. }}-F_{\text {peak }}\right)$

Percent difference between force ${ }_{\text {achieved }}$ from force comm. $=\frac{\left|\left(F_{\text {ac } \square \text { ieved }}-F_{\text {comm. }}\right)\right|}{F_{c o m}}$

Experiment 2 evaluated the AM afferent's response to force control. One mouse was used of 8 to 10 weeks old. One force level, $300 \mathrm{mN}$, aimed to cover a wide range in the afferent response, and at a velocity of $1 \mathrm{~mm} / \mathrm{sec}$.

All data was analyzed using the Matlab extension of Python. Data for experiment 1 output as a .txt file and contains load cell output from the motion controller's A/D and displacement read from the sled. Data for Experiment 2 were output as .mat files from a separate recording software Lab Charts and contains load cell, displacement laser, and the action potential voltage over time as recorded from the neuron.

\section{Results}

Fig. 7 shows a series of displacement and force controlled indentations from Experiment 1. Visually, the force control in the central Fig. approximately hold the achieved force with slight dips after the trajectory (>1.5 seconds), although the achieved force undershoots that commanded. In Fig. 8, upper, achieved forces of 50, 100, and $200 \mathrm{mN}$ holds all stayed below 5\% of the commanded force and the majority of $20 \mathrm{mN}$ was below $5 \%$, over the duration of the hold. Holding $10 \mathrm{mN}$ below $+-5 \%$ is at the range of the load cells clarity. In Fig. 8, lower, as force increased the deviation of the achieved from the commanded force improved (Fig. 8, bottom). 

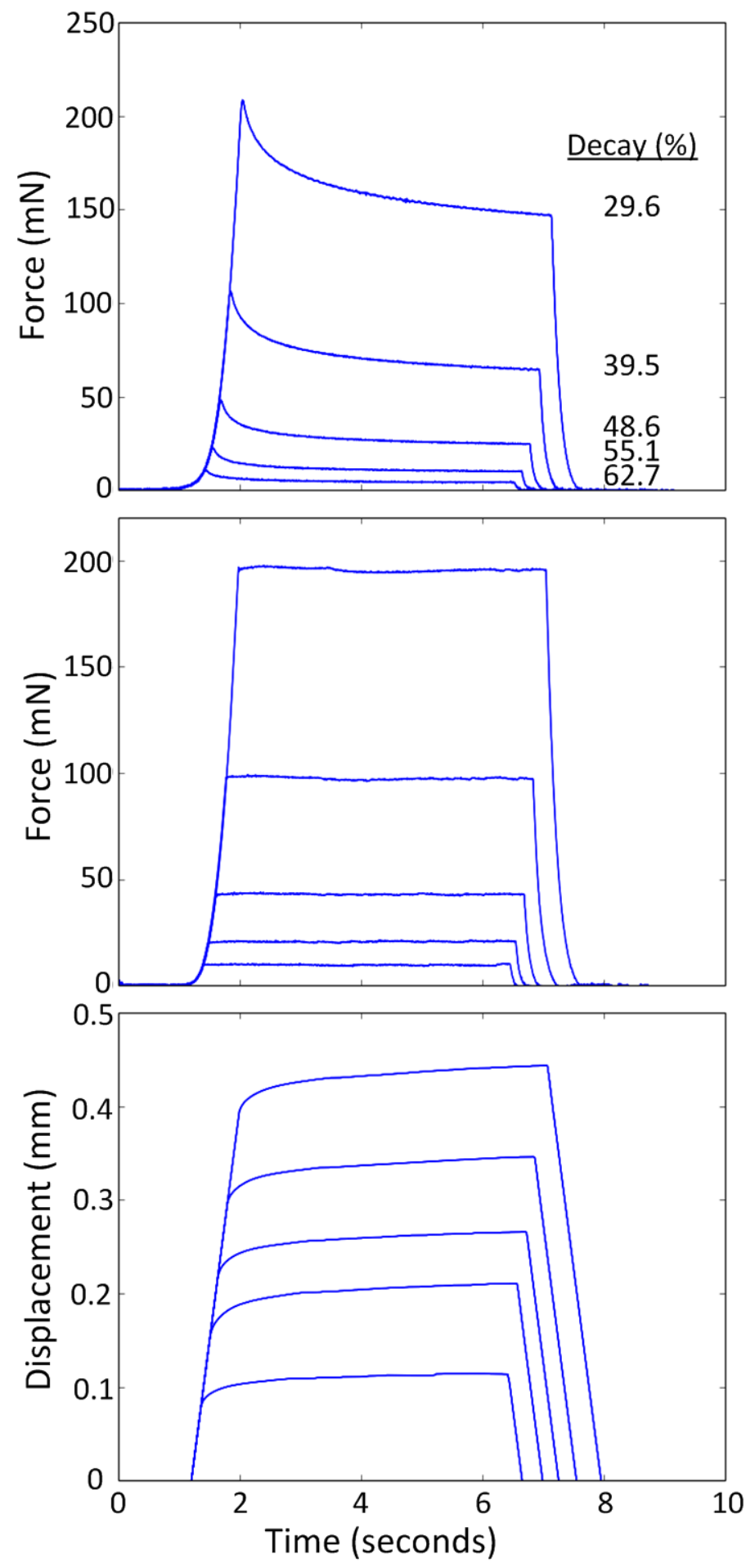

Figure 7: In the upper figure are a set of displacement controlled indentations, where the displacements commanded coincided with peak forces of about 10, 20, 50, 100 and $200 \mathrm{mN}$. Force decays from peak to steady state of between $63 \%$ when peak force is $10 \mathrm{mN}$. At a peak force of $200 \mathrm{mN}$ the force decreases by $30 \%$. In the lower two figures are a set of force controlled indentations, with the force clamp shown in the middle figure and the increasing displacement of the tip needed to maintain that force clamp in the lower figure. 

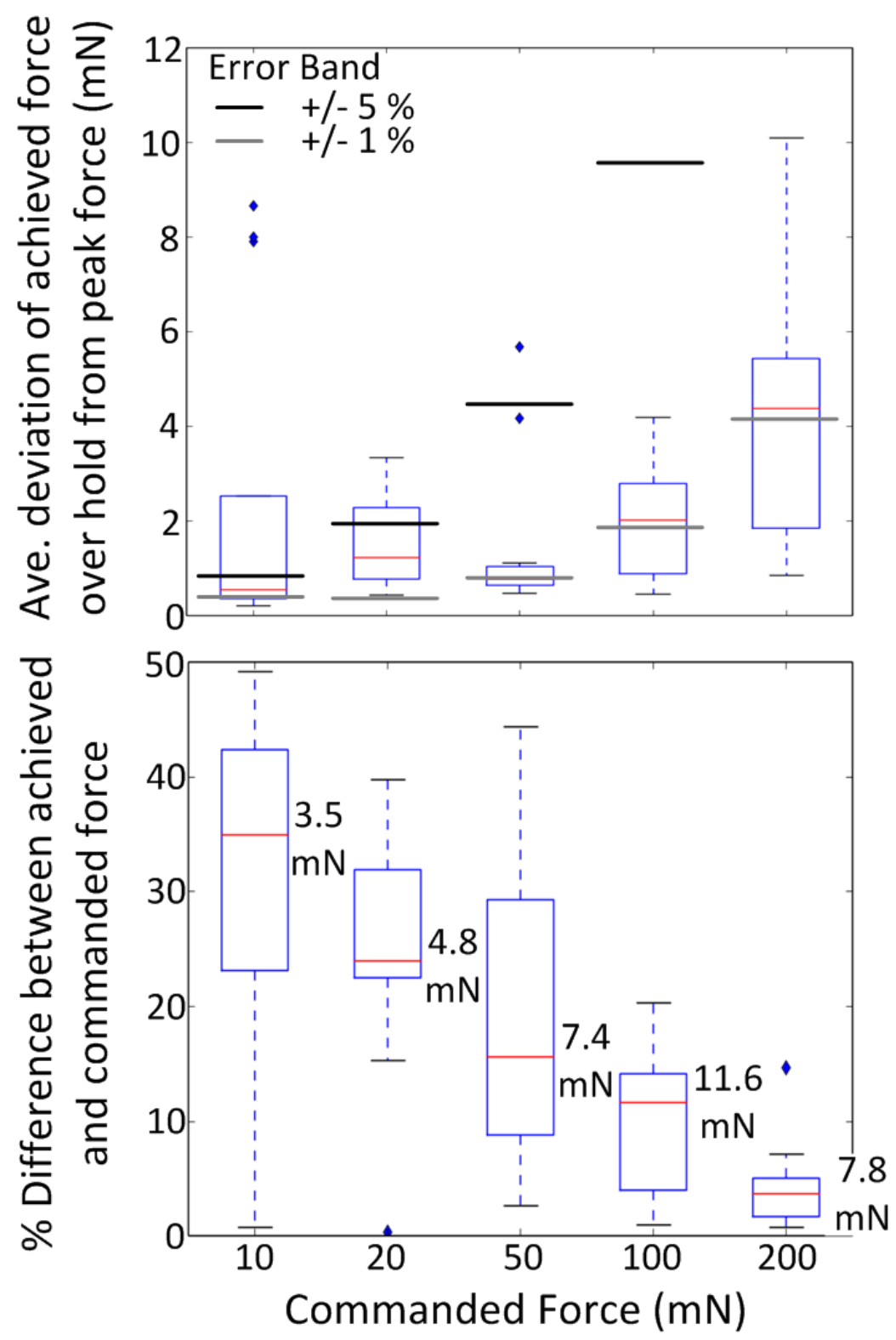

Figure 8: In the top figure, the achieved force was subtracted point by point from the commanded force across the entirety of the hold. It has a positive trend with an increase in force, as well as increase in spread. However, achieved force levels at 10,20 , and $50 \mathrm{mN}$ all have medians of less than $+/-5 \%$ error bar from that commanded. In the lower figure, in force control mode the percent difference between commanded and achieved force has a negative trend with increasing force. Both the maximum and minimum, including outliers, are labeled in $\mathbf{m N}$. In both figures there are 12 iterations per force level across 2 different mice and 3 different body sites per mouse. 
Fig. 9 shows three successive indentations at each of five force levels into the same piece and location on a skin prep. Despite a repeated displacement trace (top), the achieved force for the first indentation is closer to commanded force, where indentation repetitions 2 and 3 fall $3-15$ $\mathrm{mN}$ short of the first indentation. Possible reasoning behind the phenomenon to be further explored in the discussion.
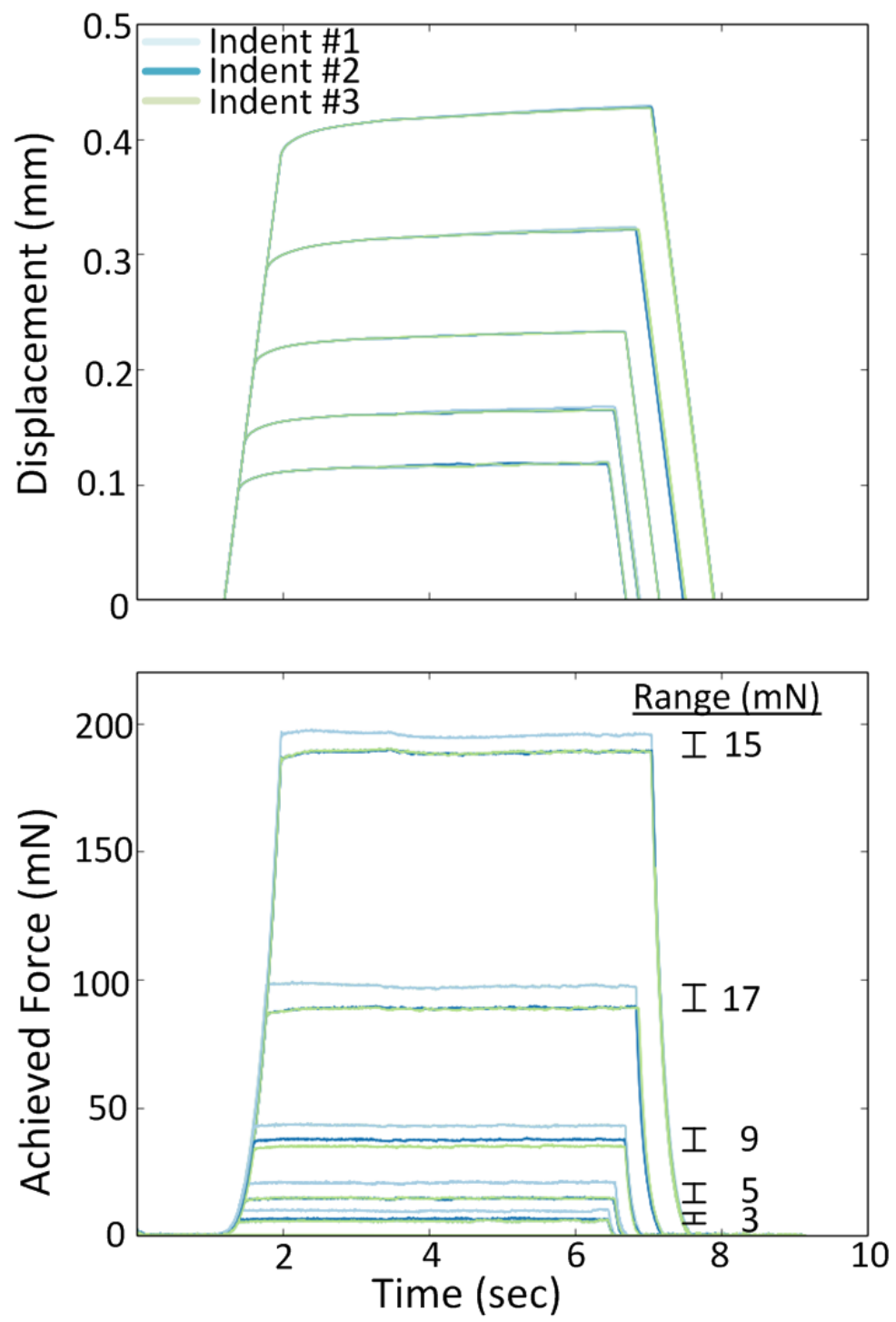

Figure 9: Force control indentations at five levels of commanded force $(10,20,50,100$, and $200 \mathrm{mN})$ with three iterations per level in random order for a total of 15 indentations. While the first set of indentations clamped forces near that commanded, the second and third set of force clamps were noticeably lower than those commanded. The range of achieved force per level of force is listed on the lower image. 
Fig. 10 shows indenter's ability to deliver approximately the same force regardless of skin thickness (Fig. 10).

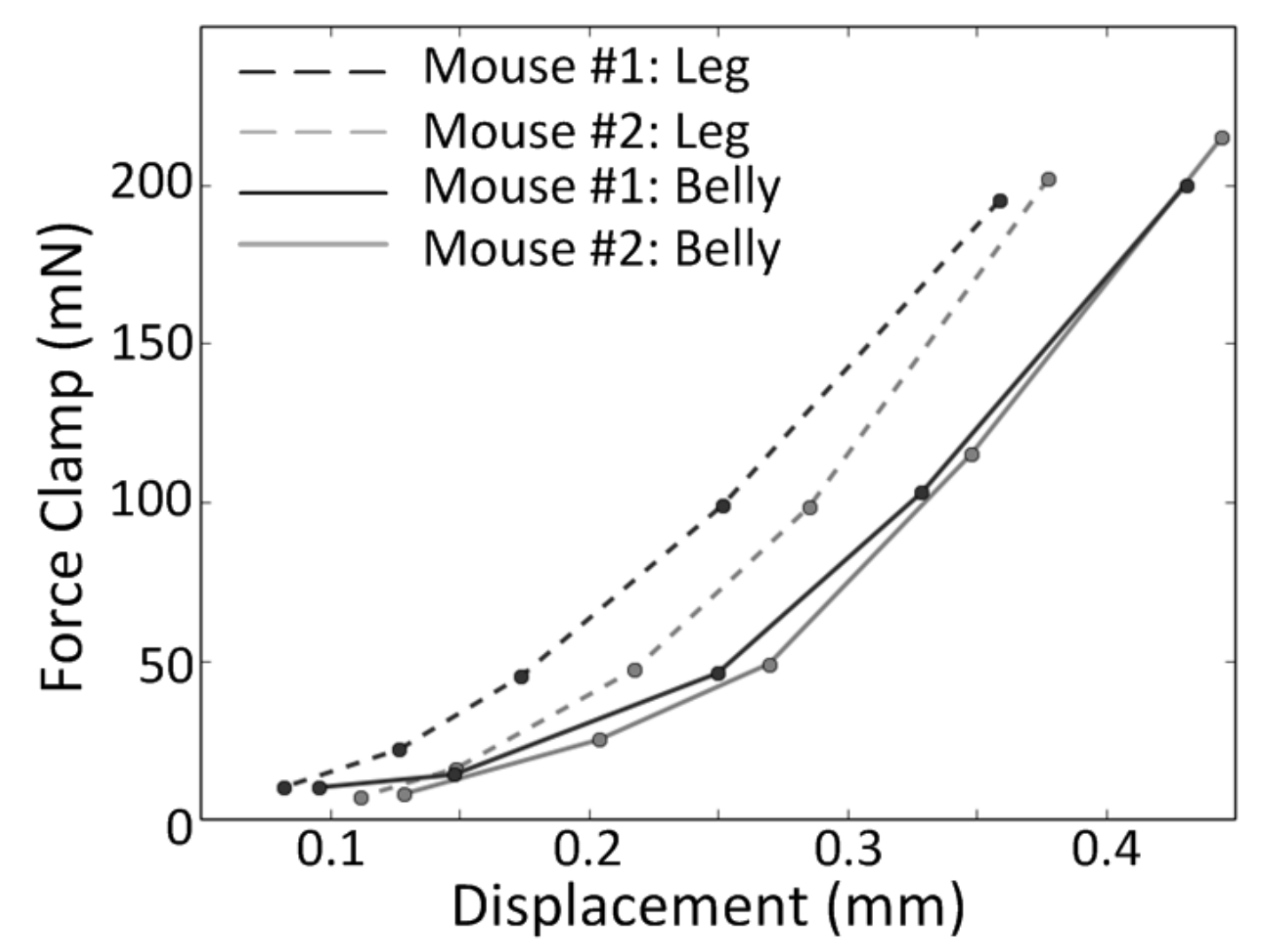

Figure 10: The observed force-displacement relationship differs between animals and body sites. This is likely due to skin thickness, and is in alignment with observations in Wang, et. al (2013). This experiment consisted of 5 different force levels $(10,20,50,100$, and $200 \mathrm{mN})$ across two mice and 2 different body sites for a total of 20 indentations.

Fig. 11 demonstrates the response of a slowly adapting afferent in both force and displacement control. Displacement control at $300 \mathrm{mN}$ elicited a higher differential spike firing, over the early hold of the stimulus as compared to the sustained hold of the stimulus, than force control. The force control indentations maintained firing rate across the entire hold in a much more constant fashion. 


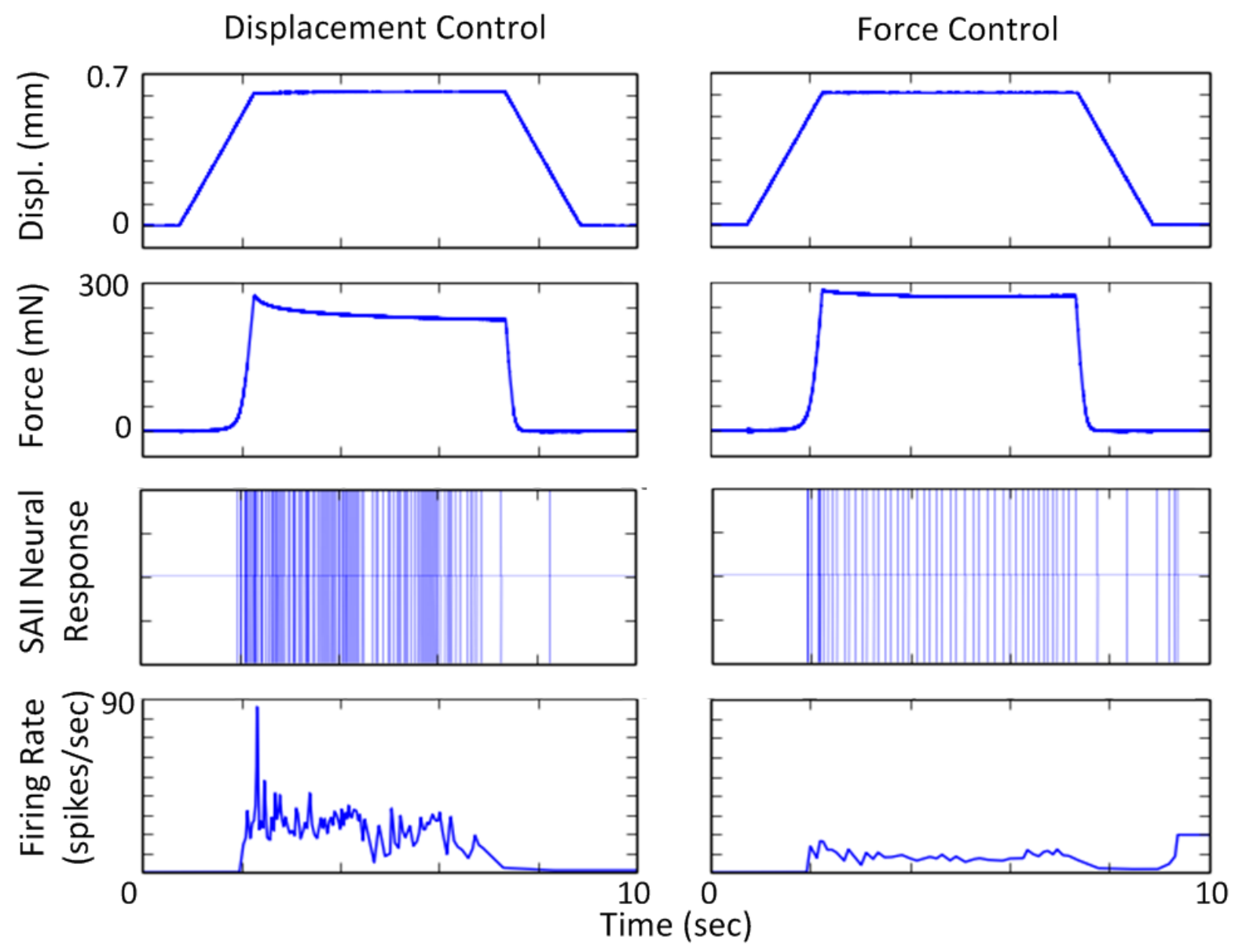

Figure 11: Ex vivo neurophysiological recordings from a mouse $\mathrm{AM}$ afferent in both displacement and force control. In panels A and B, commanded force was about $300 \mathrm{mN}$. The average drop in firing rate from early $(<.5 \mathrm{sec})$ to late hold $(3-5 \mathrm{sec})$ is 30.8 to $17.3 \mathrm{spikes} / \mathrm{sec}(43 \%)$ in displacement control compared to 10.86 and 8.3 spikes/sec $(23 \%)$ in force control.

\section{Discussion}

The work herein describes and demonstrates the design and capabilities of a novel forcecontrolled indenter to operate both force and displacement control, deliver forces over a range of $1-2500 \mathrm{mN}$ with a $1.5 \mathrm{~mm}$ tip, ramp into the skin at about $600 \mathrm{msec}$ to $100 \mathrm{mN}$, and clamping forces to less than 5\% error across the hold. Indenting and clamping force on different parts of the mouse and the afferent readings from experiments 1 and 2 demonstrate the mechanical indenter's ability to hold a clamp within $1-5 \%$ of the achieved force, ramp to and achieve commanded forces for large force values, and indent into, force clamp, and distinguish skins 
with different thicknesses. Studying the neuron revealed the slowly adapting afferent fires as a result of the clamped force and the small displacement adjustments the indenter makes to clamp force don't elicit artificial neural spikes.

Force control indentations are an integral part in studying and understanding neuronal response to stimuli. Force control provides a consistent stimuli to skins of different thicknesses, decouples afferent response from stimuli magnitude and skin relaxation, and it has become more apparent through studies that our body acts and reacts to hold force and not displacement .

The original force control algorithm was entirely PID feedback across the clamp but there were several problems with this approach. Firstly, there was a $50 \mathrm{~ms}$ delay between the ramp period and the start of PID. This delay, during the greatest period of decay, drops the force clamp a large percentage off of the commanded force. Secondly, PID control would require a large number of parameters to account for varying velocity, commanded force, skin thickness, and the difference in decay between the early and late hold. Finally, the controller cycle is 20-30 ms between each movement, again leaving time for decay and large, correctional movements in the early hold.

The force control algorithm herein was built around indentation into a mouse skin on top of sylgard substrate with the use of a $1.5 \mathrm{~mm}$ ceramic tip; however the mechanical indenter can be manipulated to indent into any substrate, skin and with any tip size. To do so, a study must be run to fit, what is likely, an exponential decay function during displacement control, as described in section 3.1. The results of the study will provide an estimation of the optimal exponential decay fit and the trajectory time constants.

There are a few shortcomings with this force control methodology and stimulator. Firstly, the design of the algorithm and timing between movements required heavily on the design 
elements of the motion controller. For example, at max the motion controller can only move 160 $\frac{\mathrm{mm}}{\mathrm{s}^{2}}$, which can limit the controller's ability to fit a calculated trajectory. Other stimulators have a similar problem as there is no ideal motion controller with infinite capabilities. If a trajectory can't be completed as a result of passing the maximum acceleration, the interface gives the user opportunity to re-calibrate at a different velocity. Another artifact and shortcoming of the controller is the discrete nature of trajectory movements. Discrete movements can elicit false afferent firings. Large discrete movements occur in other controllers, notably the Aurora 300 series, as a result of large time intervals between movements (Jang et. al, 2009 and Ge and Khalsa, 2002). The time between movements have been set to $1 \mathrm{msec}$ to limit the size of discrete movements and limit neuronal impact. Finally, experiment one and the metric, \% early hold error, demonstrated the controller's inability to repeat force control indentations at the same force (Fig. 9). A possible reason is that the skin hasn't been pre-conditioned enough and once pre-conditioned, calibrations would be able to repeatedly achieve commanded force control. Other work has included an inertial component to the force read at the tip (Cheng et al., 2013). To rectify the problem, if an initial force is above or below the commanded force, instead of moving towards that force after the trajectory, the system clamps the achieved force. Such a solution, while not ideal, will prevent excessive movement once the trajectory ends, in order to reach the commanded force. Other possible sources of variance could be the phosphate and SIF solution in chamber the mouse skin rests on, skin slipping from attaching to the tip as well as floating on top of the phosphate solution. 


\section{References}

Aurora Scientific, Canada. (2014). Retrieved from: http://www.aurorascientific.com/product/neuroscienceproducts/300c-i-mechanical-stimulator/

Cheng, X et al. (2013). A mechatronic platform for studies of tactile sensory system on prosthesis hand. Intelligent Robotics and Applications (vol. 81, issue 2). p.500-508. Retrieved from: http://link.springer.com/chapter/10.1007\%2F978-3-642-40852-6_51

Ge, W and Khalsa, P. S. (2002) Encoding of compressive stress during indentation by slowly adapting type I mechanoreceptors in rat hairy skin. J Neurophysiology (vol. 87) p. 1686-1693. Retrieved from: http://jn.physiology.org/content/jn/87/4/1686.full.pdf

Jang, J H. et. al (2009) Nociceptive sensitization by complement C5a and C3a in mouse. Retrieved from: http://www.ncbi.nlm.nih.gov/pubmed/20031321

Johnson, K O. (2001) The roles and functions of cutaneous mechanoreceptors. Current Opinion in Neurobiology (vol. 11, issue 4). p. 455-461. Retrieved from: http://www.sciencedirect.com/science/article/pii/S0959438800002348

Johansson, R. S. (2004) First spikes in ensembles of human tactile afferents code complex spatial fingertip events. Nature Neuroscience (vol. 7) p. 170-177. Retrieved from: http://www.nature.com/neuro/journal/v7/n2/full/nn1177.html

Levy, D and Strassman A. M. (2002) Mechanical response properties of A and C primary afferent neurons innervating the rat intracranial dura. Journal of Neurophysiology (vol. 88, issue 6) p. 3021-3031. Retrieved from: http://jn.physiology.org/content/88/6/3021.long

Pubols, B. H. Jr. and Benkich M.E. (1986). Relations between stimulus force, skin displacement, and discharge characteristics of slowly adapting type one cutaneous mechanoreceptors in glaborous skin of squirrel monkey hand. Somatosensory Research (vol 4, issue 2). p.111-125. Retrieved from: http://www.ncbi.nlm.nih.gov/pubmed/3809832

Srinivasan, M.A. and Lamotte, R.H. (1995). Tactile discrimination of softness. Journal of Neurophysiology (vol. 73, issue 1). p. 88-101. Retrieved from: http://www.ncbi.nlm.nih.gov/pubmed/7714593 
Tiest, W.M. and Kappers, A.M. (2009). Cues for Haptic Perception of Compliance. Haptic, IEEE Transactions (vol. 2, issue 4). p. 189-199. Retrieved from: http://ieeexplore.ieee.org/xpls/abs_all.jsp?arnumber=4906991\&tag=1

Wang, Y. et al. (2013) Hyperelastic material properties of mouse skin under compression. Plos one (vol. 8, issue 6). Retrieved from: http://www.plosone.org/article/info\%3Adoi\%2F10.1371\%2Fjournal.pone.0067439

Wang, Y et. al. (2013) Natural variation in skin thickness argues for mechanical stimulus control by force instead of displacement. Joint Eurohaptics Conf Symp Haptic Interface Virtual Environ Teleoper Syst. p. 645-650. Retrieved from: http://ieeexplore.ieee.org/stamp/stamp.jsp?arnumber=06548484\&tag=1

Wellnitz, S.A., Lesniak, D.L., Gerling, G. J., and Lumpkin, E.A. (2010). The regularity of sustained firing reveals two populations of slowly adapting touch receptors in mouse hairy skin. Journal of Neurophysiology. 103 (6). $3378-3388$.

Williams, A et al. (2010) Skin relaxation predicts neural firing rate adaptation in SAI touch receptors. Conf proc IEEE Eng Biol Soc. p. 6678-6681. Retried from: http://ieeexplore.ieee.org/stamp/stamp.jsp?tp=\&arnumber $=5626264$ 
Appendix A: Circuit diagrams for power supply, electronics box, and manual joystick

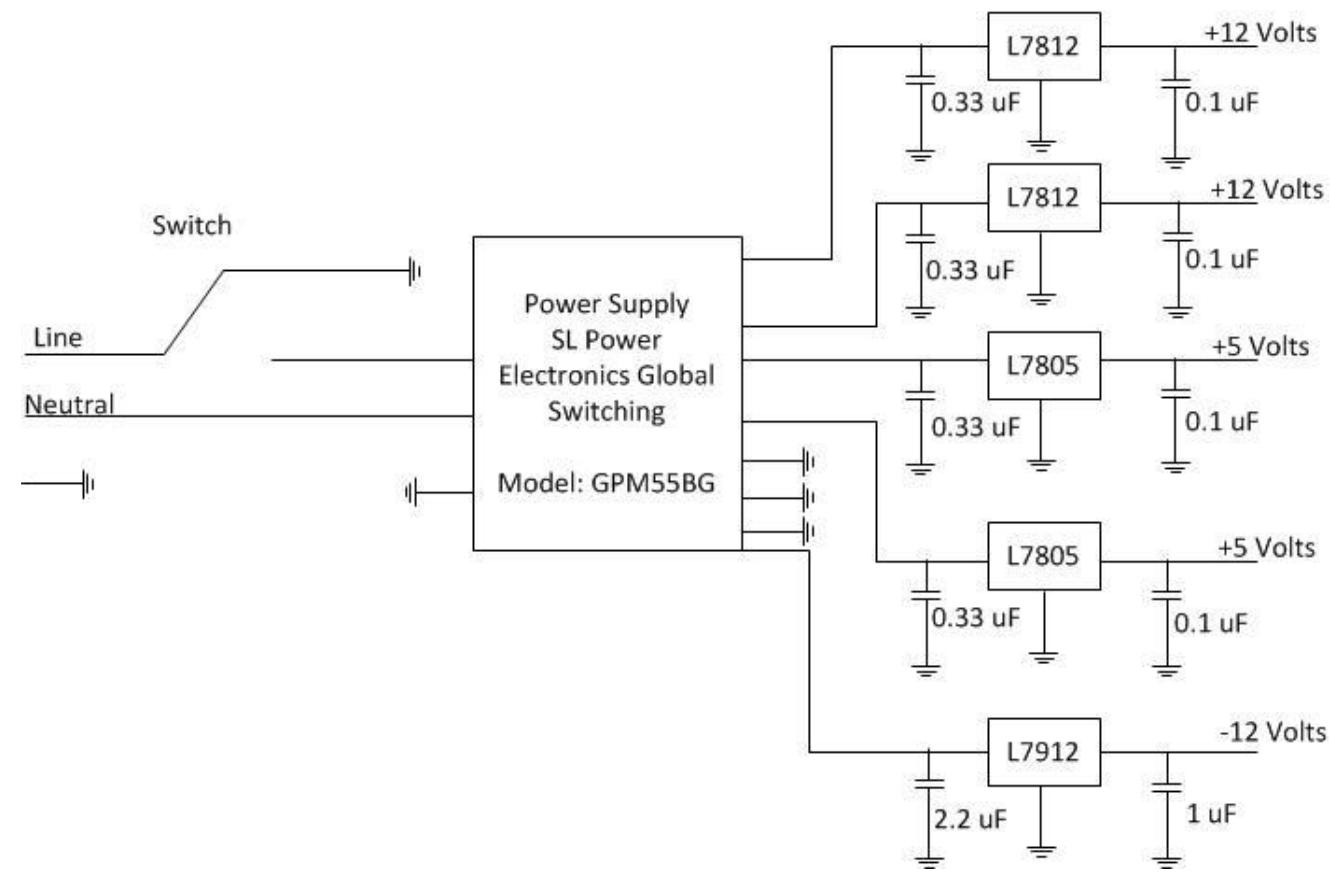

Figure A-1: Power supply and voltage clamps that power the sensors and manual joystick.

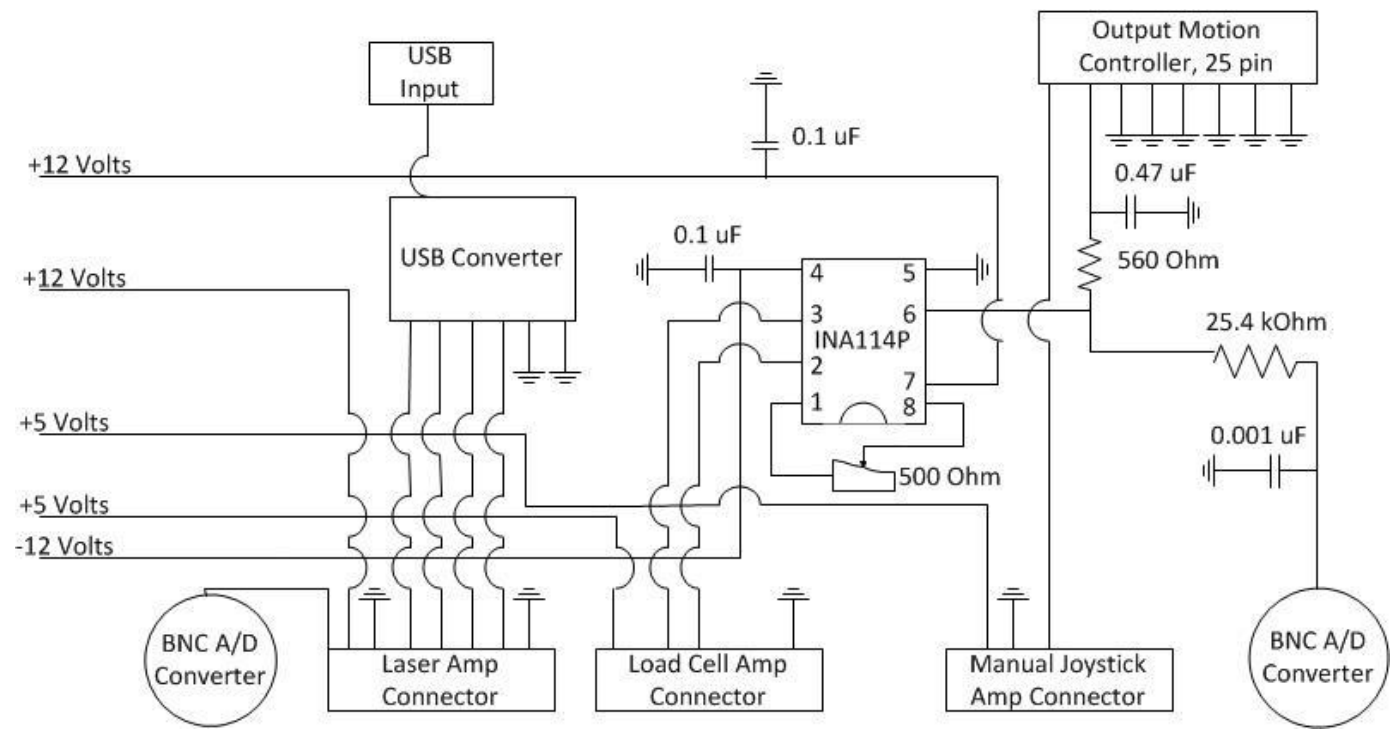

Figure A-2: Connections to the sensors and manual joystick. This part of the power passes signals from the sensors to both of the motion controller and A/D converter. After splitting the load cell signal, the signals pass through low pass filters that filter the signal at 500 and $5000 \mathrm{~Hz}$ and then travel into the motion controller and $\mathbf{A} / \mathrm{D}$ converter, respectively. 


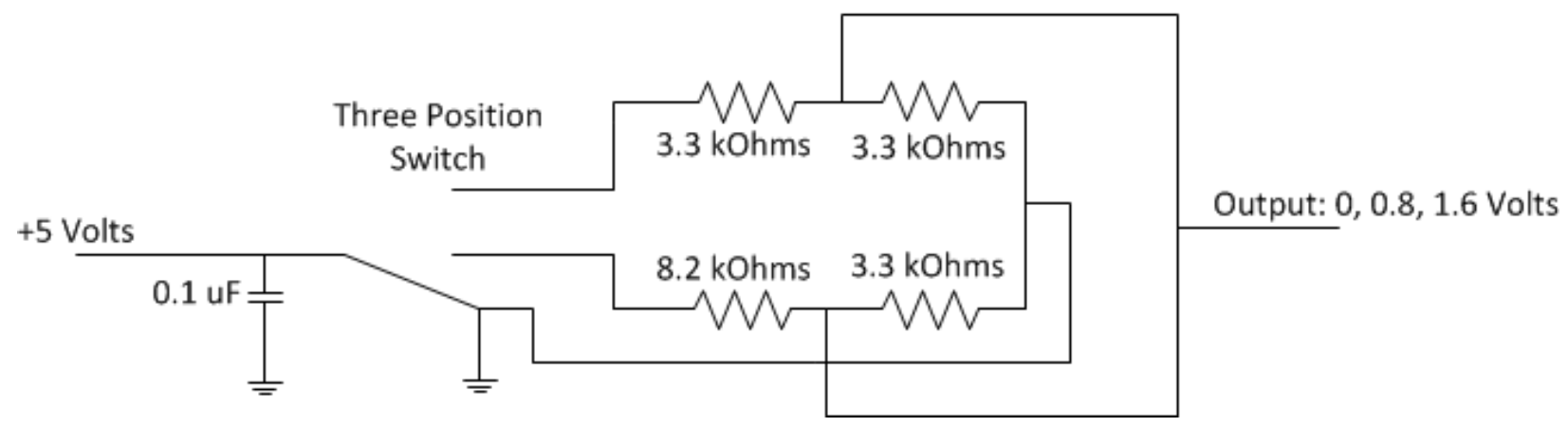

Figure A-3: Manual joystick that responds and sends voltage signals to the motion controller based off of the position of a three position switch.

Appendix B: Specifications on equipment mounts, including the probe tip, t-beam, etc.

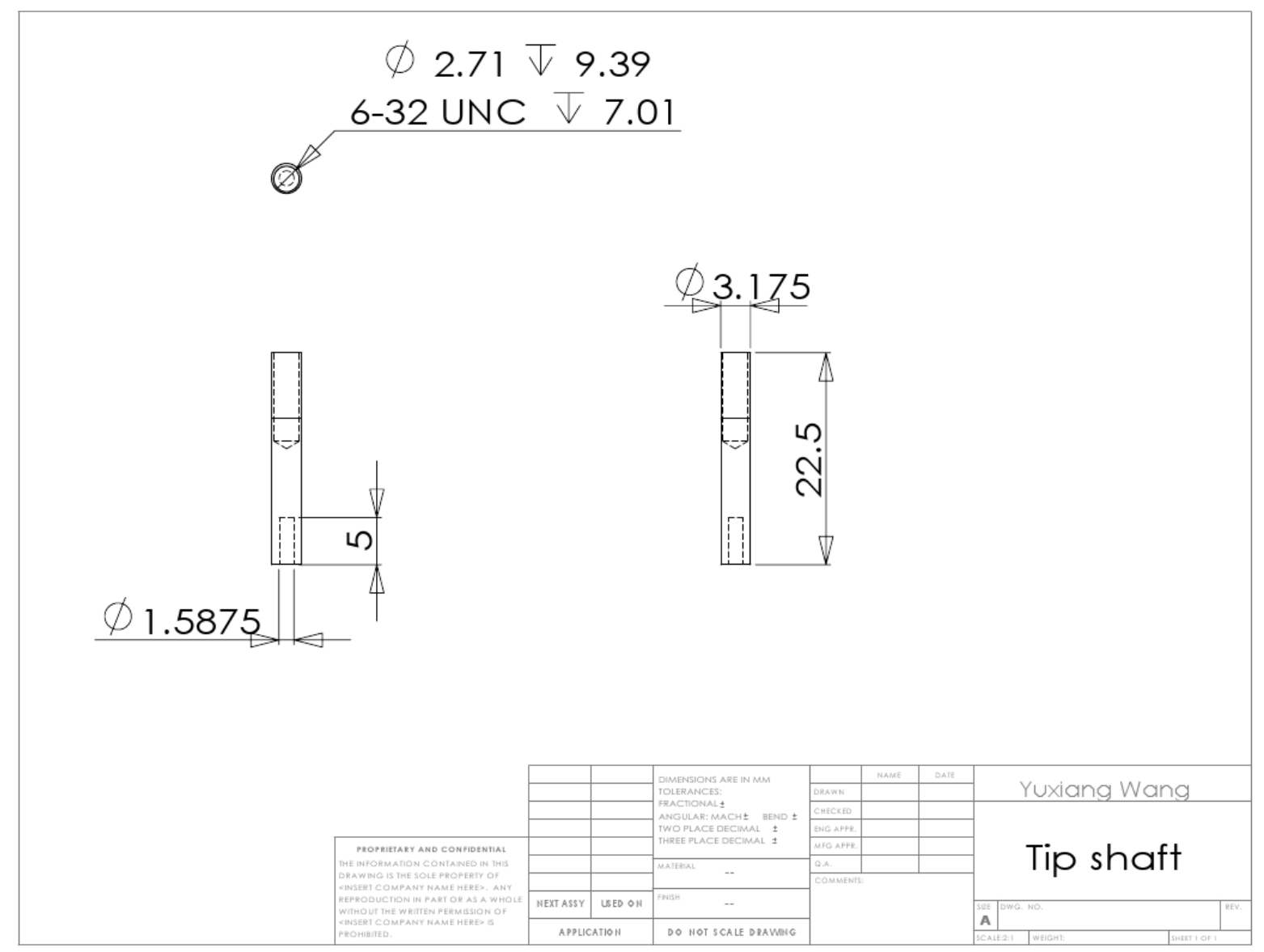

Figure B-1: Tip Shaft 


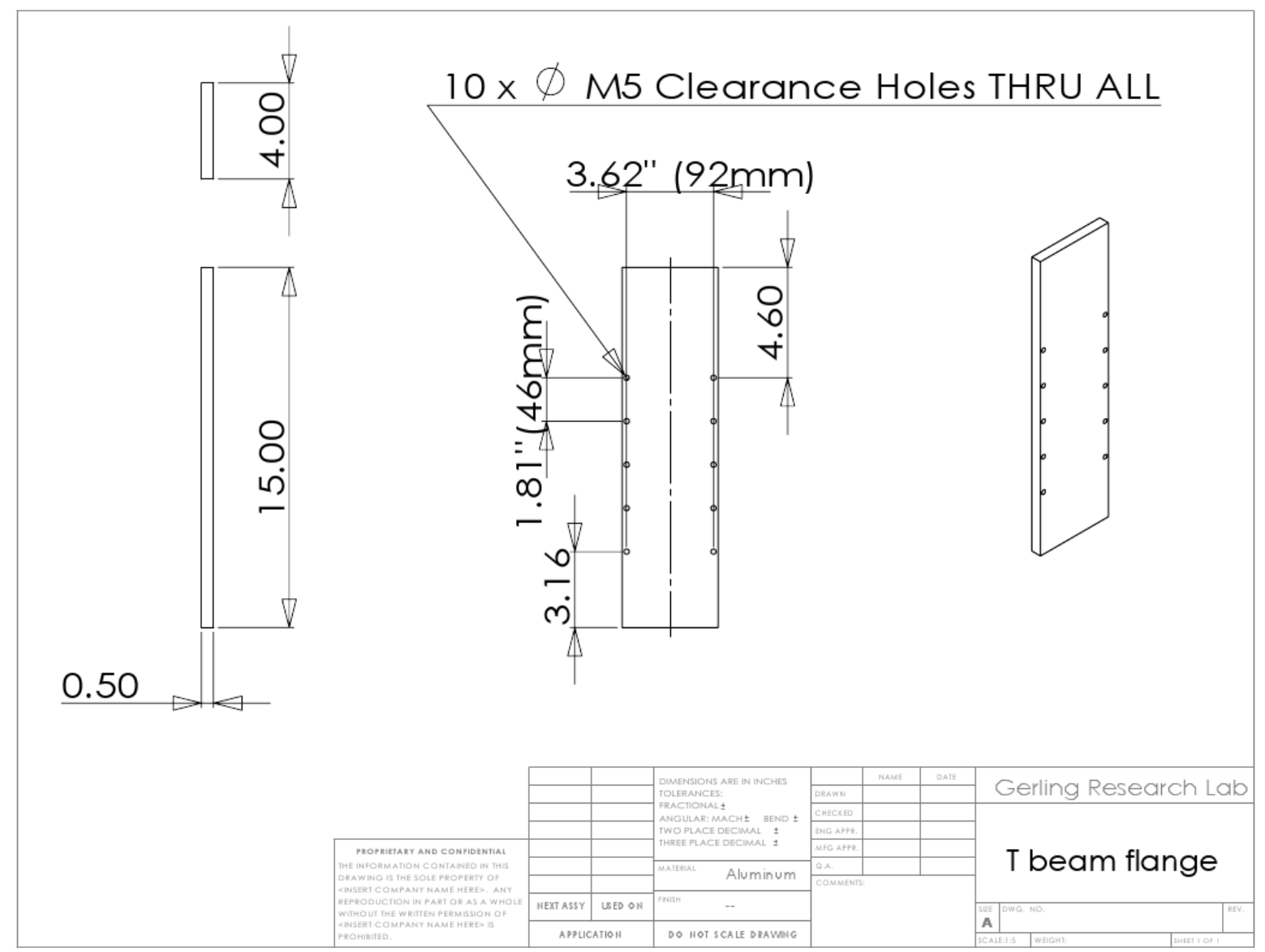

Figure B-2: T-Beam flange 


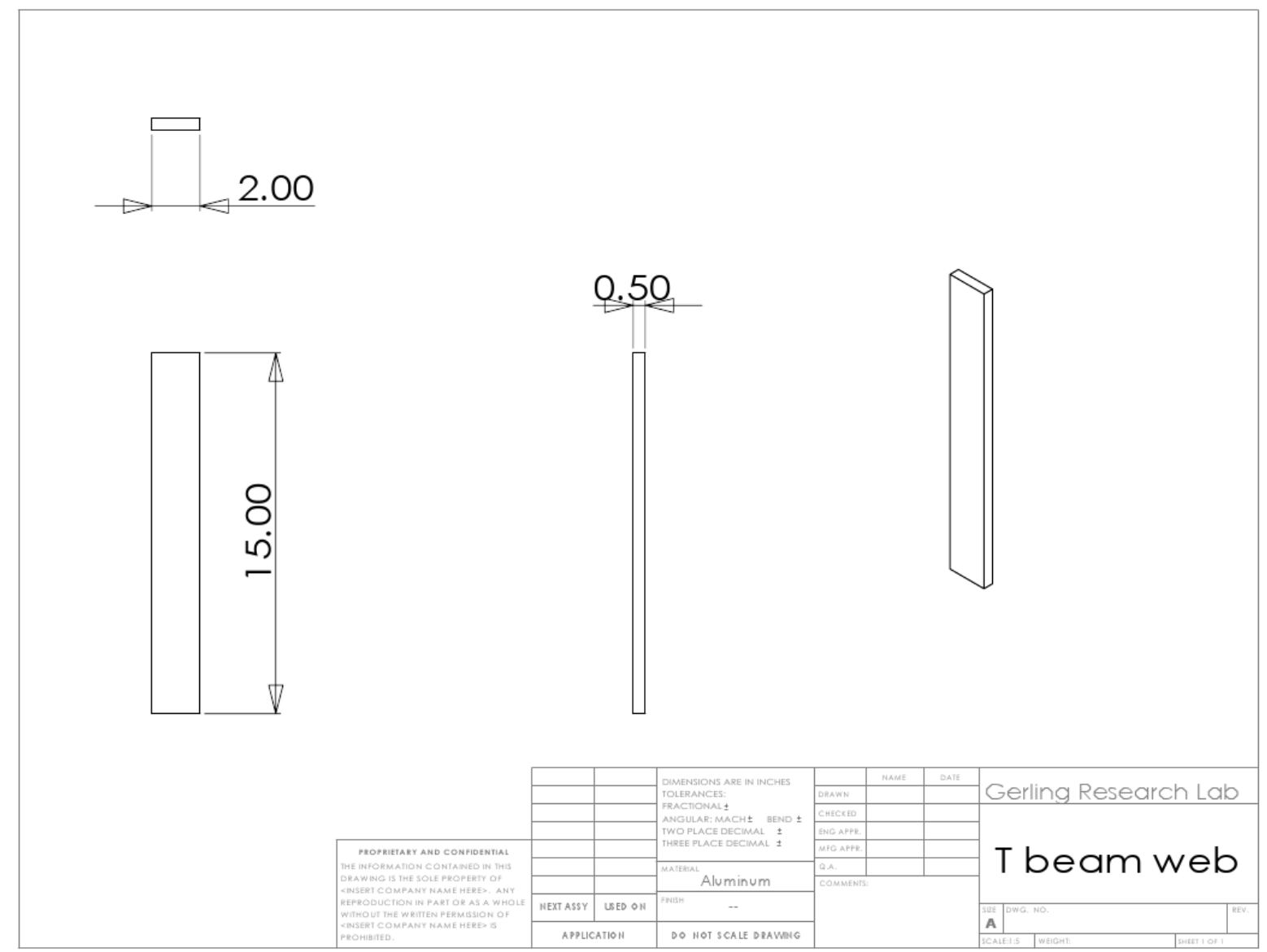

Figure B-3: T-beam web 


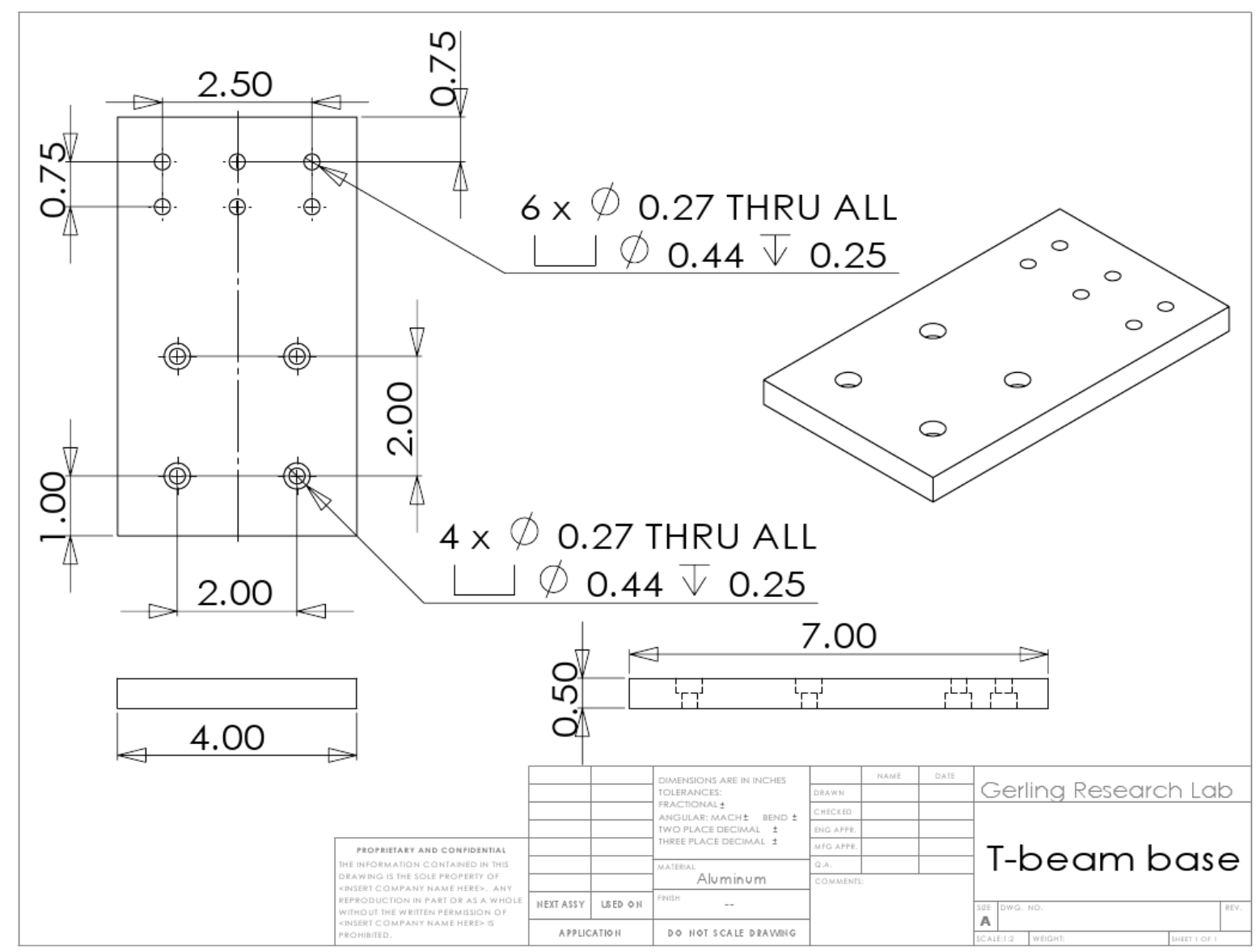

Figure B-4: T-beam base 


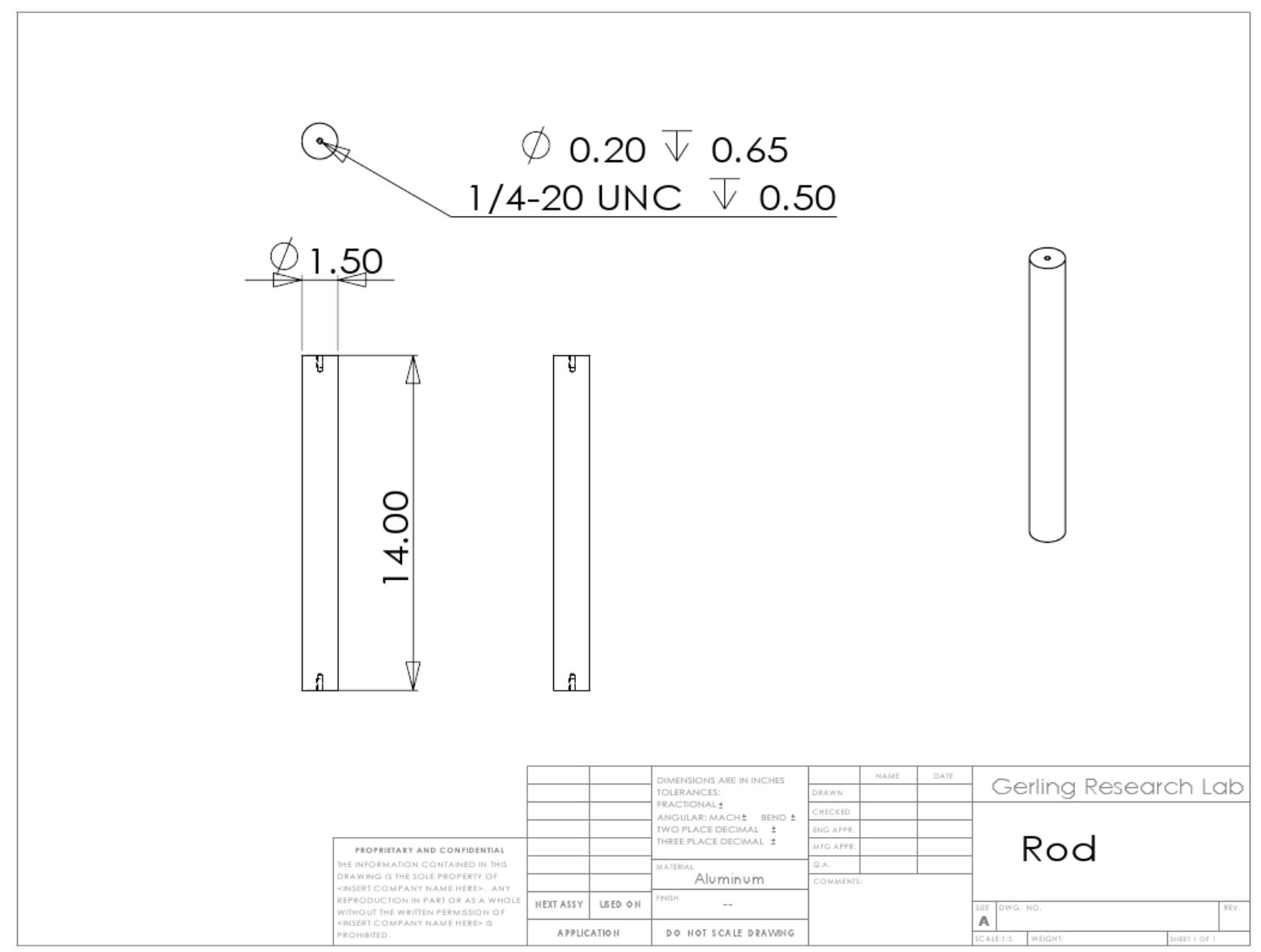

Figure B-5: Rod 


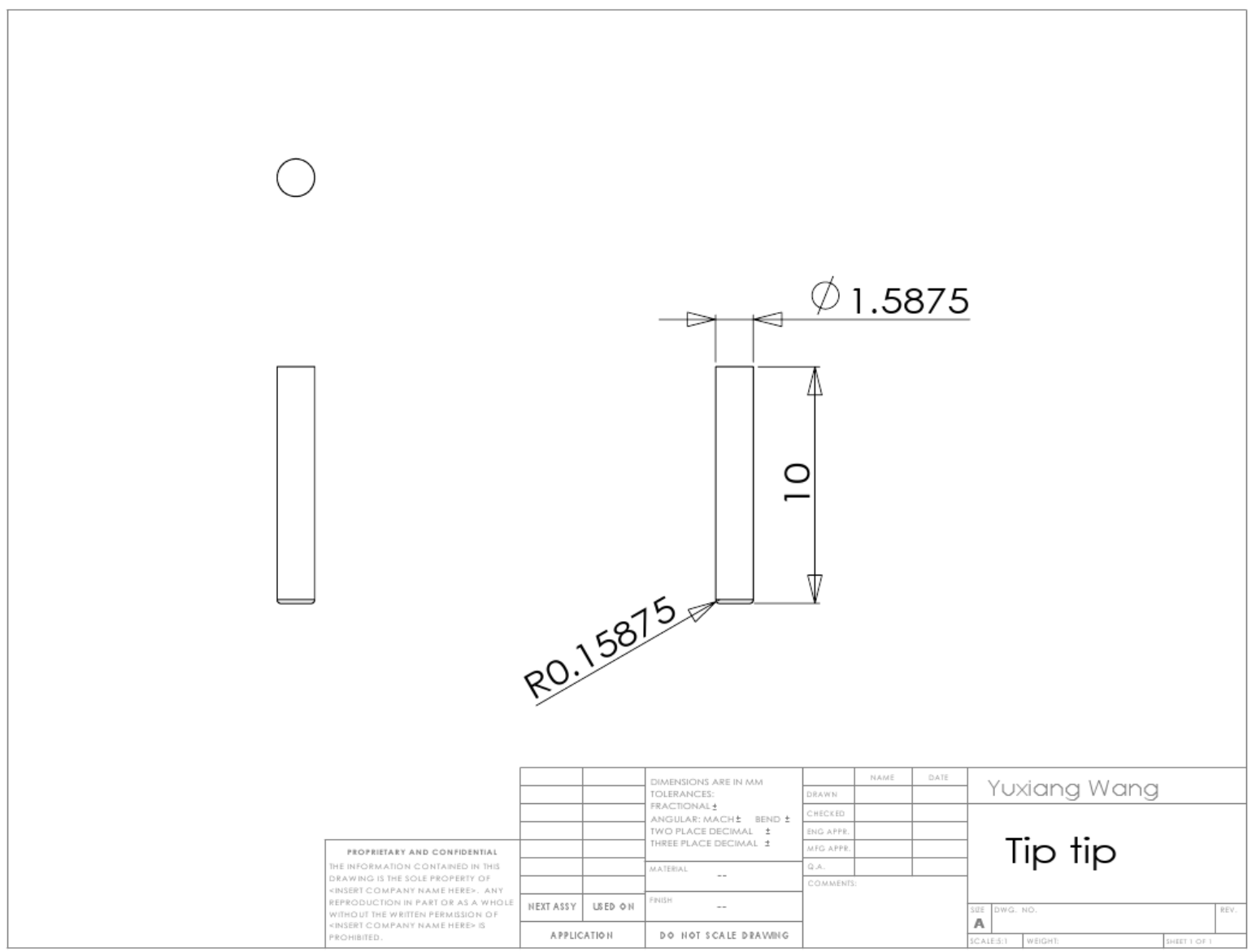

Figure B-6: Tip 


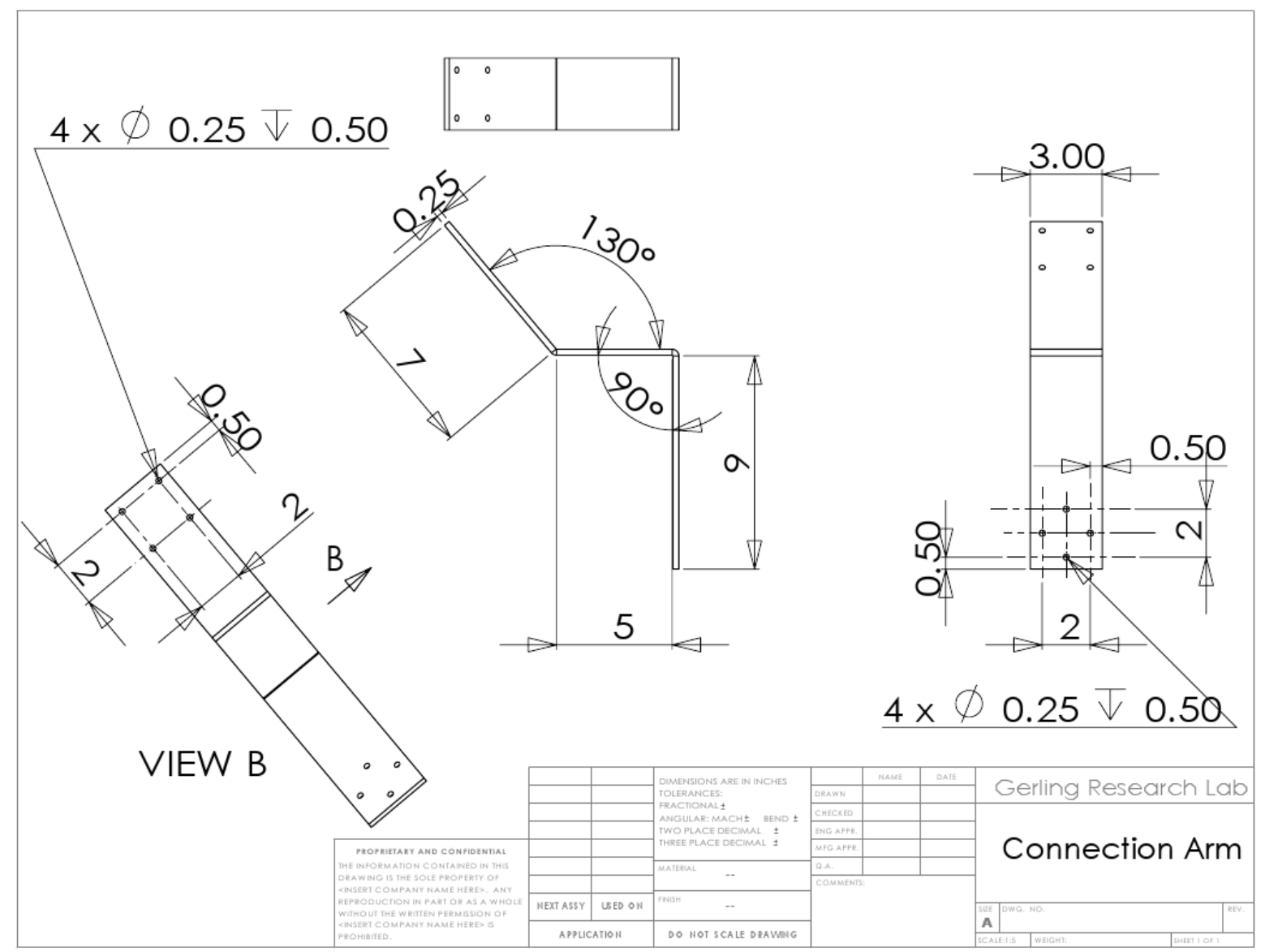

Figure B-7: Connection Arm 


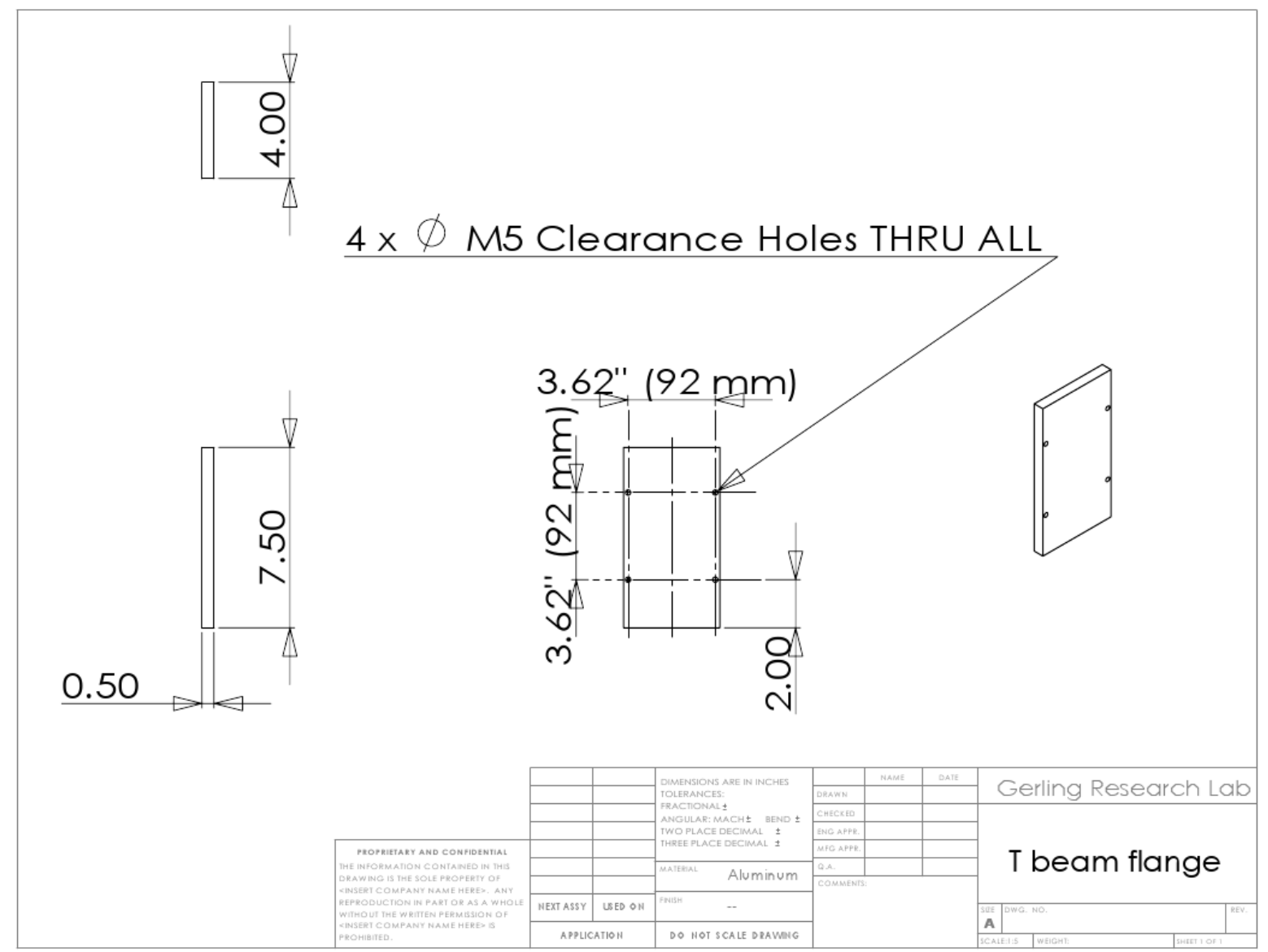

Figure B-8: T-beam flange 


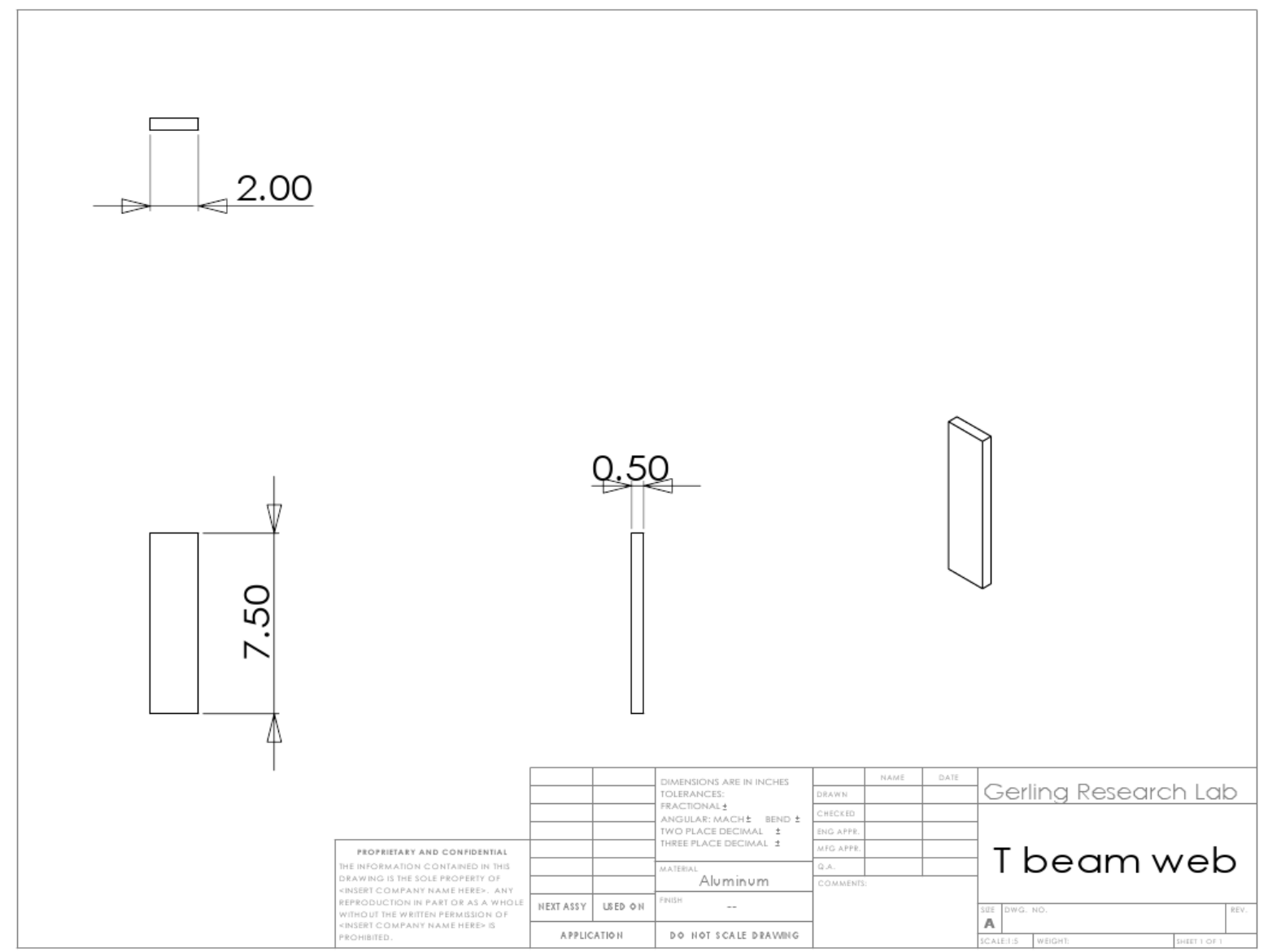

Figure B-9: T-beam web 


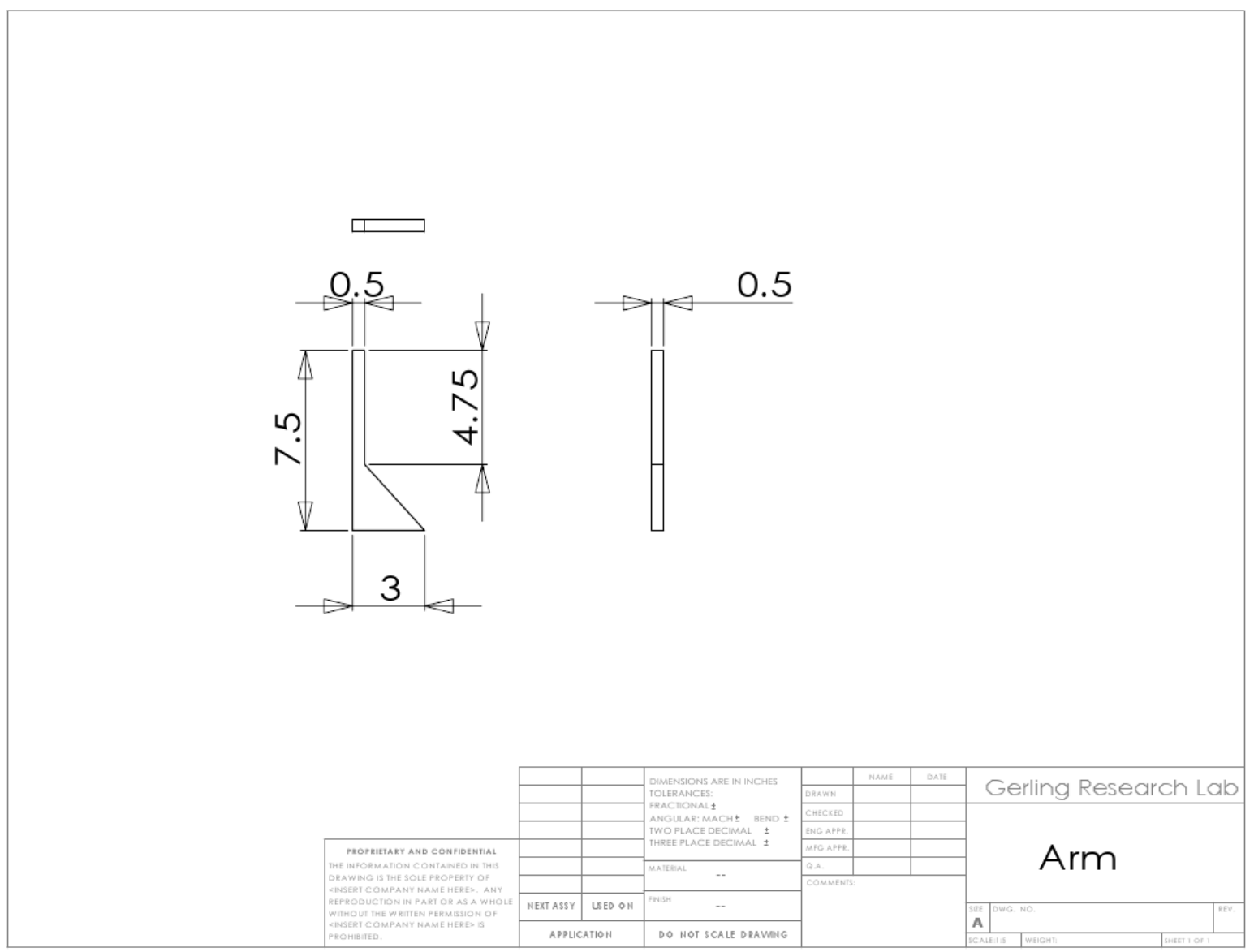

Figure B-9: Arm 


\section{Appendix C- Python code for the user interface}

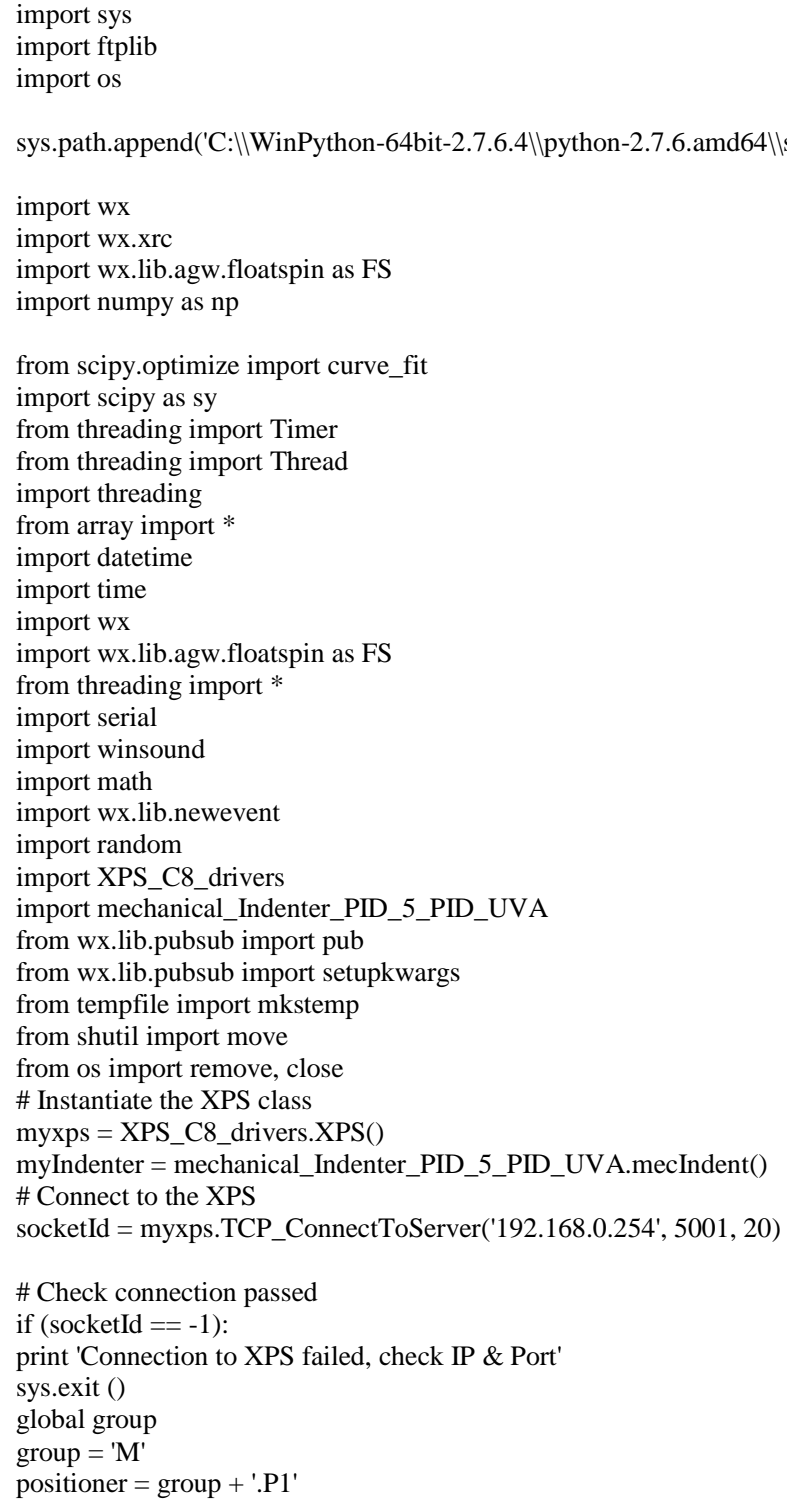


fileLoc = 'C:IWinPython-64bit-2.7.6.4\python-2.7.6.amd64\ColumbiaTest'

servoCycle $=10$

else:

trajectRoute $=$ 'Public/Trajectories'

fileLoc $=$ 'C: $\backslash$ Documents and Settings $\backslash$ Lumpkin LablDesktop $\backslash$ VirginiaForceIndent'

servoCycle $=8$

global yInt

yInt $=0$

global pointOfContact_1

pointOfContact_1 $=0$

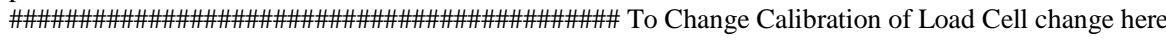

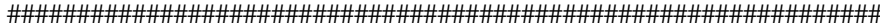

def loadCellCalibrateForce(force):

global yInt

multiplier $=0$

if(univ_Var $==$ 'UVA'):

multiplier $=7.5079$

else:

multiplier $=6.022$

return multiplier*force+yInt

def loadCellCalibrateVoltage(voltage):

global yInt

multiplier $=0$

if(univ_Var == 'UVA'):

multiplier $=7.5079$

else:

multiplier $=6.022$

\#calibrationFunc.setPosition(str(multiplier)+"*x+"+str(yInt))

return (voltage+(-yInt))/multiplier

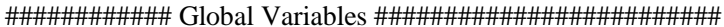

global manMode

manMode $=$ False

global dispMode

dispMode $=$ False

global save_log_dialogFileName

save_log_dialogFileName = "

global checkBox_ID

checkBox_ID $=3000$

global checkBox_ID_f

checkBox_ID_f $=4000$

global checkBox_ID_force

checkBox_ID_force $=5000$

checkBox_ID_disp $=5001$

global boxID_fileName

boxID_fileName $=$ []

global forceID_fileName

forceID_fileName $=$ []

global fineboxID_fileName

fineboxID_fileName $=[]$

global disp_CheckBoxCounter

disp_CheckBoxCounter $=$ []

global cb_list

cb_list $=$ []

global cb_list_f

cb_list_f = []

global cb_list_force

global cb_list_disp

cb_list_force $=$ []

cb_list_disp = []

global compForceCounter

compForceCounter $=0$

global CompleteDisplacement

CompleteDisplacement = []

global feedForward

feedForward = [] 
global CompleteForce

CompleteForce $=[]$

global peakRange

peakRange $=1000$

global cw

$\mathrm{cw}=0$

global Alone

Alone $=$ True

global potent_Mode

global presentImages

presentImages = []

global checkBox_ID_val

checkBox_ID_val $=\overline{5} 000$

global cb_list_Values

cb_list_Values $=$ []

global checkBox_ID_values

checkBox_ID_values = []

global tauVal

tauVal = []

global minMaxForce

minMaxForce $=0$

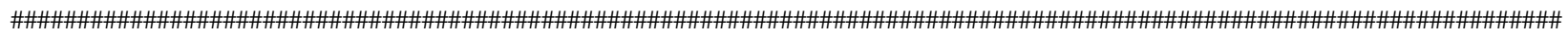
\#\#\#\#\#\#\#\#\#\#\#\#\#\#\#\#\#\#\#\#\#\#\#\#\#\#\#\#\#\#\#\#\#\#\#

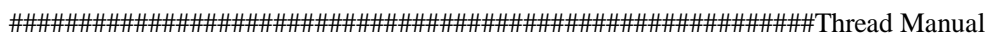

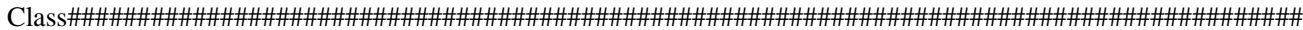

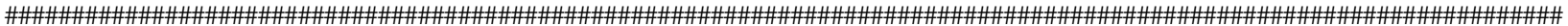
\#\#\#\#\#\#\#\#\#\#\#\#\#\#\#\#\#\#\#\#\#\#\#\#\#\#\#\#\#\#\#\#\#\#

\#\#\#\#\#\#\#\#\#\#\#\#\#\#\#\#\#\#\#\#\#\#\#\#\#\#\#\#\#\#\#\#\#\#\#\#\#\#\#\#\#\#\#\#\#\#\#\#Thread Manual

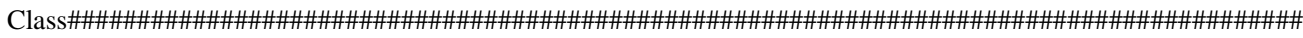

class ManualThread (threading.Thread):

\#pause $=$ threading.Event ()

stop $=$ False

def__init__ (self):

Thread._init_(self)

self.start()

self. stoprequest $=$ threading. Event ()

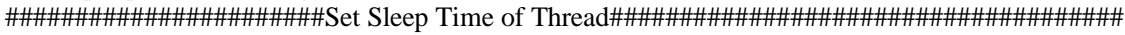

def sleep(self,sec):

time.sleep $(\mathrm{sec})$

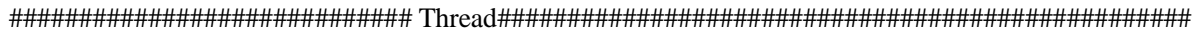

def run(self):

global yInt

global univ_Var

global manual_speed

global upSpeed

global downSpeed

if(univ_Var == 'UVA'):

down_Volt $=3.5$

up_Volt $=4.5$

else:

up_Volt $=1.05$

down_Volt $=.1$

\#\#\#Clear force entries on other pages\#\#\#\#\#\#\#\#\#\#\#\#

\#ForceEntries.Clear()

\#ForceEntries1.Clear()

\#ForceEntries2.Clear()

\#for $\mathrm{m}$ in range(len(CompleteForce)):

\# CompleteForce.pop([m])

\# CompleteDisplacement.pop([m])

up_Volt $=0$

down_Volt $=0$

stop_now $=$ False

count $\mathrm{Y}=0$

volt $Y=0$ 
startTime $=$ datetime.datetime.now ()

stopTime $=$ startTime + datetime. timedelta $($ seconds $=.2$ )

while(not(stop_now)):

timeCurrent $=$ datetime.datetime.now ()

if(timeCurrent>stopTime):

stop_now $=$ True

voltY $=$ voltY+ myxps.GPIOAnalogGet(socketId,["GPIO2.ADC1"])[1]

count $Y=$ count $Y+1$

yInt $=$ float $($ voltY $) /$ count $Y$

\#\#\#\#\#\#Create and add log file entry\#\#\#\#\#\#\#\#

\#time_string = datetime.datetime.now()

\#vel_string $=\operatorname{str}($ speed.getfloats2 $\operatorname{var}())$

\#addManualModeFileEntry(time_string,vel_string)

\#addManualModeLogEntry(time_string,vel_string)

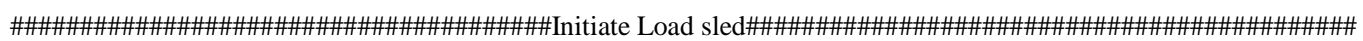

myIndenter.greeting(socketId,group)

\#\#\#\#\#\#\#\#\#\#\#\#\#\#\#\#\#\#\# Retrieve speed from text box, set both sled upward movement and sled downward movement based off of this variable.

upSpeed $=$-manual_speed

downSpeed $=$ manual_speed

\#\#\#\#\#\#\#\#\#Count the amount of times the manual mode has been engaged. Initiate the status of the sled to stopped and

areStopped $=$ True

engaged $=$ False

\#\#\#\#\# Enable group jog mode setting within motion controller\#\#\#\#\#\#\#

[errorCode,returnString]=myxps.GroupJogModeEnable(socketId,group)

if(errorCode !=0):

myIndenter.displayErrorAndClose (socketId, errorCode, 'GroupJogModeEnable')

sys.exit()

\#thresholdCount $=0$

\#firstContact $1=0$

count $=0$

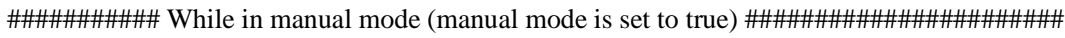

while(manMode):

if $($ count $\% 50==0)$ :

cw_val = loadCellCalibrateVoltage(myxps.GPIOAnalogGet(socketId,["GPIO2.ADC1"])[1])*1000

wx.CallAfter(pub.sendMessage,"cw",cw_update = cw_val)

\#Read Voltage from box

if(GPIOLocation.__eq_("GPIO2.ADC2"))

voltage $2=$ myxps.GPIOAnalogGet(socketId,[GPIOLocation]) $[1]$

if(voltage $2>3.5$ and voltage $2<4.5)$ :

$\#$ \#if(voltage $2>.1$ and voltage $2<1.05)$ :

areStopped $=$ False

[errorCode, returnString] = myxps.GroupJogParametersSet(socketId, group,[upSpeed],[10])

if (errorCode != 0):

myIndenter.displayErrorAndClose (socketId, errorCode, 'GroupJogParametersSet Up')

sys.exit()

elif(voltage $2<3.5)$ :

\#elif(voltage2>1.1):

areStopped $=$ False

[errorCode, returnString] = myxps.GroupJogParametersSet(socketId, group, [downSpeed],[10])

if (errorCode $!=0$ ):

myIndenter.displayErrorAndClose (socketId, errorCode, 'GroupJogParametersSet Down')

sys.exit()

elif(voltage $2>4.5)$ :

\#elif(voltage2<.1):

areStopped $=$ True

[errorCode,returnString]=myxps.GroupMoveAbort(socketId,group)

if(errorCode !=0):

myIndenter.displayErrorAndClose (socketId, errorCode, 'GroupMoveAbort')

sys.exit()

[errorCode,returnString]=myxps.GroupJogModeEnable(socketId,group)

if(errorCode !=0): 
myIndenter.displayErrorAndClose (socketId, errorCode, 'GroupJogModeEnable')

sys.exit()

count $=$ count +1

myxps.GroupMoveAbort(socketId,group)

[errorCode, returnString]=myxps.PositionerSGammaParametersSet(socketId,positioner, 10,160,.005,.05)

\#myxps.GroupMoveRelative(socketId,group,[-.6])

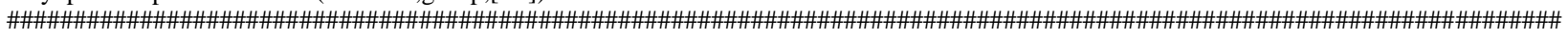

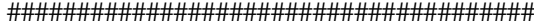

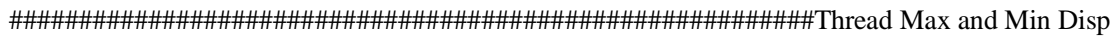

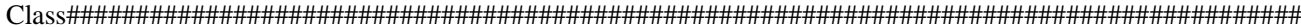

class DispThread (threading.Thread):

\#pause = threading.Event ()

stop $=$ False

def__init__self):

Thread._init_(self)

self.start()

self.stoprequest $=$ threading.Event $($ )

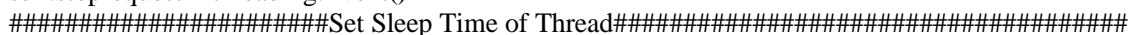

def sleep(self,sec):

time.sleep(sec)

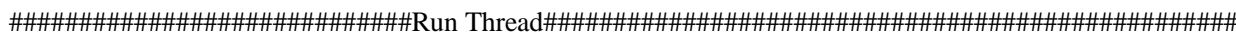

def run(self):

global setMin

global setMax

\#global displayMinForce_box_2

\#global displayMinDisp_box_2

\#global dispMaxDisp_box_2

\#global dispMaxForce_box_2

\#global pointOfContact_1

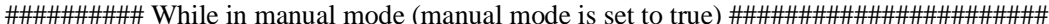

while(dispMode):

dispVal_array $=[]$

\#Limit number of displayed values

if (not(setMin) and not(setMax) ):

currentForce $=$ loadCellCalibrateVoltage(myxps.GPIOAnalogGet(socketId,["GPIO2.ADC1"])[1])*1000

displacement_Value $=$ myxps.GroupPositionCurrentGet(socketId,positioner,1)[1]-pointOfContact_1

elif(setMin and not(setMax) ):

currentForce $=$ loadCellCalibrateVoltage(myxps.GPIOAnalogGet(socketId,["GPIO2.ADC1"])[1])*1000

displacement_Value $=$ myxps.GroupPositionCurrentGet(socketId,positioner,1)[1]-pointOfContact_1

dispVal_array.append(currentForce)

dispVal_array.append(displacement_Value)

wx.CallAfter(pub.sendMessage,"Disp_Val",disp_update = dispVal_array)

time.sleep(.3)

\#wid.Refresh()

class PicturePopUp(wx.Frame):

def__init_(self):

wx.Frame._init_ (self,None,id = wx.ID_ANY,title = "Experiment Plots", pos = wx.DefaultPosition, size $=(500,400)$, style =

wx.DEFAULT_FRAME_STYLE|wx.TAB_TRAVERSAL )

popUpSizer $=$ wx.FlexGridSizer $(0,1,0,0)$

popUpSizer.SetFlexibleDirection(wx.BOTH )

popUpSizer.SetNonFlexibleGrowMode( wx.FLEX_GROWMODE_SPECIFIED )

popUpSizer.SetMinSize( wx.Size $(500,400))$

global plotScroll

global plotSizer

plotScroll = wx.ScrolledWindow $($ self, wx.ID_ANY, wx.DefaultPosition, wx.DefaultSize, wx.HSCROLL|wx.VSCROLL )

plotScroll.SetScrollRate( 5, 5 )

plotScroll.SetMinSize( wx.Size(500,400 ) )

plotSizer $=w x$. FlexGridSizer $(0,1,0,0)$

plotSizer.SetFlexibleDirection( wx.VERTICAL )

plotSizer.SetNonFlexibleGrowMode( wx.FLEX_GROWMODE_SPECIFIED )

plotSizer.SetMinSize( wx.Size(500,400 ) ) 
plotScroll.SetSizer( plotSizer )

plotScroll.Layout()

plotSizer.Fit( plotScroll )

popUpSizer.Add( plotScroll, 1, wx.EXPAND|wx.ALL|wx.ALIGN_CENTER_VERTICAL|wx.ALIGN_CENTER_HORIZONTAL, 5 )

self.SetSizer( popUpSizer )

self.Layout()

self.Centre( wx.BOTH )

def__del_( self ):

pass

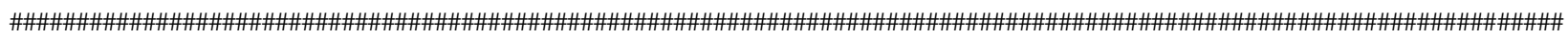
\#\#\#\#\#\#\#\#\#\#\#\#\#\#\#\#\#\#\#\#\#\#\#\#\#\#\#\#\#\#\#\#\#\#

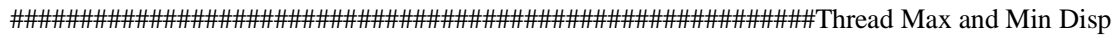

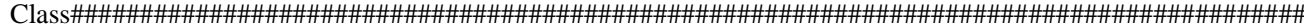

class loadCell (threading. Thread):

\#pause $=$ threading. Event ()

stop $=$ False

def__init__(self):

Thread._init__(self)

self.start()

self.stoprequest $=$ threading.Event $($ )

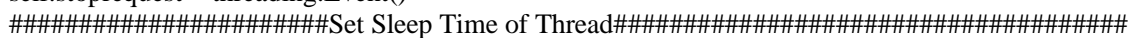

def sleep (self,sec):

time.sleep $(\mathrm{sec})$

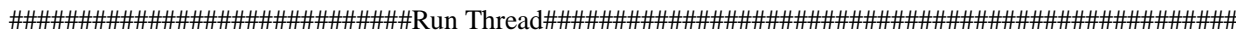

def run(self):

\#global cw

\#global wid

global Alone

Alone $=$ True

while Alone:

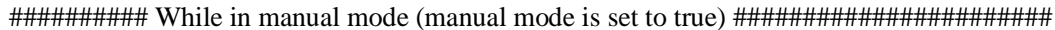

cw_val = loadCellCalibrateVoltage(myxps.GPIOAnalogGet(socketId,["GPIO2.ADC1"])[1])*1000

wx.CallAfter(pub.sendMessage,"cw",cw_update = cw_val)

time.sleep $(.3)$

\#wid.Refresh()

class Widget(wx.Panel):

def__init__(self, parent, id):

wx.Panel.__init_(self, parent, id, size $=(-1,30)$, style=wx.SUNKEN_BORDER)

self.parent $=$ parent

self.font $=$ wx.Font $(9$, wx.FONTFAMILY_DEFAULT, wx.FONTSTYLE_NORMAL,

wx.FONTWEIGHT_NORMAL, False, 'Courier 10 Pitch')

self.Bind(wx.EVT_PAINT, self.OnPaint)

self.Bind(wx.EVT_SIZE, self.OnSize)

def OnPaint(self, event):

global peakRange

global cw

num $=$ np.logspace $(0,1$, num $=7)$

numHigh $=[]$

numMid $=[]$

low $=1$

$\#$ high = math.log $(1500,10)$

\#difference $=($ float $($ high $)-$ low $) /$ float $(7)$

difference $=$ float $(75) / 7$

\#print difference

for $\mathrm{i}$ in range $(0,8)$ :

numHigh.append(math.log(low, 10)

low $=$ low+difference

mid $=$ float $(345) / 1500 *$ peakRange

differenceHighLow $=$ float $($ peakRange-peakRange $* .05-10) / 4$

for $\mathrm{i}$ in range $(0,4)$ :

numMid.append(mid)

mid $=$ mid+differenceHighLow

ratio $=$ float $($ peakRange $* .05) /$ numHigh[len(numHigh) -1$]$

$\mathrm{dc}=\mathrm{wx}$. PaintDC $($ self $)$ 


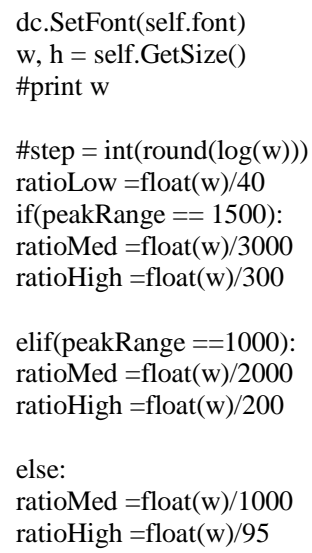

for $\mathrm{x}$ in range( $(0$, len(numHigh)):

numHigh $[\mathrm{x}]=$ numHigh $[\mathrm{x}] *$ ratio+peakRange*.95

$\mathrm{j}=0$

$\#$ till $=(\mathrm{w} / 1500) * \mathrm{cw}$

$\#$ full $=(\mathrm{w} / 1500) * 1500 * .9$

if $\mathrm{cw}<10$ :

till $=$ float $(\mathrm{cw}) *$ ratioLow

dc.SetPen(wx.Pen('\#FFFFB8'))

dc.SetBrush(wx.Brush('\#FFFFB8'))

dc.DrawRectangle $(0,0$, till, 30$)$

elif ( $\mathrm{cw}>10$ and $\mathrm{cw}<(.95 *$ peakRange $)$ ):

till $=$ float $(\mathrm{cw}) *$ ratioMed + num $[$ len(num) -1$] *$ ratioLow

dc.SetPen(wx.Pen('\#FFFFB8'))

dc.SetBrush(wx.Brush('\#FFFFB8'))

dc.DrawRectangle $(0,0$, till, 30)

else:

full = numMid[len(numMid)-1]*ratioMed+num[len(num)-1]*ratioLow

till $=\mathrm{cw}^{*}$ ratioHigh-(numHigh $[0] *$ ratioHigh-numMid[len(numMid)-1 ${ }^{*}$ ratioMed-num[len(num)-1]*ratioLow)

dc.SetPen(wx.Pen('\#FFFFB8'))

dc.SetBrush(wx.Brush('\#FFFFB8'))

dc.DrawRectangle $(0,0$, full, 30$)$

dc.SetPen(wx.Pen('\#ffafaf'))

dc.SetBrush(wx.Brush('\#ffafaf'))

dc.DrawRectangle(full, 0, till-full, 30)

dc.SetPen(wx.Pen('\#5C5142'))

for $i$ in range $(0$, len(num)):

dc.DrawLine(num[i]*ratioLow, 0, num[i]*ratioLow, 6)

width, height $=$ dc.GetTextExtent $(\operatorname{str}(\{0: .0 f\}$ '.format $($ num $[i])))$

dc.DrawText(str('\{0:.0f\}'.format(num[i])), num[i]*ratioLow-width/2, 6)

for $\mathrm{i}$ in range(0,len(numMid)):

dc.DrawLine(num[len(num)-1]*ratioLow+numMid[i]*ratioMed, 0, num[len(num)-1]*ratioLow+numMid[i]*ratioMed, 6)

if(i<3):

width, height $=$ dc.GetTextExtent $(\operatorname{str}('\{0: .0 f\}$ '.format $($ numMid $[i])))$

dc.DrawText(str('\{0:.0f\}'.format(numMid[i])), numMid[i]*ratioMed+num[len(num)-1]*ratioLow-width/2, 6)

\#print numHigh[]*ratioHigh-numMid[len(numMid)-1]*ratioMed-num[len(num)-1]*ratioLow

\#print numHigh[2]*ratioHigh-(numHigh[2]*ratioHigh-numMid[len(numMid)-1]*ratioMed-num[len(num)-1]*ratioLow)

for $i$ in range( 0 ,len(numHigh)):

\#dc.DrawLine(num[len(num)-1]*ratioLow+numMid[len(numMid)-1]*ratioMed+numHigh[i]*ratioHigh, 0, num[len(num)-

1]*ratioLow+numMid[len(numMid)-1]*ratioMed+numHigh[i]*ratioHigh, 6)

dc.DrawLine(round(numHigh[i]*ratioHigh-(numHigh[0]*ratioHigh-numMid[len(numMid)-1]*ratioMed-num[len(num)-1]*ratioLow)), 0, round(numHigh[i]*ratioHigh-(numHigh[0]*ratioHigh-numMid[len(numMid)-1]*ratioMed-num[len(num)-1]*ratioLow)), 6)

if $(\mathrm{i}<2$ or $\mathrm{i}==4)$ :

width, height $=$ dc.GetTextExtent $(\operatorname{str}(\{0: .0 f\}$ '.format $($ numHigh $[i])))$

dc.DrawText(str('\{0:.0f $\}$ '.format(numHigh[i])), round(numHigh[i]*ratioHigh-(numHigh[0]*ratioHigh-numMid[len(numMid)-1]*ratioMednum[len(num)-1]*ratioLow))-width/2,6) 
def OnSize(self, event):

self.Refresh()

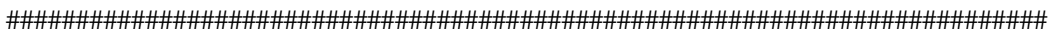

\#\# Class UVAColumbiaInterface

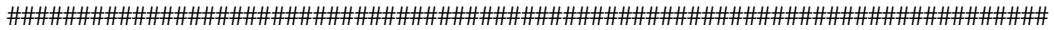

class UVAColumbiaInterface ( wx.Frame ):

def__init_( self ):

global displayMinForce_box_2

global displayMinDisp_box_2

global dispMaxDisp_box_2

global dispMaxForce_box_2

global save_log_dialogFileName

global wid

global potent_Mode

wx.Frame._init_ $($ self, None, id = wx.ID_ANY, title $=$ u"University of Virginia Force and Displacement Controlled Indenter", pos $=$

wx.DefaultPosition, $\operatorname{size}=w x \cdot \operatorname{Size}(1100,780)$, style $=$ wx.DEFAULT_FRAME_STYLE|wx.TAB_TRAVERSAL $)$

self.SetSizeHintsSz( wx.DefaultSize, wx.DefaultSize )

UISizer $=$ wx.FlexGridSizer $(1,2,0,0)$

UISizer.SetFlexibleDirection( wx.BOTH )

UISizer.SetNonFlexibleGrowMode( wx.FLEX_GROWMODE_SPECIFIED)

self.UI_Panel $=$ wx.Panel $($ self, wx.ID_ANY, wx.DefaultPosition, wx.DefaultSize, wx.TAB_TRAVERSAL )

self.UI_Panel.SetBackgroundColour(wx.SystemSettings.GetColour( wx.SYS_COLOUR_BTNTEXT ) )

topandBottomSizer $=$ wx.FlexGridSizer $(2,1,0,0)$

topandBottomSizer.SetFlexibleDirection( wx.VERTICAL )

topandBottomSizer.SetNonFlexibleGrowMode( wx.FLEX_GROWMODE_SPECIFIED )

self.headerPanel = wx.Panel( self.UI_Panel, wx.ID_ANY, wx.DefaultPosition, wx.Size (1100,105 ), wx.TAB_TRAVERSAL )

StepTitleSizer $=$ wx.GridBagSizer $(0,0)$

StepTitleSizer.SetFlexibleDirection( wx.BOTH )

StepTitleSizer.SetNonFlexibleGrowMode( wx.FLEX_GROWMODE_SPECIFIED )

\# StepTitleSizer.SetMinSize( wx.Size (1100,135))

self.leftButtonPanel $=$ wx.Panel( self.headerPanel, wx.ID_ANY, wx.DefaultPosition, wx.DefaultSize, wx.TAB_TRAVERSAL )

leftButton_sizer $=$ wx.GridBagSizer $(0,0)$

leftButton_sizer.SetFlexibleDirection(wx.BOTH)

leftButton_sizer.SetNonFlexibleGrowMode( wx.FLEX_GROWMODE_SPECIFIED )

self.leftButton_panel = wx.Panel $($ self.leftButtonPanel, wx.ID_ANY, wx.DefaultPosition, wx.DefaultSize, wx.TAB_TRAVERSAL )

leftButton_interiorSizer $=$ wx.BoxSizer $(w x$.VERTICAL $)$

scaled_NoButton $=$ self.scale_bitmap (wx.Bitmap ( "GUI_Images/NoButton.bmp", wx.BITMAP_TYPE_ANY ),200,100)

self.NoButton_Left $=$ wx.BitmapButton ( self.leftButton_panel, wx.ID_ANY, scaled_NoButton, wx.DefaultPosition, wx.Size $(-1,-1)$,

wx.BU_AUTODRAW )

leftButton_interiorSizer.Add( self.NoButton_Left, 0, wx.EXPAND, 5 )

scaled_Step1_Left = self.scale_bitmap(wx.Bitmap( "GUI_Images/Step1_Left.bmp", wx.BITMAP_TYPE_ANY ),200,100)

self.Step1_Left $=$ wx.BitmapButton( self.leftButton_panel, 1, scaled_Step1_Left, wx.DefaultPosition, wx.DefaultSize, wx.BU_AUTODRAW )

self.Step1_Left.Enable( False )

self.Step1_Left.Hide()

self.Bind(wx.EVT_BUTTON,self.changePagesLeft,id=1)

leftButton_interiorSizer.Add( self.Step1_Left, 0, 0, 5 )

scaled_Step2_Left $=$ self.scale_bitmap(wx.Bitmap( "GUI_Images/Step2_Left.bmp", wx.BITMAP_TYPE_ANY ),200,100)

self.Step2_Left $=$ wx.BitmapButton( self.leftButton_panel, 2,scaled_Step2_Left, wx.DefaultPosition, wx.DefaultSize, wx.BU_AUTODRAW ) self.Step2_Left.Enable( False )

self.Step2_Left.Hide()

self.Bind(wx.EVT_BUTTON,self.changePagesLeft,id=2)

leftButton_interiorSizer.Add( self.Step2_Left, 0, 0, 5 )

scaled_Step3_Left $=$ self.scale_bitmap(wx.Bitmap( "GUI_Images/Step3_Left.bmp", wx.BITMAP_TYPE_ANY ),200,100)

self.Step3_Left = wx.BitmapButton( self.leftButton_panel, 3,scaled_Step3_Left, wx.DefaultPosition, wx.DefaultSize, wx.BU_AUTODRAW ) self.Step3_Left.Enable( False )

self.Step3_Left.Hide()

self.Bind(wx.EVT_BUTTON,self.changePagesLeft,id=3)

leftButton_interiorSizer.Add( self.Step3_Left, $0,0,5$ )

scaled_Step4_Left $=$ self.scale_bitmap(wx.Bitmap( "GUI_Images/Step4_Left.bmp", wx.BITMAP_TYPE_ANY ),200,100)

self.Step4_Left $=$ wx.BitmapButton( self.leftButton_panel, 22, scaled_Step4_Left, wx.DefaultPosition, wx.DefaultSize, wx.BU_AUTODRAW ) self.Step4_Left.Enable( False ) 
self.Step4_Left.Hide()

self.Bind(wx.EVT_BUTTON,self.changePagesLeft,id=22)

leftButton_interiorSizer.Add( self.Step4_Left, 0, 0, 5 )

scaled_Step5_Left $=$ self.scale_bitmap(wx.Bitmap( "GUI_Images/Step5_Left.bmp", wx.BITMAP_TYPE_ANY ),200,100)

self.Step5_Left $=$ wx.BitmapButton( self.leftButton_panel, 4, scaled_Step5_Left, wx.DefaultPosition, wx.DefaultSize, wx.BU_AUTODRAW ) self.Step5_Left.Enable( False )

self.Step5_Left.Hide()

self.Bind(wx.EVT_BUTTON,self.changePagesLeft,id=4)

leftButton_interiorSizer.Add( self.Step5_Left, 0, 0, 5 )

self.leftButton_panel.SetSizer( leftButton_interiorSizer )

self.leftButton_panel.Layout()

leftButton_interiorSizer.Fit( self.leftButton panel )

leftButton_sizer.Add( self.leftButton_panel, wx.GBPosition( 0, 0 ), wx.GBSpan( 1, 1), wx.EXPAND, 5 )

self.leftButtonPanel.SetSizer( leftButton_sizer)

self.leftButtonPanel.Layout()

leftButton_sizer.Fit( self.leftButtonPanel )

StepTitleSizer.Add( self.leftButtonPanel, wx.GBPosition( 0, 0 ), wx.GBSpan( 1, 1 ), wx.EXPAND, 5 )

self.step_panel $=$ wx.Panel $($ self.headerPanel, wx.ID_ANY, wx.DefaultPosition, wx.Size (650,100 ), wx.TAB_TRAVERSAL )

step_sizer $=$ wx.BoxSizer $($ wx.VERTICAL $)$

self.upperStep_text $=$ wx.StaticText( self.step_panel, wx.ID_ANY, "Step 1: Approach Surface and Find Point of Contact", wx.DefaultPosition, wx.DefaultSize, wx.ALIGN_CENTRE )

self.upperStep_text.Wrap( -1 )

self.upperStep_text.SetFont( wx.Font( 26, 74, 90, 92, False, "Calibri" ) )

self.upperStep_text.SetForegroundColour( wx.SystemSettings.GetColour( wx.SYS_COLOUR_INFOBK ) )

step_sizer.Add( self.upperStep_text, 1, wx.ALIGN_CENTER_HORIZONTAL|wx.RIGHT|wx.LEFT, 5 )

self.step_panel.SetSizer( step_sizer )

self.step_panel.Layout()

StepTitleSizer.Add( self.step_panel, wx.GBPosition( 0, 1 ),wx.GBSpan(1,3), wx.EXPAND|wx.ALL, 5 )

self.rightButtonPanel = wx.Panel( self.headerPanel, wx.ID_ANY, wx.DefaultPosition, wx.DefaultSize, wx.TAB_TRAVERSAL )

rightButtonSizer $=$ wx. GridBagSizer $(0,0)$

rightButtonSizer.SetFlexibleDirection( wx.BOTH )

rightButtonSizer.SetNonFlexibleGrowMode( wx.FLEX_GROWMODE_SPECIFIED )

self.rightButtonInteriorPanel $=$ wx.Panel $($ self.rightButtonPanel, wx.ID_ANY, wx.DefaultPosition, wx.DefaultSize, wx.TAB_TRAVERSAL )

RightButtonInteriorSizer $=$ wx.BoxSizer $(w x$. HORIZONTAL $)$

scaled_Step2_Right $=$ self.scale_bitmap(wx.Bitmap( "GUI_Images/Step2_Right.bmp", wx.BITMAP_TYPE_ANY ),200,100)

self.Step2_Right $=$ wx.BitmapButton( self.rightButtonInteriorPanel, 5, scaled_Step2_Right, wx.DefaultPosition, wx.DefaultSize, wx.BU_AUTODRAW )

RightButtonInteriorSizer.Add( self.Step2_Right, 0, 0, 5 )

self.Bind(wx.EVT_BUTTON,self.changePagesRight,id=5)

scaled_Step3_Right $=$ self.scale_bitmap(wx.Bitmap( "GUI_Images/Step3_Right.bmp", wx.BITMAP_TYPE_ANY ),200,100)

self.Step3_Right $=$ wx.BitmapButton( self.rightButtonInteriorPanel, 6,scaled_Step3_Right, wx.DefaultPosition, wx.DefaultSize,

wx.BU_AUTODRAW )

self.Step3_Right.Enable( False )

self.Step3_Right.Hide()

self.Bind(wx.EVT_BUTTON,self.changePagesRight,id=6)

RightButtonInteriorSizer.Add( self.Step3_Right, 0, 0, 5 )

scaled_Step4_Right $=$ self.scale_bitmap(wx.Bitmap( "GUI_Images/Step4_Right.bmp", wx.BITMAP_TYPE_ANY ),200,100)

self.Step4_Right $=$ wx.BitmapButton( self.rightButtonInteriorPanel, 7, scaled_Step4_Right, wx.DefaultPosition, wx.DefaultSize,

wx.BU_AUTODRAW )

self.Step4_Right.Enable( False )

self.Step4_Right.Hide()

self.Bind(wx.EVT_BUTTON,self.changePagesRight,id=7)

RightButtonInteriorSizer.Add( self.Step4_Right, 0, 0, 5)

scaled_NoButton_Right $=$ self.scale_bitmap(wx.Bitmap ( "GUI_Images/NoButton.bmp", wx.BITMAP_TYPE_ANY ),200,100)

self.NoButton = wx.BitmapButton $($ self.rightButtonInteriorPanel, 8,scaled_NoButton_Right, wx.DefaultPosition, wx.DefaultSize, wx.BU_AUTODRAW )

self.NoButton.Enable( False )

self.NoButton.Hide()

self.Bind(wx.EVT_BUTTON,self.changePagesRight,id=8)

RightButtonInteriorSizer.Add( self.NoButton, 0, 0, 5 )

scaled_Step6_Right = self.scale_bitmap(wx.Bitmap( "GUI_Images/Step6_Right.bmp", wx.BITMAP_TYPE_ANY ),200,100) 
self.Step6_Right $=$ wx.BitmapButton $($ self.rightButtonInteriorPanel, 9, scaled_Step6_Right, wx.DefaultPosition, wx.DefaultSize, wx.BU_AUTODRAW )

self.Step6_Right.Enable( False )

self.Step6_Right.Hide()

self.Bind(wx.EVT_BUTTON,self.changePagesRight,id=9)

RightButtonInteriorSizer.Add( self.Step6_Right, 0, 0, 5 )

self.rightButtonInteriorPanel.SetSizer( RightButtonInteriorSizer )

self.rightButtonInteriorPanel.Layout()

RightButtonInteriorSizer.Fit( self.rightButtonInteriorPanel )

rightButtonSizer.Add( self.rightButtonInteriorPanel, wx.GBPosition( 0, 0 ), wx.GBSpan( 1, 1 ), wx.EXPAND, 5 )

self.rightButtonPanel.SetSizer( rightButtonSizer )

self.rightButtonPanel.Layout()

rightButtonSizer.Fit( self.rightButtonPanel )

StepTitleSizer.Add( self.rightButtonPanel, wx.GBPosition( 0, 4 ), wx.GBSpan( 1, 1 ), 0, 5 )

self.headerPanel.SetSizer( StepTitleSizer )

self.headerPanel.Layout()

topandBottomSizer.Add( self.headerPanel, 1, wx.EXPAND |wx.ALL, 5 )

self.decisionAndLogPanel = wx.Panel( self.UI_Panel, wx.ID_ANY, wx.DefaultPosition, wx.Size( 1100,630$)$, wx.TAB_TRAVERSAL $)$

decision_LogSizer $=$ wx.FlexGridSizer $(1,2,0,0)$

decision_LogSizer.SetFlexibleDirection(wx.HORIZONTAL )

decision_LogSizer.SetNonFlexibleGrowMode( wx.FLEX_GROWMODE_SPECIFIED )

self.decisionPanel $=$ wx.Panel $($ self.decisionAndLogPanel, wx.ID_ANY, wx.DefaultPosition, wx.DefaultSize, wx.TAB_TRAVERSAL )

self.decisionPanel.SetBackgroundColour( wx.SystemSettings.GetColour( wx.SYS_COLOUR_3DLIGHT ) )

self.decisionPanel.SetMinSize( wx.Size (660,-1))

self.decisionPanel.SetMaxSize( wx.Size $(660,-1)$ )

decisionSizer $=$ wx.BoxSizer $(w x$. VERTICAL $)$

decisionSizer.SetMinSize( wx.Size( 660,-1 ))

self.step1_panel = wx.Panel( self.decisionPanel, wx.ID_ANY, wx.DefaultPosition, wx.DefaultSize, wx.TAB_TRAVERSAL )

step1_sizer =wx.FlexGridSizer $(3,1,0,0)$

step1_sizer.SetFlexibleDirection(wx.VERTICAL )

step1_sizer.SetNonFlexibleGrowMode( wx.FLEX_GROWMODE_SPECIFIED )

step1_sizer.SetMinSize( wx.Size (660,600))

self.forceRange_text_1 =wx.Panel( self.step1_panel, wx.ID_ANY, wx.DefaultPosition, wx.DefaultSize, wx.SIMPLE_BORDER )

forceRange_sizer_1 $=$ wx.FlexGridSizer $(3,1,0,0)$

forceRange_sizer_1.SetFlexibleDirection(wx.BOTH )

forceRange_sizer_1.SetNonFlexibleGrowMode( wx.FLEX_GROWMODE_SPECIFIED )

forceRangeTextSizer_1 $=$ wx.FlexGridSizer $(0,3,0,0)$

forceRangeTextSizer_1.SetFlexibleDirection(wx.BOTH )

forceRangeTextSizer_1.SetNonFlexibleGrowMode( wx.FLEX_GROWMODE_SPECIFIED )

forceRangeTextSizer_1.SetMinSize( wx.Size $(660,-1)$ )

self.approachSurface_text_1 = wx.StaticText( self.forceRange_text_1, wx.ID_ANY, u"Approach surface:", wx.DefaultPosition, wx.Size( $-1,30$ ), 0 )

self.approachSurface_text_1.Wrap( -1 )

self.approachSurface_text_1.SetFont( wx.Font( 14, 74, 90, 92, False, "Calibri" ) )

forceRangeTextSizer_1.Add( self.approachSurface_text_1, 0, wx.LEFT|wx.RIGHT, 5 )

self.engageJoystick_text_1 = wx.StaticText( self.forceRange_text_1, wx.ID_ANY, u"Engage joystick", wx.DefaultPosition, wx.Size( 475,30 ), 0

self.engageJoystick_text_1.Wrap( -1 )

self.engageJoystick_text_1.SetFont( wx.Font( 14, 74, 90, 90, False, "Calibri" ) )

forceRangeTextSizer_1.Add( self.engageJoystick_text_1, 0, wx.LEFT|wx.RIGHT, 5 )

forceRange_sizer_1.Add( forceRangeTextSizer_1, 1, wx.EXPAND, 5 )

velocity_sizer_1 $=$ wx.GridBagSizer $(0,0)$

velocity_sizer_1.SetFlexibleDirection( wx.VERTICAL )

velocity_sizer_1.SetNonFlexibleGrowMode( wx.FLEX_GROWMODE_SPECIFIED )

velocity_sizer_1.SetMinSize(wx.Size( $300,-1)$ )

self.min_text_app_1 $=$ wx.StaticText( self.forceRange_text_1, wx.ID_ANY, u"Min", wx.DefaultPosition, wx.DefaultSize, 0 )

self.min_text_app_1.Wrap $(-1)$

self.min_text_app_1.SetFont( wx.Font( 12, 74, 90, 90, False, "Calibri" ) )

self.min_text_app_1.SetForegroundColour((96,96,96))

velocity_sizer_1.Add( self.min_text_app_1, wx.GBPosition( 0,0 ), wx.GBSpan $(1,1)$,

wx.LEFT|wx.RIGHT|wx.ALIGN_CENTER_VERTICAL|wx.ALIGN_CENTER_HORIZONTAL, 5 )

self.vel_text_app_1 $=$ wx.StaticText( self.forceRange_text_1, wx.ID_ANY, u"Velocity $(\mathrm{mm} / \mathrm{s})$ ", wx.DefaultPosition, wx.DefaultSize,

wx.ALIGN_CENTRE )

self.vel_text_app_1.Wrap ( -1$)$

self.vel_text_app_1.SetFont( wx.Font (12, 74, 90, 90, False, "Calibri" ) )

self.vel_text_app_1.SetMinSize( wx.Size $(150,-1)$ )

velocity_sizer_1.Addd( self.vel_text_app_1, wx.GBPosition( 0,1 ), wx.GBSpan $(1,1)$,

wx.RIGHT|wx.LEFT|wx.ALIGN_BOTTOM|wx.ALIGN_CENTER_HORIZONTAL, 5 ) 
self.max_text_app_1 $=$ wx.StaticText( self.forceRange_text_1, wx.ID_ANY, u"Max", wx.DefaultPosition, wx.DefaultSize,

wx.ALIGN_CENTRE )

self.max_text_app_1.Wrap( -1 )

self.max_text_app_1.SetFont( wx.Font( 12, 74, 90, 90, False, "Calibri" ) )

self.max_text_app_1.SetForegroundColour $((96,96,96))$

velocity_sizer_1.Add( self.max_text_app_1, wx.GBPosition( 0,2 ), wx.GBSpan $(1,1)$,

wx.LEFT|wx.RIGHT|wx.ALIGN_CENTER_HORIZONTAL, 5 )

self.minVal_text_app_1 $=$ wx.StaticText( self.forceRange_text_1, wx.ID_ANY, u"0.1", wx.DefaultPosition, wx.Size $(-1,-1), 0$ )

self.minVal_text_app_1.Wrap( -1 )

self.minVal_text_app_1.SetFont( wx.Font( $12,74,90,90$, False, "Calibri" ) )

self.minVal_text_app_1.SetForegroundColour( $(96,96,96))$

velocity_sizer_1.Add( self.minVal_text_app_1, wx.GBPosition( 1,0 ), wx.GBSpan( 1,1 ),

wx.LEFT|wx.RIGHT|wx.ALIGN_CENTER_HORIZONTAL, 5 )

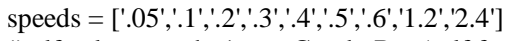

\#self.vel_appoach_1=wx.ComboBox(self.forceRange text 1 ,value $={ }^{\prime} .3^{\prime}$, choices $=$ speeds, style $=$ wx.CB_READONLY,wx.DefaultPosition)

self.vel_appoach_1=wx.ComboBox(self.forceRange_text_1, value $=$ ' 2.4 ', choices $=$ speeds, style $=$ wx.CB_READONLY)\#, wx.DefaultPosition)

self.vel_appoach_1.SetFont( wx.Font( 14, 74, 90, 90, False, "Calibri" ) )

self.vel_appoach_1.SetForegroundColour(wx.SystemSettings.GetColour( wx.SYS_COLOUR_BTNTEXT ))

self.vel_appoach_1.SetBackgroundColour(wx.Colour( 185, 185, 185 ))

self.vel_appoach_1.SetMinSize(wx.Size (100,-1 ))

self.vel_appoach_1.Bind(wx.EVT_TEXT,self.UpdateSpeed)

velocity_sizer_1.Add( self.vel_appoach_1, wx.GBPosition( 1,1 ), wx.GBSpan( 1,1 ),

wx.ALIGN_CENTER_HORIZONTAL|wx.RIGHT|wx.LEFT, 5 )

self.maxVal_text_app_1 $=$ wx.StaticText( self.forceRange_text_1, wx.ID_ANY, u"2", wx.DefaultPosition, wx.DefaultSize, 0 )

self.maxVal_text_app_1.Wrap( -1 )

self.maxVal_text_app_1.SetFont( wx.Font( 12, 74, 90, 90, False, "Calibri" ) )

self.maxVal_text_app_1.SetForegroundColour( $(96,96,96))$

velocity_sizer_1.Add( self.maxVal_text_app_1,wx.GBPosition( 1,2 ), wx.GBSpan( 1,1),

wx.ALL/wx.ALIGN_CENTER_VERTICAL|wx.ALIGN_CENTER_HORIZONTAL, 5 )

forceRange_sizer_1.Add( velocity_sizer_1, 1, wx.ALIGN_CENTER_HORIZONTAL, 5 )

button approach $\_$sizer $=$wx. GridSizer $(0,2,0,0)$

self.Engage_Button = wx.Button( self.forceRange_text_1, 10, u"Engage", wx.DefaultPosition, wx.Size ( 175,60 ), 0 )

self.Engage_Button.SetFont( wx.Font (18, 74, 90, 92, False, "Calibri" ) )

self.Bind(wx.EVT_BUTTON, self.Engage,id=10)

button_approach_sizer.Add( self.Engage_Button, 0, wx.ALL|wx.ALIGN_RIGHT, 5 )

self.Disengage_Button = wx.Button( self.forceRange_text_1, 11, u"Disengage", wx.DefaultPosition, wx.Size $(175,60$ ), 0 )

self.Disengage_Button.SetFont( wx.Font( 18, 74, 90, 92, False, "Calibri" ) )

self.Disengage_Button.SetForegroundColour(wx.SystemSettings.GetColour( wx.SYS_COLOUR_BTNHIGHLIGHT ) )

self.Disengage_Button.SetBackgroundColour( wx.SystemSettings.GetColour( wx.SYS_COLOUR_3DDKSHADOW ) )

self.Bind(wx.EVT_BUTTON,self.Disengage,id=11)

button_approach_sizer.Add( self.Disengage_Button, 0, wx.TOP|wx.BOTTOM|wx.LEFT, 5 )

forceRange_sizer_1.Add( button_approach_sizer, 0, wx.EXPAND|wx.ALIGN_CENTER_HORIZONTAL|wx.ALIGN_CENTER_VERTICAL, 5 )

self.forceRange_text_1.SetSizer(forceRange_sizer_1 )

self.forceRange_text_1.Layout()

forceRange_sizer_1.Fit( self.forceRange_text_1 )

step1_sizer.Add( self.forceRange_text_1, 1, wx.EXPAND, 5 )

self.findPOC_panel_1 $=$ wx.Panel( self.step1_panel, wx.ID_ANY, wx.DefaultPosition, wx.Size( 660,375), wx.SIMPLE_BORDER )

findPOC_sizer_1 $=$ wx.FlexGridSizer $(3,1,0,0)$

findPOC_sizer_1.SetFlexibleDirection(wx.BOTH)

findPOC_sizer_1.SetNonFlexibleGrowMode( wx.FLEX_GROWMODE_SPECIFIED )

textPOC_sizer_1 $=$ wx.FlexGridSizer $(0,2,0,0)$

textPOC_sizer_1.SetFlexibleDirection(wx.HORIZONTAL)

textPOC_sizer_1.SetNonFlexibleGrowMode( wx.FLEX_GROWMODE_SPECIFIED )

textPOC_sizer_1.SetMinSize( wx.Size $(660,-1)$ )

self.findPOC_text_1 = wx.StaticText( self.findPOC_panel_1, wx.ID_ANY, u"Find first point of contact:", wx.DefaultPosition, wx.DefaultSize, 0 )

self.findPOC_text_1.Wrap( -1$)$

self.findPOC_text_1.SetFont( wx.Font( 14, 74, 90, 92, False, "Calibri" ) )

textPOC_sizer_1.Add( self.findPOC_text_1, 0, wx.ALL, 5 )

self.indentParm_text_1 $=$ wx.StaticText( self.findPOC_panel_1, wx.ID_ANY, u"Indentation parameters", wx.DefaultPosition, wx.DefaultSize, 0 )

self.indentParm_text_1.Wrap(-1)

self.indentParm_text_1.SetFont( wx.Font( 14, 74, 90, 90, False, "Calibri" ) )

self.indentParm_text_1.SetMinSize(wx.Size( 450,-1 ))

textPOC_sizer_1.Add( self.indentParm_text_1, 0, wx.TOP|wx.RIGHT|wx.LEFT, 5 ) 
findPOC_sizer_1.Add( textPOC_sizer_1, 1, wx.EXPAND, 5 )

POC_sizer_21 = wx.GridBagSizer $(0,0)$

POC_sizer_21.SetFlexibleDirection(wx.BOTH )

POC_sizer_21.SetNonFlexibleGrowMode( wx.FLEX_GROWMODE_SPECIFIED )

self.max_text_21 = wx.StaticText( self.findPOC_panel_1, wx.ID_ANY, u"Max", wx.DefaultPosition, wx.DefaultSize, 0 )

self.max_text_21.Wrap( -1 )

self.max_text_21.SetFont( wx.Font( 12, 74, 90, 90, False, "Calibri" ) )

self.max_text_21.SetForegroundColour( $(96,96,96))$

POC_sizer_21.Add( self.max_text_21, wx.GBPosition( 0,2), wx.GBSpan( 1,1 ),

wx.ALIGN_RIGHT|wx.ALIGN_CENTER_VERTICAL|wx.BOTTOM|wx.RIGHT|wx.LEFT, 5 )

self.displacement_text_21 = wx.StaticText( self.findPOC_panel_1, wx.ID_ANY, u"displ. (mm)", wx.DefaultPosition, wx.DefaultSize,

wx.ALIGN_LEFT )

self.displacement_text_21.Wrap( -1 )

self.displacement_text_21.SetFont( wx.Font( $12,74,90,90$, False, "Calibri" ) )

POC_sizer_21.Add( self.displacement_text_21, wx.GBPosition( 0,1 ), wx.GBSpan( 1,1 ),

wx.LEFT|wx.RIGHT|wx.TOP|wx.ALIGN_BOTTOM|wx.ALIGN_LEFT, 5 )

self.min_text_21 = wx.StaticText( self.findPOC_panel_1, wx.ID_ANY, u"Min", wx.DefaultPosition, wx.DefaultSize, wx.ALIGN_CENTRE )

self.min_text_21.Wrap $(-1)$

self.min_text_21.SetFont( wx.Font( 12, 74, 90, 90, False, "Calibri" ) )

self.min_text_21.SetForegroundColour( $(96,96,96))$

POC_sizer_21.Add( self.min_text_21, wx.GBPosition( 0,0$)$, wx.GBSpan $(1,1)$,

wx.ALIGN_LEFT|wx.ALIGN_CENTER_VERTICAL|wx.BOTTOM|wx.RIGHT, 5 )

self.minDisp_text_21 = wx.StaticText( self.findPOC_panel_1, wx.ID_ANY, u"0.01", wx.DefaultPosition, wx.Size( $-1,-1$ ), wx.ALIGN_LEFT )

self.minDisp_text_21.Wrap( -1$)$

self.minDisp_text_21.SetFont( wx.Font( 12, 74, 90, 90, False, "Calibri" ) )

self.minDisp_text_21.SetForegroundColour( $(96,96,96))$

self.minDisp_text_21.SetMinSize(wx.Size (100,-1 ))

POC_sizer_21.Add( self.minDisp_text_21, wx.GBPosition( 1,0 ), wx.GBSpan( 1,1 ),

wx.ALIGN_LEFT|wx.TOP|wx.BOTTOM|wx.ALIGN_CENTER_VERTICAL, 5 )

self.disp_spin_21 = FS.FloatSpin( self.findPOC_panel_1, -1 , value $=.6, \mathrm{~min} \_$val $=.01$, max_val=1,increment $=.01$,agwStyle=FS.FS_RIGHT )

self.disp_spin_21.SetFormat("\%f")

self.disp_spin_21.SetDigits(2)

self.disp_spin_21.SetFont( wx.Font( 12, 74, 90, 90, False, "Calibri" ) )

self.disp_spin_21.SetForegroundColour(wx.SystemSettings.GetColour( wx.SYS_COLOUR_BTNTEXT ) )

self.disp_spin_21.SetBackgroundColour( wx.SystemSettings.GetColour( wx.SYS_COLOUR_BTNSHADOW ) )

POC_sizer_21.Add( self.disp_spin_21, wx.GBPosition( 1,1 ), wx.GBSpan $(1,1)$,

wx.ALIGN_CENTER_VERTICAL_|wx.ALIGN_CENTER_HORIZONTAL|wx.RIGHT|wx.LEFT|wx.BOTTOM, 5 )

self.minVel_text_21 = wx.StaticText( self.findPOC_panel_1, wx.ID_ANY, u"1.0", wx.DefaultPosition, wx.Size ( $-1,-1$ ), wx.ALIGN_CENTRE )

self.minVel_text_21.Wrap( -1 )

self.minVel_text_21.SetFont( wx.Font( 12, 74, 90, 90, False, "Calibri" ) )

self.minVel_text_21.SetForegroundColour( $(96,96,96))$

POC_sizer_21.Add( self.minVel_text_21, wx.GBPosition( 3, 0 ), wx.GBSpan ( 1, 1 ),

wx.TOP|wx.BOTTOM|wx.ALIGN_CENTER_VERTICAL|wx.ALIGN_LEFT, 5 )

self.max_dispText_21 = wx.StaticText( self.findPOC_panel_1, wx.ID_ANY, u"5", wx.DefaultPosition,wx.DefaultSize,wx.ALIGN_RIGHT )

self.max_dispText_21.Wrap( -1 )

self.max_dispText_21.SetFont( wx.Font( 12, 74, 90, 90, False, "Calibri" ) )

self.max_dispText_21.SetForegroundColour $((96,96,96))$

self.max_dispText_21.SetMinSize( wx.Size $(100,-1))$

POC_sizer_21.Add( self.max_dispText_21, wx.GBPosition( 1,2), wx.GBSpan( 1, 1),wx.LEFT|wx.RIGHT|wx.ALIGN_RIGHT, 5 ) self.vel_text_21 = wx.StaticText( self.findPOC_panel_1, wx.ID_ANY, u"velocity $(\mathrm{mm} / \mathrm{s}) "$, wx.DefaultPosition, wx.DefaultSize, wx.ALIGN_LEFT )

self.vel_text_21.Wrap( -1 )

self.vel_text_21.SetFont(wx.Font( 12, 74, 90, 90, False, "Calibri" ) )

POC_sizer_21.Add( self.vel_text_21, wx.GBPosition( 2,1),wx.GBSpan( 1, 1 ),

wx.ALIGN_BOTTOM|wx.LEFT|wx.RIGHT|wx.ALIGN_LEFT|wx.TOP, 5 )

self.vel_spin_21 = FS.FloatSpin( $\operatorname{self.findPOC} \_$panel_1, -1 , value $=1$, min_val=1, max_val=2, increment $=.2$, agwStyle=FS.FS_RIGHT)

self.vel_spin_21.SetFormat("\%f")

self.vel_spin_21.SetDigits(2)

self.vel_spin_21.SetFont(wx.Font( 12, 74, 90, 90, False, "Calibri" ))

self.vel_spin_21.SetForegroundColour(wx.SystemSettings.GetColour( wx.SYS_COLOUR_BTNTEXT ))

self.vel_spin_21.SetBackgroundColour(wx.SystemSettings.GetColour(wx.SYS_COLOUR_BTNSHADOW ) )

POC_sizer_21.Add( self.vel_spin_21, wx.GBPosition( 3, 1), wx.GBSpan( 1,1 ),

wx.ALIGN_CENTER_HORIZONTAL|wx.RIGHT|wx.LEFT|wx.BOTTOM, 5 )

self.maxVel_text_21 = wx.StaticText( self.findPOC_panel_1, wx.ID_ANY, u"40", wx.DefaultPosition, wx.DefaultSize, 0 )

self.maxVel_text_21.Wrap( -1 )

self.maxVel_text_21.SetFont( wx.Font( 12, 74, 90, 90, False, "Calibri" ) )

self.maxVel_text_21.SetForegroundColour( $(96,96,96))$ 
POC_sizer_21.Add( self.maxVel_text_21,wx.GBPosition( 3, 2), wx.GBSpan( 1,1 ),

wx.LEFT|wx.RIGHT|wx.ALIGN_RIGHT|wx.ALIGN_CENTER_VERTICAL, 5 )

self.acc_text_21 = wx.StaticText( self.findPOC_panel_1, wx.ID_ANY, u"accel. $(\mathrm{mm} / \mathrm{s} / \mathrm{s}) "$, wx.DefaultPosition, wx.DefaultSize,

wx.ALIGN_LEFT )

self.acc_text_21.Wrap ( -1$)$

self.acc_text_21.SetFont( wx.Font( 12, 74, 90, 90, False, "Calibri" ) )

POC_sizer_21.Add( self.acc_text_21, wx.GBPosition( 4, 1 ), wx.GBSpan( 1,1 ),wx.RIGHT|wx.LEFT|wx.ALIGN_RIGHT|wx.TOP, 5 )

self.min_Acc_text_21 =wx.StaticText( self.findPOC_panel_1, wx.ID_ANY, u"1.0", wx.DefaultPosition, wx.DefaultSize, 0 )

self.min_Acc_text_21.Wrap( -1$)$

self.min_Acc_text_21.SetFont( wx.Font( 12, 74, 90, 90, False, "Calibri" ) )

self.min_Acc_text_21.SetForegroundColour( $(96,96,96))$

POC_sizer_21.Add( self.min_Acc_text_21,wx.GBPosition( 5, 0 ), wx.GBSpan( 1,1 ), wx.ALIGN_CENTER_VERTICAL|wx.ALIGN_LEFT, 5

)

self.acc_spin_21 =FS.FloatSpin( self.findPOC_panel_1, -1 , value $=40$, min_val=1, max_val=160.0,increment $=.01$,agwStyle=FS.FS_RIGHT )

self.acc_spin_21.SetFormat("\%f")

self.acc_spin_21.SetDigits(2)

self.acc_spin_21.SetFont( wx.Font( 12, 74, 90, 90, False, "Calibri" ) )

self.acc_spin_21.SetForegroundColour( wx.SystemSettings.GetColour( wx.SYS_COLOUR_BTNTEXT ) )

self.acc_spin_21.SetBackgroundColour(wx.SystemSettings.GetColour( wx.SYS_COLOUR_BTNSHADOW ) )

POC_sizer_21.Add( self.acc_spin 21, wx.GBPosition $(5,1)$, wx.GBSpan $(1,1)$,

wx.RIGHT|wx.LEFT|wx.ALIGN_CENTER_HORIZONTAL|wx.BOTTOM, 5 )

self.max_Acc_text_21 = wx.StaticText( self.findPOC_panel_1, wx.ID_ANY, u"160", wx.DefaultPosition, wx.DefaultSize, 0 )

self.max_Acc_text_21.Wrap( -1 )

self.max_Acc_text_21.SetFont( wx.Font( 12, 74, 90, 90, False, "Calibri" ) )

self.max_Acc_text_21.SetForegroundColour( $(96,96,96))$

POC_sizer_21.Add( self.max_Acc_text_21, wx.GBPosition( 5, 2 ), wx.GBSpan $(1,1)$,

wx.LEFT|wx.RIGHT|wx.ALIGN_CENTER_VERTICAL/wx.ALIGN_RIGHT, 5 )

self.hold_text_21 = wx.StaticText( self.findPOC_panel_1, wx.ID_ANY, u"hold (s) ", wx.DefaultPosition, wx.DefaultSize, wx.ALIGN_LEFT )

self.hold_text_21.Wrap( -1$)$

self.hold_text_21.SetFont( wx.Font( 12, 74, 90, 90, False, "Calibri" ) )

POC_sizer_21.Add( self.hold_text_21, wx.GBPosition( 6,1 ), wx.GBSpan( 1,1 ),

wx.RIGHT|wx.LEFT|wx.TOP|wx.ALIGN_CENTER_VERTICAL|wx.ALIGN_LEFT, 5 )

self.min_holdText_21 = wx.StaticText( self.findPOC_panel_1, wx.ID_ANY, u"0.1", wx.DefaultPosition, wx.DefaultSize, wx.ALIGN_CENTRE

)

self.min_holdText_21.Wrap( -1$)$

self.min_holdText_21.SetFont( wx.Font( 12, 74, 90, 90, False, "Calibri" ) )

self.min_holdText_21.SetForegroundColour( $(96,96,96))$

POC_sizer_21.Add( self.min_holdText_21, wx.GBPosition( 7, 0 ), wx.GBSpan ( 1, 1 ),wx.ALIGN_LEFT|wx.ALIGN_CENTER_VERTICAL, 5 )

self.hold_spin_21 = FS.FloatSpin( self.findPOC_panel_1,-1,min_val=.1, max_val=600.0,increment = .1, agwStyle=FS.FS_RIGHT )

self.hold_spin_21.SetFormat("\%f")

self.hold_spin_21.SetDigits(2)

self.hold_spin_21.SetFont( wx.Font( 12, 74, 90, 90, False, "Calibri" ) )

self.hold_spin_21.SetForegroundColour(wx.SystemSettings.GetColour( wx.SYS_COLOUR_BTNTEXT ) )

self.hold_spin_21.SetBackgroundColour(wx.SystemSettings.GetColour( wx.SYS_COLOUR_BTNSHADOW ) )

POC_sizer_21.Add( self.hold_spin_21,wx.GBPosition( 7,1 ), wx.GBSpan( 1,1 ),

wx.ALIGN_CENTER_HORIZONTAL|wx.RIGHT|wx.LEFT|wx.BOTTOM, 5 )

self.maxHold_text_21 =wx.StaticText( self.findPOC_panel_1, wx.ID_ANY, u"600", wx.DefaultPosition, wx.DefaultSize, 0 )

self.maxHold_text_21.Wrap( -1 )

self.maxHold_text_21.SetFont( wx.Font( 12, 74, 90, 90, False, "Calibri" ) )

self.maxHold_text_21.SetForegroundColour $((96,96,96))$

POC_sizer_21.Add( self.maxHold_text_21, wx.GBPosition( 7, 2 ), wx.GBSpan( 1,1 ),

wx.LEFT|wx.RIGHT|wx.ALIGN_RIGHT|wx.ALIGN_CENTER_VERTICAL, 5 )

self.radioButton_panel_21 = wx.Panel( self.findPOC_panel_1, wx.ID_ANY, wx.DefaultPosition, wx.DefaultSize, wx.TAB_TRAVERSAL )

self.radioButton_panel_21.SetBackgroundColour(wx.SystemSettings.GetColour(wx.SYS_COLOUR_HIGHLIGHTTEXT ))

radioButton_sizer_21 $=$ wx.GridSizer( $0,3,0,0)$

self.cycle_21 = wx.RadioButton( self.radioButton_panel_21, wx.ID_ANY, u"Cycle", wx.DefaultPosition, wx.DefaultSize, 0 )

self.cycle_21.SetFont( wx.Font( 12, 74, 90, 90, False, "Calibri" ) )

self.cycle_21.SetValue(True)

radioButton_sizer_21.Add( self.cycle_21, 0, wx.ALL|wx.ALIGN_CENTER_HORIZONTAL, 5 )

self.retract_21 = wx.RadioButton( self.radioButton_panel_21, wx.ID_ANY, u"Retract", wx.DefaultPosition, wx.DefaultSize, 0 )

self.retract_21.SetFont( wx.Font( 12, 74, 90, 90, False, "Calibri" ) )

radioButton_sizer_21.Add( self.retract_21, 0, wx.ALL|wx.ALIGN_CENTER_HORIZONTAL, 5 )

self.extend_21 = wx.RadioButton( self.radioButton_panel_21, wx.ID_ANY, u"Extend", wx.DefaultPosition, wx.DefaultSize, 0 )

self.extend_21.SetFont( wx.Font( 12, 74, 90, 90, False, "Calibri" ))

radioButton_sizer_21.Add( self.extend_21,0, wx.ALL|wx.ALIGN_CENTER_HORIZONTAL, 5 )

self.radioButton_panel_21.SetSizer(radioButton_sizer_21 )

self.radioButton_panel_21.Layout() 
radioButton_sizer_21.Fit( self.radioButton_panel_21)

POC_sizer_21.Add( self.radioButton_panel_21, wx.GBPosition( 8, 0 ), wx.GBSpan( 1,3 ), wx.EXPAND |wx.ALL, 5 )

findPOC_sizer_1.Add( POC_sizer_21, 0, wx.ALIGN_CENTER_VERTICAL|wx.ALIGN_CENTER_HORIZONTAL, 5 )

button_poc_sizer $=$ wx.BoxSizer $($ wx.VERTICAL $)$

self.indent_poc_1 $=$ wx.Button( self.findPOC_panel_1, 12, u"Indent", wx.DefaultPosition, wx.Size( 175,60 ), 0 )

self.indent_poc_1.SetFont( wx.Font( 18, 74, 90, 92, False, "Calibri" ) )

self.Bind(wx.EVT_BUTTON,self.displacementIndent,id=12)

self.indent_poc_1.Disable()

self.indent_poc_1.SetMinSize( wx.Size (175,60 ) )

button_poc_sizer.Add( self.indent_poc_1, 0, wx.ALIGN_CENTER_HORIZONTAL|wx.TOP|wx.BOTTOM, 5 )

findPOC_sizer_1.Add( button_poc_sizer, 1, wx.EXPAND, 5 )

self.findPOC_panel_1.SetSizer(findPOC_sizer_1)

self.findPOC_panel_1.Layout()

step1_sizer.Add( self.findPOC_panel_1, 1, wx.EXPAND, 5 )

self.setPOC_panel_1 = wx.Panel( self.step1_panel, wx.ID_ANY, wx.DefaultPosition, wx.Size( 660,140 ), wx.SIMPLE_BORDER )

setPOC_sizer_1 $=$ wx.BoxSizer $($ wx. VERTICAL $)$

setPOC_sizer_1.SetMinSize( wx.Size $(-1,250)$ )

setPOC_text_sizer_1 = wx.FlexGridSizer( $3,1,0,0)$

setPOC_text_sizer_1.SetFlexibleDirection( wx.BOTH )

setPOC_text_sizer_1.SetNonFlexibleGrowMode( wx.FLEX_GROWMODE_SPECIFIED)

self.setPOC_text_1 = wx.StaticText( self.setPOC_panel_1, wx.ID_ANY, u"Set first point of contact", wx.DefaultPosition, wx.DefaultSize,

wx.ALIGN_LEFT )

self.setPOC_text_1.Wrap( -1 )

self.setPOC_text_1.SetFont( wx.Font (14, 74, 90, 92, False, "Calibri" ) )

self.setPOC_text_1.SetMinSize(wx.Size( 200,-1))

setPOC_text_sizer_1.Add( self.setPOC_text_1, 1, wx.TOP|wx.BOTTOM|wx.LEFT, 5 )

setPOC_sizer_1.Add( setPOC_text_sizer_1, 1, 0,5)

setPOC_button_sizer_1 =wx.FlexGridSizer $(3,0,0,0)$

setPOC_button_sizer_1.SetFlexibleDirection(wx.BOTH )

setPOC_button_sizer_1.SetNonFlexibleGrowMode( wx.FLEX_GROWMODE_SPECIFIED )

self.setPOC_button_1 = wx.Button( self.setPOC_panel_1, 13, u"Set", wx.DefaultPosition, wx.Size( 175,60 ), 0 )

self.setPOC_button_1.SetFont( wx.Font( 18, 74, 90, 92, False, "Calibri" ))

self.Bind(wx.EVT_BUTTON,self.setPointOfContact1,id=13)

self.setPOC_button_1.Disable()

setPOC_button_sizer_1.Add( self.setPOC_button_1, 0, wx.ALIGN_CENTER_HORIZONTAL|wx.TOP|wx.BOTTOM, 5 )

setPOC_sizer_1.Add( $\operatorname{setPOC}$ button_sizer_1, 0, wx.ALIGN_CENTER_HORIZONTAL, 5 )

self.setPOC_panel_1.SetSizer(setPOC_sizer_1 )

self.setPOC_panel_1.Layout()

step1_sizer.Add( self.setPOC_panel_1, 1, wx.EXPAND, 5 )

self.step1_panel.SetSizer( step1_sizer)

self.step1_panel.Layout()

step1_sizer.Fit(self.step1_panel )

decisionSizer.Add( self.step1_panel, 1, wx.EXPAND, 5 )

self.step2_panel $=$ wx.Panel $($ self.decisionPanel, wx.ID_ANY, wx.DefaultPosition, wx.DefaultSize, wx.TAB_TRAVERSAL )

self.step2_panel.Enable( False )

self.step2_panel.Hide()

step2_sizer $=$ wx.FlexGridSizer $(3,1,0,0)$

step2_sizer.SetFlexibleDirection( wx.VERTICAL )

step2_sizer.SetNonFlexibleGrowMode( wx.FLEX_GROWMODE_SPECIFIED )

step2_sizer.SetMinSize( wx.Size (660,600) )

self.findPOC_panel_2 = wx.Panel( self.step2_panel, wx.ID_ANY, wx.DefaultPosition, wx.Size( 660,400), wx.SIMPLE_BORDER )

findPOC_sizer_2 $=$ wx.FlexGridSizer $(3,1,0,0)$

findPOC_sizer_2.SetFlexibleDirection(wx.BOTH)

findPOC_sizer_2.SetNonFlexibleGrowMode(wx.FLEX_GROWMODE_SPECIFIED )

panel_header_sizer_2 $=$ wx.FlexGridSizer $(0,2,0,0)$

panel_header_sizer_2.SetFlexibleDirection(wx.HORIZONTAL )

panel_header_sizer_2.SetNonFlexibleGrowMode(wx.FLEX_GROWMODE_SPECIFIED )

panel_header_sizer_2.SetMinSize( wx.Size $(660,-1)$ )

self.findPOC_text $2=$ wx.StaticText( self.findPOC_panel_2, wx.ID_ANY, u"Indentation parameters", wx.DefaultPosition, wx.DefaultSize, 0 )

self.findPOC_text2.Wrap( -1$)$

self.findPOC_text2.SetFont( wx.Font( 14, 74, 90, 92, False, "Calibri" ) )

panel_header_sizer_2.Add( self.findPOC_text2, 0, wx.ALL, 5 )

findPOC_sizer_2.Add( panel_header_sizer_2, 1, wx.EXPAND, 5 )

POC_sizer_2 $=$ wx.GridBagSizer $(0,0)$

POC_sizer_2.SetFlexibleDirection( wx.BOTH)

POC_sizer_2.SetNonFlexibleGrowMode( wx.FLEX_GROWMODE_SPECIFIED )

self.max_text_2 =wx.StaticText( self.findPOC_panel_2, wx.ID_ANY, u"Max", wx.DefaultPosition, wx.DefaultSize, 0 ) 
self.max_text_2.Wrap(-1)

self.max_text_2.SetFont(wx.Font( 12, 74, 90, 90, False, "Calibri" ) )

self.max_text_2.SetForegroundColour( wx.SystemSettings.GetColour(wx.SYS_COLOUR_SCROLLBAR ) )

POC_sizer_2.Add( self.max_text_2, wx.GBPosition( 0,2),wx.GBSpan( 1,1 ),

wx.ALIGN_RIGHT|wx.ALIGN_CENTER_VERTICAL|wx.RIGHT|wx.TOP|wx.BOTTOM, 5 )

self.displacement_text_2 =wx.StaticText( self.findPOC_panel_2, wx.ID_ANY, u"displ. (mm)", wx.DefaultPosition, wx.DefaultSize,

wx.ALIGN_LEFT )

self.displacement_text_2.Wrap( -1 )

self.displacement_text_2.SetFont( wx.Font( 12, 74, 90, 90, False, "Calibri" ) )

POC_sizer_2.Add( self.displacement_text_2, wx.GBPosition( 0, 1 ), wx.GBSpan( 1, 1), wx.LEFT|wx.RIGHT|wx.ALIGN_BOTTOM, 5 ) self.min_text_2 = wx.StaticText( self.findPOC_panel_2, wx.ID_ANY, u"Min", wx.DefaultPosition, wx.DefaultSize, wx.ALIGN_LEFT ) self.min_text_2.Wrap (-1)

self.min_text_2.SetFont(wx.Font( 12, 74, 90, 90, False, "Calibri" ) )

self.min_text_2.SetForegroundColour(wx.SystemSettings.GetColour(wx.SYS_COLOUR_SCROLLBAR ) )

POC_sizer_2.Add( self.min_text_2, wx.GBPosition $(0,0)$, wx.GBSpan $(1,1)$,

wx.RIGHT|wx.TOP|wx.BOTTOM|wx.ALIGN_LEFT|wx.ALIGN_CENTER_VERTICAL, 5 )

self.minDisp_text_2 = wx.StaticText( self.findPOC_panel_2, wx.ID_ANY, u"0.01", wx.DefaultPosition, wx.Size ( $-1,-1$ ), wx.ALIGN_LEFT )

self.minDisp_text_2.Wrap ( -1 )

self.minDisp_text_2.SetFont( wx.Font( $12,74,90,90$, False, "Calibri" ) )

self.minDisp_text_2.SetForegroundColour( wx.SystemSettings.GetColour( wx.SYS_COLOUR_SCROLLBAR ) )

self.minDisp_text_2.SetMinSize( wx.Size (100,-1 ) )

POC_sizer_2.Add( self.minDisp_text_2, wx.GBPosition( 1,0 ), wx.GBSpan( 1,1 ),

wx.ALIGN_LEFT|wx.TOP|wx.BOTTOM|wx.ALIGN_CENTER_VERTICAL, 5 )

self.disp_spin_2 $=$ FS.FloatSpin( $\operatorname{self}$. findPOC_panel_2, -1, value $=.6, \min \_v a l=.01, \max \_v a l=5$,increment $=.1$, agwStyle=FS.FS_RIGHT )

self.disp_spin_2.SetFormat("\%f")

self.disp_spin_2.SetDigits(2)

self.disp_spin_2.SetFont( wx.Font( 12, 74, 90, 90, False, "Calibri" ) )

self.disp_spin_2.SetForegroundColour(wx.SystemSettings.GetColour(wx.SYS_COLOUR_BTNTEXT ) )

self.disp_spin_2.SetBackgroundColour( wx.SystemSettings.GetColour( wx.SYS_COLOUR_BTNSHADOW ) )

POC_sizer_2.Add( self.disp_spin_2, wx.GBPosition( 1,1$)$, wx.GBSpan $(1,1)$,

wx.BOTTOM|wx.RIGHT|wx.LEFT|wx.ALIGN_CENTER_HORIZONTAL, 5 )

self.minVel_text_2 = wx.StaticText( self.findPOC_panel_2, wx.ID_ANY, u"1.0", wx.DefaultPosition, wx.Size( $-1,-1$ ), wx.ALIGN_LEFT )

self.minVel_text_2.Wrap( -1 )

self.minVel_text_2.SetFont(wx.Font( 12, 74, 90, 90, False, "Calibri" ) )

self.minVel_text_2.SetForegroundColour(wx.SystemSettings.GetColour(wx.SYS_COLOUR_SCROLLBAR ) )

POC_sizer_2.Add( self.minVel_text_2, wx.GBPosition( 3,0 ), wx.GBSpan( 1, 1),

wx.ALIGN_LEFT|wx.TOP|wx.BOTTOM|wx.RIGHT|wx.ALIGN_CENTER_VERTICAL, 5 )

self.max_dispText_2 = wx.StaticText( self.findPOC_panel_2, wx.ID_ANY, u"5", wx.DefaultPosition, wx.DefaultSize, wx.ALIGN_RIGHT )

self.max_dispText_2.Wrap( -1 )

self.max_dispText_2.SetFont( wx.Font( 12, 74, 90, 90, False, "Calibri" ) )

self.max_dispText_2.SetForegroundColour(wx.SystemSettings.GetColour( wx.SYS_COLOUR_SCROLLBAR ) )

self.max_dispText_2.SetMinSize(wx.Size (100,-1 ))

POC_sizer_2.Add( self.max_dispText_2, wx.GBPosition( 1,2), wx.GBSpan( 1, 1 ),

wx.ALL/wx.ALIGN_CENTER_HORIZONTAL|wx.ALIGN_CENTER_VERTICAL, 5 )

self.vel_text_2 $=$ wx.StaticText( self.findPOC_panel_2, wx.ID_ANY, u"velocity $(\mathrm{mm} / \mathrm{s})$ ", wx.DefaultPosition, wx.DefaultSize,

wx.ALIGN_LEFT )

self.vel_text_2.Wrap ( -1)

self.vel_text_2.SetFont( wx.Font( 12, 74, 90, 90, False, "Calibri" ) )

POC_sizer_2.Add( self.vel_text_2, wx.GBPosition( 2,1 ), wx.GBSpan( 1,1 ), wx.ALIGN_BOTTOM|wx.LEFT|wx.RIGHT|wx.TOP, 5 )

self.vel_spin_2 $=$ FS.FloatSpin( self.findPOC_panel_2, -1 , value $=1, \min \_v a l=1, \max \_v a l=40.0$, increment $=.01$, agwStyle $=$ FS.FS_RIGHT )

self.vel_spin_2.SetFormat("\%f")

self.vel_spin_2.SetDigits(2)

self.vel_spin_2.SetFont( wx.Font( 12, 74, 90, 90, False, "Calibri" ) )

self.vel_spin_2.SetForegroundColour( wx.SystemSettings.GetColour( wx.SYS_COLOUR_BTNTEXT ))

self.vel_spin_2.SetBackgroundColour(wx.SystemSettings.GetColour(wx.SYS_COLOUR_BTNSHADOW ) )

POC_sizer_2.Add( self.vel_spin_2, wx.GBPosition( 3,1 ), wx.GBSpan( 1,1 ),

wx.BOTTOM|wx.RIGHT|wx.LEFT|wx.ALIGN_CENTER_HORIZONTAL, 5 )

self.maxVel_text_2 = wx.StaticText( self.findPOC_panel_2, wx.ID_ANY, u"40", wx.DefaultPosition, wx.DefaultSize, 0 )

self.maxVel_text_2.Wrap( -1 )

self.maxVel_text_2.SetFont( wx.Font( 12, 74, 90, 90, False, "Calibri" ) )

self.maxVel_text_2.SetForegroundColour(wx.SystemSettings.GetColour(wx.SYS_COLOUR_SCROLLBAR ) )

POC_sizer_2.Add( self.maxVel_text_2, wx.GBPosition( 3,2), wx.GBSpan( 1,1 ),

wx.ALL|wx.ALIGN_RIGHT|wx.ALIGN_CENTER_VERTICAL, 5 )

self.acc_text_2 $=$ wx.StaticText( self.findPOC_panel_2, wx.ID_ANY, u"accel. $(\mathrm{mm} / \mathrm{s} / \mathrm{s}) "$, wx.DefaultPosition, wx.DefaultSize,

wx.ALIGN_CENTRE )

self.acc_text_2.Wrap $(-1)$

self.acc_text_2.SetFont( wx.Font( 12, 74, 90, 90, False, "Calibri" ) ) 
POC_sizer_2.Add( self.acc_text_2, wx.GBPosition( 4,1 ), wx.GBSpan( 1,1 ), wx.TOP|wx.RIGHT|wx.LEFT, 5 )

self.min_Acc_text_2 =wx.StaticText( self.findPOC_panel_2, wx.ID_ANY, u"1.0", wx.DefaultPosition, wx.DefaultSize, 0 )

self.min_Acc_text_2.Wrap( -1 )

self.min_Acc_text_2.SetFont( wx.Font( 12, 74, 90, 90, False, "Calibri" ) )

self.min_Acc_text_2.SetForegroundColour(wx.SystemSettings.GetColour(wx.SYS_COLOUR_SCROLLBAR ) )

POC_sizer_2.Add( self.min_Acc_text_2, wx.GBPosition( 5, 0 ), wx.GBSpan ( 1,1 ),

wx.ALIGN_LEFT|wx.TOP|wx.BOTTOM|wx.RIGHT|wx.ALIGN_CENTER_VERTICAL, 5 )

self.acc_spin_2 $=$ FS.FloatSpin( self.findPOC_panel_2, -1 ,value $=40, \min \_$val $=.1$, max_val=40.0,increment $=.5$, agwStyle=FS.FS_RIGHT )

self.acc_spin_2.SetFormat("\%f")

self.acc_spin_2.SetDigits(2)

self.acc_spin_2.SetFont( wx.Font( 12, 74, 90, 90, False, "Calibri" ) )

self.acc_spin_2.SetForegroundColour(wx.SystemSettings.GetColour( wx.SYS_COLOUR_BTNTEXT ) )

self.acc_spin_2.SetBackgroundColour(wx.SystemSettings.GetColour( wx.SYS_COLOUR_BTNSHADOW ) )

POC_sizer_2.Add( self.acc_spin_2, wx.GBPosition $(5,1)$, wx.GBSpan $(1,1$ ),

wx.BOTTOM|wx.RIGHT|wx.LEFT|wx.ALIGN_CENTER_HORIZONTAL, 5 )

self.max_Acc_text_2 $=$ wx.StaticText( self.findPOC_panel_2, wx.ID_ANY, u"160", wx.DefaultPosition, wx.DefaultSize, 0 )

self.max_Acc_text_2.Wrap( -1 )

self.max_Acc_text_2.SetFont( wx.Font( 12, 74, 90, 90, False, "Calibri" ) )

self.max_Acc_text_2.SetForegroundColour(wx.SystemSettings.GetColour(wx.SYS_COLOUR_SCROLLBAR ) )

POC_sizer_2.Add( self.max_Acc_text_2, wx.GBPosition( 5,2), wx.GBSpan( 1,1 ),

wx.ALL|wx.ALIGN_RIGHT|wx.ALIGN_CENTER_VERTICAL, 5 )

self.hold_text_2 $=$ wx.StaticText( self.findPOC_panel_2, wx.ID_ANY, u"hold (s) ", wx.DefaultPosition, wx.DefaultSize, wx.ALIGN_CENTRE ) self.hold_text_2.Wrap( -1 )

self.hold_text_2.SetFont( wx.Font( 12, 74, 90, 90, False, "Calibri" ) )

POC_sizer_2.Add( self.hold_text_2, wx.GBPosition( 6, 1), wx.GBSpan( 1,1 ),

wx.TOP|wx.RIGHT|wx.LEFT|wx.ALIGN_CENTER_VERTICAL|wx.ALIGN_LEFT, 5 )

self.min_holdText_2 $=$ wx.StaticText( self.findPOC_panel_2, wx.ID_ANY, u"0.8", wx.DefaultPosition, wx.DefaultSize, wx.ALIGN_CENTRE )

self.min_holdText_2.Wrap( -1 )

self.min_holdText_2.SetFont( wx.Font( 12, 74, 90, 90, False, "Calibri" ) )

self.min_holdText_2.SetForegroundColour( wx.SystemSettings.GetColour(wx.SYS_COLOUR_SCROLLBAR ) )

POC_sizer_2.Add( self.min_holdText_2, wx.GBPosition( 7, 0 ), wx.GBSpan( 1,1 ),

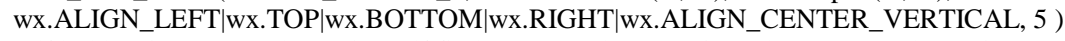

self.hold_spin_2 $=$ FS.FloatSpin( self.findPOC_panel_2, -1, min_val $=.1$, max_val=600,increment $=.5$, agwStyle=FS.FS_RIGHT )

self.hold_spin_2.SetFormat("\%f")

self.hold_spin_2.SetDigits(2)

self.hold_spin_2.SetFont( wx.Font( 12, 74, 90, 90, False, "Calibri" ) )

self.hold_spin_2.SetForegroundColour(wx.SystemSettings.GetColour(wx.SYS_COLOUR_BTNTEXT ) )

self.hold_spin_2.SetBackgroundColour(wx.SystemSettings.GetColour(wx.SYS_COLOUR_BTNSHADOW ) )

POC_sizer_2.Add( self.hold_spin_2, wx.GBPosition( 7,1),wx.GBSpan( 1,1 ),

wx.BOTTOM|wx.RIGHT|wx.LEFT|wx.ALIGN_CENTER_HORIZONTAL, 5 )

self.maxHold_text_2 $=$ wx.StaticText( self.findPOC_panel_2, wx.ID_ANY, u"600", wx.DefaultPosition, wx.DefaultSize, 0 )

self.maxHold_text_2.Wrap( -1 )

self.maxHold_text_2.SetFont( wx.Font( 12, 74, 90, 90, False, "Calibri" ) )

self.maxHold_text_2.SetForegroundColour(wx.SystemSettings.GetColour( wx.SYS_COLOUR_SCROLLBAR ) )

POC_sizer_2.Add( self.maxHold_text_2, wx.GBPosition( 7,2 ), wx.GBSpan( 1,1 ),

wx.ALL|wx.ALIGN_RIGHT|wx.ALIGN_CENTER_VERTICAL, 5 )

self.radioButton_panel_2 = wx.Panel( self.findPOC_panel_2, wx.ID_ANY, wx.DefaultPosition, wx.DefaultSize, wx.TAB_TRAVERSAL )

self.radioButton_panel_2.SetBackgroundColour(wx.SystemSettings.GetColour(wx.SYS_COLOUR_HIGHLIGHTTEXT ) )

radioButton_sizer_2 $=$ wx.GridSizer $(0,3,0,0)$

self.cycle_2 = wx.RadioButton( self.radioButton_panel_2, wx.ID_ANY, u"Cycle", wx.DefaultPosition, wx.DefaultSize, 0 )

self.cycle_2.SetFont( wx.Font( 12, 74, 90, 90, False, "Calibri" ) )

self.cycle_2.SetValue(True)

radioButton_sizer_2.Add( self.cycle_2, 0, wx.ALL|wx.ALIGN_CENTER_HORIZONTAL, 5 )

self.retract_2 $=$ wx.RadioButton( self.radioButton_panel_2, wx.ID_ANY, u"Retract", wx.DefaultPosition, wx.DefaultSize, 0 )

self.retract_2.SetFont( wx.Font ( 12, 74, 90, 90, False, "Calibri" ) )

radioButton_sizer_2.Add( self.retract_2, 0, wx.ALL|wx.ALIGN_CENTER_HORIZONTAL, 5 )

self.extend_2 = wx.RadioButton( self.radioButton_panel_2, wx.ID_ANY, u"Extend", wx.DefaultPosition, wx.DefaultSize, 0 )

self.extend_2.SetFont( wx.Font (12, 74, 90, 90, False, "Calibri" ) )

radioButton_sizer_2.Add( self.extend_2, 0, wx.ALL|wx.ALIGN_CENTER_HORIZONTAL, 5 )

self.radioButton_panel_2.SetSizer( radioButton_sizer_2 )

self.radioButton_panel_2.Layout()

radioButton_sizer_2.Fit( self.radioButton_panel_2)

POC_sizer_2.Add( self.radioButton_panel_2, wx.GBPosition( 8, 0 ), wx.GBSpan( 1,3 ), wx.EXPAND |wx.ALL, 5 )

findPOC_sizer_2.Add( POC_sizer_2, 1, wx.ALIGN_CENTER_HORIZONTAL|wx.ALIGN_CENTER_VERTICAL, 5 )

indentButton_sizer_2 $=$ wx.BoxSizer( wx.VERTICAL )

self.indent_Button_2 = wx.Button( self.findPOC_panel_2, 14, u"Indent", wx.DefaultPosition, wx.Size( 175,60 ), 0 )

self.indent_Button_2.SetFont(wx.Font( 18, 74, 90, 92, False, "Calibri" ))

self.Bind(wx.EVT_BUTTON,self.displacementIndent2,id=14) 
indentButton_sizer_2.Add( self.indent_Button_2, 0, wx.ALIGN_CENTER_HORIZONTAL|wx.ALL, 5 )

findPOC_sizer_2.Add( indentButton_sizer_2, 1 , wx.EXPAND, 5 )

self.findPOC_panel_2.SetSizer(findPOC_sizer_2 )

self.findPOC_panel_2.Layout()

step2_sizer.Add( self.findPOC_panel_2, 1, wx.EXPAND, 5 )

self.DispMin_DispMax_panel = wx.Panel( self.step2_panel, wx.ID_ANY, wx.DefaultPosition, wx.Size (660,-1 ), wx.SIMPLE_BORDER )

dispDisplay_sizer_2 $=$ wx.BoxSizer( wx.HORIZONTAL )

dispDisplay_sizer_2.SetMinSize( wx.Size $(-1,250)$ )

dispMin_sizer_2 $=$ wx.FlexGridSizer $(3,1,0,0)$

dispMin_sizer_2.SetFlexibleDirection( wx.BOTH )

dispMin_sizer_2.SetNonFlexibleGrowMode( wx.FLEX_GROWMODE_SPECIFIED )

self.Disp_min_text_2 = wx.StaticText( self.DispMin_DispMax_panel, wx.ID_ANY, u"Displacement Minimum", wx.DefaultPosition, wx.DefaultSize, wx.ALIGN CENTRE )

self.Disp_min_text_2.Wrap( -1 )

self.Disp_min_text_2.SetFont( wx.Font( 14, 74, 90, 92, False, "Calibri" ) )

self.Disp_min_text_2.SetMinSize(wx.Size $(330,-1)$ )

dispMin_sizer_2.Add( self.Disp_min_text_2, 1, wx.TOP|wx.BOTTOM, 5 )

self.displaySizer_2 $=$ wx.FlexGridSizer $(0,2,0,0)$

self.displaySizer_2.SetFlexibleDirection(wx.BOTH )

self.displaySizer_2.SetNonFlexibleGrowMode( wx.FLEX_GROWMODE_SPECIFIED )

self.dispMin_text_2 = wx.StaticText( self.DispMin_DispMax_panel, wx.ID_ANY, u"Disp (mm.)", wx.DefaultPosition, wx.DefaultSize, 0 )

self.dispMin_text_2.Wrap( -1 )

self.dispMin_text_2.SetFont( wx.Font( 12, 74, 90, 90, False, "Calibri" ) )

self.displaySizer_2.Add( self.dispMin_text_2, 0, wx.ALL|wx.ALIGN_CENTER_HORIZONTAL, 5 )

self.forceMin_text_2 $=$ wx.StaticText( self.DispMin_DispMax_panel, wx.ID_ANY, u"Force (mN)", wx.DefaultPosition, wx.DefaultSize, 0 ) self.forceMin_text_2.Wrap( -1$)$

self.forceMin_text_2.SetFont( wx.Font( 12, 74, 90, 90, False, "Calibri" ) )

self.displaySizer_2.Add( self.forceMin_text_2, 0, wx.ALL/wx.ALIGN_CENTER_HORIZONTAL, 5 )

displayMinDisp_box_2 = wx.TextCtrl( self.DispMin_DispMax_panel, wx.ID_ANY, wx.EmptyString, wx.DefaultPosition, wx.DefaultSize, 0 ) displayMinDisp_box_2.SetFont( wx.Font( 14, 74, 90, 90, False, "Calibri" ) )

self.displaySizer_2.Add( displayMinDisp_box_2, 0, wx.BOTTOM|wx.RIGHT|wx.LEFT, 5 )

displayMinForce_box_2 =wx.TextCtrl( self.DispMin_DispMax_panel, wx.ID_ANY, wx.EmptyString, wx.DefaultPosition, wx.DefaultSize, 0 )

displayMinForce_box_2.SetFont( wx.Font( 14, 74, 90, 90, False, "Calibri" ) )

self.displaySizer_2.Add(displayMinForce_box_2, 0, wx.BOTTOM|wx.RIGHT|wx.LEFT, 5 )

dispMin_sizer_2.Add( self.displaySizer_2, 1, wx.ALIGN_CENTER_HORIZONTAL, 5 )

self.setMin_button_2 = wx.Button( self.DispMin_DispMax_panel, 15, u"Set Min", wx.DefaultPosition, wx.Size( 175,60 ), 0 )

self.setMin_button_2.SetFont( wx.Font( 18, 74, 90, 92, False, "Calibri" ) )

self.Bind(wx.EVT_BUTTON,self.setDispMin,id=15)

dispMin_sizer_2.Add( self.setMin_button_2, 0, wx.ALL|wx.ALIGN_CENTER_HORIZONTAL, 5 )

dispDisplay_sizer_2.Add(dispMin_sizer_2, $1,0,5$ )

dispMax_sizer_2 = wx.FlexGridSizer $(3,0,0,0)$

dispMax_sizer_2.SetFlexibleDirection( wx.BOTH )

dispMax_sizer_2.SetNonFlexibleGrowMode( wx.FLEX_GROWMODE_SPECIFIED)

self.Disp_max_text_2 = wx.StaticText( self.DispMin_DispMax_panel, wx.ID_ANY, u"Displacement Maximum", wx.DefaultPosition,

wx.DefaultSize, wx.ALIGN_CENTRE )

self.Disp_max_text_2.Wrap( -1$)$

self.Disp_max_text_2.SetFont( wx.Font (14, 74, 90, 92, False, "Calibri" ) )

self.Disp_max_text_2.SetMinSize( wx.Size (330,-1 ))

dispMax_sizer_2.Add( self.Disp_max_text_2, 0, wx.TOP|wx.BOTTOM, 5 )

dispMax_sizer_2 $1=$ wx.FlexGridSizer $2,2,0,0)$

dispMax_sizer_2_1.SetFlexibleDirection(wx.BOTH )

dispMax_sizer_2_1.SetNonFlexibleGrowMode(wx.FLEX_GROWMODE_SPECIFIED)

self.dispMax_text_2 = wx.StaticText( self.DispMin_DispMax_panel, wx.ID_ANY, u"Displ. (mm)", wx.DefaultPosition, wx.DefaultSize, 0 )

self.dispMax_text_2.Wrap( -1 )

self.dispMax_text_2.SetFont(wx.Font( 12, 74, 90, 90, False, "Calibri" ) )

dispMax_sizer_2_1.Add( self.dispMax_text_2, 0, wx.ALL|wx.ALIGN_CENTER_HORIZONTAL, 5 )

self.forceMax_text_2 = wx.StaticText( self.DispMin_DispMax_panel, wx.ID_ANY, u"Force (mN)", wx.DefaultPosition, wx.DefaultSize, 0 )

self.forceMax_text_2.Wrap (-1)

self.forceMax_text_2.SetFont( wx.Font( 12, 74, 90, 90, False, "Calibri" ) )

dispMax_sizer_2_1.Add( self.forceMax_text_2, 0, wx.ALL|wx.ALIGN_CENTER_HORIZONTAL, 5 )

dispMaxDisp_box_2 $=$ wx.TextCtrl( self.DispMin_DispMax_panel, wx.ID_ANY, wx.EmptyString, wx.DefaultPosition, wx.DefaultSize, 0 )

dispMaxDisp_box_2.SetFont( wx.Font( 14, 74, 90, 90, False, "Calibri" ) )

dispMax_sizer_2_1.Add( dispMaxDisp_box_2, 0, wx.BOTTOM|wx.RIGHT|wx.LEFT, 5 )

dispMaxForce_box_2 = wx.TextCtrl( self.DispMin_DispMax_panel, wx.ID_ANY, wx.EmptyString, wx.DefaultPosition, wx.DefaultSize, 0 )

dispMaxForce_box_2.SetFont( wx.Font( 14, 74, 90, 90, False, "Calibri" ) )

dispMax_sizer_2_1.Add( dispMaxForce_box_2, 0, wx.BOTTOM|wx.RIGHT|wx.LEFT, 5 )

dispMax_sizer_2.Add(dispMax_sizer_2_1, 0, wx.ALIGN_CENTER_HORIZONTAL, 5 )

self.setMax_button_2 = wx.Button( self.DispMin_DispMax_panel, 16, u"Set Max", wx.DefaultPosition, wx.Size (175,60 ), 0 ) 
self.setMax_button_2.SetFont( wx.Font( 18, 74, 90, 92, False, "Calibri" ) )

self.Bind(wx.EVT_BUTTON,self.setDispMax,id=16)

dispMax_sizer_2.Add( self.setMax_button_2, 0, wx.ALL|wx.ALIGN_CENTER_HORIZONTAL, 5 )

dispDisplay_sizer_2.Add( dispMax_sizer_2, 1 , wx.EXPAND, 5 )

self.DispMin_DispMax_panel.SetSizer(dispDisplay_sizer_2 )

self.DispMin_DispMax_panel.Layout()

step2_sizer.Add( self.DispMin_DispMax_panel, 1, wx.EXPAND, 5 )

self.step2_panel.SetSizer( step2_sizer )

self.step2_panel.Layout()

step2_sizer.Fit( self.step2_panel )

decisionSizer.Add( self.step2_panel, 1, wx.EXPAND, 5 )

self.step3_panel $=$ wx.Panel( self.decisionPanel, wx.ID_ANY, wx.DefaultPosition, wx.DefaultSize, wx.TAB_TRAVERSAL )

self.step3_panel.Enable( False )

self.step3_panel.Hide()

step3_sizer $=$ wx.FlexGridSizer $(3,1,0,0)$

step3_sizer.SetFlexibleDirection(wx.VERTICAL )

step3_sizer.SetNonFlexibleGrowMode( wx.FLEX_GROWMODE_SPECIFIED )

step3_sizer.SetMinSize( wx.Size (660,600) )

self.pre_indent_panel_3 = wx.Panel( self.step3_panel, wx.ID_ANY, wx.DefaultPosition, wx.DefaultSize, wx.SIMPLE_BORDER )

pre_indent_sizer_3 $=$ wx.FlexGridSizer $(4,1,0,0)$

pre_indent_sizer_3.SetFlexibleDirection(wx.BOTH)

pre_indent_sizer_3.SetNonFlexibleGrowMode( wx.FLEX_GROWMODE_SPECIFIED )

pre_indent_text_sizer $=$ wx.FlexGridSizer $(0,3,0,0)$

pre_indent_text_sizer.SetFlexibleDirection(wx.BOTH )

pre_indent_text_sizer.SetNonFlexibleGrowMode( wx.FLEX_GROWMODE_SPECIFIED )

pre_indent_text_sizer.SetMinSize( wx.Size $(660,-1)$ )

self.pre_indent_text_3 = wx.StaticText( self.pre_indent_panel_3, wx.ID_ANY, u"Pre-indentation:", wx.DefaultPosition, wx.Size( $-1,25$ ), 0 )

self.pre_indent_text_3.Wrap( -1 )

self.pre_indent_text_3.SetFont( wx.Font( 14, 74, 90, 92, False, "Calibri" ) )

pre_indent_text_sizer.Add( self.pre_indent_text_3, 0, wx.ALL, 5 )

self.preindent_descr_3 = wx.StaticText( self.pre_indent_panel_3, wx.ID_ANY, u"This button initiates 3 max displacement stimuli in sequence", wx.DefaultPosition, wx.Size (475,25), 0 )

self.preindent_descr_3.Wrap( -1$)$

self.preindent_descr_3.SetFont( wx.Font( 14, 74, 90, 90, False, "Calibri" ) )

pre_indent_text_sizer.Add( self.preindent_descr_3, 0 , wx.ALL, 5 )

pre_indent_sizer_3.Add( pre_indent_text_sizer, 1, wx.EXPAND, 5 )

self.preindent_disp_3 $=$ wx.StaticText( self.pre_indent_panel_3, wx.ID_ANY, u"Pre-Indent Displacement", wx.DefaultPosition,wx.DefaultSize, 0 )

self.preindent_disp_3.Wrap( -1 )

self.preindent_disp_3.SetFont( wx.Font( 12, 74, 90, 90, False, "Calibri" ) )

pre_indent_sizer_3.Add( self.preindent_disp_3, 0, wx.ALL|wx.ALIGN_CENTER_HORIZONTAL, 5 )

self.highestDisp_3 = wx.TextCtrl( self.pre_indent_panel_3, wx.ID_ANY,u"0", wx.DefaultPosition, wx.DefaultSize, 0 )

self.highestDisp_3.SetFont( wx.Font( 14, 74, 90, 90, False, "Calibri" ) )

pre_indent_sizer_3.Add( self.highestDisp_3,0,wx.ALL|wx.ALIGN_CENTER_HORIZONTAL)\#, wx.GBPosition( 1,1 ), wx.GBSpan( 1,1 ),

wx.ALIGN_CENTER_HORIZONTAL|wx.RIGHT|wx.LEFT, 5 )

self.start_preindent_button_3 = wx.Button(self.pre_indent_panel_3, 17, u"Start", wx.DefaultPosition, wx.DefaultSize, 0 )

self.start_preindent_button_3.SetFont( wx.Font( 18, 74, 90, 92, False, "Calibri" ) )

self.start_preindent_button_3.SetMinSize(wx.Size (175,60) )

self.Bind(wx.EVT_BUTTON,self.preIndent,id=17)

pre_indent_sizer_3.Add( self.start_preindent_button_3, 0, wx.ALL|wx.ALIGN_CENTER_HORIZONTAL, 5)

self.pre_indent_panel_3.SetSizer(pre_indent_sizer_3 )

self.pre_indent_panel_3.Layout()

pre_indent_sizer_3.Fit( self.pre_indent_panel_3 )

step3_sizer.Add( self.pre_indent_panel_3, 1, wx.EXPAND, 5 )

self.findPOC_panel_3 =wx.Panel( self.step3_panel, wx.ID_ANY, wx.DefaultPosition, wx.Size( 660,350), wx.SIMPLE_BORDER )

findPOC_sizer_3 $=$ wx.FlexGridSizer $(3,1,0,0)$

findPOC_sizer_3.SetFlexibleDirection(wx.BOTH)

findPOC_sizer_3.SetNonFlexibleGrowMode( wx.FLEX_GROWMODE_SPECIFIED)

panel_header_sizer_3 $=$ wx.FlexGridSizer $(0,2,0,0)$

panel_header_sizer_3.SetFlexibleDirection(wx.HORIZONTAL )

panel_header_sizer_3.SetNonFlexibleGrowMode( wx.FLEX_GROWMODE_SPECIFIED )

panel_header_sizer_3.SetMinSize (wx.Size $(660,-1))$

self.findPOC_text_3 = wx.StaticText( self.findPOC_panel_3, wx.ID_ANY, u"Find point of contact:", wx.DefaultPosition, wx.DefaultSize, 0 )

self.findPOC_text_3.Wrap( -1$)$

self.findPOC_text_3.SetFont( wx.Font( 14, 74, 90, 92, False, "Calibri" ) )

panel_header_sizer_3.Add( self.findPOC_text_3, 0, 5 )

self.findPOC_text_descr_3 = wx.StaticText( self.findPOC_panel_3, wx.ID_ANY, u"Indentation parameters", wx.DefaultPosition,

wx.DefaultSize, 0 ) 
self.findPOC_text_descr_3.Wrap( -1 )

self.findPOC_text_descr_3.SetFont( wx.Font( 14, 74, 90, 90, False, "Calibri" ) )

self.findPOC_text_descr_3.SetMinSize( wx.Size $(450,-1)$ )

panel_header_sizer_3.Add( self.findPOC_text_descr_3, 0,5)

findPOC_sizer_3.Add( panel_header_sizer_3, 1, wx.EXPAND, 5 )

POC_sizer_3 $=$ wx.GridBagSizer $(0,0)$

POC_sizer_3.SetFlexibleDirection(wx.BOTH)

POC_sizer_3.SetNonFlexibleGrowMode(wx.FLEX_GROWMODE_SPECIFIED )

self.max_text_3 = wx.StaticText( self.findPOC_panel_3, wx.ID_ANY, u"Max", wx.DefaultPosition, wx.DefaultSize, 0 )

self.max_text_3.Wrap( -1$)$

self.max_text_3.SetFont( wx.Font( 12, 74, 90, 90, False, "Calibri" ) )

self.max_text_3.SetForegroundColour(wx.SystemSettings.GetColour( wx.SYS_COLOUR_SCROLLBAR ) )

POC_sizer_3.Add( self.max_text_3, wx.GBPosition( 0,2), wx.GBSpan( 1, 1 ),

wx.ALIGN_RIGHT|wx.ALIGN_CENTER_VERTICAL|wx.LEFT|wx.TOP|wx.BOTTOM, 5 )

self.displacement_text_3 =wx.StaticText( self.findPOC_panel_3, wx.ID_ANY, u"displ. (mm)",wx.DefaultPosition, wx.DefaultSize,

wx.ALIGN_CENTRE )

self.displacement_text_3.Wrap( -1 )

self.displacement_text_3.SetFont( wx.Font (12, 74, 90, 90, False, "Calibri" ) )

POC_sizer_3.Add( self.displacement_text_3, wx.GBPosition( 0, 1 ), wx.GBSpan $(1,1$ ), wx.LEFT|wx.RIGHT|wx.ALIGN_BOTTOM, 5 )

self.min_text_3 = wx.StaticText( self.findPOC_panel_3, wx.ID_ANY, u"Min", wx.DefaultPosition, wx.Size( 40,-1 ), wx.ALIGN_LEFT )

self.min_text_3.Wrap( -1$)$

self.min_text_3.SetFont( wx.Font( 12, 74, 90, 90, False, "Calibri" ) )

self.min_text_3.SetForegroundColour(wx.SystemSettings.GetColour(wx.SYS_COLOUR_SCROLLBAR ))

POC_sizer_3.Add( self.min_text_3, wx.GBPosition $(0,0)$, wx.GBSpan $(1,1)$,

wx.RIGHT|wx.ALIGN_LEFT|wx.ALIGN_CENTER_VERTICAL, 5 )

self.minDisp_text_3 = wx.StaticText( self.findPOC_panel_3, wx.ID_ANY, u"0.01", wx.DefaultPosition, wx.Size( -1,-1 ), wx.ALIGN_LEFT )

self.minDisp_text_3.Wrap ( -1$)$

self.minDisp_text_3.SetFont( wx.Font( 12, 74, 90, 90, False, "Calibri" ) )

self.minDisp_text_3.SetForegroundColour( wx.SystemSettings.GetColour( wx.SYS_COLOUR_SCROLLBAR ) )

self.minDisp_text_3.SetMinSize( wx.Size( 100,-1 ))

POC_sizer_3.Add( self.minDisp_text_3, wx.GBPosition( 1,0 ), wx.GBSpan( 1,1 ),

wx.ALIGN_LEFT|wx.ALIGN_CENTER_VERTICAL|wx.RIGHT, 5 )

self.disp_spin_3 = FS.FloatSpin ( self.findPOC_panel_3,-1,value $=.6, \min \_v a l=.01$, max_val=5,increment $=.01$, agwStyle=FS.FS_RIGHT )

self.disp_spin_3.SetFormat("\%f")

self.disp_spin_3.SetDigits(2)

self.disp_spin_3.SetFont( wx.Font( 12, 74, 90, 90, False, "Calibri" ) )

self.disp_spin_3.SetForegroundColour(wx.SystemSettings.GetColour( wx.SYS_COLOUR_BTNTEXT ) )

self.disp_spin_3.SetBackgroundColour( wx.SystemSettings.GetColour( wx.SYS_COLOUR_BTNSHADOW ) )

POC_sizer_3.Add( self.disp_spin_3, wx.GBPosition( 1,1 ), wx.GBSpan $(1,1)$,

wx.ALIGN_CENTER_HORIZONTAL|wx.LEFT|wx.RIGHT|wx.BOTTOM, 5 )

self.minVel_text_3 $=$ wx.StaticText( self.findPOC_panel_3, wx.ID_ANY, u"1.0", wx.DefaultPosition, wx.Size( $-1,-1$ ), wx.ALIGN_LEFT )

self.minVel_text_3.Wrap $(-1)$

self.minVel_text_3.SetFont(wx.Font( 12, 74, 90, 90, False, "Calibri" ) )

self.minVel_text_3.SetForegroundColour(wx.SystemSettings.GetColour(wx.SYS_COLOUR_SCROLLBAR ) )

POC_sizer_3.Add( self.minVel_text_3, wx.GBPosition( 3,0), wx.GBSpan $(1,1)$,

wx.ALIGN_LEFT|wx.RIGHT|wx.ALIGN_CENTER_VERTICAL, 5 )

self.max_dispText_3 = wx.StaticText( self.findPOC_panel_3, wx.ID_ANY, u"5", wx.DefaultPosition, wx.DefaultSize, wx.ALIGN_RIGHT )

self.max_dispText_3.Wrap( -1 )

self.max_dispText_3.SetFont( wx.Font( 12, 74, 90, 90, False, "Calibri" ) )

self.max_dispText_3.SetForegroundColour(wx.SystemSettings.GetColour( wx.SYS_COLOUR_SCROLLBAR ) )

self.max_dispText_3.SetMinSize( wx.Size $(100,-1))$

POC_sizer_3.Add( self.max_dispText_3, wx.GBPosition( 1,2 ), wx.GBSpan( 1, 1),

wx.LEFT|wx.ALIGN_RIGHT|wx.ALIGN_CENTER_VERTICAL, 5 )

self.vel_text_3 $=$ wx.StaticText( self.findPOC_panel_3, wx.ID_ANY, u"velocity $(\mathrm{mm} / \mathrm{s})$ ", wx.DefaultPosition, wx.DefaultSize,

wx.ALIGN_LEFT )

self.vel_text_3.Wrap $(-1)$

self.vel_text_3.SetFont( wx.Font( 12, 74, 90, 90, False, "Calibri" ) )

POC_sizer_3.Add( self.vel_text_3, wx.GBPosition( 2, 1), wx.GBSpan ( 1, 1 ), wx.ALIGN_LEFT|wx.LEFT|wx.RIGHT, 5 )

self.vel_spin_3 $=$ FS.FloatSpin( self.findPOC_panel_3, -1 ,value $=1, \min \_v a l=1, \max \_v a l=40.0$, increment $=.5$, agwStyle=FS.FS_RIGHT )

self.vel_spin_3.SetFormat("\%f")

self.vel_spin_3.SetDigits(2)

self.vel_spin_3.SetFont( wx.Font( 12, 74, 90, 90, False, "Calibri" ) )

self.vel_spin_3.SetForegroundColour( wx.SystemSettings.GetColour( wx.SYS_COLOUR_BTNTEXT ))

self.vel_spin_3.SetBackgroundColour(wx.SystemSettings.GetColour(wx.SYS_COLOUR_BTNSHADOW ) )

POC_sizer_3.Add( self.vel_spin_3, wx.GBPosition( 3,1 ), wx.GBSpan( 1,1 ),

wx.ALIGN_CENTER_HORIZONTAL|wx.LEFT|wx.RIGHT|wx.BOTTOM, 5 )

self.maxVel_text_3 = wx.StaticText( self.findPOC_panel_3, wx.ID_ANY, u"40", wx.DefaultPosition, wx.DefaultSize, 0 )

self.maxVel_text_3.Wrap( -1 ) 
self.maxVel_text_3.SetFont( wx.Font( 12, 74, 90, 90, False, "Calibri" ) )

self.maxVel_text_3.SetForegroundColour(wx.SystemSettings.GetColour( wx.SYS_COLOUR_SCROLLBAR ) )

POC_sizer_3.Add( self.maxVel_text_3, wx.GBPosition( 3,2), wx.GBSpan( 1, 1 ),

wx.LEFT|wx.ALIGN_RIGHT|wx.ALIGN_CENTER_VERTICAL, 5 )

self.acc_text_3 = wx.StaticText( self.findPOC_panel_3, wx.ID_ANY, u"accel. (mm/s/s)", wx.DefaultPosition, wx.DefaultSize,

wx.ALIGN_CENTRE )

self.acc_text_3.Wrap $(-1)$

self.acc_text_3.SetFont( wx.Font( 12, 74, 90, 90, False, "Calibri" ) )

POC_sizer_3.Add( self.acc_text_3, wx.GBPosition( 4, 1), wx.GBSpan( 1, 1 ),wx.RIGHT|wx.LEFT, 5 )

self.min_Acc_text_3 $=$ wx.StaticText( self.findPOC_panel_3, wx.ID_ANY, u"1.0", wx.DefaultPosition, wx.DefaultSize, 0 )

self.min_Acc_text_3.Wrap( -1 )

self.min_Acc_text_3.SetFont(wx.Font( 12, 74, 90, 90, False, "Calibri" ) )

self.min_Acc_text_3.SetForegroundColour(wx.SystemSettings.GetColour(wx.SYS_COLOUR_SCROLLBAR ) )

POC_sizer_3.Add( self.min_Acc_text_3, wx.GBPosition( 5, 0), wx.GBSpan( 1,1 ),

wx.ALIGN_CENTER_VERTICAL/wx.RIGHT|wx.ALIGN_LEFT, 5 )

self.acc_spin_3 $=$ FS.FloatSpin $\left(\right.$ self.findPOC_panel_3, -1 ,value $=40, \min \_v a l=1$, max_val=160,increment $=1$, agwStyle=FS.FS_RIGHT )

self.acc_spin_3.SetFormat("\%f")

self.acc_spin_3.SetDigits(2)

self.acc_spin_3.SetFont( wx.Font( 12, 74, 90, 90, False, "Calibri" ) )

self.acc_spin_3.SetForegroundColour( wx.SystemSettings.GetColour( wx.SYS_COLOUR_BTNTEXT) )

self.acc_spin_3.SetBackgroundColour(wx.SystemSettings.GetColour(wx.SYS_COLOUR_BTNSHADOW ) )

POC_sizer_3.Add( self.acc_spin_3, wx.GBPosition $(5,1)$, wx.GBSpan $(1,1)$,

wx.ALIGN_CENTER_HORIZONTAL/wx.LEFT|wx.RIGHT|wx.BOTTOM, 5 )

self.max_Acc_text_3 = wx.StaticText( self.findPOC_panel_3, wx.ID_ANY, u"160", wx.DefaultPosition, wx.DefaultSize, 0 )

self.max_Acc_text_3.Wrap( -1 )

self.max_Acc_text_3.SetFont( wx.Font( 12, 74, 90, 90, False, "Calibri" ) )

self.max_Acc_text_3.SetForegroundColour(wx.SystemSettings.GetColour( wx.SYS_COLOUR_SCROLLBAR ) )

POC_sizer_3.Add( self.max_Acc_text_3, wx.GBPosition( 5, 2), wx.GBSpan( 1,1 ),

wx.LEFT|wx.ALIGN_CENTER_VERTICAL|wx.ALIGN_RIGHT, 5 )

self.hold_text_3 =wx.StaticText( self.findPOC_panel_3, wx.ID_ANY, u"hold (s) ", wx.DefaultPosition, wx.DefaultSize, wx.ALIGN_CENTRE )

self.hold_text_3.Wrap( -1 )

self.hold_text_3.SetFont( wx.Font( 12, 74, 90, 90, False, "Calibri" ) )

POC_sizer_3.Add( self.hold_text_3, wx.GBPosition( 6,1 ), wx.GBSpan ( 1,1

),wx.RIGHT|wx.LEFT|wx.ALIGN_CENTER_VERTICAL|wx.ALIGN_LEFT, 5 )

self.min_holdText_3 = wx.StaticText( self.findPOC_panel_3, wx.ID_ANY, u".1", wx.DefaultPosition, wx.DefaultSize, wx.ALIGN_LEFT )

self.min_holdText_3.Wrap( -1 )

self.min_holdText_3.SetFont( wx.Font( 12, 74, 90, 90, False, "Calibri" ) )

self.min_holdText_3.SetForegroundColour(wx.SystemSettings.GetColour(wx.SYS_COLOUR_SCROLLBAR ) )

POC_sizer_3.Add( self.min_holdText_3, wx.GBPosition( 7, 0 ), wx.GBSpan( 1,1 ),

wx.ALIGN_LEFT|wx.RIGHT|wx.ALIGN_CENTER_VERTICAL, 5 )

self.hold_spin_3 = FS.FloatSpin( self.findPOC_panel_3, -1 ,value $=1, \min \_v a l=.1$, max_val=600,increment $=.1$, agwStyle=FS.FS_RIGHT )

self.hold_spin_3.SetFormat("\%f")

self.hold_spin_3.SetDigits(2)

self.hold_spin_3.SetFont( wx.Font( 12, 74, 90, 90, False, "Calibri" ) )

self.hold_spin_3.SetForegroundColour( wx.SystemSettings.GetColour(wx.SYS_COLOUR_BTNTEXT) )

self.hold_spin_3.SetBackgroundColour(wx.SystemSettings.GetColour( wx.SYS_COLOUR_BTNSHADOW ) )

POC_sizer_3.Add( self.hold_spin_3, wx.GBPosition( 7,1), wx.GBSpan $(1,1)$,

wx.BOTTOM|wx.ALIGN_CENTER_HORIZONTAL|wx.LEFT|wx.RIGHT, 5 )

self.maxHold_3 = wx.StaticText( self.findPOC_panel_3, wx.ID_ANY, u"600", wx.DefaultPosition, wx.DefaultSize, 0 )

self.maxHold_3.Wrap $(-1)$

self.maxHold_3.SetFont( wx.Font( 12, 74, 90, 90, False, "Calibri" ) )

self.maxHold_3.SetForegroundColour(wx.SystemSettings.GetColour(wx.SYS_COLOUR_SCROLLBAR ) )

POC_sizer_3.Add( self.maxHold_3, wx.GBPosition( 7,2), wx.GBSpan( 1,1 ),

wx.LEFT|wx.TOP|wx.BOTTOM|wx.ALIGN_RIGHT|wx.ALIGN_CENTER_VERTICAL, 5 )

self.radioButton_panel_3 = wx.Panel( self.findPOC_panel_3, wx.ID_ANY, wx.DefaultPosition, wx.DefaultSize, wx.TAB_TRAVERSAL )

self.radioButton_panel_3.SetBackgroundColour(wx.SystemSettings.GetColour(wx.SYS_COLOUR_HIGHLIGHTTEXT ) )

radioButton_sizer_3 $=$ wx.GridSizer $(0,3,0,0)$

self.cycle_3 = wx.RadioButton( self.radioButton_panel_3, wx.ID_ANY, u"Cycle", wx.DefaultPosition, wx.DefaultSize, 0 )

radioButton_sizer_3.Add( self.cycle_3, 0, wx.ALL|wx.ALIGN_CENTER_HORIZONTAL, 5 )

self.cycle_3.SetFont( wx.Font( 12, 74, 90, 90, False, "Calibri" ) )

self.retract_3 = wx.RadioButton( self.radioButton_panel_3, wx.ID_ANY, u"Retract", wx.DefaultPosition, wx.DefaultSize, 0 )

radioButton_sizer_3.Add( self.retract_3, 0, wx.ALL|wx.ALIGN_CENTER_HORIZONTAL, 5 )

self.retract_3.SetFont( wx.Font ( 12, 74, 90, 90, False, "Calibri" ) )

self.extend_3 = wx.RadioButton( self.radioButton_panel_3, wx.ID_ANY, u"Extend", wx.DefaultPosition, wx.DefaultSize, 0 )

radioButton_sizer_3.Add( self.extend_3, 0, wx.ALL|wx.ALIGN_CENTER_HORIZONTAL, 5 )

self.extend_3.SetFont( wx.Font( 12, 74, 90, 90, False, "Calibri" ) )

self.extend_3.SetValue(True)

self.radioButton_panel_3.SetSizer(radioButton_sizer_3 )

self.radioButton_panel_3.Layout() 
radioButton_sizer_3.Fit( self.radioButton_panel_3 )

POC_sizer_3.Add( self.radioButton_panel_3, wx.GBPosition( 8, 0 ), wx.GBSpan( 1,3 ), wx.EXPAND |wx.ALL, 5 )

findPOC_sizer_3.Add( POC_sizer_3, 1, wx.ALIGN_CENTER_HORIZONTAL|wx.ALIGN_CENTER_VERTICAL, 5 )

indentButton_sizer_3 = wx.BoxSizer( wx.VERTICAL )

self.indent_Button_3 = wx.Button( self.findPOC_panel_3, 18, u"Indent", wx.DefaultPosition, wx.Size( 175,60 ), 0 )

self.indent_Button_3.SetFont( wx.Font( 18, 74, 90, 92, False, "Calibri" ) )

self.Bind(wx.EVT_BUTTON,self.displacementIndent3,id=18)

indentButton_sizer_3.Add( self.indent_Button_3, 0, wx.ALIGN_CENTER_HORIZONTAL|wx.ALL, 5 )

findPOC_sizer_3.Add( indentButton_sizer_3, 1, wx.EXPAND, 5 )

self.findPOC_panel_3.SetSizer(findPOC_sizer_3 )

self.findPOC_panel_3.Layout()

step3_sizer.Add( self.findPOC_panel_3, 1, wx.EXPAND, 5 )

self.setPOC_panel_3 = wx.Panel( self.step3_panel, wx.ID_ANY, wx.DefaultPosition, wx.Size( 660,300 ), wx.SIMPLE_BORDER )

setPOC_sizer_3 $=$ wx.BoxSizer( wx.VERTICAL )

setPOC_sizer_3.SetMinSize( wx.Size $(-1,-1)$ )

setPOC_text_sizer_3 = wx.FlexGridSizer $(3,1,0,0)$

setPOC_text_sizer_3.SetFlexibleDirection(wx.BOTH )

setPOC text sizer_3.SetNonFlexibleGrowMode(wx.FLEX_GROWMODE SPECIFIED )

self.setPOC_text_3 = wx.StaticText( self.setPOC_panel_3, wx.ID_ANY, u"Set point of contact and retract 0.6 mm", wx.DefaultPosition,

wx.DefaultSize, wx.ALIGN_LEFT )

self.setPOC_text_3.Wrap( -1)

self.setPOC_text_3.SetFont( wx.Font( 14, 74, 90, 92, False, "Calibri" ) )

self.setPOC_text_3.SetMinSize( wx.Size $(-1,-1)$ )

setPOC_text_sizer_3.Add( self.setPOC_text_3, 1, wx.TOP|wx.BOTTOM|wx.LEFT, 5 )

setPOC_sizer_3.Add( setPOC_text_sizer_3, $1,0,5$ )

setPOC_button_sizer_3 = wx.FlexGridSizer $(3,0,0,0)$

setPOC_button_sizer_3.SetFlexibleDirection(wx.BOTH )

setPOC_button_sizer_3.SetNonFlexibleGrowMode( wx.FLEX_GROWMODE_SPECIFIED )

self.setPOC_button_3 = wx.Button( self.setPOC_panel_3, 19, u"Set", wx.DefaultPosition, wx.Size( 175,60 ), 0 )

self.setPOC_button_3.SetFont( wx.Font (18, 74, 90, 92, False, "Calibri" ) )

self.Bind(wx.EVT_BUTTON,self.setPointOfContactFinal,id=19)

setPOC_button_sizer_3.Add( self.setPOC_button_3, 0, wx.ALIGN_CENTER_HORIZONTAL|wx.TOP|wx.BOTTOM|

wX.ALIGN_CENTER_VERTICAL, 5 )

setPOC_sizer_3.Add( setPOC_button_sizer_3, 0, wx.ALIGN_CENTER_HORIZONTAL|wx.ALIGN_CENTER_VERTICAL, 5 )

self.setPOC_panel_3.SetSizer( setPOC_sizer_3 )

self.setPOC_panel_3.Layout()

step3_sizer.Add( self.setPOC_panel_3, 1, wx.EXPAND, 5 )

self.step3_panel.SetSizer( step3_sizer)

self.step3_panel.Layout()

step3_sizer.Fit( self.step3_panel )

decisionSizer.Add( self.step3_panel, 1, wx.EXPAND, 5 )

self.step4_panel $=$ wx.Panel $($ self.decisionPanel, wx.ID_ANY, wx.DefaultPosition, wx.DefaultSize, wx.TAB_TRAVERSAL )

self.step4_panel.Enable( False )

self.step4_panel.Hide()

decision_sizer $=$ wx.GridSizer $(0,2,0,0)$

decision_sizer.SetMinSize( wx.Size (660,630 ) )

self.forceControl_button_4 = wx.Button( self.step4_panel, 20, u"Force Control", wx.DefaultPosition, wx.Size( 175,60 ), 0 )

self.forceControl_button_4.SetFont( wx.Font( 18, 74, 90, 92, False, "Calibri" ) )

self.Bind(wx.EVT_BUTTON,self.change_forcePage,id=20)

decision_sizer.Add( self.forceControl_button_4, 0, wx.ALL|wx.ALIGN_CENTER_HORIZONTAL|wx.ALIGN_CENTER_VERTICAL, 5 ) self.dispControl_button_4 =wx.Button( self.step4_panel, 21, u"Disp. Control", wx.DefaultPosition, wx.Size (175,60 ), 0 )

self.dispControl_button_4.SetFont( wx.Font( 18, 74, 90, 92, False, "Calibri" ) )

self.Bind(wx.EVT_BUTTON,self.change_dispPage,id=21)

decision_sizer.Add( self.dispControl_button_4, 0, wx.ALL|wx.ALIGN_CENTER_VERTICAL|wx.ALIGN_CENTER_HORIZONTAL, 5 )

self.step4_panel.SetSizer(decision_sizer)

self.step4_panel.Layout()

decision_sizer.Fit( self.step4_panel )

decisionSizer.Add( self.step4_panel, 1, wx.EXPAND|wx.ALL, 5 )

self.step5_force_panel $=$ wx.Panel $($ self.decisionPanel, wx.ID_ANY, wx.DefaultPosition, wx.DefaultSize, wx.TAB_TRAVERSAL )

self.step5_force_panel.Enable( False )

self.step5_force_panel.Hide()

sizer_5 $=$ wx.FlexGridSizer $(5,1,0,0)$

sizer_5.SetFlexibleDirection(wx.BOTH ) 
sizer_5.SetNonFlexibleGrowMode( wx.FLEX_GROWMODE_SPECIFIED )

sizer_5.SetMinSize( wx.Size (660,600))

self.text_panel_5 = wx.Panel( self.step5_force_panel, wx.ID_ANY, wx.DefaultPosition, wx.DefaultSize, wx.TAB_TRAVERSAL )

self.text_panel_5.SetMinSize( wx.Size $(660,-1)$ )

text_sizer_5 $=$ wx.FlexGridSizer $(0,2,0,0)$

text_sizer_5.SetFlexibleDirection(wx.BOTH )

text_sizer_5.SetNonFlexibleGrowMode( wx.FLEX_GROWMODE_SPECIFIED )

text_sizer_5_sub $=$ wx.FlexGridSizer $(0,3,0,0)$

text_sizer_5_sub.SetFlexibleDirection(wx.BOTH )

text_sizer_5_sub.SetNonFlexibleGrowMode( wx.FLEX_GROWMODE_SPECIFIED )

text_sizer_5_sub.SetMinSize( wx.Size $(660,-1)$ )

self.calibrate_text_5 $=$ wx.StaticText( self.text_panel_5, wx.ID_ANY, u"Calibrate", wx.DefaultPosition, wx.Size( $-1,30$ ), 0 )

self.calibrate_text_5.Wrap( -1 )

self.calibrate_text_5.SetFont( wx.Font( 14, 74, 90, 92, False, "Calibri" ) )

text_sizer_5_sub.Add( self.calibrate_text_5, 0, wx.ALL, 5)

Calibrate_level_choiceChoices $=[$ u"Multiple", u"Single" $]$

self.Calibrate_level_choice =wx.Choice( self.text_panel_5, 1001, wx.DefaultPosition,wx.Size $(-1,-1)$, Calibrate_level_choiceChoices, wx.CB_SORT )

self.Calibrate_level_choice.SetSelection( 0 )

self.Calibrate_level_choice.SetFont( wx.Font( 14, 74, 90, 90, False, "Calibri" ) )

self.Bind(wx.EVT_CHOICE,self.changeMult_Single,id=1001)

text_sizer_5_sub.Add( self.Calibrate_level_choice, 0, wx.ALL|wx.ALIGN_CENTER_VERTICAL|wx.ALIGN_CENTER_HORIZONTAL, 5 ) self.indentation_text_5 = wx.StaticText( self.text_panel_5, wx.ID_ANY, u"indentation levels", wx.DefaultPosition, wx.Size ( 150,30 ), 0 ) self.indentation_text_5.Wrap $(-1)$

self.indentation_text_5.SetFont( wx.Font( 14, 74, 90, 92, False, "Calibri" ) )

text_sizer_5_sub.Add( self.indentation_text_5, 0, wx.ALL, 5 )

text_sizer_5.Add( text_sizer_5_sub, 1, wx.EXPAND, 5 )

self.text_panel_5.SetSizer( text_sizer_5 )

self.text_panel_5.Layout()

text_sizer_5.Fit(self.text_panel_5 )

sizer_5.Add( self.text_panel_5, 0,5 )

self.calib_level_text_5 = wx.StaticText( self.step5_force_panel, wx.ID_ANY, u"Calibration values(mN)", wx.DefaultPosition, wx.Size( $-1,-1$ ), 0 )

self.calib_level_text_5.Wrap( -1$)$

self.calib_level_text_5.SetFont( wx.Font( 14, 74, 90, 92, False, "Calibri" ) )

sizer_5.Addd( self.calib_level_text_5, 1, wx.ALIGN_CENTER_VERTICAL|wx.TOP|wx.BOTTOM|wx.RIGHT|wx.LEFT|wx.ALIGN_LEFT, 5 )

self.calibration_param_5_panel =wx.Panel( self.step5_force_panel, wx.ID_ANY, wx.DefaultPosition, wx.DefaultSize, wx.TAB_TRAVERSAL )

self.calibration_param_5_panel.Enable( False )

self.calibration_param_5_panel.Hide()

calibration_param_5_sizer $=$ wx.FlexGridSizer $(4,1,0,0)$

calibration_param_5_sizer.SetFlexibleDirection( wx.BOTH )

calibration_param_5_sizer.SetNonFlexibleGrowMode( wx.FLEX_GROWMODE_SPECIFIED)

params_5_sizer $=$ wx.GridBagSizer $(0,0)$

params_5_sizer.SetFlexibleDirection( wx.BOTH )

params_5_sizer.SetNonFlexibleGrowMode(wx.FLEX_GROWMODE_SPECIFIED )

self.highest_Force_5 = wx.StaticText( self.calibration_param_5_panel, wx.ID_ANY, u"highest force", wx.DefaultPosition, wx.DefaultSize, 0 ) self.highest_Force_5.Wrap( -1 )

self.highest_Force_5.SetFont( wx.Font( 12, 74, 90, 92, False, "Calibri" ) )

params_5_sizer.Add( self.highest_Force_5, wx.GBPosition( 0,1 ), wx.GBSpan $(1,1)$, wx.TOP|wx.RIGHT|wx.LEFT|wx.ALIGN_CENTER_HORIZONTAL, 5 )

self.highestForce_textCtrl_5 = wx.TextCtrl( self.calibration_param_5_panel, wx.ID_ANY, u"50", wx.DefaultPosition, wx.DefaultSize, 0 )

self.highestForce_textCtrl_5.SetFont( wx.Font( 14, 74, 90, 90, False, "Calibri" ) )

params_5_sizer.Add( self.highestForce_textCtrl_5, wx.GBPosition( 1,1 ), wx.GBSpan ( 1,1),

wx.ALIGN_CENTER_HORIZONTAL|wx.RIGHT|wx.LEFT, 5 )

self.min_text_5 = wx.StaticText( self.calibration_param_5_panel, wx.ID_ANY, u"Min", wx.DefaultPosition, wx.DefaultSize, wx.ALIGN_LEFT )

self.min_text_5.Wrap (-1)

self.min_text_5.SetFont( wx.Font( 12, 74, 90, 90, False, "Calibri" ) )

self.min_text_5.SetForegroundColour( wx.SystemSettings.GetColour( wx.SYS_COLOUR_SCROLLBAR ) )

self.min_text_5.SetMinSize(wx.Size( 100,-1 ))

params_5_sizer.Add( self.min_text_5, wx.GBPosition( 2, 0 ), wx.GBSpan( 1, 1),wx.ALL|wx.ALIGN_LEFT, 5 )

self.Velocity_text_5 = wx.StaticText( self.calibration_param_5_panel, wx.ID_ANY, u"velocity $(\mathrm{mm} / \mathrm{s})$ ", wx.DefaultPosition, wx.DefaultSize, 0 ) self.Velocity_text_5.Wrap( -1 )

self.Velocity_text_5.SetFont( wx.Font( 12, 74, 90, 92, False, "Calibri" ) )

params_5_sizer.Add( self.Velocity_text_5, wx.GBPosition( 2, 1 ), wx.GBSpan( 1,1 ),

wx.ALIGN_CENTER_HORIZONTAL|wx.TOP|wx.RIGHT|wx.LEFT, 5 ) 
self.max_text_5 =wx.StaticText( self.calibration_param_5_panel, wx.ID_ANY, u"Max", wx.DefaultPosition, wx.DefaultSize,

wx.ALIGN_RIGHT )

self.max_text_5.Wrap $(-1)$

self.max_text_5.SetFont( wx.Font( 12, 74, 90, 90, False, "Calibri" ) )

self.max_text_5.SetForegroundColour(wx.SystemSettings.GetColour( wx.SYS_COLOUR_SCROLLBAR ))

self.max_text_5.SetMinSize(wx.Size( $100,-1)$ )

params_5_sizer.Add( self.max_text_5,wx.GBPosition( 2, 2), wx.GBSpan( 1, 1 ), wx.ALL|wx.ALIGN_RIGHT, 5 )

self.min_val_1_5 = wx.StaticText( self.calibration_param_5_panel, wx.ID_ANY, u"1", wx.DefaultPosition, wx.DefaultSize, 0 )

self.min_val_1_5.Wrap( -1 )

self.min_val_1_5.SetFont( wx.Font( 12, 74, 90, 90, False, "Calibri" ) )

self.min_val_1_5.SetForegroundColour((64,64,64))

params_5_sizer.Add( self.min_val_1_5, wx.GBPosition( 3, 0 ), wx.GBSpan( 1, 1 ), wx.ALL|wx.ALIGN_LEFT, 5 )

self.vel_choice_5Choices $=$ [ u"0.5",u"0.75",u"1", u"2", u"3", u"4", u"5", wx.EmptyString ]

self.vel_choice_5 = wx.Choice( self.calibration_param_5_panel, wx.ID_ANY, wx.DefaultPosition, wx.Size( 100,-1 ), self.vel_choice_5Choices, 0 )

self.vel_choice_5.SetSelection( 0 )

self.vel_choice_5.SetFont( wx.Font( 14, 74, 90, 90, False, "Calibri" ) )

params_5_sizer.Add( self.vel_choice_5, wx.GBPosition( 3,1),wx.GBSpan( 1,1 ),

wx.ALIGN_CENTER_HORIZONTAL/wx.BOTTOM|wx.RIGHT|wx.LEFT, 5 )

self.max_val_1_5 = wx.StaticText( self.calibration_param_5_panel, wx.ID_ANY, u"5", wx.DefaultPosition, wx.DefaultSize, 0 )

self.max_val_1_5.Wrap( -1 )

self.max_val_1_5.SetFont( wx.Font( 12, 74, 90, 90, False, "Calibri" ) )

self.max_val_1_5.SetForegroundColour(wx.SystemSettings.GetColour(wx.SYS_COLOUR_SCROLLBAR ) )

params_5_sizer.Add( self.max_val_1_5, wx.GBPosition( 3, 2 ), wx.GBSpan( 1, 1 ), wx.ALL|wx.ALIGN_RIGHT, 5)

calibration_param_5_sizer.Add( params_5_sizer, 0, wx.ALIGN_CENTER_VERTICAL|wx.ALIGN_CENTER_HORIZONTAL, 5 )

calibrate_button_sizer_1 $=$ wx.BoxSizer(wx.VERTICAL )

self.calibrate_button_5 $=$ wx.Button( self.calibration_param_5_panel, 23, u"Calibrate", wx.DefaultPosition, wx.Size (175,60 ), 0 )

self.calibrate_button_5.SetFont( wx.Font( 18, 74, 90, 92, False, "Calibri" ) )

self.Bind(wx.EVT_BUTTON,self.Calibrate_Single,id=23)

calibrate_button_sizer_1.Add( self.calibrate_button_5, 0,wx.ALL|wx.ALIGN_CENTER_HORIZONTAL, 5)

calibration_param_5_sizer.Add( calibrate_button_sizer_1, 0, wx.ALIGN_CENTER_HORIZONTAL, 5 )

self.calibration_param_5_panel.SetSizer( calibration_param_5_sizer )

self.calibration_param_5_panel.Layout()

calibration_param_5_sizer.Fit( self.calibration_param_5_panel )

sizer_5.Add( self.calibration_param_5_panel, 1, wx.ALL/wx.ALIGN_CENTER_HORIZONTAL, 5 )

self.calib_param_mutliple_5 $=$ wx.Panel( self.step5_force_panel, wx.ID_ANY, wx.DefaultPosition, wx.DefaultSize, wx.TAB_TRAVERSAL )

calib_sizer_multiple_5 = wx.FlexGridSizer $(5,1,0,0)$

calib_sizer_multiple_5.SetFlexibleDirection(wx.BOTH )

calib_sizer_multiple_5.SetNonFlexibleGrowMode( wx.FLEX_GROWMODE_SPECIFIED )

partition_5_mult_sizer $=$ wx.FlexGridSizer $(0,2,0,0)$

partition_5_mult_sizer.SetFlexibleDirection( wx.BOTH )

partition_5_mult_sizer.SetNonFlexibleGrowMode(wx.FLEX_GROWMODE_SPECIFIED)

self.how_to_part_text_5_mult $=$ wx.StaticText( self.calib_param_mutliple_5, wx.ID_ANY, u"How to partition forces?", wx.DefaultPosition, wx.DefaultSize, 0 )

self.how_to_part_text_5_mult.Wrap( -1 )

self.how_to_part_text_5_mult.SetFont( wx.Font( 14, 74, 90, 92, False, "Calibri" ) )

partition_5_mult_sizer.Add( self.how_to_part_text_5_mult, 0, wx.ALL, 5)

line_expo_choiceChoices $=[$ u"Linearly", u"Exponentially" ]

self.line_expo_choice $=$ wx.Choice $($ self.calib_param_mutliple_5, wx.ID_ANY, wx.DefaultPosition, wx.DefaultSize, line_expo_choiceChoices, 0 )

self.line_expo_choice.SetSelection( 0 )

self.line_expo_choice.SetFont( wx.Font (14, 74, 90, 90, False, "Calibri" ) )

partition_5_mult_sizer.Add( self.line_expo_choice, 0, wx.ALIGN_RIGHT|wx.ALIGN_CENTER_VERTICAL|wx.ALL, 5 )

calib_sizer_multiple_5.Add ( partition_5_mult_sizer, 1, wx.EXPAND, 5 )

params_5_sizer_mult $=$ wx.GridBagSizer $(0,0)$

params_5_sizer_mult.SetFlexibleDirection(wx.BOTH )

params_5_sizer_mult.SetNonFlexibleGrowMode( wx.FLEX_GROWMODE_SPECIFIED)

self.contact_Force_5_mult $=$ wx.StaticText( self.calib_param_mutliple_5, wx.ID_ANY, u"contact force", wx.DefaultPosition, wx.DefaultSize, 0 )

self.contact_Force_5_mult.Wrap( -1)

self.contact_Force_5_mult.SetFont( wx.Font( 12, 74, 90, 92, False, "Calibri" ) )

params_5_sizer_mult.Add( self.contact_Force_5_mult, wx.GBPosition( 0,0 ), wx.GBSpan $(1,1)$,

wx.TOP|wx.RIGHT|wx.LEFT|wx.ALIGN_CENTER_HORIZONTAL, 5 )

self.highest_Force_5_mult $=$ wx.StaticText( self.calib_param_mutliple_5, wx.ID_ANY, u"highest force", wx.DefaultPosition, wx.DefaultSize, 0 )

self.highest_Force_5_mult.Wrap( -1 ) 
self.highest_Force_5_mult.SetFont(wx.Font( 12, 74, 90, 92, False, "Calibri" ) )

params_5_sizer_mult.Add( self.highest_Force_5_mult, wx.GBPosition( 0,2 ), wx.GBSpan( 1,1 ),

wx.TOP|wx.RIGHT|wx.LEFT|wx.ALIGN_CENTER_HORIZONTAL, 5 )

self.low_force_text_mult $=$ wx.StaticText( self.calib_param_mutliple_5, wx.ID_ANY, u"lowest force", wx.DefaultPosition, wx.DefaultSize, 0 )

self.low_force_text_mult.Wrap( -1$)$

self.low_force_text_mult.SetFont( wx.Font( 12, 74, 90, 92, False, "Calibri" ) )

params_5_sizer_mult.Add( self.low_force_text_mult, wx.GBPosition( 0,1$)$, wx.GBSpan( 1,1$)$,

wx.ALL|wx.ALIGN_CENTER_HORIZONTAL, 5 )

self.force_interval_text_mult $=$ wx.StaticText( self.calib_param_mutliple_5, wx.ID_ANY, u"force interval", wx.DefaultPosition, wx.DefaultSize, 0 )

self.force_interval_text_mult.Wrap( -1 )

self.force_interval_text_mult.SetFont( wx.Font( 12, 74, 90, 92, False, "Calibri" ) )

params_5_sizer_mult.Add( self.force_interval_text_mult, wx.GBPosition $(0,3)$, wx.GBSpan( 1,1 ),

wx.ALL/wx.ALIGN CENTER HORIZONTAL, 5 )

self.POCForce_textCtrl_5_mult $=$ wx.TextCtrl( self.calib_param_mutliple_5, wx.ID_ANY, u"50", wx.DefaultPosition, wx.DefaultSize,

wx.ALIGN_RIGHT|wx.TE_READONLY)

self.POCForce_textCtrl_5_mult.SetFont( wx.Font( 14, 74, 90, 90, False, "Calibri" ) )

params_5_sizer_mult.Add( self.POCForce_textCtrl_5_mult, wx.GBPosition( 1,0 ), wx.GBSpan( 1, 1 ), wx.RIGHT|wx.LEFT, 5 )

self.lowestForce_textCtrl_5_mult $=$ wx.TextCtrl( self.calib_param_mutliple_5, wx.ID_ANY, u"50", wx.DefaultPosition, wx.DefaultSize,

wx.ALIGN_RIGHT )

self.lowestForce_textCtrl_5_mult.SetFont(wx.Font( 14, 74, 90, 90, False, "Calibri" ) )

params_5_sizer_mult.Add( self.lowestForce_textCtrl_5_mult, wx.GBPosition( 1, 1), wx.GBSpan( 1, 1), wx.RIGHT|wx.LEFT, 5 )

self.highestForce_textCtrl_5_mult = wx.TextCtrl( self.calib_param_mutliple_5, wx.ID_ANY, u"50", wx.DefaultPosition, wx.DefaultSize,

wx.ALIGN_RIGHT)

self.highestForce_textCtrl_5_mult.SetFont(wx.Font( 14, 74, 90, 90, False, "Calibri" ) )

params_5_sizer_mult.Add( self.highestForce_textCtrl_5_mult, wx.GBPosition( 1,2),wx.GBSpan( 1, 1 ),

wx.ALIGN_CENTER_HORIZONTAL|wx.RIGHT|wx.LEFT, 5 )

self.interval_textCtrl_5_mult =wx.TextCtrl( self.calib_param_mutliple_5, wx.ID_ANY, u"50", wx.DefaultPosition, wx.DefaultSize,

wx.ALIGN_RIGHT )

self.interval_textCtrl_5_mult.SetFont(wx.Font( 14, 74, 90, 90, False, "Calibri" ) )

params_5_sizer_mult.Add( self.interval_textCtrl_5_mult, wx.GBPosition( 1, 3 ), wx.GBSpan( 1, 1 ), wx.RIGHT|wx.LEFT, 5 )

calib_sizer_multiple_5.Add( params_5_sizer_mult, 1, wx.EXPAND, 5 )

params_5_sizer_mult_1 $=$ wx.GridBagSizer $(0,0)$

params_5_sizer_mult_1.SetFlexibleDirection(wx.BOTH )

params_5_sizer_mult_1.SetNonFlexibleGrowMode( wx.FLEX_GROWMODE_SPECIFIED )

self.min_text_5_mult $=$ wx.StaticText $($ self.calib_param_mutliple_5, wx.ID_ANY, u"Min", wx.DefaultPosition, wx.DefaultSize, 0 )

self.min_text_5_mult.Wrap( -1 )

self.min_text_5_mult.SetFont( wx.Font( 12, 74, 90, 90, False, "Calibri" ) )

self.min_text_5_mult.SetForegroundColour $(96,96,96))$

self.min_text_5_mult.SetMinSize( wx.Size $(100,-1)$ )

params_5_sizer_mult_1.Add( self.min_text_5_mult, wx.GBPosition $(0,0)$, wx.GBSpan $(1,1)$, wx.ALL|wx.ALIGN_LEFT, 5 )

self.Velocity_text_5_mult $=$ wx.StaticText( self.calib_param_mutliple_5, wx.ID_ANY, u"velocity $(\mathrm{mm} / \mathrm{s}) "$, wx.DefaultPosition, wx.DefaultSize, 0 )

self.Velocity_text_5_mult.Wrap( -1 )

self.Velocity_text_5_mult.SetFont( wx.Font( 12, 74, 90, 92, False, "Calibri" ) )

params_5_sizer_mult_1.Add( self.Velocity_text_5_mult, wx.GBPosition $(0,1)$, wx.GBSpan $(1,1)$,

wx.ALIGN_CENTER_HORIZONTAL|wx.TOP|wx.RIGHT|wx.LEFT, 5 )

self.max_text_5_mult = wx.StaticText( self.calib_param_mutliple_5, wx.ID_ANY, u"Max", wx.DefaultPosition, wx.DefaultSize, wx.ALIGN_RIGHT )

self.max_text_5_mult.Wrap( -1$)$

self.max_text_5_mult.SetFont( wx.Font (12, 74, 90, 90, False, "Calibri" ) )

self.max_text_5_mult.SetForegroundColour $(96,96,96))$

self.max_text_5_mult.SetMinSize( wx.Size $(100,-1)$ )

params_5_sizer_mult_1.Add( self.max_text_5_mult, wx.GBPosition( 0, 2 ), wx.GBSpan( 1,1 ), wx.ALL|wx.ALIGN_RIGHT, 5 )

self.min_val_1_5_mult $=$ wx.StaticText $($ self.calib_param_mutliple_5, wx.ID_ANY, u"1", wx.DefaultPosition, wx.DefaultSize, 0 )

self.min_val_1_5_mult.Wrap $(-1)$

self.min_val_1_5_mult.SetFont( wx.Font( 12, 74, 90, 90, False, "Calibri" ) )

self.min_val_1_5_mult.SetForegroundColour $((96,96,96))$

params_5_sizer_mult_1.Add( self.min_val_1_5_mult, wx.GBPosition( 1,0 ), wx.GBSpan( 1,1 ), wx.ALL|wx.ALIGN_LEFT, 5 )

self.vel_choice_5_multChoices = [ u"0.5",u"0.75",u"1", u"2", u"3", u"4", wx.EmptyString ]

self.vel_choice_5_mult $=$ wx.Choice( self.calib_param_mutliple_5, wx.ID_ANY, wx.DefaultPosition, wx.Size( 100,-1),

self.vel_choice_5_multChoices, 0 )

self.vel_choice_5_mult.SetSelection( 0 )

self.vel_choice_5_mult.SetFont( wx.Font( 14, 74, 90, 90, False, "Calibri" ) )

params_5_sizer_mult_1.Add( self.vel_choice_5_mult, wx.GBPosition( 1, 1), wx.GBSpan( 1, 1),

wx.ALIGN_CENTER_HORIZONTAL $|w x . B O T T O M| w x . R I G H T \mid w x . L E F T, ~ 5)$

self.max_val_1_5_mult $=$ wx.StaticText( self.calib_param_mutliple_5, wx.ID_ANY, u"5", wx.DefaultPosition, wx.DefaultSize, 0 )

self.max_val_1_5_mult.Wrap(-1) 
self.max_val_1_5_mult.SetFont( wx.Font( 12, 74, 90, 90, False, "Calibri" ) )

self.max_val_1_5_mult.SetForegroundColour $((96,96,96))$

params_5_sizer_mult_1.Add( self.max_val_1_5_mult, wx.GBPosition( 1,2), wx.GBSpan ( 1, 1 ), wx.ALL|wx.ALIGN_RIGHT, 5 )

calib_sizer_multiple_5.Add( params_5_sizer_mult_1, 1, wx.ALIGN_CENTER_HORIZONTAL, 5 )

calibrate_button_sizer_mult $=$ wx.BoxSizer $($ wx.VERTICAL $)$

self.calibrate_button_5_mult $=$ wx.Button $($ self.calib_param_mutliple_5, 24, u"Calibrate", wx.DefaultPosition, wx.Size $(175,60), 0$ )

self.calibrate_button_5_mult.SetFont( wx.Font( 18, 74, 90, 92, False, "Calibri" ) )

self.Bind(wx.EVT_BUTTON,self.Calibrate_Mult,id=24)

calibrate_button_sizer_mult.Add( self.calibrate_button_5_mult, 0, wx.ALL|wx.ALIGN_CENTER_HORIZONTAL, 5 )

calib_sizer_multiple_5.Add( calibrate_button_sizer_mult, 1, wx.EXPAND, 5 )

self.calib_param_mutliple_5.SetSizer(calib_sizer_multiple_5 )

self.calib_param_mutliple_5.Layout()

calib_sizer_multiple_5.Fit( self.calib_param_mutliple_5)

sizer_5.Add( self.calib_param_mutliple_5, 0 , wx.ALL|wx.ALIGN_CENTER_HORIZONTAL, 5 )

self.m_panel79 = wx.Panel( self.step5_force_panel, wx.ID_ANY, wx.DefaultPosition, wx.DefaultSize,

wx.TAB_TRAVERSAL|wx.SIMPLE_BORDER )

bSizer55 $=$ wx.BoxSizer $(w x$. VERTICAL $)$

self.levels_calibrate_text_6 $=$ wx.StaticText( self.m_pane179, wx.ID_ANY, u"Force levels calibrated", wx.DefaultPosition, wx.DefaultSize, wx.ALIGN_LEFT )

self.levels_calibrate_text_6.Wrap( -1 )

self.levels_calibrate_text_6.SetFont( wx.Font( 14, 74, 90, 92, False, "Calibri" ) )

bSizer55.Add( self.levels_calibrate_text_6, 0, wx.RIGHT|wx.LEFT, 5 )

self.calib_table_panel_5 = wx.ScrolledWindow( self.m_panel79, wx.ID_ANY, wx.DefaultPosition, $(370,300)$,

wx.HSCROLL|wx.SIMPLE_BORDER/wx.VSCROLL )

self.calib_table_panel_5.SetScrollRate $(5,5)$

calib_table_sizer_5 = wx.FlexGridSizer $(0,7,0,0)$

calib_table_sizer_5.SetFlexibleDirection(wx.BOTH )

calib_table_sizer_5.SetNonFlexibleGrowMode( wx.FLEX_GROWMODE_SPECIFIED)

self.calib_table_panel_5.SetMinSize(wx.Size( 370,300 ) )

self.calib_ID_panel_5 = wx.Panel( self.calib_table_panel_5, wx.ID_ANY, wx.DefaultPosition, wx.DefaultSize, wx.TAB_TRAVERSAL )

self.calib_ID_panel_5.SetBackgroundColour(wx.SystemSettings.GetColour(wx.SYS_COLOUR_SCROLLB AR ) )

self.calib_ID_panel_5.SetMinSize( wx.Size $(-1,600))$

self.calib_ID_sizer_5 $=$ wx.BoxSizer( wx.VERTICAL )

self.calib_ID_sizer_5.SetMinSize( wx.Size $(-1,600)$ )

self.calib_ID_header_5 = wx.StaticText( self.calib_ID_panel_5, wx.ID_ANY, u"ID", wx.DefaultPosition, wx.Size( 50,30 ),

wx.ALIGN_CENTRE)

self.calib_ID_header_5.Wrap( -1 )

self.calib_ID_header_5.SetBackgroundColour(wx.SystemSettings.GetColour(wx.SYS_COLOUR_SCROLLBAR ) )

self.calib_ID_sizer_5.Add( self.calib_ID_header_5, 0, wx.ALL, 5 )

self.calib_ID_staticline_5 = wx.StaticLine( self.calib_ID_panel_5, wx.ID_ANY, wx.DefaultPosition, wx.DefaultSize, wx.LI_HORIZONTAL ) self.calib_ID_sizer_5.Add( self.calib_ID_staticline_5, 0, wx.EXPAND|wx.TOP|wx.BOTTOM, 5 )

self.calib_ID_panel_5.SetSizer( self.calib_ID_sizer_5 )

self.calib_ID_panel_5.Layout()

self.calib_ID_sizer_5.Fit( self.calib_ID_panel_5 )

calib_table_sizer_5.Add( self.calib_ID_panel_5, 1, wx.EXPAND|wx.BOTTOM, 5 )

self.calib_force_panel_5 = wx.Panel( self.calib_table_panel_5, wx.ID_ANY, wx.DefaultPosition, wx.DefaultSize, wx.TAB_TRAVERSAL )

self.calib_force_panel_5.SetBackgroundColour(wx.SystemSettings.GetColour(wx.SYS_COLOUR_3DLIGHT ) )

self.calib_force_panel_5.SetMinSize(wx.Size( 100,600))

self.calib_force_sizer_5 = wx.BoxSizer( wx.VERTICAL )

self.calib_force_sizer_5.SetMinSize( wx.Size (100,600))

self.calib_Force_header_5 = wx.StaticText( self.calib_force_panel_5, wx.ID_ANY, u"forceln(mN)", wx.DefaultPosition, wx.Size( $-1,30$ ),

wX.ALIGN_CENTRE)

self.calib_Force_header_5.Wrap $(-1)$

self.calib_force_sizer_5.Add( self.calib_Force_header_5, 0, wx.ALL|wx.ALIGN_CENTER_HORIZONTAL, 5)

self.calib_force_staticline $=$ wx.StaticLine $($ self.calib_force_panel_5, wx.ID_ANY, wx.DefaultPosition, wx.DefaultSize, wx.LI_HORIZONTAL )

self.calib_force_sizer_5.Add( self.calib_force_staticline, 0, wx.EXPAND|wx.TOP|wx.BOTTOM, 5 )

self.calib_force_panel_5.SetSizer( self.calib_force_sizer_5 )

self.calib_force_panel_5.Layout()

self.calib_force_sizer_5.Fit( self.calib_force_panel_5 )

calib_table_sizer_5.Add( self.calib_force_panel_5, 1, wx.EXPAND|wx.BOTTOM, 5 )

self.calib_disp_panel_5 =wx.Panel( self.calib_table_panel_5, wx.ID_ANY, wx.DefaultPosition, wx.DefaultSize, wx.TAB_TRAVERSAL )

self.calib_disp_panel_5.SetBackgroundColour(wx.SystemSettings.GetColour( wx.SYS_COLOUR_SCROLLBAR ) )

self.calib_disp_panel_5.SetMinSize (wx.Size $(-1,600))$

self.calib_disp_sizer_5 = wx.BoxSizer(wx.VERTICAL )

self.calib_disp_sizer_5.SetMinSize( wx.Size (100,600) )

self.calib_disp_header_5 = wx.StaticText( self.calib_disp_panel_5, wx.ID_ANY, u"displacement $\ln (\mathrm{mm})$ ", wx.DefaultPosition, wx.Size( $-1,30$ ), wX.ALIGN_CENTRE ) 
self.calib_disp_header_5.Wrap( -1 )

self.calib_disp_sizer_5.Add( self.calib_disp_header_5, 0, wx.ALL|wx.ALIGN_CENTER_HORIZONTAL, 5 )

self.calib_disp_staticline_5 = wx.StaticLine( self.calib_disp_panel_5, wx.ID_ANY, wx.DefaultPosition, wx.DefaultSize, wx.LI_HORIZONTAL )

self.calib_disp_sizer_5.Add( self.calib_disp_staticline_5, 0, wx.EXPAND|wx.TOP|wx.BOTTOM, 5 )

self.calib_disp_panel_5.SetSizer( self.calib_disp_sizer_5 )

self.calib_disp_panel_5.Layout()

self.calib_disp_sizer_5.Fit( self.calib_disp_panel_5 )

calib_table_sizer_5.Add( self.calib_disp_panel_5, 1, wx.EXPAND|wx.BOTTOM, 5 )

self.calib_vel_panel_5 $=$ wx.Panel( self.calib_table_panel_5, wx.ID_ANY, wx.DefaultPosition, wx.DefaultSize, wx.TAB_TRAVERSAL )

self.calib_vel_panel_5.SetMinSize (wx.Size $(-1,600))$

self.calib_vel_sizer_5 = wx.BoxSizer( wx.VERTICAL )

self.calib_vel_sizer_5.SetMinSize( wx.Size (100,600))

self.calib_vel_header_5 = wx.StaticText( self.calib_vel_panel_5, wx.ID_ANY, u"velocity $\backslash n(\mathrm{~mm} / \mathrm{s}) "$, wx.DefaultPosition, wx.Size( 50,30 ),

wx.ALIGN_CENTRE )

self.calib_vel_header_5.Wrap( -1 )

self.calib_vel_sizer_5.Add( self.calib_vel_header_5, 0, wx.ALL|wx.ALIGN_CENTER_HORIZONTAL, 5)

self.calib_vel_staticline_5 = wx.StaticLine( self.calib_vel_panel_5, wx.ID_ANY, wx.DefaultPosition, wx.DefaultSize, wx.LI_HORIZONTAL )

self.calib_vel_sizer_5.Add( self.calib_vel_staticline_5, 0, wx.EXPAND|wx.TOP|wx.BOTTOM, 5 )

self.calib_vel_panel_5.SetSizer( self.calib_vel_sizer_5 )

self.calib_vel_panel_5.Layout()

self.calib_vel_sizer_5.Fit( self.calib_vel_panel_5 )

calib_table_sizer_5.Add( self.calib_vel_panel_5, 1, wx.EXPAND|wx.BOTTOM, 5 )

self.calib_table_panel_5.SetSizer( calib_table_sizer_5 )

self.calib_table_panel_5.Layout()

calib_table_sizer_5.Fit( self.calib_table_panel_5 )

bSizer55.Add( self.calib_table_panel_5, 0, wx.ALIGN_CENTER_VERTICAL|wx.ALIGN_CENTER_HORIZONTAL, 5 )

self.m_panel79.SetSizer( bSizer55 )

self.m_panel79.Layout()

bSizer55.Fit( self.m_panel79)

sizer_5.Add( self.m_panel79, 1, wx.EXPAND, 5 )

self.step5_force_panel.SetSizer( sizer_5 )

self.step5_force_panel.Layout()

sizer_5.Fit( self.step5_force_panel)

decisionSizer.Add( self.step5_force_panel, 1, wx.EXPAND, 5 )

self.step6_force_panel $=$ wx.Notebook( self.decisionPanel, wx.ID_ANY, wx.DefaultPosition, wx.DefaultSize, wx.NB_TOP )

self.step6_force_panel.Enable( False )

self.step6_force_panel.Hide()

self.Force_Displacement $=$ wx.Panel( self.step6_force_panel, wx.ID_ANY, wx.DefaultPosition, wx.DefaultSize, wx.TAB_TRAVERSAL )

Force_Disp_tab_sizer_6 $=$ wx.GridBagSizer $(0,0)$

Force_Disp_tab_sizer_6.SetFlexibleDirection(wx.BOTH )

Force_Disp_tab_sizer_6.SetNonFlexibleGrowMode( wx.FLEX_GROWMODE_SPECIFIED )

text_sizer_5_sub1 $=$ wx.FlexGridSizer $(0,3,0,0)$

text_sizer_5_sub1.SetFlexibleDirection( wx.BOTH )

text_sizer_5_sub1.SetNonFlexibleGrowMode( wx.FLEX_GROWMODE_SPECIFIED )

text_sizer_5_sub1.SetMinSize(wx.Size $(660,-1)$ )

self.calibrate_text_51 $=$ wx.StaticText( self.Force_Displacement, wx.ID_ANY, u"Choose", wx.DefaultPosition, wx.Size $(-1,30$ ), 0 )

self.calibrate_text_51.Wrap( -1 )

self.calibrate_text_51.SetFont( wx.Font( 14, 74, 90, 92, False, "Calibri" ) )

text_sizer_5_sub1.Add( self.calibrate_text_51,0,wx.ALL, 5 )

type_mec_IndentChoices $=[$ u"force", u"displacement" ]

self.type_mec_Indent $=$ wx.Choice( self.Force_Displacement, wx.ID_ANY, wx.DefaultPosition, wx.Size $(-1,-1)$, type_mec_IndentChoices, wx.CB_SORT)

self.type_mec_Indent.SetSelection( 0 )

self.type_mec_Indent.SetFont(wx.Font( 14, 74, 90, 90, False, "Calibri" ) )

text_sizer_5_sub1.Add( self.type_mec_Indent, 0, wx.ALL|wx.ALIGN_CENTER_VERTICAL|wx.ALIGN_CENTER_HORIZONTAL, 5 )

self.indentation_text_51 = wx.StaticText( self.Force_Displacement, wx.ID_ANY, u"mechanical stimulation", wx.DefaultPosition, wx.Size( $200,30), 0$ )

self.indentation_text_51.Wrap( -1$)$

self.indentation_text_51.SetFont( wx.Font( 14, 74, 90, 92, False, "Calibri" ) )

text_sizer_5_sub1.Add( self.indentation_text_51,0,wx.ALL, 5 )

Force_Disp_tab_sizer_6.Add( text_sizer_5_sub1, wx.GBPosition( 0, 0 ), wx.GBSpan( 1,1 ), wx.EXPAND, 5 )

force_disp_indentation_parm_6 = wx.GridBagSizer $(0,0)$

force_disp_indentation_parm_6.SetFlexibleDirection(wx.BOTH )

force_disp_indentation_parm_6.SetNonFlexibleGrowMode( wx.FLEX_GROWMODE_SPECIFIED)

self.max_text12 = wx.StaticText( self.Force_Displacement, wx.ID_ANY, u"Max", wx.DefaultPosition, wx.DefaultSize, wx.ALIGN_RIGHT)

self.max_text12.Wrap( -1$)$

self.max_text12.SetFont( wx.Font( 12, 74, 90, 90, False, "Calibri" ) )

self.max_text12.SetForegroundColour $(96,96,96))$ 
force_disp_indentation_parm_6.Add( self.max_text12, wx.GBPosition( 4, 2), wx.GBSpan( 1,1 ), wx.ALL|wx.ALIGN_CENTER_VERTICAL|wx.ALIGN_RIGHT, 5 ) self.displacement_text12 = wx.StaticText( self.Force_Displacement, wx.ID_ANY, u"force (mN)", wx.DefaultPosition, wx.DefaultSize, wx.ALIGN_LEFT )

self.displacement_text12.Wrap (-1)

self.displacement_text12.SetFont( wx.Font( 12, 74, 90, 90, False, "Calibri" ) )

force_disp_indentation_parm_6.Add( self.displacement_text12, wx.GBPosition $(2,0)$, wx.GBSpan $(1,1)$,

wx.LEFT|wx.RIGHT|wx.ALIGN_LEFT|wx.ALIGN_BOTTOM, 5 )

self.min_text12 = wx.StaticText( self.Force_Displacement, wx.ID_ANY, u"Min", wx.DefaultPosition, wx.DefaultSize, wx.ALIGN_LEFT )

self.min_text12.Wrap ( -1$)$

self.min_text12.SetFont( wx.Font( 12, 74, 90, 90, False, "Calibri" ) )

self.min_text12.SetForegroundColour( $(96,96,96))$

force_disp_indentation_parm_6.Add( self.min_text12, wx.GBPosition $(4,0)$, wx.GBSpan $(1,1)$,

wx.ALL|wx.ALIGN_LEFT|wx.ALIGN_CENTER_VERTICAL, 5 )

\#self.minDisp_text12 =wx.StaticText( self.Force_Displacement, wx.ID_ANY, u"0.01", wx.DefaultPosition, wx.Size( $-1,-1$ ), wx.ALIGN_LEFT )

\#self.minDisp_text12.Wrap( -1 )

\#self.minDisp_text12.SetFont( wx.Font( 12, 74, 90, 90, False, "Calibri" ) )

\#self.minDisp_text12.SetForegroundColour(wx.SystemSettings.GetColour( wx.SYS_COLOUR_SCROLLBAR ) )

\#self.minDisp_text12.SetMinSize( wx.Size ( 100,-1 ))

\#force_disp_indentation_parm_6.Add( self.minDisp_text12, wx.GBPosition( 3,0 ), wx.GBSpan( 1,1 ),

wx.ALIGN_CENTER_HORIZONTAL|wx.TOP|wx.BOTTOM|wx.ALIGN_CENTER_VERTICAL, 5 )

\#self.minVel_text12 = wx.StaticText( self.Force_Displacement, wx.ID_ANY, u"1.0", wx.DefaultPosition, wx.Size ( $-1,-1)$,

wx.ALIGN_CENTRE )

\#self.minVel_text12.Wrap ( -1)

\#self.minVel_text12.SetFont(wx.Font( 12, 74, 90, 90, False, "Calibri" ) )

\#self.minVel_text12.SetForegroundColour(wx.SystemSettings.GetColour( wx.SYS_COLOUR_SCROLLBAR ) )

\#force_disp_indentation_parm_6.Add( self.minVel_text12, wx.GBPosition( 5, 0 ), wx.GBSpan( 1,1 ),

wx.ALIGN_CENTER_HORIZONTAL/wx.TOP|wx.BOTTOM|wx.LEFT|wx.ALIGN_CENTER_VERTICAL, 5 )

self.F_D_force_textCtrl_6 $=$ wx.TextCtrl( self.Force_Displacement, wx.ID_ANY, wx.EmptyString, wx.DefaultPosition, wx.Size( $90,-1$ ), wx.TE_RIGHT|wx.TE_READONLY)

self.F_D_force_textCtrl_6.SetFont( wx.Font( 14, 74, 90, 90, False, "Calibri" ) )

self.F_D_force_textCtrl_6.SetBackgroundColour( wx.SystemSettings.GetColour( wx.SYS_COLOUR_SCROLLBAR ) )

force_disp_indentation_parm_6.Add( self.F_D_force_textCtrl_6, wx.GBPosition $(3,0)$, wx.GBSpan $(1,1)$,

wx.BOTTOM|wx.LEFT|wx.RIGHT|wx.ALIGN_CENTER_HORIZONTAL, 5 )

self.F_D_disp_textCtrl_6 = wx.TextCtrl( self.Force_Displacement, wx.ID_ANY, wx.EmptyString, wx.DefaultPosition, wx.Size( $90,-1$ ), wx.TE_RIGHT|wx.TE_READONLY)

self.F_D_disp_textCtrl_6.SetFont(wx.Font( 14, 74, 90, 90, False, "Calibri" ) )

self.F_D_disp_textCtrl_6.SetBackgroundColour( wx.SystemSettings.GetColour(wx.SYS_COLOUR_SCROLLBAR ) )

force_disp_indentation_parm_6.Add( self.F_D_disp_textCtrl_6, wx.GBPosition( 3,1 ), wx.GBSpan $(1,1)$,

wx.BOTTOM|wx.LEFT|wx.RIGHT|wx.ALIGN_CENTER_HORIZONTAL, 5 )

self.F_D_vel_textCtrl_6 =wx.TextCtrl( self.Force_Displacement, wx.ID_ANY, wx.EmptyString, wx.DefaultPosition, wx.Size( 90,-1 ),

wx.TE_RIGHT|wx.TE_READONLY)

self.F_D_vel_textCtrl_6.SetFont( wx.Font( 14, 74, 90, 90, False, "Calibri" ) )

self.F_D_vel_textCtrl_6.SetBackgroundColour(wx.SystemSettings.GetColour(wx.SYS_COLOUR_SCROLLBAR ) )

force_disp_indentation_parm_6.Add( self.F_D_vel_textCtrl_6, wx.GBPosition $(3,2$ ), wx.GBSpan( 1,1 ),

wx.BOTTOM|wx.LEFT|wx.RIGHT|wx.ALIGN_CENTER_HORIZONTAL, 5 )

\#self.max_dispText12 = wx.StaticText( self.Force_Displacement, wx.ID_ANY, u"5", wx.DefaultPosition, wx.DefaultSize, wx.ALIGN_RIGHT )

\#self.max_dispText12.Wrap (-1)

\#self.max_dispText12.SetFont( wx.Font( 12, 74, 90, 90, False, "Calibri" ) )

\#self.max_dispText12.SetForegroundColour(wx.SystemSettings.GetColour(wx.SYS_COLOUR_SCROLLBAR ) )

\#self.max_dispText12.SetMinSize( wx.Size( $100,-1$ ) )

\#force_disp_indentation_parm_6.Add( self.max_dispText12,wx.GBPosition( 3, 2), wx.GBSpan( 1, 1 ),

wx.ALL/wx.ALIGN_CENTER_HORIZONTAL|wx.ALIGN_CENTER_VERTICAL, 5 )

self.vel_text12 = wx.StaticText( self.Force_Displacement, wx.ID_ANY, u"velocity (mm/s)", wx.DefaultPosition, wx.DefaultSize,

wx.ALIGN_LEFT )

self.vel_text12.Wrap $(-1)$

self.vel_text12.SetFont( wx.Font( 12, 74, 90, 90, False, "Calibri" ) )

force_disp_indentation_parm_6.Add( self.vel_text12, wx.GBPosition( 2, 2), wx.GBSpan( 1, 1 ),

wx.ALIGN_BOTTOM|wx.LEFT|wx.RIGHT|wx.TOP|wx.ALIGN_LEFT, 5 )

self.acc_text12 $=$ wx.StaticText( self.Force_Displacement, wx.ID_ANY, u"displ. (mm)", wx.DefaultPosition, wx.DefaultSize, wx.ALIGN_LEFT )

self.acc_text12.Wrap( -1$)$

self.acc_text12.SetFont( wx.Font( 12, 74, 90, 90, False, "Calibri" ) )

force_disp_indentation_parm_6.Add( self.acc_text12, wx.GBPosition( 2,1 ), wx.GBSpan $(1,1)$,

wx.TOP|wx.RIGHT|wx.LEFT|wx.ALIGN_LEFT|wx.ALIGN_BOTTOM, 5 )

\#self.maxVel_text12 = wx.StaticText( self.Force_Displacement, wx.ID_ANY, u"40", wx.DefaultPosition, wx.DefaultSize, 0 ) 
\#self.maxVel_text12.Wrap ( -1 )

\#self.maxVel_text12.SetFont( wx.Font( 12, 74, 90, 90, False, "Calibri" ) )

\#self.maxVel_text12.SetForegroundColour( wx.SystemSettings.GetColour(wx.SYS_COLOUR_SCROLLBAR ) )

\#force_disp_indentation_parm_6.Add( self.maxVel_text12, wx.GBPosition( 5, 2 ), wx.GBSpan ( 1,1 ),

wx.ALL/wx.ALIGN_CENTER_HORIZONTAL|wx.ALIGN_CENTER_VERTICAL, 5 )

\#self.min_Acc_text12 $=$ wx.StaticText( self.Force_Displacement, wx.ID_ANY, u"1.0", wx.DefaultPosition, wx.DefaultSize, 0 )

\#self.min_Acc_text12.Wrap( -1$)$

\#self.min_Acc_text12.SetFont( wx.Font( 12, 74, 90, 90, False, "Calibri" ) )

\#self.min_Acc_text12.SetForegroundColour(wx.SystemSettings.GetColour(wx.SYS_COLOUR_SCROLLBAR ) )

\#force_disp_indentation_parm_6.Add( self.min_Acc_text12, wx.GBPosition( 7,0 ), wx.GBSpan( 1,1 ),

wx.ALIGN_CENTER_HORIZONTAL|wx.TOP|wx.BOTTOM|wx.LEFT|wx.ALIGN_CENTER_VERTICAL, 5 )

\#self.max_Acc_text12 = wx.StaticText( self.Force_Displacement, wx.ID_ANY, u"5", wx.DefaultPosition, wx.DefaultSize, 0 )

\#self.max_Acc_text12.Wrap( -1 )

\#self.max_Acc_text12.SetFont( wx.Font( 12, 74, 90, 90, False, "Calibri" ) )

\#self.max_Acc_text12.SetForegroundColour(wx.SystemSettings.GetColour( wx.SYS_COLOUR_SCROLLBAR ) )

\#force_disp_indentation_parm_6.Add( self.max_Acc_text12, wx.GBPosition( 7,2 ), wx.GBSpan( 1,1 ),

wx.ALL|wx.ALIGN_CENTER_HORIZONTAL|wx.ALIGN_CENTER_VERTICAL, 5 )

self.hold_text12 $=w$ w.StaticText $($ self.Force_Displacement, wx.ID_ANY, u"hold (s) ", wx.DefaultPosition, wx.DefaultSize, wx.ALIGN_LEFT ) self.hold_text12.Wrap( -1 )

self.hold_text12.SetFont( wx.Font( 12, 74, 90, 90, False, "Calibri" ) )

force_disp_indentation_parm_6.Add( self.hold_text12, wx.GBPosition( 4, 1 ), wx.GBSpan( 1,1 ),

wx.TOP|wx.RIGHT|wx.LEFT|wx.ALIGN_CENTER_VERTICAL|wx.ALIGN_LEFT, 5 )

self.min_holdText12 = wx.StaticText( self.Force_Displacement, wx.ID_ANY, u"1.5", wx.DefaultPosition, wx.DefaultSize, wx.ALIGN_LEFT )

self.min_holdText12.Wrap $(-1)$

self.min_holdText12.SetFont( wx.Font( 12, 74, 90, 90, False, "Calibri" ) )

self.min_holdText12.SetForegroundColour( $(96,96,96))$

self.min_holdText12.SetMinSize( wx.Size $(100,-1)$ )

force_disp_indentation_parm_6.Add( self.min_holdText12,wx.GBPosition( 5, 0 ), wx.GBSpan( 1,1 ),

wx.ALIGN_CENTER_HORIZONTAL/wx.TOP|wx.BOTTOM|wx.LEFT|wx.ALIGN_CENTER_VERTICAL, 5 )

self.F_D_holdTime_6 $=$ FS.FloatSpin( self.Force_Displacement, -1 , value $=5, \min \_$val $=1.5$, max_val $=600$, increment $=$

.5 ,agwStyle=FS.FS_RIGHT)

self.F_D_holdTime_6.SetFormat("\%f")

self.F_D_holdTime_6.SetDigits(2)

self.F_D_holdTime_6.SetFont( wx.Font ( 12, 74, 90, 90, False, "Calibri" ) )

self.F_D_holdTime_6.SetForegroundColour( wx.SystemSettings.GetColour( wx.SYS_COLOUR_BTNTEXT ) )

self.F_D_holdTime_6.SetBackgroundColour(wx.SystemSettings.GetColour( wx.SYS_COLOUR_BTNSHADOW ) )

force_disp_indentation_parm_6.Add( self.F_D_holdTime_6, wx.GBPosition $(5,1)$, wx.GBSpan $(1,1)$,

wx.ALIGN_CENTER_HORIZONTAL|wx.BOTTOM|wx.RIGHT|wx.LEFT, 5 )

self.maxHold_text12 = wx.StaticText( self.Force_Displacement, wx.ID_ANY, u"600", wx.DefaultPosition, wx.DefaultSize, wx.ALIGN_RIGHT )

self.maxHold_text12.Wrap( -1 )

self.maxHold_text12.SetFont( wx.Font( 12, 74, 90, 90, False, "Calibri" ) )

self.maxHold_text12.SetForegroundColour $((96,96,96))$

self.maxHold_text12.SetMinSize (wx.Size $(100,-1))$

force_disp_indentation_parm_6.Add( self.maxHold_text12,wx.GBPosition( 5, 2 ), wx.GBSpan( 1, 1 ),

wx.ALL|wx.ALIGN_CENTER_HORIZONTAL|wx.ALIGN_CENTER_VERTICAL, 5 )

self.thickness_text12 $=$ wx.StaticText( self.Force_Displacement, wx.ID_ANY, u"thickness", wx.DefaultPosition, wx.DefaultSize, wx.ALIGN_LEFT )

self.thickness_text12.Wrap( -1 )

self.thickness_text12.SetFont( wx.Font( 12, 74, 90, 90, False, "Calibri" ) )

force_disp_indentation_parm_6.Add( self.thickness_text12, wx.GBPosition( 6, 1), wx.GBSpan( 1, 1 ),

wx.TOP|wx.RIGHT|wx.LEFT|wx.ALIGN_CENTER_VERTICAL|wx.ALIGN_LEFT, 5 )

self.min_thicknessText12 = wx.StaticText( self.Force_Displacement, wx.ID_ANY, u"sylguard", wx.DefaultPosition, wx.DefaultSize, wX.ALIGN_LEFT)

self.min_thicknessText12.Wrap( -1 )

self.min_thicknessText12.SetFont( wx.Font (12, 74, 90, 90, False, "Calibri" ) )

self.min_thicknessText12.SetForegroundColour((96,96,96))

self.min_thicknessText12.SetMinSize( wx.Size $(100,-1)$ )

force_disp_indentation_parm_6.Add( self.min_thicknessText12, wx.GBPosition( 7, 0 ), wx.GBSpan( 1, 1 ),

wx.ALIGN_CENTER_HORIZONTAL|wx.TOP|wx.BOTTOM|wx.LEFT|wx.ALIGN_CENTER_VERTICAL, 5 )

self.F_D_skinThickness $=$ wx.Slider( self.Force_Displacement, $-1,0,-10,10$,style $=$ wx.SL_HORIZONTAL $\mid$ wx.SL_AUTOTICKS $)$

force_disp_indentation_parm_6.Add( self.F_D_skinThickness, wx.GBPosition $(7,1)$, wx.GBSpan $(1,1)$,

wx.ALIGN_CENTER_HORIZONTAL|wx.BOTTOM|wx.RIGHT|wx.LEFT, 5 )

self.F_D_default_button = wx.Button( self.Force_Displacement, 100, u"Default", wx.DefaultPosition, wx.Size ( 100,30 ), 0 )

self.F_D_default_button.SetFont( wx.Font( 14, 74, 90, 92, False, "Calibri" ) )

self.Bind(wx.EVT_BUTTON,self.restoreDefaults,id=100) 
force_disp_indentation_parm_6.Add( self.F_D_default_button, wx.GBPosition( 8,1 ), wx.GBSpan $(1,1)$, wx.ALIGN_CENTER_HORIZONTAL|wx.BOTTOM|wx.RIGHT|wx.LEFT, 5 )

self.maxthick_text12 $=$ wx.StaticText( self.Force_Displacement, wx.ID_ANY, u"thick", wx.DefaultPosition, wx.DefaultSize, wx.ALIGN_RIGHT)

self.maxthick_text12.Wrap( -1 )

self.maxthick_text12.SetFont( wx.Font( 12, 74, 90, 90, False, "Calibri" ) )

self.maxthick_text12.SetForegroundColour $((96,96,96))$

self.maxthick_text12.SetMinSize( wx.Size $(100,-1)$ )

force_disp_indentation_parm_6.Add( self.maxthick_text12, wx.GBPosition( 7, 2), wx.GBSpan( 1,1 ),

wx.ALL|wx.ALIGN_CENTER_HORIZONTAL|wx.ALIGN_CENTER_VERTICAL, 5 )

F_D_ID_6Choices $=$ []

self.F_D_ID_6 = wx.Choice( self.Force_Displacement, 24000, wx.DefaultPosition, wx.DefaultSize, F_D_ID_6Choices, 0 )

self.F_D_ID_6.SetSelection( 0 )

force_disp_indentation_parm_6.Add( self.F_D_ID_6, wx.GBPosition( 1, 1 ), wx.GBSpan( 1, 1 ),

wx.ALIGN_CENTER_HORIZONTAL/wx.RIGHT|wx.LEFT, 5 )

self.Bind(wx.EVT_CHOICE,self.chosenID,id=24000)

self.m_staticText1271 = wx.StaticText( self.Force_Displacement, wx.ID_ANY, u"ID", wx.DefaultPosition, wx.DefaultSize, 0 )

self.m_staticText1271.Wrap( -1 )

self.m_staticText1271.SetFont( wx.Font( 12, 74, 90, 90, False, "Calibri" ) )

force_disp_indentation_parm_6.Add( self.m_staticText1271, wx.GBPosition( 0,1 ), wx.GBSpan( 1,1 ),

wx.ALIGN_CENTER_HORIZONTAL|wx.ALIGN_BOTTOM|wx.RIGHT|wx.LEFT, 5 )

Force_Disp_tab_sizer_6.Add( force_disp_indentation_parm_6, wx.GBPosition( 1,0 ), wx.GBSpan $(1,1)$,

wx.ALIGN_CENTER_HORIZONTAL|wx.ALIGN_CENTER_VERTICAL, 5 )

F_D_indentButton_sizer_6 = wx.BoxSizer(wx.VERTICAL )

self.F_D_Indent_button = wx.Button( self.Force_Displacement, 25, u"Indent", wx.DefaultPosition, wx.Size $(175,60$ ), 0 )

self.F_D_Indent button.SetFont( wx.Font( 18, 74, 90, 92, False, "Calibri" ))

self.Bind(wx.EVT_BUTTON,self.runIndent_FD_6,id=25)

F_D_indentButton_sizer_6.Add( self.F_D_Indent_button, 0, wx.ALIGN_CENTER_HORIZONTAL|wx.RIGHT|wx.LEFT, 5 )

Force_Disp_tab_sizer_6.Add( F_D_indentButton_sizer_6, wx.GBPosition( 2, 0 ), wx.GBSpan( 1,1 ), wx.EXPAND, 5 )

self.m_panel $80=$ wx.Panel( self.Force_Displacement, wx.ID_ANY, wx.DefaultPosition, wx.DefaultSize,

wx.TAB_TRAVERSAL|wx.SIMPLE_BORDER )

bSizer57 $=$ wx.BoxSizer $(w x$. VERTICAL $)$

self.levels_calibrate_text_6 $=$ wx.StaticText( self.m_panel80,wx.ID_ANY, u"Force levels calibrated", wx.DefaultPosition, wx.DefaultSize, 0 ) self.levels_calibrate_text_6.Wrap( -1 )

self.levels_calibrate_text_6.SetFont( wx.Font( 14, 74, 90, 92, False, "Calibri" ) )

bSizer57.Add( self.levels_calibrate_text_6, $0,0,5$ )

self.F_D_table_panel_6 = wx.ScrolledWindow( self.m_panel80, wx.ID_ANY, wx.DefaultPosition, $(360,300)$,

wx.HSCROLL|wx.SIMPLE_BORDER|wx.VSCROLL )

self.F_D_table_panel_6.SetScrollRate( 5,5 )

F_D_table_sizer_6 = wx.FlexGridSizer $(0,7,0,0)$

F_D_table_sizer_6.SetFlexibleDirection(wx.BOTH )

F_D_table_sizer_6.SetNonFlexibleGrowMode( wx.FLEX_GROWMODE_SPECIFIED )

self.F_D_table_panel_6.SetMinSize( wx.Size( 360,300))

self.F_D_ID_panel_6 = wx.Panel( self.F_D_table_panel_6, wx.ID_ANY, wx.DefaultPosition, wx.DefaultSize, wx.TAB_TRAVERSAL )

self.F_D_ID_panel_6.SetBackgroundColour( wx.SystemSettings.GetColour( wx.SYS_COLOUR_SCROLLBAR ) )

self.F_D_ID_panel_6.SetMinSize( wx.Size $(-1,700))$

self.F_D_ID_sizer_6 = wx.BoxSizer( wx.VERTICAL )

self.F_D_ID_sizer_6.SetMinSize( wx.Size $(-1,700)$ )

self.F_D_ID_header_6 $=$ wx.StaticText( self.F_D_ID_panel_6, wx.ID_ANY, u"ID", wx.DefaultPosition, wx.Size( 50,30 ),

wX.ALIGN_CENTRE)

self.F_D_ID_header_6.Wrap( -1 )

self.F_D_ID_header_6.SetBackgroundColour(wx.SystemSettings.GetColour(wx.SYS_COLOUR_SCROLLBAR ) )

self.F_D_ID_sizer_6.Add( self.F_D_ID_header_6, 0, wx.ALL, 5 )

self.type_staticline1 $=$ wx.StaticLine( self.F_D_ID_panel_6, wx.ID_ANY, wx.DefaultPosition, wx.DefaultSize, wx.LI_HORIZONTAL )

self.F_D_ID_sizer_6.Add( self.type_staticline1, 0, wx.EXPAND|wx.TOP|wx.BOTTOM, 5 )

self.F_D_ID_panel_6.SetSizer( self.F_D_ID_sizer_6 )

self.F_D_ID_panel_6.Layout()

self.F_D_ID_sizer_6.Fit( self.F_D_ID_panel_6)

F_D_table_sizer_6.Add( self.F_D_ID_panel_6, 1, wx.EXPAND|wx.BOTTOM, 5 )

self.F_D_force_panel_6 = wx.Panel( self.F_D_table_panel_6, wx.ID_ANY, wx.DefaultPosition, wx.DefaultSize, wx.TAB_TRAVERSAL )

self.F_D_force_panel_6.SetBackgroundColour(wx.SystemSettings.GetColour(wx.SYS_COLOUR_3DLIGHT ) )

self.F_D_force_panel_6.SetMinSize (wx.Size (100,700 ) )

self.F_D_force_sizer_6 = wx.BoxSizer(wx.VERTICAL )

self.F_D_force_sizer_6.SetMinSize( wx.Size (100,700 ) ) 
self.F_D_Force_header_6 $=$ wx.StaticText( self.F_D_force_panel_6, wx.ID_ANY, u"force $\backslash n(m N) "$, wx.DefaultPosition, wx.Size $(-1,30)$, wx.ALIGN_CENTRE )

self.F_D_Force_header_6.Wrap ( -1 )

self.F_D_force_sizer_6.Add( self.F_D_Force_header_6, 0, wx.ALL|wx.ALIGN_CENTER_HORIZONTAL, 5 )

self.time_staticline1 $=$ wx.StaticLine( self.F_D_force_panel_6, wx.ID_ANY, wx.DefaultPosition, wx.DefaultSize, wx.LI_HORIZONTAL )

self.F_D_force_sizer_6.Add( self.time_staticline1, 0, wx.EXPAND|wx.TOP|wx.BOTTOM, 5 )

self.F_D_force_panel_6.SetSizer( self.F_D_force_sizer_6 )

self.F_D_force_panel_6.Layout()

self.F_D_force_sizer_6.Fit( self.F_D_force_panel_6)

F_D_table_sizer_6.Add( self.F_D_force_panel_6, 1, wx.EXPAND|wx.BOTTOM, 5 )

self.F_D_disp_panel_6 $=$ wx.Panel( self.F_D_table_panel_6, wx.ID_ANY, wx.DefaultPosition, wx.DefaultSize, wx.TAB_TRAVERSAL )

self.F_D_disp_panel_6.SetBackgroundColour(wx.SystemSettings.GetColour(wx.SYS_COLOUR_SCROLLBAR ) )

self.F_D_disp_panel_6.SetMinSize ( wx.Size $(-1,700))$

self.F_D_disp_sizer_6 = wx.BoxSizer( wx.VERTICAL )

self.F_D_disp_sizer_6.SetMinSize( wx.Size (100,700 ) )

self.F_D_disp_header_6 = wx.StaticText( self.F_D_disp_panel_6, wx.ID_ANY, u"displacement $\ln (\mathrm{mm})$ ", wx.DefaultPosition, wx.Size( $-1,30$ ), wX.ALIGN_CENTRE)

self.F_D_disp_header_6.Wrap( -1 )

self.F_D_disp_sizer_6.Add( self.F_D_disp_header_6, 0, wx.ALL|wx.ALIGN_CENTER_HORIZONTAL, 5 )

self.disp_staticline1 $=$ wx.StaticLine $($ self.F_D_disp_panel_6, wx.ID_ANY, wx.DefaultPosition, wx.DefaultSize, wx.LI_HORIZONTAL )

self.F_D_disp_sizer_6.Add( self.disp_staticline1, 0, wx.EXPAND|wx.TOP|wx.BOTTOM, 5 )

self.F_D_disp_panel_6.SetSizer( self.F_D_disp_sizer_6 )

self.F_D_disp_panel_6.Layout()

self.F_D_disp_sizer_6.Fit( self.F_D_disp_panel_6)

F_D_table_sizer_6.Add( self.F_D_disp_panel_6, 1, wx.EXPAND|wx.BOTTOM, 5 )

self.F_D_vel_panel_6 $=$ wx.Panel( self.F_D_table_panel_6, wx.ID_ANY, wx.DefaultPosition, wx.DefaultSize, wx.TAB_TRAVERSAL )

self.F_D_vel_panel_6.SetMinSize $($ wx.Size $(-1,700))$

self.F_D_vel_sizer_6 = wx.BoxSizer( wx.VERTICAL )

self.F_D_vel_sizer_6.SetMinSize( wx.Size( 100,700))

self.F_D_vel_header_6 $=$ wx.StaticText( self.F_D_vel_panel_6, wx.ID_ANY, u"velocity $\backslash n(\mathrm{~mm} / \mathrm{s}) "$ ", wx.DefaultPosition, wx.Size ( 50,30 ),

wx.ALIGN_CENTRE )

self.F_D_vel_header_6.Wrap( -1 )

self.F_D_vel_sizer_6.Add( self.F_D_vel_header_6, 0, wx.ALL|wx.ALIGN_CENTER_HORIZONTAL, 5 )

self.vel_staticline1 = wx.StaticLine ( self.F_D_vel_panel_6, wx.ID_ANY, wx.DefaultPosition, wx.DefaultSize, wx.LI_HORIZONTAL )

self.F_D_vel_sizer_6.Add( self.vel_staticline 1, 0, wx.EXPAND|wx.TOP|wx.BOTTOM, 5 )

self.F_D_vel_panel_6.SetSizer( self.F_D_vel_sizer_6 )

self.F_D_vel_panel_6.Layout()

self.F_D_vel_sizer_6.Fit( self.F_D_vel_panel_6)

F_D_table_sizer_6.Add( self.F_D_vel_panel_6, 1, wx.EXPAND|wx.BOTTOM, 5 )

self.F_D_table_panel_6.SetSizer( F_D_table_sizer_6 )

self.F_D_table_panel_6.Layout()

F_D_table_sizer_6.Fit( self.F_D_table_panel_6 )

bSizer57.Add( self.F_D_table_panel_6, 1, wx.ALL|wx.ALIGN_CENTER_HORIZONTAL, 5 )

self.m_panel80.SetSizer( bSizer57 )

self.m_panel80.Layout()

bSizer57.Fit( self.m_panel80)

Force_Disp_tab_sizer_6.Add( self.m_panel80,wx.GBPosition( 3, 0 ), wx.GBSpan ( 1, 1 ), wx.EXPAND, 5 )

self.Force_Displacement.SetSizer( Force_Disp_tab_sizer_6 )

self.Force_Displacement.Layout()

Force_Disp_tab_sizer_6.Fit( self.Force_Displacement)

self.step6_force_panel.AddPage( self.Force_Displacement, u"Force and Displacement", True )

self.fineDisp_step6 = wx.Panel( self.step6_force_panel, wx.ID_ANY, wx.DefaultPosition, wx.DefaultSize, wx.TAB_TRAVERSAL )

fineDisp_sizer_step6 $=$ wx.FlexGridSizer $(0,2,0,0)$

fineDisp_sizer_step6.SetFlexibleDirection(wx.BOTH )

fineDisp_sizer_step6.SetNonFlexibleGrowMode(wx.FLEX_GROWMODE_SPECIFIED )

self.fineDisp_panel_6 = wx.Panel( self.fineDisp_step6, wx.ID_ANY, wx.DefaultPosition, wx.Size( 660,350 ), wx.SIMPLE_BORDER )

self.fineDisp_panel_6.SetMinSize (wx.Size $(-1,600)$ )

fineDisp_sizer_6 $=$ wx.FlexGridSizer $(5,1,0,0)$

fineDisp_sizer_6.SetFlexibleDirection( wx.BOTH )

fineDisp_sizer_6.SetNonFlexibleGrowMode( wx.FLEX_GROWMODE_SPECIFIED )

fine_indentparm_text_sizer_6 $=$ wx.FlexGridSizer $(0,2,0,0)$

fine_indentparm_text_sizer_6.SetFlexibleDirection( wx.HORIZONTAL )

fine_indentparm_text_sizer_6.SetNonFlexibleGrowMode( wx.FLEX_GROWMODE_SPECIFIED )

fine_indentparm_text_sizer_6.SetMinSize( wx.Size $(660,-1)$ )

self.fine_indent_text_6 =wx.StaticText( self.fineDisp_panel_6, wx.ID_ANY, u"Indentation parameters", wx.DefaultPosition, wx.DefaultSize, 0 )

self.fine_indent_text_6.Wrap (-1)

self.fine_indent_text_6.SetFont( wx.Font (14, 74, 90, 92, False, "Calibri" ) )

fine_indentparm_text_sizer_6.Add( self.fine_indent_text_6, 0,wx.ALL, 5 ) 
fineDisp_sizer_6.Add( fine_indentparm_text_sizer_6, 1, wx.EXPAND, 5 )

fine_parm_sizer_6 $=$ wx.GridBagSizer $(0,0)$

fine_parm_sizer_6.SetFlexibleDirection(wx.BOTH )

fine_parm_sizer_6.SetNonFlexibleGrowMode( wx.FLEX_GROWMODE_SPECIFIED )

self.max_text $=$ wx.StaticText( self.fineDisp_panel_6, wx.ID_ANY, u"Max", wx.DefaultPosition, wx.DefaultSize, 0 )

self.max_text.Wrap $(-1)$

self.max_text.SetFont( wx.Font( 12, 74, 90, 90, False, "Calibri" ) )

self.max_text.SetForegroundColour( wx.SystemSettings.GetColour(wx.SYS_COLOUR_SCROLLBAR ) )

fine_parm_sizer_6.Add( self.max_text, wx.GBPosition( 0,2), wx.GBSpan $(1,1)$,

wx.ALIGN_RIGHT|wx.ALIGN_CENTER_VERTICAL|wx.LEFT|wx.TOP|wx.BOTTOM, 5 )

self.displacement_text $=$ wx.StaticText( self.fineDisp_panel_6, wx.ID_ANY, u"displ. (mm)", wx.DefaultPosition, wx.DefaultSize, wx.ALIGN_CENTRE )

self.displacement_text.Wrap( -1 )

self.displacement_text.SetFont( wx.Font( 12, 74, 90, 90, False, "Calibri" ) )

fine_parm_sizer_6.Add( self.displacement_text, wx.GBPosition( 0, 1 ), wx.GBSpan( 1, 1 ), wx.LEFT|wx.RIGHT|wx.ALIGN_BOTTOM, 5 )

self.min_text $=$ wx.StaticText $($ self.fineDisp_panel_6, wx.ID_ANY, u"Min", wx.DefaultPosition, wx.DefaultSize, wx.ALIGN_LEFT)

self.min_text.Wrap( -1 )

self.min_text.SetFont( wx.Font( 12, 74, 90, 90, False, "Calibri" ) )

self.min_text.SetForegroundColour( wx.SystemSettings.GetColour(wx.SYS_COLOUR_SCROLLBAR ))

fine_parm_sizer_6.Add( self.min_text, wx.GBPosition( 0,0$)$, wx.GBSpan $(1,1)$,

wx.TOP|wx.BOTTOM|wx.RIGHT|wx.ALIGN_LEFT|wx.ALIGN_CENTER_VERTICAL, 5 )

self.minDisp_text $=$ wx.StaticText $($ self.fineDisp_panel_6, wx.ID_ANY, u"0.01", wx.DefaultPosition, wx.Size ( $-1,-1$ ), wx.ALIGN_LEFT )

self.minDisp_text.Wrap ( -1$)$

self.minDisp_text.SetFont( wx.Font( 12, 74, 90, 90, False, "Calibri" ) )

self.minDisp_text.SetForegroundColour(wx.SystemSettings.GetColour(wx.SYS_COLOUR_SCROLLBAR ) )

self.minDisp_text.SetMinSize( wx.Size $(100,-1))$

fine_parm_sizer_6.Add( self.minDisp_text, wx.GBPosition( 1,0), wx.GBSpan( 1,1 ),

wx.ALIGN_LEFT|wx.RIGHT|wx.TOP|wx.BOTTOM|wx.ALIGN_CENTER_VERTICAL, 5 )

self.fine_disp_6 $=$ FS.FloatSpin ( self.fineDisp_panel_6, - 1 , min_val $=.01, \max \_v a l=5$,increment $=.01$, agwStyle=FS.FS_RIGHT )

self.fine_disp_6.SetFormat("\%f")

self.fine_disp_6.SetDigits(2)

self.fine_disp_6.SetFont( wx.Font( 12, 74, 90, 90, False, "Calibri" ) )

self.fine_disp_6.SetForegroundColour(wx.SystemSettings.GetColour( wx.SYS_COLOUR_BTNTEXT ) )

self.fine_disp_6.SetBackgroundColour(wx.SystemSettings.GetColour(wx.SYS_COLOUR_BTNSHADOW ) )

fine_parm_sizer_6.Add( self.fine_disp_6, wx.GBPosition( 1, 1), wx.GBSpan( 1,1 ),

wx.BOTTOM|wx.RIGHT|wx.LEFT|wx.ALIGN_CENTER_HORIZONTAL, 5 )

self.minVel_text $=$ wx.StaticText $($ self.fineDisp_panel_6, wx.ID_ANY, u"1.0", wx.DefaultPosition, wx.Size ( -1,-1 ), wx.ALIGN_CENTRE )

self.minVel_text.Wrap( -1$)$

self.minVel_text.SetFont(wx.Font( 12, 74, 90, 90, False, "Calibri" ) )

self.minVel_text.SetForegroundColour( wx.SystemSettings.GetColour( wx.SYS_COLOUR_SCROLLBAR ) )

fine_parm_sizer_6.Add( self.minVel_text, wx.GBPosition( 3,0), wx.GBSpan $(1,1)$,

wx.ALIGN_LEFT|wx.TOP|wx.BOTTOM|wx.RIGHT|wx.ALIGN_CENTER_VERTICAL, 5 )

self.max_dispText $=$ wx.StaticText( self.fineDisp_panel_6, wx.ID_ANY, u"5", wx.DefaultPosition, wx.DefaultSize, wx.ALIGN_RIGHT )

self.max_dispText.Wrap( -1$)$

self.max_dispText.SetFont( wx.Font( 12, 74, 90, 90, False, "Calibri" ) )

self.max_dispText.SetForegroundColour( wx.SystemSettings.GetColour( wx.SYS_COLOUR_SCROLLBAR ) )

self.max_dispText.SetMinSize( wx.Size( 100,-1 ))

fine_parm_sizer_6.Add( self.max_dispText, wx.GBPosition( 1,2 ), wx.GBSpan( 1,1),

wx.TOP|wx.BOTTOM|wx.LEFT|wx.ALIGN_RIGHT|wx.ALIGN_CENTER_VERTICAL, 5 )

self.vel_text $=$ wx.StaticText( self.fineDisp_panel_6, wx.ID_ANY, u"velocity $(\mathrm{mm} / \mathrm{s}) "$, wx.DefaultPosition, wx.DefaultSize,

wx.ALIGN_CENTRE )

self.vel_text.Wrap( -1$)$

self.vel_text.SetFont( wx.Font( 12, 74, 90, 90, False, "Calibri" ) )

fine_parm_sizer_6.Add( self.vel_text, wx.GBPosition( 2,1 ), wx.GBSpan( 1,1 ), wx.ALIGN_BOTTOM|wx.LEFT|wx.RIGHT|wx.TOP, 5 )

self.fine_vel_6 $=$ FS.FloatSpin( self.fineDisp_panel_6, -1, min_val=1, max_val=40.0, increment $=.1$, agwStyle=FS.FS_RIGHT )

self.fine_vel_6.SetFormat("\%f")

self.fine_vel_6.SetDigits(2)

self.fine_vel_6.SetFont( wx.Font( 12, 74, 90, 90, False, "Calibri" ) )

self.fine_vel_6.SetForegroundColour( wx.SystemSettings.GetColour(wx.SYS_COLOUR_BTNTEXT ) )

self.fine_vel_6.SetBackgroundColour( wx.SystemSettings.GetColour(wx.SYS_COLOUR_BTNSHADOW ) )

fine_parm_sizer_6.Add( self.fine_vel_6, wx.GBPosition( 3,1), wx.GBSpan( 1,1 ),

wx.BOTTOM $\mid$ wx.RIGHT|wx.LEFT|wx.ALIGN_CENTER_HORIZONTAL, 5 )

self.maxVel_text $=$ wx.StaticText( self.fineDisp_panel_6, wx.ID_ANY, u"40", wx.DefaultPosition, wx.DefaultSize, 0 )

self.maxVel_text.Wrap( -1$)$

self.maxVel_text.SetFont(wx.Font( 12, 74, 90, 90, False, "Calibri" ) )

self.maxVel_text.SetForegroundColour(wx.SystemSettings.GetColour(wx.SYS_COLOUR_SCROLLBAR ) )

fine_parm_sizer_6.Add( self.maxVel_text, wx.GBPosition( 3, 2), wx.GBSpan( 1,1 ),

wx.LEFT|wx.TOP|wx.BOTTOM|wx.ALIGN_RIGHT|wx.ALIGN_CENTER_VERTICAL, 5 ) 
self.acc_text $=$ wx.StaticText $($ self.fineDisp_panel_6, wx.ID_ANY, u"accel $(\mathrm{mm} / \mathrm{s} / \mathrm{s}) "$, wx.DefaultPosition, wx.DefaultSize, wx.ALIGN_CENTRE )

self.acc_text.Wrap $(-1)$

self.acc_text.SetFont( wx.Font( 12, 74, 90, 90, False, "Calibri" ) )

fine_parm_sizer_6.Add( self.acc_text, wx.GBPosition( 4,1 ), wx.GBSpan( 1,1 ), wx.TOP|wx.RIGHT|wx.LEFT, 5 )

self.min_Acc_text $=$ wx.StaticText $($ self.fineDisp_panel_6, wx.ID_ANY, u"1.0", wx.DefaultPosition, wx.DefaultSize, 0 )

self.min_Acc_text.Wrap( -1 )

self.min_Acc_text.SetFont(wx.Font( 12, 74, 90, 90, False, "Calibri" ) )

self.min_Acc_text.SetForegroundColour( wx.SystemSettings.GetColour(wx.SYS_COLOUR_SCROLLBAR ) )

fine_parm_sizer_6.Add( self.min_Acc_text, wx.GBPosition( 5, 0 ), wx.GBSpan $(1,1$ ),

wx.ALIGN_LEFT|wx.TOP|wx.BOTTOM|wx.RIGHT|wx.ALIGN_CENTER_VERTICAL, 5 )

self.fine_acc_6 $=$ FS.FloatSpin( self.fineDisp_panel_6, -1 , min_val=1, max_val=160, increment $=1$, agwStyle=FS.FS_RIGHT)

self.fine_acc_6.SetFormat("\%f")

self.fine_acc_6.SetDigits(2)

self.fine_acc_6.SetFont( wx.Font( 12, 74, 90, 90, False, "Calibri" ) )

self.fine_acc_6.SetForegroundColour( wx.SystemSettings.GetColour(wx.SYS_COLOUR_BTNTEXT ) )

self.fine_acc_6.SetBackgroundColour( wx.SystemSettings.GetColour( wx.SYS_COLOUR_BTNSHADOW ) )

fine_parm_sizer_6.Add( self.fine_acc_6, wx.GBPosition( 5, 1), wx.GBSpan $(1,1)$,

wx.BOTTOM|wx.RIGHT|wx.LEFT|wx.ALIGN_CENTER_HORIZONTAL, 5 )

self.max_Acc_text $=$ wx.StaticText( self.fineDisp_panel_6, wx.ID_ANY, u"160", wx.DefaultPosition, wx.DefaultSize, 0 )

self.max_Acc_text.Wrap( -1 )

self.max_Acc_text.SetFont( wx.Font (12, 74, 90, 90, False, "Calibri" ) )

self.max_Acc_text.SetForegroundColour(wx.SystemSettings.GetColour(wx.SYS_COLOUR_SCROLLBAR ) )

fine_parm_sizer_6.Add( self.max_Acc_text, wx.GBPosition( 5, 2), wx.GBSpan $(1,1)$,

wx.TOP|wx.LEFT|wx.BOTTOM|wx.ALIGN_RIGHT|wx.ALIGN_CENTER_VERTICAL, 5 )

self.hold_text $=$ wx.StaticText ( self.fineDisp_panel_6, wx.ID_ANY, u"hold (s) ", wx.DefaultPosition, wx.DefaultSize, wx.ALIGN_CENTRE ) self.hold_text.Wrap $(-1)$

self.hold_text.SetFont(wx.Font( 12, 74, 90, 90, False, "Calibri" ) )

fine_parm_sizer_6.Add( self.hold_text, wx.GBPosition( 6,1 ), wx.GBSpan( 1,1 ),

wx.TOP|wx.RIGHT|wx.LEFT|wx.ALIGN_CENTER_VERTICAL|wx.ALIGN_LEFT, 5 )

self.min_holdText = wx.StaticText( self.fineDisp_panel_6, wx.ID_ANY, u"0.1", wx.DefaultPosition, wx.DefaultSize, wx.ALIGN_CENTRE )

self.min_holdText.Wrap ( -1 )

self.min_holdText.SetFont( wx.Font( 12, 74, 90, 90, False, "Calibri" ))

self.min_holdText.SetForegroundColour(wx.SystemSettings.GetColour( wx.SYS_COLOUR_SCROLLBAR ) )

fine_parm_sizer_6.Add( self.min_holdText, wx.GBPosition( 7, 0 ), wx.GBSpan( 1,1 ),

wx.ALIGN_LEFT|wx.TOP|wx.BOTTOM|wx.LEFT|wx.ALIGN_CENTER_VERTICAL, 5 )

self.fine_hold_6 $=$ FS.FloatSpin( $\operatorname{self}$. fineDisp_panel_6, $-1, \min \_$val $=.1$, max_val=600,increment $=.1$, agwStyle=FS.FS_RIGHT)

self.fine_hold_6.SetFormat("\%f")

self.fine_hold_6.SetDigits(2)

self.fine_hold_6.SetFont(wx.Font( 12, 74, 90, 90, False, "Calibri" ) )

self.fine_hold_6.SetForegroundColour(wx.SystemSettings.GetColour( wx.SYS_COLOUR_BTNTEXT) )

self.fine_hold_6.SetBackgroundColour( wx.SystemSettings.GetColour( wx.SYS_COLOUR_BTNSHADOW ) )

fine_parm_sizer_6.Add( self.fine_hold_6, wx.GBPosition( 7, 1), wx.GBSpan( 1,1 ),

wx.BOTTOM $\mid$ wx.RIGHT|wx.LEFT|wx.ALIGN_CENTER_HORIZONTAL, 5 )

self.maxHold_text $=$ wx.StaticText $($ self.fineDisp_panel_6, wx.ID_ANY, u"600", wx.DefaultPosition, wx.DefaultSize, 0 )

self.maxHold_text.Wrap $(-1)$

self.maxHold_text.SetFont( wx.Font( 12, 74, 90, 90, False, "Calibri" ) )

self.maxHold_text.SetForegroundColour( wx.SystemSettings.GetColour( wx.SYS_COLOUR_SCROLLBAR ) )

fine_parm_sizer_6.Add( self.maxHold_text, wx.GBPosition( 7,2 ), wx.GBSpan ( 1,1),

wx.TOP|wx.BOTTOM|wx.LEFT|wx.ALIGN_RIGHT|wx.ALIGN_CENTER_VERTICAL, 5 )

self.fine_radio_panel_6 $=$ wx.Panel( self.fineDisp_panel_6, wx.ID_ANY, wx.DefaultPosition, wx.DefaultSize, wx.TAB_TRAVERSAL )

self.fine_radio_panel_6.SetBackgroundColour( wx.SystemSettings.GetColour( wx.SYS_COLOUR_HIGHLIGHTTEXT ) )

fine_radio_sizer_6 $=$ wx.GridSizer $(0,3,0,0)$

self.cycle_6 = wx.RadioButton( self.fine_radio_panel_6, wx.ID_ANY, u"Cycle", wx.DefaultPosition, wx.DefaultSize, 0 )

fine_radio_sizer_6.Add( self.cycle_6, 0, wx.ALL|wx.ALIGN_CENTER_HORIZONTAL, 5 )

self.cycle_6.SetFont( wx.Font (12, 74, 90, 90, False, "Calibri" ) )

self.cycle_6.SetValue(True)

self.retract_6 $=$ wx.RadioButton( self.fine_radio_panel_6, wx.ID_ANY, u"Retract", wx.DefaultPosition, wx.DefaultSize, 0 )

fine_radio_sizer_6.Add( self.retract_6, 0, wx.ALL|wx.ALIGN_CENTER_HORIZONTAL, 5 )

self.retract_6.SetFont( wx.Font ( 12, 74, 90, 90, False, "Calibri" ) )

self.extend_6 = wx.RadioButton( self.fine_radio_panel_6, wx.ID_ANY, u"Extend", wx.DefaultPosition, wx.DefaultSize, 0 )

fine_radio_sizer_6.Add( self.extend_6, 0, wx.ALL|wx.ALIGN_CENTER_HORIZONTAL, 5 )

self.extend_6.SetFont( wx.Font( 12, 74, 90, 90, False, "Calibri" ) )

self.fine_radio_panel_6.SetSizer(fine_radio_sizer_6 )

self.fine_radio_panel_6.Layout()

fine_radio_sizer_6.Fit( self.fine_radio_panel_6 )

fine_parm_sizer_6.Add( self.fine_radio_panel_6, wx.GBPosition ( 8, 0 ), wx.GBSpan( 1, 3 ), wx.EXPAND |wx.ALL, 5 )

fineDisp_sizer_6.Add( fine_parm_sizer_6, 1, wx.ALIGN_CENTER_HORIZONTAL|wx.ALIGN_CENTER_VERTICAL, 5 )

fine_button_sizer_6 $=$ wx.BoxSizer( wx.VERTICAL ) 
self.fine_indent_button_6 = wx.Button( self.fineDisp_panel_6, 27, u"Indent", wx.DefaultPosition, wx.Size( 175,60 ), 0 ) self.fine_indent_button_6.SetFont( wx.Font( 18, 74, 90, 92, False, "Calibri" ) )

self.Bind(wx.EVT_BUTTON,self.fine_Disp_6_Command,id=27)

fine_button_sizer_6.Add( self.fine_indent_button_6, 0, wx.ALIGN_CENTER_HORIZONTAL|wx.ALL, 5 )

fineDisp_sizer_6.Add( fine_button_sizer_6, 1, wx.EXPAND, 5 )

self.m_panel78 = wx.Panel( self.fineDisp_panel_6, wx.ID_ANY, wx.DefaultPosition, wx.DefaultSize, wx.SIMPLE_BORDER )

gbSizer16 = wx.GridBagSizer $(0,0)$

gbSizer16.SetFlexibleDirection( wx.BOTH )

gbSizer16.SetNonFlexibleGrowMode( wx.FLEX_GROWMODE_SPECIFIED )

fine_priorText_6 $=$ wx.BoxSizer( wx.VERTICAL )

self.prior_Disp_text_6 = wx.StaticText( self.m_panel78, wx.ID_ANY, u"Prior displacements", wx.DefaultPosition, wx.DefaultSize, 0 )

self.prior_Disp_text_6.Wrap( -1 )

self.prior_Disp_text_6.SetFont( wx.Font( 14, 74, 90, 92, False, "Calibri" ) )

fine_priorText_6.Add( self.prior_Disp_text_6, 0, wx.RIGHT|wx.LEFT, 5 )

gbSizer16.Add( fine_priorText_6, wx.GBPosition( 0,0$)$, wx.GBSpan $(1,1)$, wx.EXPAND, 5 )

self.prior_disp_table_panel6 $=$ wx.ScrolledWindow( self.m_pane178, wx.ID_ANY, wx.DefaultPosition, wx.DefaultSize ,

wx.HSCROLL $\mid$ wx.SIMPLE_BORDER|wx.VSCROLL )

self.prior_disp_table_panel6.SetScrollRate $(5,5)$

self.prior_disp_table_panel6.SetMinSize( wx.Size( 320,350 ))

prior_disp $=$ wx.FlexGridSizer $(0,3,0,0)$

prior_disp.SetFlexibleDirection(wx.BOTH )

prior_disp.SetNonFlexibleGrowMode( wx.FLEX_GROWMODE_SPECIFIED )

self.Disp_panel_6 $=$ wx.Panel( self.prior_disp_table_panel6, wx.ID_ANY, wx.DefaultPosition, wx.DefaultSize, wx.TAB_TRAVERSAL )

self.Disp_panel_6.SetBackgroundColour( wx.SystemSettings.GetColour(wx.SYS_COLOUR_SCROLLBAR ) )

self.Disp_panel_6.SetMinSize( wx.Size $(-1,350)$ )

self.disp_sizer1 $1=$ wx.BoxSizer $($ wx.VERTICAL $)$

self.disp_sizer11.SetMinSize( wx.Size ( $-1,350))$

self.ID_header11 = wx.StaticText( self.Disp_panel_6, wx.ID_ANY, u"displacement $(\mathrm{mm}) "$ ", wx.DefaultPosition, wx.Size ( 100,30 ),

wX.ALIGN_CENTRE)

self.ID_header11.Wrap( -1)

self.ID_header11.SetBackgroundColour( wx.SystemSettings.GetColour(wx.SYS_COLOUR_SCROLLBAR ) )

self.disp_sizer11.Add( self.ID_header11, 0, wx.ALL, 5)

self.type_staticline $11=$ wx.StaticLine( self.Disp_panel_6, wx.ID_ANY, wx.DefaultPosition, wx.DefaultSize, wx.LI_HORIZONTAL )

self.disp_sizer11.Add( self.type_staticline11,0, wx.EXPAND|wx.TOP|wx.BOTTOM, 5 )

self.Disp_panel_6.SetSizer( self.disp_sizer11 )

self.Disp_panel_6.Layout()

self.disp_sizer11.Fit( self.Disp_panel_6 )

prior_disp.Add( self.Disp_panel_6, 1, wx.EXPAND|wx.BOTTOM, 5 )

self.velcity_panel_6 = wx.Panel( self.prior_disp_table_panel6, wx.ID_ANY, wx.DefaultPosition, wx.DefaultSize, wx.TAB_TRAVERSAL )

self.velcity_panel_6.SetBackgroundColour(wx.SystemSettings.GetColour(wx.SYS_COLOUR_3DLIGHT ) )

self.velcity_panel_6.SetMinSize(wx.Size (100,350 ) )

self.velocity_sizer11 $=$ wx.BoxSizer( wx.VERTICAL )

self.velocity_sizer11.SetMinSize (wx.Size $(100,350))$

self.force_header11 = wx.StaticText( self.velcity_panel_6, wx.ID_ANY, u"velocityln(mm/s)", wx.DefaultPosition, wx.Size( $-1,30$ ),

wx.ALIGN_CENTRE )

self.force_header11.Wrap( -1 )

self.velocity_sizer11.Add( self.force_header11, 0, wx.ALL|wx.ALIGN_CENTER_HORIZONTAL, 5 )

self.time_staticline $11=$ wx.StaticLine $($ self.velcity_panel_6, wx.ID_ANY, wx.DefaultPosition, wx.DefaultSize, wx.LI_HORIZONTAL )

self.velocity_sizer11.Add( self.time_staticline11,0, wx.EXPAND|wx.TOP|wx.BOTTOM, 5 )

self.velcity_panel_6.SetSizer( self.velocity_sizer11)

self.velcity_panel_6.Layout()

self.velocity_sizer11.Fit( self.velcity_panel_6 )

prior_disp.Add( self.velcity_panel_6, 1, wx.EXPAND|wx.BOTTOM, 5 )

self.acceleration_panel_6 = wx.Panel( self.prior_disp_table_panel6, wx.ID_ANY, wx.DefaultPosition, wx.DefaultSize, wx.TAB_TRAVERSAL )

self.acceleration_panel_6.SetBackgroundColour(wx.SystemSettings.GetColour(wx.SYS_COLOUR_SCROLLBAR ) )

self.acceleration_panel_6.SetMinSize(wx.Size $(-1,350))$

self.acc_sizer11 = wx.BoxSizer( wx.VERTICAL )

self.acc_sizer11.SetMinSize( wx.Size( 100,350))

self.disp_header11 = wx.StaticText( self.acceleration_panel_6, wx.ID_ANY, u"acceleration $\backslash n(\mathrm{~mm} / \mathrm{s} / \mathrm{s}) "$ ", wx.DefaultPosition, wx.Size( $-1,30$ ), wx.ALIGN_CENTRE )

self.disp_header11.Wrap ( -1 )

self.acc_sizer11.Add( self.disp_header11, 0, wx.ALL|wx.ALIGN_CENTER_HORIZONTAL, 5 )

self.disp_staticline1 $1=$ wx.StaticLine( self.acceleration_panel_6, wx.ID_ANY, wx.DefaultPosition, wx.DefaultSize, wx.LI_HORIZONTAL )

self.acc_sizer11.Add( self.disp_staticline11, 0, wx.EXPAND|wx.TOP|wx.BOTTOM, 5 )

self.acceleration_panel_6.SetSizer(self.acc_sizer11 )

self.acceleration_panel_6.Layout()

self.acc_sizer11.Fit( self.acceleration_panel_6 ) 
prior_disp.Add( self.acceleration_panel_6, 1, wx.EXPAND|wx.BOTTOM, 5 )

self.prior_disp_table_panel6.SetSizer( prior_disp )

self.prior_disp_table_panel6.Layout()

prior_disp.Fit( self.prior_disp_table_panel6 )

gbSizer16.Add( self.prior_disp_table_panel6, wx.GBPosition( 1,1 ), wx.GBSpan( 1,1 ), wx.EXPAND, 5 )

self.m_panel78.SetSizer( gbSizer16 )

self.m_panel78.Layout()

gbSizer16.Fit( self.m_panel78 )

fineDisp_sizer_6.Add( self.m_panel78, 1, wx.EXPAND, 5 )

self.fineDisp_panel_6.SetSizer(fineDisp_sizer_6 )

self.fineDisp_panel_6.Layout()

fineDisp_sizer_step6.Add( self.fineDisp_panel_6, 1, wx.EXPAND, 5 )

self.fineDisp_step6.SetSizer( fineDisp_sizer_step6 )

self.fineDisp_step6.Layout()

fineDisp_sizer_step6.Fit( self.fineDisp_step6 )

self.step6_force_panel.AddPage( self.fineDisp_step6, u"Fine Displacement", False )

self.yoshiExp = wx.Panel(self.step6_force_panel,wx.ID_ANY,wx.DefaultPosition,wx.DefaultSize,wx.TAB_TRAVERSAL)

yoshi_sizer $=$ wx.FlexGridSizer $(0,1,0,0)$

yoshi_sizer.SetFlexibleDirection(wx.BOTH)

yoshi_sizer.SetNonFlexibleGrowMode(wx.FLEX_GROWMODE_SPECIFIED)

self.yoshiExp.SetSizer(yoshi_sizer)

self.yoshiExp.Layout()

yoshi_sizer.Fit( self.yoshiExp )

self.step6_force_panel.AddPage( self.yoshiExp, u"Yoshi Exp Beta", False )

self.indentation_plot_6 = wx.Panel( self.step6_force_panel, wx.ID_ANY, wx.DefaultPosition, wx.DefaultSize, wx.TAB_TRAVERSAL )

indentation_plot_sizer_6 $=$ wx.FlexGridSizer $(0,1,0,0)$

indentation_plot_sizer_6.SetFlexibleDirection( wx.BOTH )

indentation_plot_sizer_6.SetNonFlexibleGrowMode( wx.FLEX_GROWMODE_SPECIFIED )

self.ip_select_text $=$ wx.StaticText( self.indentation_plot_6, wx.ID_ANY, u"Select plots to view", wx.DefaultPosition, wx.Size( 660,-1 ), 0 )

self.ip_select_text.Wrap( -1$)$

self.ip_select_text.SetFont( wx.Font( 14, 74, 90, 92, False, "Calibri" ) )

indentation_plot_sizer_6.Add( self.ip_select_text, 0, wx.TOP|wx.BOTTOM|wx.RIGHT, 5 )

self.ip_force_disp_plot $=$ wx.StaticText( self.indentation_plot_6, wx.ID_ANY, u"Force and displacement plots", wx.DefaultPosition,

wx.DefaultSize, 0 )

self.ip_force_disp_plot.Wrap $(-1)$

self.ip_force_disp_plot.SetFont( wx.Font( 14, 74, 90, 92, False, "Calibri" ) )

indentation_plot_sizer_6.Add( self.ip_force_disp_plot, 0 , wx.RIGHT, 5 )

self.window_6_f_d = wx.ScrolledWindow( self.indentation_plot_6, wx.ID_ANY, wx.DefaultPosition, wx.DefaultSize,

wx.HSCROLL $\mid$ wx.SIMPLE_BORDER|wx.VSCROLL )

self.window_6_f_d.SetScrollRate( 5, 5 )

self.window_6_f_d.SetMinSize( wx.Size( 430,200 ))

self.sizer_6_f_d = wx.FlexGridSizer( 0, 4, 0,0)

self.sizer_6_f_d.SetFlexibleDirection(wx.BOTH )

self.sizer_6_f_d.SetNonFlexibleGrowMode( wx.FLEX_GROWMODE_SPECIFIED )

self.type_panel_f_d_6 $=$ wx.Panel( self.window_6_f_d, wx.ID_ANY, wx.DefaultPosition, wx.DefaultSize, wx.TAB_TRAVERSAL )

self.type_panel_f_d_6.SetBackgroundColour(wx.SystemSettings.GetColour(wx.SYS_COLOUR_SCROLLBAR ) )

self.type_panel_f_d_6.SetMinSize( wx.Size ( $-1,200)$ )

self.type_sizer_f_d_6=wx.BoxSizer(wx.VERTICAL )

self.type_header_f_d_6 = wx.StaticText( self.type_panel_f_d_6, wx.ID_ANY, u"View In Force | Disp.", wx.DefaultPosition, wx.Size( 100,30 ),

wx.ALIGN_CENTRE )

self.type_header_f_d_6.Wrap $(-1)$

self.type_header_f_d_6.SetBackgroundColour(wx.SystemSettings.GetColour(wx.SYS_COLOUR_SCROLLBAR ) )

self.type_sizer_f_d_6.Add( self.type_header_f_d_6, 0, wx.TOP|wx.RIGHT|wx.LEFT, 5 )

self.type_line_f_d_6 = wx.StaticLine( self.type_panel_f_d_6, wx.ID_ANY, wx.DefaultPosition, wx.DefaultSize, wx.LI_HORIZONTAL )

self.type_sizer_f_d_6.Add( self.type_line_f_d_6, 0, wx.EXPAND|wx.TOP|wx.BOTTOM, 5 )

self.type_gridSizer_f_d_6 = wx.GridSizer $(0,2,0,0)$

noText $=$ wx.StaticText $($ self.type_panel_f_d_6, wx.ID_ANY, u" ", wx.DefaultPosition, wx.DefaultSize, wx.ALIGN_LEFT )

self.type_gridSizer_f_d_6.Add(noText,0,wx.ALL|wx.ALIGN_CENTER_HORIZONTAL, 5)

self.type_sizer_f_d_6.Add( self.type_gridSizer_f_d_6, 1, wx.EXPAND, 5 )

self.type_panel_f_d_6.SetSizer( self.type_sizer_f_d_6)

self.type_panel_f_d_6.Layout()

self.type_sizer_f_d_6.Fit( self.type_panel_f_d_6 )

self.sizer_6_f_d.Add( self.type_panel_f_d_6, 1, wx.ALIGN_CENTER_HORIZONTAL|wx.EXPAND, 5 )

self.force_panel_f_d_6 = wx.Panel( self.window_6_f_d, wx.ID_ANY, wx.DefaultPosition, wx.DefaultSize, wx.TAB_TRAVERSAL )

self.force_panel_f_d_6.SetBackgroundColour( wx.SystemSettings.GetColour( wx.SYS_COLOUR_3DLIGHT ) )

self.force_panel_f_d_6.SetMinSize(wx.Size (100,200))

self.force_sizer_f_d_6 $=$ wx.BoxSizer(wx.VERTICAL )

self.force_sizer_f_d_6.SetMinSize( wx.Size $(100,-1)$ ) 
self.force_header_f_d_6 $=$ wx.StaticText( self.force_panel_f_d_6, wx.ID_ANY, u"Forceln(mN)", wx.DefaultPosition, wx.Size( $-1,30$ ), wx.ALIGN_CENTRE )

self.force_header_f_d_6.Wrap( -1 )

self.force_sizer_f_d_6.Add( self.force_header_f_d_6, 0, wx.ALIGN_CENTER_HORIZONTAL|wx.TOP|wx.RIGHT|wx.LEFT, 5 )

self.time_line_f_d_6 = wx.StaticLine( self.force_panel_f_d_6, wx.ID_ANY, wx.DefaultPosition, wx.DefaultSize, wx.LI_HORIZONTAL ) self.force_sizer_f_d_6.Add( self.time_line_f_d_6, 0, wx.EXPAND|wx.TOP|wx.BOTTOM, 5 )

self.force_panel_f_d_6.SetSizer( self.force_sizer_f_d_6 )

self.force_panel_f_d_6.Layout()

self.force_sizer_f_d_6.Fit( self.force_panel_f_d_6 )

self.sizer_6_f_d.Add( self.force_panel_f_d_6, 1, wx.EXPAND, 5 )

self.disp_panel_f_d_6 = wx.Panel( self.window_6_f_d, wx.ID_ANY, wx.DefaultPosition, wx.DefaultSize, wx.TAB_TRAVERSAL )

self.disp_panel_f_d_6.SetBackgroundColour(wx.SystemSettings.GetColour( wx.SYS_COLOUR_SCROLLBAR ) )

self.disp_panel_f_d_6.SetMinSize (wx.Size $(-1,200))$

self.disp_sizer_f_d_6 $=$ wx.BoxSizer( wx.VERTICAL $)$

self.disp_sizer_f_d_6.SetMinSize( wx.Size (100,-1 ))

self.disp_header_f_d_6 = wx.StaticText( self.disp_panel_f_d_6, wx.ID_ANY, u"Displacement $\ln (\mathrm{mm})$ ", wx.DefaultPosition, wx.Size ( $-1,30$ ), wx.ALIGN_CENTRE )

self.disp_header_f_d_6.Wrap( -1 )

self.disp_sizer_f_d_6.Add( self.disp_header_f_d_6, 0, wx.ALIGN_CENTER_HORIZONTAL|wx.TOP|wx.RIGHT|wx.LEFT, 5 )

self.disp_line_f_d_6 = wx.StaticLine( self.disp_panel_f_d_6, wx.ID_ANY, wx.DefaultPosition, wx.DefaultSize, wx.LI_HORIZONTAL )

self.disp_sizer_f_d__6.Add( self.disp_line_f_d_6, 0, wx.EXPAND|wx.TOP|wx.BOTTOM, 5 )

self.disp_panel_f_d_6.SetSizer( self.disp_sizer_f_d_6 )

self.disp_panel_f_d_6.Layout()

self.disp_sizer_f_d_6.Fit( self.disp_panel_f_d_6 )

self.sizer_6_f_d.Add( self.disp_panel_f_d_- 6,1 , wx.EXPAND, 5 )

self.velocity_panel_f_d_6 = wx.Panel( self.window_6_f_d, wx.ID_ANY, wx.DefaultPosition, wx.DefaultSize, wx.TAB_TRAVERSAL )

self.velocity_panel_f_d_6.SetBackgroundColour( wx.SystemSettings.GetColour( wx.SYS_COLOUR_3DLIGHT ) )

self.velocity_panel_f_d_6.SetMinSize(wx.Size $(-1,200)$ )

self.velocity_sizer_f_d_6 = wx.BoxSizer(wx.VERTICAL )

self.velocity_sizer_f_d_6.SetMinSize( wx.Size $(-1,200)$ )

self.velocity_header_f_d_6 = wx.StaticText( self.velocity_panel_f_d_6, wx.ID_ANY, u"Velocity $\backslash n(\mathrm{~mm} / \mathrm{s})$ ", wx.DefaultPosition, wx.Sizer

100,30), wx.ALIGN_CENTRE )

self.velocity_header_f_d_6.Wrap( -1 )

self.velocity_header_f_d_6.SetBackgroundColour(wx.SystemSettings.GetColour(wx.SYS_COLOUR_3DLIGHT ) )

self.velocity_sizer_f_d_6.Add( self.velocity_header_f_d_6, 0, wx.TOP|wx.RIGHT|wx.LEFT, 5 )

self.velocity_line_f_d_6 = wx.StaticLine( self.velocity_panel_f_d_6, wx.ID_ANY, wx.DefaultPosition, wx.DefaultSize, wx.LI_HORIZONTAL )

self.velocity_sizer_f_d_6.Add( self.velocity_line_f_d_6, 0, wx.EXPAND|wx.TOP|wx.BOTTOM, 5 )

self.velocity_panel_f_d_t6.SetSizer( self.velocity_sizer_f_d_6 )

self.velocity_panel_f_d_6.Layout()

self.velocity_sizer_f_d_6.Fit( self.velocity_panel_f_d_6 )

self.sizer_6_f_d.Add( self.velocity_panel_f_d_6, 1, wx.EXPAND, 5 )

self.window_6_f_d.SetSizer( self.sizer_6_f_d )

self.window_6_f_d.Layout()

self.sizer_6_f_d.Fit( self.window_6_f_d )

indentation_plot_sizer_6.Add( self.window_6_f_d, 0,

wx.ALIGN_CENTER_HORIZONTAL|wx.ALIGN_CENTER_VERTICAL|wx.BOTTOM|wx.RIGHT|wx.LEFT, 5 )

self.fine_disp_text_6 = wx.StaticText( self.indentation_plot_6, wx.ID_ANY, u"Fine displacement plots", wx.DefaultPosition, wx.DefaultSize, 0 ) self.fine_disp_text_6.Wrap( -1 )

self.fine_disp_text_6.SetFont( wx.Font( 14, 74, 90, 92, False, "Calibri" ) )

indentation_plot_sizer_6.Add( self.fine_disp_text_6, 0, wx.RIGHT, 5 )

self.window_6_fd = wx.ScrolledWindow( self.indentation_plot_6, wx.ID_ANY, wx.DefaultPosition, wx.DefaultSize,

wx.HSCROLL $\mid$ wx.SIMPLE_BORDER/wx.VSCROLL )

self.window_6_fd.SetScrollRate( 5, 5)

self.window_6_fd.SetMinSize( wx.Size( 430,200 ) )

sizer_6_fd $=$ wx.FlexGridSizer $(0,7,0,0)$

sizer_6_fd.SetFlexibleDirection(wx.BOTH )

sizer_6_fd.SetNonFlexibleGrowMode( wx.FLEX_GROWMODE_SPECIFIED)

self.type_panel_fd_6 = wx.Panel( self.window_6_fd, wx.ID_ANY, wx.DefaultPosition, wx.DefaultSize, wx.TAB_TRAVERSAL )

self.type_panel_fd_6.SetBackgroundColour( wx.SystemSettings.GetColour( wx.SYS_COLOUR_SCROLLBAR ) )

self.type_panel_fd_6.SetMinSize(wx.Size $(-1,200))$

self.type_sizer_fd_6 = wx.BoxSizer(wx.VERTICAL)

self.type_sizer_fd_6.SetMinSize( wx.Size $(-1,200))$

self.type_header_fd_6 =wx.StaticText( self.type_panel_fd_6, wx.ID_ANY, u"View ", wx.DefaultPosition, wx.Size( 100,30 ),

wX.ALIGN_CENTRE )

self.type_header_fd_6.Wrap( -1 )

self.type_header_fd_6.SetBackgroundColour(wx.SystemSettings.GetColour(wx.SYS_COLOUR_SCROLLBAR ) )

self.type_sizer_fd_6.Add( self.type_header_fd_6, 0, wx.TOP|wx.RIGHT|wx.LEFT, 5 )

self.type_line_fd_6 = wx.StaticLine( self.type_panel_fd_6, wx.ID_ANY, wx.DefaultPosition, wx.DefaultSize, wx.LI_HORIZONTAL )

self.type_sizer_fd_6.Add( self.type_line_fd_6, 0, wx.EXPAND|wx.TOP|wx.BOTTOM, 5 ) 
self.type_panel_fd_6.SetSizer( self.type_sizer_fd_6 )

self.type_panel_fd_6.Layout()

self.type_sizer_fd_6.Fit( self.type_panel_fd_6)

sizer_6_fd.Add( self.type_panel_fd_6, 1, wx.ALIGN_CENTER_HORIZONTAL|wx.EXPAND, 5 )

self.disp_panel_fd_6 = wx.Panel( self.window_6_fd, wx.ID_ANY, wx.DefaultPosition, wx.DefaultSize, wx.TAB_TRAVERSAL )

self.disp_panel_fd_6.SetBackgroundColour(wx.SystemSettings.GetColour( wx.SYS_COLOUR_3DLIGHT ) )

self.disp_panel_fd_6.SetMinSize( wx.Size (100,200))

self.disp_sizer_fd_6 = wx.BoxSizer( wx.VERTICAL )

self.disp_sizer_fd_6.SetMinSize( wx.Size( 100,-1 ))

self.disp_header_fd_6 $=$ wx.StaticText( self.disp_panel_fd_6, wx.ID_ANY, u"Displacement $\ln (\mathrm{mm})$ ", wx.DefaultPosition, wx.Size( $-1,30$ ),

wx.ALIGN_CENTRE )

self.disp_header_fd_6.Wrap( -1 )

self.disp_sizer_fd_6.Add( self.disp_header_fd_6, 0, wx.ALIGN_CENTER_HORIZONTAL|wx.TOP|wx.RIGHT|wx.LEFT, 5 )

self.disp_line_fd_6 $=$ wx.StaticLine( self.disp_panel_fd_6, wx.ID_ANY, wx.DefaultPosition, wx.DefaultSize, wx.LI_HORIZONTAL )

self.disp_sizer_fd_6.Add( self.disp_line_fd_6, 0, wx.EXPAND|wx.TOP|wx.BOTTOM, 5 )

self.disp_panel_fd_6.SetSizer( self.disp_sizer_fd_6 )

self.disp_panel_fd_6.Layout()

self.disp_sizer_fd_6.Fit( self.disp_panel_fd_6)

sizer_6_fd.Add( self.disp_panel_fd_6, 1, wx.EXPAND, 5 )

self.vel_panel_fd_6 = wx.Panel( self.window_6_fd, wx.ID_ANY, wx.DefaultPosition, wx.DefaultSize, wx.TAB_TRAVERSAL )

self.vel_panel_fd_6.SetBackgroundColour( wx.SystemSettings.GetColour( wx.SYS_COLOUR_SCROLLBAR $)$ )

self.vel_panel_fd_6.SetMinSize (wx.Size $(-1,350)$ )

self.vel_sizer_fd_6 $=$ wx.BoxSizer(wx.VERTICAL )

self.vel_sizer_fd_6.SetMinSize( wx.Size( $100,-1)$ )

self.vel_header_fd_6 = wx.StaticText( self.vel_panel_fd_6, wx.ID_ANY, u"Velocityln(mm/s)", wx.DefaultPosition, wx.Size( $-1,30$ ),

wx.ALIGN_CENTRE )

self.vel_header_fd_6.Wrap( -1 )

self.vel_sizer_fd_6.Add( self.vel_header_fd_6, 0, wx.ALIGN_CENTER_HORIZONTAL|wx.TOP|wx.RIGHT|wx.LEFT, 5 )

self.vel_line_fd_6 = wx.StaticLine( self.vel_panel_fd_6, wx.ID_ANY, wx.DefaultPosition, wx.DefaultSize, wx.LI_HORIZONTAL )

self.vel_sizer_fd_6.Add( self.vel_line_fd_6, 0, wx.EXPAND|wx.TOP|wx.BOTTOM, 5 )

self.vel_panel_fd_6.SetSizer( self.vel_sizer_fd_6)

self.vel_panel_fd_6.Layout()

self.vel_sizer_fd_6.Fit( self.vel_panel_fd_6 )

sizer_6_fd.Add( self.vel_panel_fd_6, 1, wx.EXPAND, 5 )

self.acc_panel_fd_6 = wx.Panel( self.window_6_fd, wx.ID_ANY, wx.DefaultPosition, wx.DefaultSize, wx.TAB_TRAVERSAL )

self.acc_panel_fd_6.SetBackgroundColour( wx.SystemSettings.GetColour(wx.SYS_COLOUR_3DLIGHT ) )

self.acc_panel_fd_6.SetMinSize( wx.Size $(-1,350))$

self.acc_sizer_fd_6 =wx.BoxSizer(wx.VERTICAL )

self.acc_header_fd_6 = wx.StaticText( self.acc_panel_fd_6, wx.ID_ANY, u"Acceleration $\backslash n(\mathrm{~mm} / \mathrm{s} / \mathrm{s})$ ", wx.DefaultPosition, wx.Size( 100,30 ), wx.ALIGN_CENTRE )

self.acc_header_fd_6.Wrap $(-1)$

self.acc_header_fd_6.SetBackgroundColour(wx.SystemSettings.GetColour(wx.SYS_COLOUR_3DLIGHT ))

self.acc_sizer_fd_6.Add( self.acc_header_fd_6, 0, wx.TOP|wx.RIGHT|wx.LEFT, 5 )

self.acc_line_fd_6 $=$ wx.StaticLine( self.acc_panel_fd_6, wx.ID_ANY, wx.DefaultPosition, wx.DefaultSize, wx.LI_HORIZONTAL )

self.acc_sizer_fd_6.Add( self.acc_line_fd_6, 0, wx.EXPAND|wx.TOP|wx.BOTTOM, 5 )

self.acc_panel_fd_6.SetSizer( self.acc_sizer_fd_6 )

self.acc_panel_fd_6.Layout()

self.acc_sizer_fd_6.Fit( self.acc_panel_fd_6 )

sizer_6_fd.Add( self.acc_panel_fd_6, 1 , wx.EXPAND, 5 )

self.window_6_fd.SetSizer( sizer_6_fd )

self.window_6_fd.Layout()

sizer_6_fd.Fit( self.window_6_fd )

indentation_plot_sizer_6.Add( self.window_6_fd, 0, wx.BOTTOM|wx.RIGHT|wx.LEFT|wx.ALIGN_CENTER_HORIZONTAL, 5 )

self.viewButton_ip_6 = wx.Button( self.indentation_plot_6, 28, u"View", wx.DefaultPosition, wx.Size( 175,60 ), 0 )

self.viewButton_ip_6.SetFont( wx.Font( 18, 74, 90, 92, False, "Calibri" ) )

self.Bind(wx.EVT_BUTTON,self.view_ip,id=28)

indentation_plot_sizer_6.Add( self.viewButton_ip_6, 0, wx.ALL|wx.ALIGN_CENTER_HORIZONTAL, 5 )

self.indentation_plot_6.SetSizer( indentation_plot_sizer_6 )

self.indentation_plot_6.Layout()

indentation_plot_sizer_6.Fit( self.indentation_plot_6 )

self.step6_force_panel.AddPage( self.indentation_plot_6, u"Indentation Plots", False )

decisionSizer.Add( self.step6_force_panel, 1, wx.EXPAND, 5 )

self.step5_disp_panel $=$ wx.Notebook $($ self.decisionPanel, wx.ID_ANY, wx.DefaultPosition, wx.DefaultSize, wx.NB_TOP)

self.step5_disp_panel.Enable( False )

self.step5_disp_panel.Hide()

self.fine_disp_panel_5 = wx.Panel( self.step5_disp_panel, wx.ID_ANY, wx.DefaultPosition, wx.DefaultSize, wx.TAB_TRAVERSAL )

fine_disp_sizer_5 = wx.FlexGridSizer $(0,1,0,0)$ 
fine_disp_sizer_5.SetFlexibleDirection(wx.BOTH )

fine_disp_sizer_5.SetNonFlexibleGrowMode( wx.FLEX_GROWMODE_SPECIFIED )

self.indentParm_text_5 = wx.StaticText( self.fine_disp_panel_5, wx.ID_ANY, u"Indentation parameters", wx.DefaultPosition, wx.Size( 660,-1 ), 0 )

self.indentParm_text_5.Wrap( -1 )

self.indentParm_text_5.SetFont( wx.Font( 14, 74, 90, 92, False, "Calibri" ) )

fine_disp_sizer_5.Add( self.indentParm_text_5, 0, wx.TOP|wx.BOTTOM|wx.LEFT, 5 )

param_dp_5 = wx.GridBagSizer $(0,0)$

param_dp_5.SetFlexibleDirection( wx.BOTH )

param_dp_5.SetNonFlexibleGrowMode( wx.FLEX_GROWMODE_SPECIFIED)

self.max_text_dp_5 = wx.StaticText( self.fine_disp_panel_5, wx.ID_ANY, u"Max", wx.DefaultPosition, wx.DefaultSize, 0 )

self.max_text_dp_5.Wrap( -1$)$

self.max_text_dp_5.SetFont( wx.Font( 12, 74, 90, 90, False, "Calibri" ) )

self.max_text_dp_5.SetForegroundColour(wx.SystemSettings.GetColour( wx.SYS_COLOUR_SCROLLBAR ) )

param_dp_5.Add( self.max_text_dp_5,wx.GBPosition( 0,2$)$, wx.GBSpan $(1,1)$,

wx.ALIGN_RIGHT|wx.ALIGN_CENTER_VERTICAL/wx.LEFT|wx.BOTTOM|wx.TOP, 5 )

self.displacement_text_dp_5 = wx.StaticText( self.fine_disp_panel_5, wx.ID_ANY, u"displ. (mm)", wx.DefaultPosition, wx.DefaultSize,

wx.ALIGN_CENTRE )

self.displacement_text_dp_5.Wrap (-1)

self.displacement_text_dp_5.SetFont( wx.Font( 12, 74, 90, 90, False, "Calibri" ) )

param_dp_5.Add( self.displacement_text_dp_5, wx.GBPosition( 0,1 ), wx.GBSpan ( 1,1 ),

wx.LEFT|wx.RIGHT|wx.ALIGN_LEFT|wx.ALIGN_BOTTOM, 5 )

self.min_text_dp_5 = wx.StaticText( self.fine_disp_panel_5, wx.ID_ANY, u"Min", wx.DefaultPosition, wx.DefaultSize, wx.ALIGN_CENTRE ) self.min_text_dp_5.Wrap( -1$)$

self.min_text_dp_5.SetFont( wx.Font( 12, 74, 90, 90, False, "Calibri" ) )

self.min_text_dp_5.SetForegroundColour(wx.SystemSettings.GetColour( wx.SYS_COLOUR_SCROLLBAR ) )

param_dp_5.Add( self.min_text_dp_5, wx.GBPosition( 0,0), wx.GBSpan $(1,1)$,

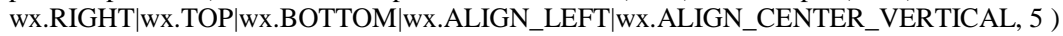

self.minDisp_text_dp_5 = wx.StaticText( self.fine_disp_panel_5, wx.ID_ANY, u"0.01", wx.DefaultPosition, wx.Size( -1,-1 ), wx.ALIGN_LEFT

self.minDisp_text_dp_5.Wrap( -1$)$

self.minDisp_text_dp_5.SetFont( wx.Font( 12, 74, 90, 90, False, "Calibri" ) )

self.minDisp_text_dp_5.SetForegroundColour(wx.SystemSettings.GetColour( wx.SYS_COLOUR_SCROLLBAR ) )

self.minDisp_text_dp_5.SetMinSize( wx.Size( 100,-1 ) )

param_dp_5.Add( self.minDisp_text_dp_5, wx.GBPosition( 1,0 ), wx.GBSpan( 1,1 ),

wx.ALIGN_LEFT|wx.TOP|wx.BOTTOM|wx.ALIGN_CENTER_VERTICAL, 5 )

self.disp_spin_dp_5 = FS.FloatSpin( self.fine_disp_panel_5,-1, value $=1$, min_val=.01, max_val $=5$,increment $=.01$,agwStyle=FS.FS_RIGHT )

self.disp_spin_dp_5.SetFormat("\%f")

self.disp_spin_dp_5.SetDigits(2)

self.disp_spin_dp_5.SetFont( wx.Font( 12, 74, 90, 90, False, "Calibri" ) )

self.disp_spin_dp_5.SetForegroundColour( wx.SystemSettings.GetColour(wX.SYS_COLOUR_BTNTEXT ) )

self.disp_spin_dp_5.SetBackgroundColour( wx.SystemSettings.GetColour( wx.SYS_COLOUR_BTNSHADOW ) )

param_dp_5.Add( self.disp_spin_dp_5, wx.GBPosition( 1,1 ), wx.GBSpan $(1,1)$,

wx.BOTTOM|wx.RIGHT|wx.LEFT|wx.ALIGN_CENTER_HORIZONTAL, 5 )

self.minVel_text_dp_5 = wx.StaticText( self.fine_disp_panel_5, wx.ID_ANY, u"1.0", wx.DefaultPosition, wx.Size( $-1,-1$ ),

wX.ALIGN_CENTRE )

self.minVel_text_dp_5.Wrap( -1 )

self.minVel_text_dp_5.SetFont( wx.Font( 12, 74, 90, 90, False, "Calibri" ) )

self.minVel_text_dp_5.SetForegroundColour(wx.SystemSettings.GetColour(wx.SYS_COLOUR_SCROLLBAR ) )

param_dp_5.Add( self.minVel_text_dp_5, wx.GBPosition( 3,0), wx.GBSpan $(1,1)$,

wx.ALIGN_LEFT|wx.TOP|wx.BOTTOM|wx.RIGHT|wx.ALIGN_CENTER_VERTICAL, 5 )

self.max_dispText_dp_5 = wx.StaticText( self.fine_disp_panel_5, wx.ID_ANY, u"5", wx.DefaultPosition, wx.DefaultSize, wx.ALIGN_RIGHT )

self.max_dispText_dp_5.Wrap( -1 )

self.max_dispText_dp_5.SetFont( wx.Font (12, 74, 90, 90, False, "Calibri" ) )

self.max_dispText_dp_5.SetForegroundColour(wx.SystemSettings.GetColour(wx.SYS_COLOUR_SCROLLBAR ) )

self.max_dispText_dp_5.SetMinSize( wx.Size( 100,-1 ) )

param_dp_5.Add( self.max_dispText_dp_5, wx.GBPosition( 1,2), wx.GBSpan( 1,1 ),

wx.TOP|wx.BOTTOM|wx.ALIGN_RIGHT|wx.LEFT|wx.ALIGN_CENTER_VERTICAL, 5 )

self.vel_text_dp_5 = wx.StaticText( self.fine_disp_panel_5, wx.ID_ANY, u"velocity (mm/s)", wx.DefaultPosition, wx.DefaultSize,

wx.ALIGN_CENTRE )

self.vel_text_dp_5.Wrap( -1 )

self.vel_text_dp_5.SetFont( wx.Font( 12, 74, 90, 90, False, "Calibri" ) )

param_dp_5.Add( self.vel_text_dp_5, wx.GBPosition( 2, 1 ), wx.GBSpan( 1,1 ), wx.ALIGN_LEFT|wx.LEFT|wx.RIGHT|wx.TOP, 5 )

self.vel_spin_dp_5 $=$ FS.FloatSpin( self.fine_disp_panel_5, $-1, \min \_v a l=1, \max \_v a l=40.0$, increment $=.01$,agwStyle=FS.FS_RIGHT )

self.vel_spin_dp_5.SetFormat("\%f")

self.vel_spin_dp_5.SetDigits(2)

self.vel_spin_dp_5.SetFont( wx.Font( 12, 74, 90, 90, False, "Calibri" ) ) 
self.vel_spin_dp_5.SetForegroundColour(wx.SystemSettings.GetColour( wx.SYS_COLOUR_BTNTEXT ) )

self.vel_spin_dp_5.SetBackgroundColour(wx.SystemSettings.GetColour(wx.SYS_COLOUR_BTNSHADOW ) )

param_dp_5.Add( self.vel_spin_dp_5, wx.GBPosition( 3,1),wx.GBSpan( 1,1 ),

wx.BOTTOM|wx.RIGHT|wx.LEFT|wx.ALIGN_CENTER_HORIZONTAL, 5 )

self.maxVel_text_dp_5 = wx.StaticText( self.fine_disp_panel_5, wx.ID_ANY, u"40", wx.DefaultPosition, wx.DefaultSize, 0 )

self.maxVel_text_dp_5.Wrap( -1 )

self.maxVel_text_dp_5.SetFont( wx.Font( 12, 74, 90, 90, False, "Calibri" ) )

self.maxVel_text_dp_5.SetForegroundColour( wx.SystemSettings.GetColour(wx.SYS_COLOUR_SCROLLBAR ) )

param_dp_5.Add( self.maxVel_text_dp_5, wx.GBPosition( 3,2 ), wx.GBSpan (1,1),

wx.TOP|wx.BOTTOM|wx.LEFT|wx.ALIGN_RIGHT|wx.ALIGN_CENTER_VERTICAL, 5 )

self.acc_text_dp_5 = wx.StaticText( self.fine_disp_panel_5, wx.ID_ANY, u"accel. (mm/s/s)", wx.DefaultPosition, wx.DefaultSize, wx.ALIGN_CENTRE )

self.acc_text_dp_5.Wrap $(-1)$

self.acc_text_dp_5.SetFont( wx.Font( 12, 74, 90, 90, False, "Calibri" ) )

param_dp_5.Add( self.acc_text_dp_5, wx.GBPosition ( 4, 1 ), wx.GBSpan( 1,1 ), wx.TOP|wx.RIGHT|wx.LEFT|wx.ALIGN_LEFT, 5 )

self.min_Acc_text_dp_5 = wx.StaticText( self.fine_disp_panel_5, wx.ID_ANY, u"1.0", wx.DefaultPosition, wx.DefaultSize, 0 )

self.min_Acc_text_dp_5.Wrap( -1 )

self.min_Acc_text_dp_5.SetFont(wx.Font( 12, 74, 90, 90, False, "Calibri" ) )

self.min_Acc_text_dp_5.SetForegroundColour(wx.SystemSettings.GetColour(wx.SYS_COLOUR_SCROLLBAR ) )

param_dp_5.Add( self.min_Acc_text_dp_5, wx.GBPosition( 5, 0 ), wx.GBSpan $(1,1)$,

wx.ALIGN_LEFT|wx.TOP|wx.BOTTOM|wx.RIGHT|wx.ALIGN_CENTER_VERTICAL, 5 )

self.acc_spin_dp_5 = FS.FloatSpin( self.fine_disp_panel_5, $-1, \min \_v a l=1, \max \_v a l=160.0$,increment $=1$, agwStyle=FS.FS_RIGHT )

self.acc_spin_dp_5.SetFormat("\%f")

self.acc_spin_dp_5.SetDigits(2)

self.acc_spin_dp_5.SetFont( wx.Font( 12, 74, 90, 90, False, "Calibri" ) )

self.acc_spin_dp_5.SetForegroundColour(wx.SystemSettings.GetColour(wx.SYS_COLOUR_BTNTEXT) )

self.acc_spin_dp_5.SetBackgroundColour( wx.SystemSettings.GetColour( wx.SYS_COLOUR_BTNSHADOW ) )

param_dp_5.Add( self.acc_spin_dp_5, wx.GBPosition( 5,1),wx.GBSpan $(1,1)$,

wx.BOTTOM|wx.RIGHT|wx.LEFT|wx.ALIGN_CENTER_HORIZONTAL, 5 )

self.max_Acc_text_dp_5 = wx.StaticText( self.fine_disp_panel_5, wx.ID_ANY, u"160", wx.DefaultPosition, wx.DefaultSize, 0 )

self.max_Acc_text_dp_5.Wrap( -1$)$

self.max_Acc_text_dp_5.SetFont( wx.Font( 12, 74, 90, 90, False, "Calibri" ) )

self.max_Acc_text_dp_5.SetForegroundColour(wx.SystemSettings.GetColour( wx.SYS_COLOUR_SCROLLBAR ) )

param_dp_5.Add( self.max_Acc_text_dp_5, wx.GBPosition( 5,2 ), wx.GBSpan( 1,1),

wx.LEFT|wx.TOP|wx.BOTTOM|wx.ALIGN_RIGHT|wx.ALIGN_CENTER_VERTICAL, 5 )

self.hold_text_dp_5 = wx.StaticText( self.fine_disp_panel_5, wx.ID_ANY, u"hold (s) ", wx.DefaultPosition, wx.DefaultSize,

wX.ALIGN_CENTRE)

self.hold_text_dp_5.Wrap (-1)

self.hold_text_dp_5.SetFont( wx.Font( 12, 74, 90, 90, False, "Calibri" ) )

param_dp_5.Add( self.hold_text_dp_5, wx.GBPosition( 6,1$)$, wx.GBSpan( 1,1$)$,

wx.TOP|wx.RIGHT|wx.LEFT|wx.ALIGN_LEFT|wx.ALIGN_CENTER_VERTICAL, 5 )

self.min_holdText_dp_5 = wx.StaticText( self.fine_disp_panel_5, wx.ID_ANY, u"1", wx.DefaultPosition, wx.DefaultSize,

wX.ALIGN_CENTRE )

self.min_holdText_dp_5.Wrap( -1 )

self.min_holdText_dp_5.SetFont( wx.Font( 12, 74, 90, 90, False, "Calibri" ) )

self.min_holdText_dp_5.SetForegroundColour(wx.SystemSettings.GetColour(wx.SYS_COLOUR_SCROLLBAR ) )

param_dp_5.Add( self.min_holdText_dp_5, wx.GBPosition( 7, 0 ), wx.GBSpan ( 1,1

),wx.ALIGN_LEFT|wx.TOP|wx.BOTTOM|wx.RIGHT|wx.ALIGN_CENTER_VERTICAL, 5 )

self.hold_spin_dp_5 = FS.FloatSpin ( self.fine_disp_panel_5, $-1, \min \_v a l=1, \max \_v a l=600$,increment $=.5$, agwStyle=FS.FS_RIGHT )

self.hold_spin_dp_5.SetFormat("\%f")

self.hold_spin_dp_5.SetDigits(2)

self.hold_spin_dp_5.SetFont( wx.Font( 12, 74, 90, 90, False, "Calibri" ) )

self.hold_spin_dp_5.SetForegroundColour( wx.SystemSettings.GetColour(wx.SYS_COLOUR_BTNTEXT ) )

self.hold_spin_dp_5.SetBackgroundColour(wx.SystemSettings.GetColour( wx.SYS_COLOUR_BTNSHADOW ) )

param_dp_5.Add( self.hold_spin_dp_5, wx.GBPosition( 7,1 ), wx.GBSpan( 1,1 ),

wx.BOTTOM|wx.RIGHT|wx.LEFT|wx.ALIGN_CENTER_HORIZONTAL, 5 )

self.maxHold_text_dp_5 = wx.StaticText( self.fine_disp_panel_5, wx.ID_ANY, u"600", wx.DefaultPosition, wx.DefaultSize, 0 )

self.maxHold_text_dp_5.Wrap $(-1)$

self.maxHold_text_dp_5.SetFont( wx.Font( 12, 74, 90, 90, False, "Calibri" ) )

self.maxHold_text_dp_5.SetForegroundColour(wx.SystemSettings.GetColour(wx.SYS_COLOUR_SCROLLBAR ) )

param_dp_5.Add( self.maxHold_text_dp_5, wx.GBPosition( 7,2), wx.GBSpan ( 1,1),

wx.LEFT|wx.TOP|wx.BOTTOM|wx.ALIGN_RIGHT|wx.ALIGN_CENTER_VERTICAL, 5 )

self.CRE_panel_5 = wx.Panel( self.fine_disp_panel_5, wx.ID_ANY, wx.DefaultPosition, wx.DefaultSize, wx.TAB_TRAVERSAL )

self.CRE_panel_5.SetBackgroundColour(wx.SystemSettings.GetColour(wx.SYS_COLOUR_HIGHLIGHTTEXT ) )

CRE_sizer_5 = wx.GridSizer $(0,3,0,0)$

self.cycle_dp_5 $=$ wx.RadioButton( self.CRE_panel_5, wx.ID_ANY, u"Cycle", wx.DefaultPosition, wx.DefaultSize, 0 )

CRE_sizer_5.Add( self.cycle_dp_5, 0, wx.ALL|wx.ALIGN_CENTER_HORIZONTAL, 5 )

self.cycle_dp_5.SetFont( wx.Font( 12, 74, 90, 90, False, "Calibri" ) )

self.cycle_dp_5.SetValue(True)

self.retract_dp_5 = wx.RadioButton( self.CRE_panel_5, wx.ID_ANY, u"Retract", wx.DefaultPosition, wx.DefaultSize, 0 ) 
CRE_sizer_5.Add( self.retract_dp_5, 0, wx.ALL|wx.ALIGN_CENTER_HORIZONTAL, 5 )

self.retract_dp_5.SetFont( wx.Font( 12, 74, 90, 90, False, "Calibri" ) )

self.extend_dp_5 = wx.RadioButton( self.CRE_panel_5, wx.ID_ANY, u"Extend", wx.DefaultPosition, wx.DefaultSize, 0 )

CRE_sizer_5.Addd (self.extend_dp_5, 0, wx.ALL|wx.ALIGN_CENTER_HORIZONTAL, 5 )

self.extend_dp_5.SetFont( wx.Font( 12, 74, 90, 90, False, "Calibri" ) )

self.CRE_panel_5.SetSizer(CRE_sizer_5 )

self.CRE_panel_5.Layout()

CRE_sizer_5.Fit( self.CRE_panel_5 )

param_dp_5.Add( self.CRE_panel_5, wx.GBPosition( 8,0 ), wx.GBSpan( 1,3 ), wx.EXPAND |wx.ALL, 5 )

fine_disp_sizer_5.Add( param_dp_5, 1, wx.ALIGN_CENTER_HORIZONTAL, 5 )

button_sizer_dp_5 = wx.BoxSizer(wx.VERTICAL )

self.indent_Button_dp 5 = wx.Button( self.fine_disp_panel 5, 29, u"Indent", wx.DefaultPosition, wx.Size( 175,60 ), 0 )

self.indent_Button_dp_5.SetFont( wx.Font( 18, 74, 90, 92, False, "Calibri" ) )

self.Bind(wx.EVT_BUTTON,self.displacementIndentExp,id=29)

button_sizer_dp_5.Add( self.indent_Button_dp_5, 0, wx.ALIGN_CENTER_HORIZONTAL|wx.ALL, 5 )

fine_disp_sizer_5.Add( button_sizer_dp_5, 1, wx.EXPAND, 5 )

self.m_panel77 = wx.Panel( self.fine_disp_panel_5, wx.ID_ANY, wx.DefaultPosition, wx.DefaultSize,wx.SIMPLE_BORDER )

gbSizer15 = wx.GridBagSizer $(0,0)$

gbSizer15.SetFlexibleDirection( wx.BOTH )

gbSizer15.SetNonFlexibleGrowMode( wx.FLEX_GROWMODE_SPECIFIED )

self.prior_disp_dp_5 = wx.StaticText( self.m_panel77, wx.ID_ANY, u"Prior displacements", wx.DefaultPosition, wx.DefaultSize, 0 ) self.prior_disp_dp_5.Wrap( -1 )

self.prior_disp_dp_5.SetFont( wx.Font( 14, 74, 90, 92, False, "Calibri" ) )

gbSizer15.Add( self.prior_disp_dp_5, wx.GBPosition( 0,0 ), wx.GBSpan( 1, 1 ), wx.ALL, 5 )

self.window_panel_dp_5 = wx.ScrolledWindow( self.m_panel77, wx.ID_ANY, wx.DefaultPosition, wx.DefaultSize,

wx.HSCROLL/wx.SIMPLE_BORDER/wx.VSCROLL )

self.window_panel_dp_5.SetScrollRate( 5, 5)

self.window_panel_dp_5.SetMinSize(wx.Size (320,350 ) )

window_sizer_dp_5 $=$ wx.FlexGridSizer $(0,7,0,0)$

window_sizer_dp_5.SetFlexibleDirection(wx.BOTH)

window_sizer_dp_5.SetNonFlexibleGrowMode( wx.FLEX_GROWMODE_SPECIFIED )

self.Disp_panel_dp_5 = wx.Panel( self.window_panel_dp_5, wx.ID_ANY, wx.DefaultPosition, wx.DefaultSize, wx.TAB_TRAVERSAL )

self.Disp_panel_dp_5.SetBackgroundColour(wx.SystemSettings.GetColour( wx.SYS_COLOUR_SCROLLBAR ) )

self.disp_sizer_dp_5 = wx.BoxSizer( wx.VERTICAL )

self.disp_sizer_dp_5.SetMinSize( wx.Size $(-1,350)$ )

self.disp_text_dp_5 = wx.StaticText( self.Disp_panel_dp_5, wx.ID_ANY, u"Displacement (mm)", wx.DefaultPosition, wx.Size( 100,30 ),

wx.ALIGN_CENTRE )

self.disp_text_dp_5.Wrap( -1 )

self.disp_text_dp_5.SetBackgroundColour(wx.SystemSettings.GetColour(wx.SYS_COLOUR_SCROLLBAR ) )

self.disp_sizer_dp_5.Add( self.disp_text_dp_5, 0, wx.TOP|wx.RIGHT|wx.LEFT, 5 )

self.disp_line_dp_5 $=$ wx.StaticLine ( self.Disp_panel_dp_5, wx.ID_ANY, wx.DefaultPosition, wx.DefaultSize, wx.LI_HORIZONTAL )

self.disp_sizer_dp_5.Add( self.disp_line_dp_5, 0, wx.EXPAND|wx.TOP|wx.BOTTOM, 5 )

self.Disp_panel_dp_5.SetSizer( self.disp_sizer_dp_5 )

self.Disp_panel_dp_5.Layout()

self.disp_sizer_dp_5.Fit( self.Disp_panel_dp_5 )

window_sizer_dp_5.Add( self.Disp_panel_dp_5, 1, wx.EXPAND|wx.BOTTOM, 5 )

self.velcity_panel_dp_5 = wx.Panel( self.window_panel_dp_5, wx.ID_ANY, wx.DefaultPosition, wx.DefaultSize, wx.TAB_TRAVERSAL )

self.velcity_panel_dp_5.SetBackgroundColour(wx.SystemSettings.GetColour(wx.SYS_COLOUR_3DLIGHT ) )

self.velcity_panel_dp_5.SetMinSize(wx.Size (100,350 ))

self.velocity_sizer_dp_5 = wx.BoxSizer(wx.VERTICAL )

self.velocity_sizer_dp_5.SetMinSize( wx.Size( 100,350) )

self.vel_text_dp_5 $=$ wx.StaticText( self.velcity_panel_dp_5, wx.ID_ANY, u"Velocity $\backslash n(\mathrm{~mm} / \mathrm{s})$ ", wx.DefaultPosition, wx.Size ( $-1,30)$,

wX.ALIGN_CENTRE )

self.vel_text_dp_5.Wrap ( -1 )

self.velocity_sizer_dp_5.Add( self.vel_text_dp_5, 0, wx.ALIGN_CENTER_HORIZONTAL|wx.TOP|wx.RIGHT|wx.LEFT, 5 )

self.vel_line_dp_5 = wx.StaticLine( self.velcity_panel_dp_5, wx.ID_ANY, wx.DefaultPosition, wx.DefaultSize, wx.LI_HORIZONTAL )

self.velocity_sizer_dp_5.Add( self.vel_line_dp_5, 0, wx.EXPAND|wx.TOP|wx.BOTTOM, 5 )

self.velcity_panel_dp_5.SetSizer(self.velocity_sizer_dp_5 )

self.velcity_panel_dp_5.Layout()

self.velocity_sizer_dp_5.Fit( self.velcity_panel_dp_5 )

window_sizer_dp_5.Add( self.velcity_panel_dp_5, 1, wx.EXPAND|wx.BOTTOM, 5 )

self.acceleration_panel_dp_5 =wx.Panel( self.window_panel_dp_5, wx.ID_ANY, wx.DefaultPosition, wx.DefaultSize, wx.TAB_TRAVERSAL )

self.acceleration_panel_dp_5.SetBackgroundColour(wx.SystemSettings.GetColour(wx.SYS_COLOUR_SCROLLBAR ) ) 
self.acceleration_panel_dp_5.SetMinSize( wx.Size (-1,350 ) )

self.acc_sizer_dp_5 = wx.BoxSizer( wx.VERTICAL )

self.acc_sizer_dp_5.SetMinSize( wx.Size $(100,350)$ )

self.acc_text_dp_5 $=$ wx.StaticText( self.acceleration_panel_dp_5, wx.ID_ANY, u"Acceleration $\backslash n(\mathrm{~mm} / \mathrm{s} / \mathrm{s}) "$, wx.DefaultPosition, wx.Size( $-1,30$

), wx.ALIGN_CENTRE)

self.acc_text_dp_5.Wrap( -1$)$

self.acc_sizer_dp_5.Add( self.acc_text_dp_5, 0, wx.ALIGN_CENTER_HORIZONTAL|wx.TOP|wx.RIGHT|wx.LEFT, 5 )

self.acc_line_dp_5 $=$ wx.StaticLine( self.acceleration_panel_dp_5, wx.ID_ANY, wx.DefaultPosition, wx.DefaultSize, wx.LI_HORIZONTAL )

self.acc_sizer_dp_5.Add( self.acc_line_dp_5, 0, wx.EXPAND|wx.TOP|wx.BOTTOM, 5 )

self.acceleration_panel_dp_5.SetSizer( self.acc_sizer_dp_5 )

self.acceleration_panel_dp_5.Layout()

self.acc_sizer_dp_5.Fit( self.acceleration_panel_dp_5 )

window_sizer_dp_5.Add( self.acceleration_panel_dp_5, 1, wx.EXPAND, 5 )

self.window_panel_dp_5.SetSizer( window_sizer_dp_5 )

self.window_panel_dp_5.Layout()

window_sizer_dp_5.Fit( self.window_panel_dp_5 )

gbSizer15.Add (self.window_panel_dp_5, wx.GBPosition( 1, 1), wx.GBSpan( 1,1 ), wx.EXPAND, 5 )

self.m_panel77.SetSizer( gbSizer15 )

self.m_panel77.Layout()

gbSizer15.Fit( self.m_panel77)

fine_disp_sizer_5.Add( self.m_panel77, 1, wx.EXPAND, 5 )

self.fine_disp_panel_5.SetSizer( fine_disp_sizer_5 )

self.fine_disp_panel_5.Layout()

fine_disp_sizer_5.Fit( self.fine_disp_panel_5 )

self.step5_disp_panel.AddPage( self.fine_disp_panel_5, u"Fine Displacement", True )

self.indentPlot_panel_5 = wx.Panel( self.step5_disp_panel, wx.ID_ANY, wx.DefaultPosition, wx.DefaultSize, wx.TAB_TRAVERSAL )

indentplot_sizer_5 $=$ wx.FlexGridSizer $(0,1,0,0)$

indentplot_sizer_5.SetFlexibleDirection(wx.BOTH )

indentplot_sizer_5.SetNonFlexibleGrowMode( wx.FLEX_GROWMODE_SPECIFIED )

self.select_plots_text_5 = wx.StaticText( self.indentPlot_panel_5, wx.ID_ANY, u"Select plots to view", wx.DefaultPosition, wx.Size( 660,-1 ), 0

)

self.select_plots_text_5.Wrap( -1$)$

self.select_plots_text_5.SetFont(wx.Font( 14, 74, 90, 92, False, "Calibri" ) )

indentplot_sizer_5.Add( self.select_plots_text_5, 0, wx.TOP|wx.BOTTOM|wx.RIGHT, 5 )

self.fine_disp_text_5 = wx.StaticText( self.indentPlot_panel_5, wx.ID_ANY, u"Fine displacement plots", wx.DefaultPosition, wx.DefaultSize, 0 )

self.fine_disp_text_5.Wrap( -1 )

self.fine_disp_text_5.SetFont( wx.Font( 14, 74, 90, 92, False, "Calibri" ) )

indentplot_sizer_5.Add( self.fine_disp_text_5,0,wx.RIGHT, 5 )

self.window_panel_fd_5 $=$ wx.ScrolledWindow( self.indentPlot_panel_5, wx.ID_ANY, wx.DefaultPosition, wx.DefaultSize,

wx.HSCROLL|wx.SIMPLE_BORDER|wx.VSCROLL )

self.window_panel_fd_5.SetScrollRate $(5,5)$

window_sizer_fd_ $5=$ wx.FlexGridSizer $(0,7,0,0)$

window_sizer_fd_5.SetFlexibleDirection(wx.BOTH )

window_sizer_fd_5.SetNonFlexibleGrowMode( wx.FLEX_GROWMODE_SPECIFIED )

self.window_panel_fd_5.SetMinSize(wx.Size( 400,300))

self.view_panel_fd_5 =wx.Panel( self.window_panel_fd_5, wx.ID_ANY, wx.DefaultPosition, wx.DefaultSize, wx.TAB_TRAVERSAL )

self.view_panel_fd_5.SetBackgroundColour(wx.SystemSettings.GetColour(wx.SYS_COLOUR_SCROLLBAR ) )

self.view_panel_fd_5.SetMinSize(wx.Size (65,300))

self.view_sizer_fd_5 = wx.BoxSizer( wx.VERTICAL )

self.view_sizer_fd_5.SetMinSize( wx.Size $(65,-1))$

self.view_text_fd_5 =wx.StaticText( self.view_panel_fd_5, wx.ID_ANY, u"View ", wx.DefaultPosition, wx.Size( 65,30 ),

wx.ALIGN_CENTRE )

self.view_text_fd_5.Wrap $(-1)$

self.view_text_fd_5.SetBackgroundColour(wx.SystemSettings.GetColour(wx.SYS_COLOUR_SCROLLBAR ) )

self.view_sizer_fd_5.Add( self.view_text_fd_5,0, wx.TOP|wx.RIGHT|wx.LEFT, 5 )

self.view_line_fd_5 =wx.StaticLine( self.view_panel_fd_5, wx.ID_ANY, wx.DefaultPosition, wx.DefaultSize, wx.LI_HORIZONTAL ) self.view_sizer_fd_5.Add( self.view_line_fd_5, 0, wx.EXPAND|wx.TOP|wx.BOTTOM, 5 )

self.view_panel_fd_5.SetSizer(self.view_sizer_fd_5 )

self.view_panel_fd_5.Layout()

self.view_sizer_fd_5.Fit( self.view_panel_fd_5 )

window_sizer_fd_5.Add( self.view_panel_fd_5, 1, wx.ALIGN_CENTER_HORIZONTAL|wx.EXPAND, 5 )

self.disp_panel_fd_5 = wx.Panel( self.window_panel_fd_5, wx.ID_ANY, wx.DefaultPosition, wx.DefaultSize, wx.TAB_TRAVERSAL )

self.disp_panel_fd_5.SetBackgroundColour(wx.SystemSettings.GetColour(wx.SYS_COLOUR_3DLIGHT ) )

self.disp_panel_fd_5.SetMinSize(wx.Size (100,300 )) 
self.disp_sizer_fd_5 = wx.BoxSizer(wx.VERTICAL )

self.disp_sizer_fd_5.SetMinSize( wx.Size (100,300) )

self.disp_text_fd_5 = wx.StaticText( self.disp_panel_fd_5, wx.ID_ANY, u"Displacement $\ln (\mathrm{mm})$ ", wx.DefaultPosition, wx.Size ( -1,30), wx.ALIGN_CENTRE )

self.disp_text_fd_5.Wrap( -1 )

self.disp_sizer_fd_5.Add( self.disp_text_fd_5, 0, wx.ALIGN_CENTER_HORIZONTAL|wx.TOP|wx.RIGHT|wx.LEFT, 5 )

self.disp_line_fd_5 = wx.StaticLine( self.disp_panel_fd_5, wx.ID_ANY, wx.DefaultPosition, wx.DefaultSize, wx.LI_HORIZONTAL ) self.disp_sizer_fd_5.Add( self.disp_line_fd_5, 0, wx.EXPAND|wx.TOP|wx.BOTTOM, 5 )

self.disp_panel_fd_5.SetSizer(self.disp_sizer_fd_5 )

self.disp_panel_fd_5.Layout()

self.disp_sizer_fd_5.Fit( self.disp_panel_fd_5 )

window_sizer_fd_5.Add( self.disp_panel_fd_5, 1, wx.EXPAND, 5 )

self.vel_panel_fd_5 = wx.Panel( self.window_panel_fd_5, wx.ID_ANY, wx.DefaultPosition, wx.DefaultSize, wx.TAB_TRAVERSAL )

self.vel_panel_fd_5.SetBackgroundColour( wx.SystemSettings.GetColour( wx.SYS_COLOUR_SCROLLBAR ) )

self.vel_panel_fd_5.SetMinSize (wx.Size $(-1,300))$

self.vel_sizer_fd_5 = wx.BoxSizer(wx.VERTICAL )

self.vel_sizer_fd_5.SetMinSize( wx.Size (100,300))

self.vel_text_fd_5 = wx.StaticText( self.vel_panel_fd_5, wx.ID_ANY, u"Velocityln(mm/s)", wx.DefaultPosition, wx.Size( $-1,30$ ),

wx.ALIGN_CENTRE )

self.vel_text_fd_5.Wrap ( -1$)$

self.vel_sizer_fd_5.Add( self.vel_text_fd_5, 0, wx.ALIGN_CENTER_HORIZONTAL|wx.TOP|wx.RIGHT|wx.LEFT, 5 )

self.vel_line_fd_5 = wx.StaticLine( self.vel_panel_fd_5, wx.ID_ANY, wx.DefaultPosition, wx.DefaultSize, wx.LI_HORIZONTAL )

self.vel_sizer_fd_5.Add( self.vel_line_fd_5, 0, wx.EXPAND|wx.TOP|wx.BOTTOM, 5 )

self.vel_panel_fd_5.SetSizer( self.vel_sizer_fd_5 )

self.vel_panel_fd_5.Layout()

self.vel_sizer_fd_5.Fit( self.vel_panel_fd_5 )

window_sizer_fd_5.Add( self.vel_panel_fd_5, 1, wx.EXPAND, 5 )

self.acc_panel_fd_5 = wx.Panel( self.window_panel_fd_5, wx.ID_ANY, wx.DefaultPosition, wx.DefaultSize, wx.TAB_TRAVERSAL )

self.acc_panel_fd_5.SetBackgroundColour(wx.SystemSettings.GetColour( wx.SYS_COLOUR_3DLIGHT ) )

self.acc_panel_fd_5.SetMinSize(wx.Size $(-1,300))$

self.acc_sizer_fd_5 =wx.BoxSizer(wx.VERTICAL )

self.acc_text_fd_5 =wx.StaticText( self.acc_panel_fd_5, wx.ID_ANY, u"Acceleration $\backslash n(\mathrm{~mm} / \mathrm{s} / \mathrm{s}) "$ ", wx.DefaultPosition, wx.Size ( 100,30 ), wX.ALIGN_CENTRE )

self.acc_text_fd_5.Wrap $(-1)$

self.acc_text_fd_5.SetBackgroundColour(wx.SystemSettings.GetColour(wx.SYS_COLOUR_3DLIGHT ) )

self.acc_sizer_fd_5.Add( self.acc_text_fd_5, 0, wx.TOP|wx.RIGHT|wx.LEFT, 5)

self.acc_line_fd_5 $=$ wx.StaticLine (self.acc_panel_fd_5, wx.ID_ANY, wx.DefaultPosition, wx.DefaultSize, wx.LI_HORIZONTAL )

self.acc_sizer_fd_5.Add( self.acc_line_fd_5, 0, wx.EXPAND|wx.TOP|wx.BOTTOM, 5 )

self.acc_panel_fd_5.SetSizer( self.acc_sizer_fd_5 )

self.acc_panel_fd_5.Layout()

self.acc_sizer_fd_5.Fit( self.acc_panel_fd_5 )

window_sizer_fd_5.Add( self.acc_panel_fd_5, 1, wx.EXPAND, 5 )

self.window_panel_fd_5.SetSizer(window_sizer_fd_5 )

self.window_panel_fd_5.Layout()

window_sizer_fd_5.Fit( self.window_panel_fd_5 )

indentplot_sizer_5.Add( self.window_panel_fd_5, 0, wx.BOTTOM|wx.RIGHT|wx.LEFT|wx.ALIGN_CENTER_HORIZONTAL, 5 )

self.view_button_fd_5 = wx.Button(self.indentPlot_panel_5, 30, u"View", wx.DefaultPosition, wx.Size (175,60 ), 0 )

self.view_button_fd_5.SetFont( wx.Font (18, 74, 90, 92, False, "Calibri" ) )

self.Bind(wx.EVT_BUTTON,self.viewDispPlots,id=30)

indentplot_sizer_5.Add( self.view_button_fd_5, 0, wx.ALL|wx.ALIGN_CENTER_HORIZONTAL, 5 )

self.indentPlot_panel_5.SetSizer( indentplot_sizer_5 )

self.indentPlot_panel_5.Layout()

indentplot_sizer_5.Fit( self.indentPlot_panel_5 )

self.step5_disp_panel.AddPage( self.indentPlot_panel_5, u"Indentation Plots", False )

decisionSizer.Add( self.step5_disp_panel, 1, wx.EXPAND, 5 )

self.decisionPanel.SetSizer( decisionSizer )

self.decisionPanel.Layout()

decisionSizer.Fit( self.decisionPanel )

decision_LogSizer.Add( self.decisionPanel, 1, wx.EXPAND|wx.TOP|wx.BOTTOM, 5 )

self.logPanel $=$ wx.Panel $($ self.decisionAndLogPanel, wx.ID_ANY, wx.DefaultPosition, wx.Size ( 550,600 ), wx.TAB_TRAVERSAL )

self.logPanel.SetBackgroundColour( wx.SystemSettings.GetColour( wx.SYS_COLOUR_INFOBK ) )

$\operatorname{logSizer}=w x$. FlexGridSizer $(5,1,0,0)$

$\operatorname{logSizer.SetFlexibleDirection(wx.BOTH})$

logSizer.SetNonFlexibleGrowMode( wx.FLEX_GROWMODE_SPECIFIED )

self.emergencyButtons = wx.Panel( self.logPanel, wx.ID_ANY, wx.DefaultPosition, wx.Size( 366,80), wx.TAB_TRAVERSAL )

embuttonsSizer $=$ wx.BoxSizer $($ wx.HORIZONTAL $)$

embuttonsSizer.SetMinSize( wx.Size( 366,80 ) )

self.emergencyStop = wx.Button( self.emergencyButtons, 31, u"Emergency ln Stop", wx.DefaultPosition, wx.Size( 175,70 ),

0|wx.RAISED_BORDER ) 
self.emergencyStop.SetFont( wx.Font( 18, 74, 90, 92, False, "Calibri" ) )

self.emergencyStop.SetForegroundColour( wx.SystemSettings.GetColour( wx.SYS_COLOUR_INACTIVEBORDER ) )

self.emergencyStop.SetBackgroundColour( wx.Colour $247,15,2$ ) )

\#self.Bind(wx.EVT_BUTTON,self.viewDispPlots,id=31)

embuttonsSizer.Add( self.emergencyStop, 0, wx.ALIGN_CENTER_VERTICAL|wx.ALL, 5 )

self.homeSled = wx.Button( self.emergencyButtons, 32, u"Home $\ln$ Sled", wx.DefaultPosition, wx.Size( 80,70 ), 0 )

self.homeSled.SetFont( wx.Font( 12, 74, 90, 90, False, "Calibri" ) )

self.Bind(wx.EVT_BUTTON,self.home,id=32)

embuttonsSizer.Add( self.homeSled, 0, wx.ALL|wx.ALIGN_CENTER_VERTICAL, 5 )

self.above_0 $=$ wx.Button( self.emergencyButtons, 33, u"20 mm In above P.O.C.", wx.DefaultPosition, wx.Size ( 80,70 ), 0 )

self.above_0.SetFont( wx.Font( 12, 74, 90, 90, False, "Calibri" ) )

self.Bind(wx.EVT_BUTTON,self.highestPoint,id=33)

embuttonsSizer.Add( self.above_0, 0, wx.ALL|wx.ALIGN_CENTER_VERTICAL, 5 )

self.emergencyButtons.SetSizer( embuttonsSizer )

self.emergencyButtons.Layout()

$\log$ Sizer.Add( self.emergencyButtons, 1, wx.EXPAND|wx.ALL, 5 )

self.force_Slider $=$ wx.Panel $($ self.logPanel, wx.ID_ANY, wx.DefaultPosition, wx.DefaultSize, wx.TAB_TRAVERSAL|wx.SIMPLE_BORDER )

self.force_Slider.SetMinSize( wx.Size( 350,80 ) )

force_Slider_sizer $=$ wx.BoxSizer $(w x$. VERTICAL $)$

force_Slider_sizer.SetMinSize( wx.Size $(350,80)$ )

wid = Widget(self.force_Slider,-1)

force_Slider_sizer.Add(wid,1,wx.EXPAND)

self.text_forceRead_label = wx.StaticText( self.force_Slider, wx.ID_ANY, "force (mN)", wx.DefaultPosition, wx.Size( $-1,-1)$,

wx.ALIGN_CENTRE )

self.text_forceRead_label.Wrap( -1 )

self.text_forceRead_label.SetFont(wx.Font( 12, 74, 90, 92, False, "Calibri" ) )

force_Slider_sizer.Add(self.text_forceRead_label,1,wx.ALIGN_CENTER_HORIZONTAL)

set_Potent $=$ wx.FlexGridSizer $(0,2,0,0)$

set_Potent.SetFlexibleDirection( wx.BOTH )

set_Potent.SetNonFlexibleGrowMode( wx.FLEX_GROWMODE_SPECIFIED )

self.text_setlcRange = wx.StaticText $($ self.force_Slider, wx.ID_ANY, "Set loadcell range (mN): ", wx.DefaultPosition, wx.Size ( $-1,-1)$,

wx.ALIGN_CENTRE )

self.text_setlcRange.Wrap ( -1$)$

self.text_setlcRange.SetFont( wx.Font( 12, 74, 90, 90, False, "Calibri" ) )

set_Potent.Add(self.text_setlcRange,1,wx.ALIGN_CENTER_HORIZONTAL)

potent_Val $=$ wx.GridSizer $(0,3,0,0)$

self.value_500 $=$ wx.RadioButton( self.force_Slider, wx.ID_ANY, u"500", wx.DefaultPosition, wx.DefaultSize, 0 )

potent_Val.Add( self.value_500, 0, wx.ALL|wx.ALIGN_CENTER_HORIZONTAL, 5 )

self.value_500.SetFont( wx.Font( 12, 74, 90, 90, False, "Calibri" ) )

self.value_1000 = wx.RadioButton( self.force_Slider, wx.ID_ANY, u"1000", wx.DefaultPosition, wx.DefaultSize, 0 )

potent_Val.Add( self.value_1000, 0, wx.ALL|wx.ALIGN_CENTER_HORIZONTAL, 5 )

self.value_1000.SetFont( wx.Font( 12, 74, 90, 90, False, "Calibri" ) )

self.value_1500 = wx.RadioButton ( self.force_Slider, wx.ID_ANY, u"1500", wx.DefaultPosition, wx.DefaultSize, 0 )

potent_Val.Add( self.value_1500, 0, wx.ALL|wx.ALIGN_CENTER_HORIZONTAL, 5 )

self.value_1500.SetFont( wx.Font( 12, 74, 90, 90, False, "Calibri" ) )

self.Bind(wx.EVT_RADIOBUTTON,self.setPotentVal1,self.value_1500)

self.Bind(wx.EVT_RADIOBUTTON,self.setPotentVal2,self.value_1000)

self.Bind(wx.EVT_RADIOBUTTON,self.setPotentVal3,self.value_500)

set_Potent.Add(potent_Val,1,wx.ALIGN_CENTER_HORIZONTAL)

force_Slider_sizer.Add(set_Potent,1,wx.EXPAND)

self.force_Slider.SetSizer(force_Slider_sizer )

self.force_Slider.Layout()

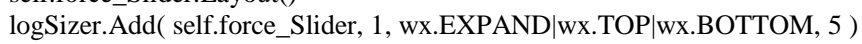

self.status_panel $=$ wx.Panel $($ self.logPanel, wx.ID_ANY, wx.DefaultPosition, wx.Size ( 416,80 ), 0 )

self.sizer_panel $=$ wx.GridBagSizer $(0,0)$

self.sizer_panel.SetFlexibleDirection( wx.BOTH )

self.sizer_panel.SetNonFlexibleGrowMode( wx.FLEX_GROWMODE_ALL ) 
\#sizer_panel.SetMinSize( wx.Size( 416,70 ) )

self.text_status $=$ wx.StaticText $($ self.status_panel, wx.ID_ANY, "Status:", wx.DefaultPosition, wx.Size( 100,30 ), wx.ALIGN_CENTRE $)$

self.text_status.Wrap( -1$)$

self.text_status.SetFont( wx.Font( 14, 74, 90, 92, False, "Calibri" ) )

self.text_status.SetBackgroundColour( wx.SystemSettings.GetColour( wx.SYS_COLOUR_WINDOW ) )

self.sizer_panel.Add( self.text_status, wx.GBPosition( 0, 0 ), wx.GBSpan( 1, 1 ), wx.TOP, 5 )

self.Status = wx.StaticText( self.status_panel, wx.ID_ANY, "Ready", wx.DefaultPosition, wx.Size( 70,30), wx.ALIGN_CENTRE )

self.Status.Wrap ( -1$)$

self.Status.SetFont( wx.Font( 16, 74, 90, 92, False, "Calibri" ) )

self.Status.SetForegroundColour( wx.Colour( 101, 218, 80 ) )

self.Status.SetBackgroundColour( wx.SystemSettings.GetColour( wx.SYS_COLOUR_WINDOW ) )

self.sizer_panel.Add( self.Status, wx.GBPosition( 0,1 ), wx.GBSpan( 1,1 ), wx.TOP, 5 )

self.text_for = wx.StaticText( self.status_panel, wx.ID_ANY, u" for ", wx.DefaultPosition, wx.Size( 40,30), wx.ALIGN_RIGHT )

self.text_for.Wrap (-1)

self.text_for.SetFont( wx.Font( 14, 74, 90, 92, False, "Calibri") )

self.text_for.SetBackgroundColour(wx.SystemSettings.GetColour(wx.SYS_COLOUR_WINDOW ) )

self.sizer_panel.Add( self.text_for, wx.GBPosition( 0,2 ), wx.GBSpan( 1,1$)$, wx.TOP, 5 )

self.time_status $=$ wx.StaticText( self.status_panel, wx.ID_ANY, u" hh:mm:ss", wx.DefaultPosition, wx.Size (200,30 ), wx.ALIGN_LEFT )

self.time_status.Wrap( -1$)$

self.time_status.SetFont(wx.Font( 14, 74, 90, 90, False, "Calibri" ))

self.time_status.SetBackgroundColour( wx.SystemSettings.GetColour( wx.SYS_COLOUR_3DLIGHT ))

self.timer $=$ wx.Timer(self)

self.global_log_start_time =datetime.datetime.now()

self.local_log_start_time = datetime.datetime.now()

self.Bind(wx.EVT_TIMER,self.update,self.timer)

self.sizer_panel.Add( self.time_status, wx.GBPosition( 0, 3 ), wx.GBSpan( 1, 1 ), wx.TOP, 5 )

self.text_logtime $=$ wx.StaticText( self.status_panel, wx.ID_ANY, u"Log Time: ", wx.DefaultPosition, wx.DefaultSize, 0 )

self.text_logtime.Wrap( -1$)$

self.text_logtime.SetFont( wx.Font( 14, 74, 90, 92, False, "Calibri" ) )

self.sizer_panel.Add( self.text_logtime, wx.GBPosition( 1, 1 ), wx.GBSpan( 1, 2 ), wx.ALIGN_RIGHT|wx.TOP|wx.BOTTOM, 5 )

self.time_log = wx.StaticText( self.status_panel, wx.ID_ANY, u" hh:mm:ss", wx.DefaultPosition, wx.Size( $150,-1$ ), 0 )

self.time_log.Wrap ( -1$)$

self.time_log.SetFont( wx.Font (14, 74, 90, 90, False, "Calibri" ) )

self.time_log.SetBackgroundColour( wx.SystemSettings.GetColour(wx.SYS_COLOUR_3DLIGHT ))

self.sizer_panel.Add( self.time_log, wx.GBPosition( 1,3 ), wx.GBSpan( 1,1$)$, wx.ALL, 5 )

self.status_panel.SetSizer( self.sizer_panel )

self.status_panel.Layout()

$\log$ Sizer.Add( self.status_panel, 1, wx.EXPAND|wx.RIGHT, 5 )

self.scrolled_log_panel $=$ wx.ScrolledWindow( self.logPanel, wx.ID_ANY, wx.DefaultPosition, wx.Size ( 416,300 ),

wx.HSCROLL/wx.SIMPLE_BORDER|wx.VSCROLL )

self.scrolled_log_panel.SetScrollRate $(5,5)$

$\log$ Data_sizer $=$ wx.FlexGridSizer $(0,7,0,0)$

logData_sizer.SetFlexibleDirection(wx.BOTH )

logData_sizer.SetNonFlexibleGrowMode( wx.FLEX_GROWMODE_SPECIFIED )

logData_sizer.SetMinSize( wx.Size (416,800))

self.type_panel $=$ wx.Panel $($ self.scrolled_log_panel, wx.ID_ANY, wx.DefaultPosition, wx.DefaultSize, wx.TAB_TRAVERSAL )

\# self.type_panel.SetMinSize $($ wx.Size $(-1)$,

self.type_panel.SetBackgroundColour(wx.SystemSettings.GetColour(wx.SYS_COLOUR_WINDOW ) )

self.type_sizer $=$ wx.BoxSizer $($ wx.VERTICAL $)$

self.type_sizer.SetMinSize( wx.Size( $-1,800)$ )

self.type_header $=$ wx.StaticText $($ self.type_panel, wx.ID_ANY, u"type", wx.DefaultPosition, wx.Size ( 50,30 ), wx.ALIGN_CENTRE )

self.type_header.Wrap $(-1)$

self.type_sizer.Add( self.type_header, 0, wx.ALL|wx.ALIGN_CENTER_HORIZONTAL, 5 )

self.type_staticline $=$ wx.StaticLine $($ self.type_panel, wx.ID_ANY, wx.DefaultPosition, wx.DefaultSize, wx.LI_HORIZONTAL )

self.type_sizer.Add( self.type_staticline, 0, wx.EXPAND|wx.TOP|wx.BOTTOM, 5 )

self.type_panel.SetSizer( self.type_sizer)

self.type_panel.Layout()

self.type_sizer.Fit( self.type_panel )

logData_sizer.Add( self.type_panel, 1, wx.EXPAND, 5 )

self.time_panel $=$ wx.Panel( self.scrolled_log_panel, wx.ID_ANY, wx.DefaultPosition, wx.DefaultSize, wx.TAB_TRAVERSAL )

self.time_panel.SetBackgroundColour(wx.SystemSettings.GetColour(wx.SYS_COLOUR_3DLIGHT) )

self.time_panel.SetMinSize( wx.Size ( $-1,800)$ )

self.time_sizer $=$ wx.BoxSizer $($ wx.VERTICAL $)$

self.time_sizer.SetMinSize( wx.Size( $-1,800)$ )

self.time_header = wx.StaticText( self.time_panel, wx.ID_ANY, u"timeln(hh:mm:ss)", wx.DefaultPosition, wx.Size( -1,30 ),

wx.ALIGN_CENTRE )

self.time_header.Wrap (-1)

self.time_sizer.Add( self.time_header, 0, wx.ALL|wx.ALIGN_CENTER_HORIZONTAL, 5 )

self.time_staticline $=$ wx.StaticLine $($ self.time_panel, wx.ID_ANY, wx.DefaultPosition, wx.DefaultSize, wx.LI_HORIZONTAL ) 
self.time_sizer.Add( self.time_staticline, 0, wx.EXPAND|wx.TOP|wx.BOTTOM, 5 )

self.time_panel.SetSizer( self.time_sizer )

self.time_panel.Layout()

self.time_sizer.Fit( self.time_panel )

logData_sizer.Add( self.time_panel, 1, wx.EXPAND, 5 )

self.disp_panel $=$ wx.Panel( self.scrolled_log_panel, wx.ID_ANY, wx.DefaultPosition, wx.DefaultSize, wx.TAB_TRAVERSAL )

self.disp_panel.SetBackgroundColour( wx.SystemSettings.GetColour( wx.SYS_COLOUR_WINDOW ) )

self.disp_panel.SetMinSize( wx.Size ( $-1,800)$ )

self.disp_sizer $=$ wx.BoxSizer( wx.VERTICAL $)$

self.disp_sizer.SetMinSize( wx.Size ( $-1,800)$ )

self.disp_header $=$ wx.StaticText( self.disp_panel, wx.ID_ANY, u"displn(mm)", wx.DefaultPosition, wx.Size( $-1,30$ ), wx.ALIGN_CENTRE )

self.disp_header.Wrap( -1 )

self.disp_sizer.Add( self.disp_header, 0, wx.ALL, 5 )

self.disp_staticline $=$ wx.StaticLine $($ self.disp_panel, wx.ID_ANY, wx.DefaultPosition, wx.DefaultSize, wx.LI_HORIZONTAL )

self.disp_sizer.Add( self.disp_staticline, 0, wx.EXPAND|wx.TOP|wx.BOTTOM, 5 )

self.disp_panel.SetSizer( self.disp_sizer )

self.disp_panel.Layout()

self.disp_sizer.Fit( self.disp_panel )

logData_sizer.Add( self.disp_panel, 1, wx.EXPAND, 5 )

self.force_panel $=$ wx.Panel $($ self.scrolled_log_panel, wx.ID_ANY, wx.DefaultPosition, wx.DefaultSize, wx.TAB_TRAVERSAL )

self.force_panel.SetBackgroundColour( wx.SystemSettings.GetColour(wx.SYS_COLOUR_3DLIGHT ) )

self.force_panel.SetMinSize( wx.Size ( $-1,800)$ )

self.force_sizer $=w x$. BoxSizer $(w x$. VERTICAL $)$

self.force_sizer.SetMinSize( wx.Size( $-1,800)$ )

self.force_header $=$ wx.StaticText( self.force_panel, wx.ID_ANY, u"forceln(mN)", wx.DefaultPosition, wx.Size( 40,30 ), wx.ALIGN_CENTRE )

self.force_header.Wrap( -1$)$

self.force_sizer.Add( self.force_header, 0, wx.ALL|wx.ALIGN_CENTER_HORIZONTAL, 5 )

self.force_staticline $=$ wx.StaticLine $($ self.force_panel, wx.ID_ANY, wx.DefaultPosition, wx.DefaultSize, wx.LI_HORIZONTAL )

self.force_sizer.Add( self.force_staticline, 0, wx.EXPAND|wx.TOP|wx.BOTTOM, 5 )

self.force_panel.SetSizer( self.force_sizer )

self.force_panel.Layout()

self.force_sizer.Fit( self.force_panel )

logData_sizer.Add( self.force_panel, 1, wx.EXPAND, 5 )

self.vel_panel $=$ wx.Panel $($ self.scrolled_log_panel, wx.ID_ANY, wx.DefaultPosition, wx.DefaultSize, wx.TAB_TRAVERSAL )

self.vel_panel.SetMinSize( wx.Size $(-1,800)$ )

self.vel_panel.SetBackgroundColour(wx.SystemSettings.GetColour(wx.SYS_COLOUR_WINDOW ) )

self.vel_sizer $=$ wx.BoxSizer $($ wx.VERTICAL $)$

self.vel_sizer.SetMinSize( wx.Size( $-1,800)$ )

self.vel_header = wx.StaticText $($ self.vel_panel, wx.ID_ANY, u"vel $\backslash n(\mathrm{~mm} / \mathrm{s}) "$, wx.DefaultPosition, wx.Size( 50,30 ), wx.ALIGN_CENTRE )

self.vel_header.Wrap( -1 )

self.vel_sizer.Add( self.vel_header, 0 , wx.ALL, 5 )

self.vel_staticline = wx.StaticLine( self.vel_panel, wx.ID_ANY, wx.DefaultPosition, wx.DefaultSize, wx.LI_HORIZONTAL )

self.vel_sizer.Add( self.vel_staticline, 0, wx.EXPAND|wx.TOP|wx.BOTTOM, 5 )

self.vel_panel.SetSizer( self.vel_sizer)

self.vel_panel.Layout()

self.vel_sizer.Fit( self.vel_panel )

logData_sizer.Add( self.vel_panel, 1, wx.EXPAND, 5 )

self.acc_panel = wx.Panel( self.scrolled_log_panel, wx.ID_ANY, wx.DefaultPosition, wx.DefaultSize, wx.TAB_TRAVERSAL )

self.acc_panel.SetBackgroundColour( wx.SystemSettings.GetColour( wx.SYS_COLOUR_3DLIGHT ) )

self.acc_panel.SetMinSize( wx.Size( $-1,800)$ )

self.acc_sizer $=$ wx.BoxSizer( wx.VERTICAL $)$

self.acc_sizer.SetMinSize( wx.Size ( $-1,800)$ )

self.acc_header $=$ wx.StaticText $($ self.acc_panel, wx.ID_ANY, u"acc $\backslash n(\mathrm{~mm} / \mathrm{s} / \mathrm{s}) "$, wx.DefaultPosition, wx.Size( 50,30), wx.ALIGN_CENTRE $)$

self.acc_header.Wrap (-1)

self.acc_sizer.Add( self.acc_header, 0, wx.ALL|wx.ALIGN_CENTER_HORIZONTAL, 5 )

self.acc_staticline $=$ wx.StaticLine $($ self.acc_panel, wx.ID_ANY, wx.DefaultPosition, wx.DefaultSize, wx.LI_HORIZONTAL $)$

self.acc_sizer.Add( self.acc_staticline, 0, wx.EXPAND|wx.TOP|wx.BOTTOM, 5 )

self.acc_panel.SetSizer( self.acc_sizer )

self.acc_panel.Layout()

self.acc_sizer.Fit( self.acc_panel )

logData_sizer.Add( self.acc_panel, 1, wx.EXPAND, 5 )

self.hold_panel = wx.Panel( self.scrolled_log_panel, wx.ID_ANY, wx.DefaultPosition, wx.DefaultSize, wx.TAB_TRAVERSAL )

self.hold_panel.SetMinSize( wx.Size (45,800))

self.hold_panel.SetBackgroundColour(wx.SystemSettings.GetColour( wx.SYS_COLOUR_WINDOW ))

self.hold_sizer $=$ wx.BoxSizer $($ wx.VERTICAL $)$

self.hold_sizer.SetMinSize( wx.Size( 45,800))

self.hold_header = wx.StaticText $($ self.hold_panel, wx.ID_ANY, u"hold $\ln (\mathrm{s}) "$ ", wx.DefaultPosition, wx.Size( 40,30), wx.ALIGN_CENTRE )

self.hold_header.Wrap $(-1)$ 
self.hold_sizer.Add( self.hold_header, 0, wx.TOP|wx.BOTTOM|wx.ALIGN_CENTER_HORIZONTAL, 5 )

self.hold_staticline $=w x$.StaticLine $($ self.hold_panel, wx.ID_ANY, wx.DefaultPosition, wx.DefaultSize, wx.LI_HORIZONTAL )

self.hold_sizer.Add( self.hold_staticline, 0, wx.EXPAND|wx.TOP|wx.BOTTOM, 5 )

self.hold_panel.SetSizer( self.hold_sizer )

self.hold_panel.Layout()

self.hold_sizer.Fit( self.hold_panel )

logData_sizer.Add( self.hold_panel, 1, wx.EXPAND, 5 )

self.scrolled_log_panel.SetSizer( $\log$ Data_sizer )

self.scrolled_log_panel.Layout()

$\log$ Sizer.Add( self.scrolled_log_panel, 1, wx.EXPAND, 5 )

self.mouse_exp_ID = wx.Panel( self.logPanel, wx.ID_ANY, wx.DefaultPosition, wx.Size ( 416,100 ), wx.TAB_TRAVERSAL )

mouse_exp_ID_sizer $=w x$.GridSizer $(2,2,0,0)$

self.text_MouseId $=$ wx.StaticText( self.mouse_exp_ID, wx.ID_ANY, u"Mouse ID: ", wx.DefaultPosition, wx.DefaultSize, 0 )

self.text_MouseId.Wrap( -1$)$

self.text_MouseId.SetFont( wx.Font( 12, 74, 90, 92, False, "Calibri" ) )

mouse_exp_ID_sizer.Add( self.text_MouseId, 0, wx.ALL|wx.ALIGN_RIGHT, 5 )

self.mouse_ID = wx.TextCtrl( self.mouse_exp_ID, 10000, u"ID", wx.DefaultPosition, wx.DefaultSize,

wx.TE_CENTRE|wx.TE_PROCESS_ENTER )

self.mouse_ID.SetFont( wx.Font( 12, 74, 90, 90, False, "Calibri" ) )

self.Bind(wx.EVT_TEXT_ENTER, self.UpdateLogFile_mouse,id=10000)

mouse_exp_ID_sizer.Add( self.mouse_ID, 0, wx.BOTTOM|wx.RIGHT|wx.LEFT, 5 )

self.text_ExperimentalID = wx.StaticText( self.mouse_exp_ID, wx.ID_ANY, u"Experimental ID: ", wx.DefaultPosition, wx.DefaultSize, wx.TE_CENTRE )

self.text_ExperimentalID.SetFont( wx.Font( 12, 74, 90, 92, False, "Calibri" ) )

mouse_exp_ID_sizer.Add( self.text_ExperimentalID, 0, wx.ALL|wx.ALIGN_RIGHT, 5 )

self.experimental_ID = wx.TextCtrl( self.mouse_exp_ID, 9999, u"ID", wx.DefaultPosition, wx.DefaultSize,

wx.TE_CENTRE|wx.TE_PROCESS_ENTER )

self.experimental_ID.SetFont( wx.Font( 12, 74, 90, 90, False, "Calibri" ) )

self.Bind(wx.EVT_TEXT_ENTER, self.UpdateLogFile_Exp,id=9999)

mouse_exp_ID_sizer.Add( self.experimental_ID, 0, wx.BOTTOM|wx.RIGHT|wx.LEFT, 5 )

self.mouse_exp_ID.SetSizer( mouse_exp_ID_sizer )

self.mouse_exp_ID.Layout()

logSizer.Add( self.mouse_exp_ID, 1, wx.EXPAND |wx.ALL, 5 )

\#

self.logPanel.SetSizer( $\log$ Sizer )

self.logPanel.Layout()

decision_LogSizer.Add( self.logPanel, 1, wx.EXPAND|wx.ALL, 5 )

self.decisionAndLogPanel.SetSizer(decision_LogSizer )

self.decisionAndLogPanel.Layout()

topandBottomSizer.Add( self.decisionAndLogPanel, 1, wx.EXPAND|wx.RIGHT|wx.LEFT, 5 )

self.UI_Panel.SetSizer( topandBottomSizer )

self.UI_Panel.Layout()

topandBottomSizer.Fit( self.UI_Panel )

UISizer.Add( self.UI_Panel, 0, wx.EXPAND, 5 )

self.SetSizer( UISizer )

self.Layout()

self.Centre( wx.BOTH )

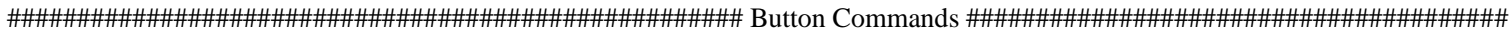

save_log_dialogFileName = "VirginiaIndenterLog_"+datetime.datetime.now().strftime("\%Y-\%m-\%d")+ "_" +

datetime.datetime.now().strftime("\%H_\%M_\%S")

self.timer.Start(1000)

potent_Mode $=$ loadCell $($ )

pub.subscribe(self.OnNewCW, "cw")

pub.subscribe(self.OnNewDisp, "Disp_Val")

$\log \_$started_string $=$" $\{$Log started at " +datetime.datetime.now().strftime("\%H:\%M:\%S") + " on "+datetime.datetime.now().strftime("\%Y-\%m$\left.\left.\% \mathrm{~d}^{\prime}\right)+"\right\} \backslash \mathrm{n} "$

Mouseid_string = "Mouse ID:__ _ + "In" + "Experimental ID:

FILE = open(fileLoc+'ll'+ save_log_dialogFileName+'.txt',"a")

FILE.write(log_started_string+"|n"+Mouseid_string+"|n")

self.Show()

def replace(self,file_path, pattern, subst):

\#Create temp file

fh, abs_path $=$ mkstemp()

new_file $=$ open (abs_path,'w')

old_file $=$ open(file_path) 
for line in old_file:

new_file.write(line.replace(pattern, subst))

\#close temp file

new_file.close()

close(fh)

old_file.close()

\#Remove original file

remove(file_path)

\#Move new file

move(abs_path, file_path)

def UpdateLogFile_mouse(self,event):

global fileLoc

global save_log_dialogFileName

mouseId = self.mouse_ID.GetValue()

self.replace(fileLoc+'II'+ save_log_dialogFileName+'.txt',"Mouse ID:__","Mouse ID: " +mouseId )

def UpdateLogFile_Exp(self,event):

global fileLoc

global save_log_dialogFileName

experimentalId = self.experimental_ID.GetValue ()

self.replace(fileLoc+' 'l'+ save_log_dialogFileName+'.txt',"Experimental ID: ","Experimental ID: " +experimentalId ) def OnNewCW(self,cw_update):

global cw

$\mathrm{cW}=\mathrm{cw} \_$update

wid.Refresh()

def OnNewDisp(self,disp_update):

global cw

global setMin

global setMax

global displayMinDisp_box_2

global displayMinForce_box_2

global dispMaxDisp_box_2

global dispMaxForce_box_2

$\mathrm{cw}=$ disp_update $[0]$

displacement $=$ disp_update[1]

if (not(setMin) and not(setMax) ):

displayMinDisp_box_2.SetValue((str('\{0:.2f\}'.format(displacement))))

displayMinForce_box_2.SetValue(str('\{0:.2f\}'.format $((\mathrm{cw}))))$

elif(setMin and not(setMax) ):

dispMaxDisp_box_2.SetValue(str('\{0:.2f\}'.format(displacement)))

dispMaxForce_box_2.SetValue(str('\{0:.2f\}'.format $(\mathrm{cw})))$

wid.Refresh()

def changePagesRight(self,event):

current_Step $=$ self.upperStep_text.GetLabel()[5]

if $($ current_Step $==$ ' 1 ')

global dispMode

global Disp_Mode

global setMin

global setMax

global Alone

global Disp_Mode

Alone $=$ False

self.upperStep_text.SetLabel("Step 2: Find Minimum and In Maximum Displacement")

self.Step2_Right.Hide()

self.Step2_Right.Disable()

self.NoButton_Left.Hide()

self.NoButton_Left.Disable()

self.Step1_Left.Show()

self.Step1_Left.Enable()

self.Step3_Right.Show()

self.Step3_Right.Enable(True)

self.step1_panel.Hide()

self.step1_panel.Disable()

self.step2_panel.Show()

self.step2_panel.Enable(True)

dispMode $=$ True

setMin $=$ False

setMax $=$ False

Disp_Mode $=$ DispThread $($ )

self.headerPanel.Layout()

self.Layout() 


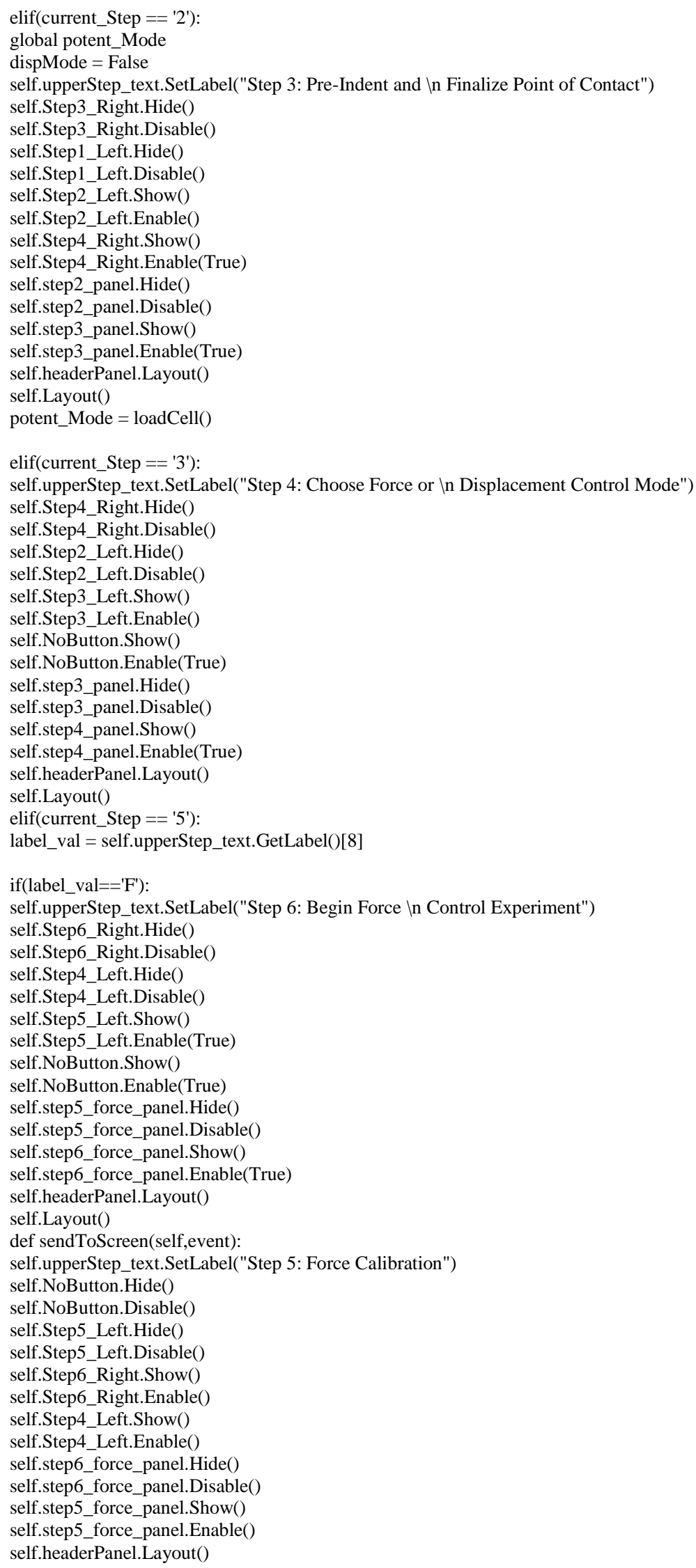




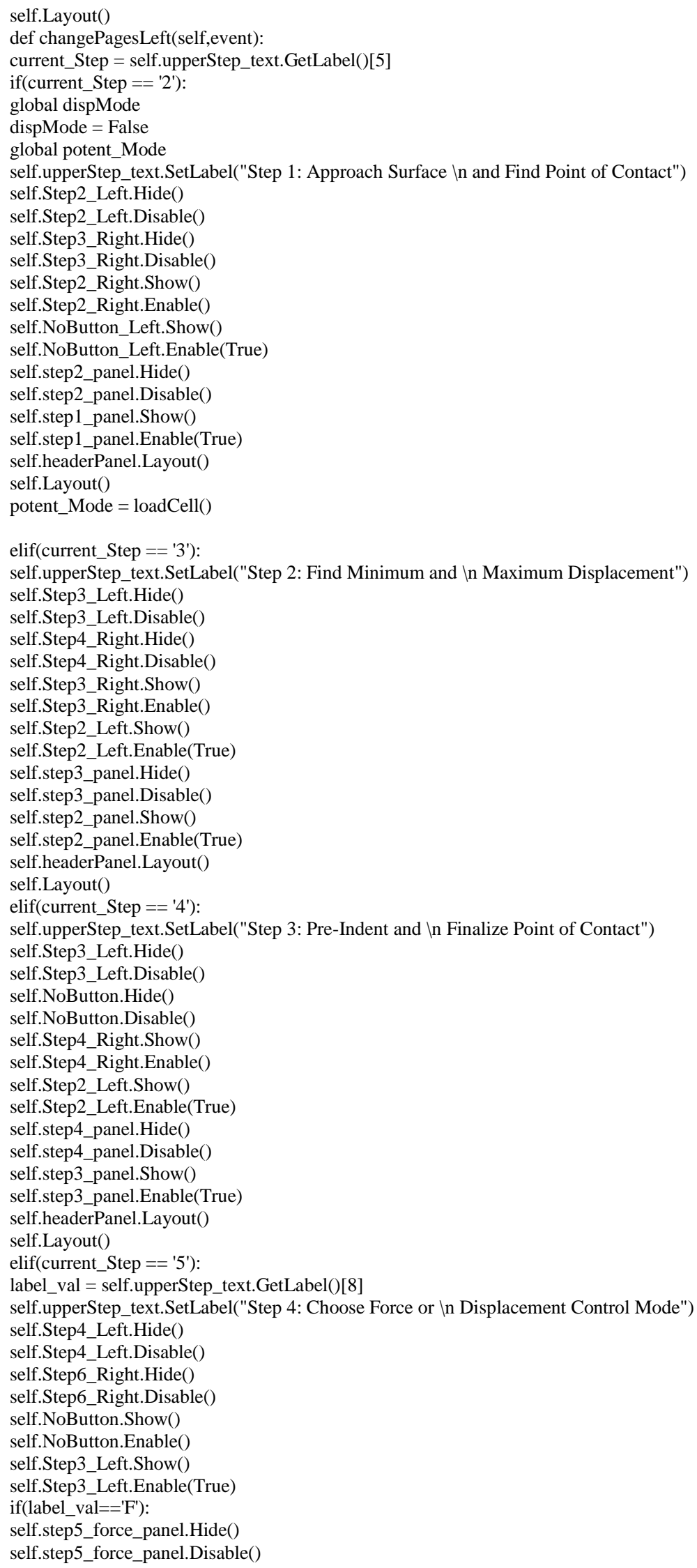


else:

self.step5_disp_panel.Hide()

self.step5_disp_panel.Disable()

self.step4_panel.Show()

self.step4_panel.Enable(True)

self.headerPanel.Layout()

self.Layout()

elif(current_Step == '6'):

self.upperStep_text.SetLabel("Step 5: Force Calibration")

self.NoButton.Hide()

self.NoButton.Disable()

self.Step5_Left.Hide()

self.Step5_Left.Disable()

self.Step6_Right.Show()

self.Step6_Right.Enable()

self.Step4_Left.Show()

self.Step4_Left.Enable()

self.step6_force_panel.Hide()

self.step6_force_panel.Disable()

self.step5_force_panel.Show()

self.step5_force_panel.Enable()

self.headerPanel.Layout()

self.Layout()

def change_forcePage(self,event):

self.upperStep_text.SetLabel("Step 5: Force Calibration")

self.Step3_Left.Hide()

self.Step3_Left.Disable()

self.NoButton.Hide()

self.NoButton.Disable()

self.Step4_Left.Show()

self.Step4_Left.Enable()

self.Step6_Right.Show()

self.Step6_Right.Enable(True)

self.step5_force_panel.Show()

self.step5_force_panel.Enable(True)

self.step4_panel.Hide()

self.step4_panel.Disable()

self.headerPanel.Layout()

self.Layout()

def change_dispPage(self,event):

self.upperStep_text.SetLabel("Step 5: Begin Displacement In Controlled Indentation")

self.Step3_Left.Hide()

self.Step3_Left.Disable()

self.NoButton.Hide()

self.NoButton.Disable()

self.Step4_Left.Show()

self.Step4_Left.Enable(True)

self.NoButton.Show()

self.NoButton.Enable(True)

self.step4_panel.Hide()

self.step4_panel.Disable()

self.step5_disp_panel.Show()

self.step5_disp_panel.Enable(True)

self.headerPanel.Layout()

self.Layout()

def changeMult_Single(self,event):

if(self.Calibrate_level_choice.GetString(self.Calibrate_level_choice.GetSelection())[0]=='M'):

self.calibration_param_5_panel.Hide()

self.calibration_param_5_panel.Disable()

self.calib_param_mutliple_5.Show()

self.calib_param_mutliple_5.Enable(True)

self.Layout()

else:

self.calib_param_mutliple_5.Hide()

self.calib_param_mutliple_5.Disable()

self.calibration_param_5_panel.Show()

self.calibration_param_5_panel.Enable(True)

self.Layout() 


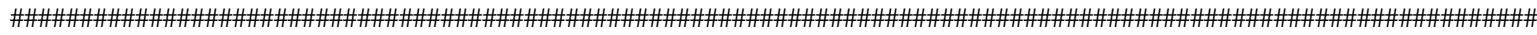

\#

\# Log Commands, Table Updates

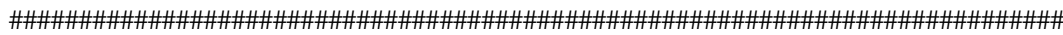

def destroyTables(self):

valueLength $=$ len(self.F_D_ID_sizer_6.GetChildren ()$)$

if self.F_D_ID_sizer_6.GetChildren():

for $\mathrm{x}$ in range( 0 ,valueLength- 2$)$ :

self.F_D_ID_sizer_6.Hide(valueLength-x-1)

self.F_D_ID_sizer_6.Remove(valueLength-X-1)

self.F_D_ID_panel_6.Layout()

self.F_D_force_sizer_6.Hide(valueLength-X-1)

self.F_D_force_sizer_6.Remove(valueLength-X-1)

self.F_D_force_panel_6.Layout()

self.F_D_disp_sizer_6.Hide(valueLength-X-1)

self.F_D_disp_sizer_6.Remove(valueLength-X-1)

self.F_D_disp_panel_6.Layout()

self.F_D_vel_sizer_6.Hide(valueLength-x-1)

self.F_D_vel_sizer_6.Remove(valueLength-x-1)

self.F_D_vel_panel_6.Layout()

self.calib_ID_sizer_5.Hide(valueLength-X-1)

self.calib_ID_sizer_5.Remove(valueLength-X-1)

self.calib_ID_panel_5.Layout()

self.calib_force_sizer_5.Hide(valueLength-X-1)

self.calib_force_sizer_5.Remove(valueLength-X-1)

self.calib_force_panel_5.Layout()

self.calib_disp_sizer_5.Hide(valueLength-X-1)

self.calib_disp_sizer_5.Remove(valueLength-X-1)

self.calib_disp_panel_5.Layout()

self.calib_vel_sizer_5.Hide(valueLength-x-1)

self.calib_vel_sizer_5.Remove(valueLength-x-1)

self.calib_vel_panel_5.Layout()

valueLength $=$ len $($ self.F_D_force_sizer_6.GetChildren () )

if self.F_D_force_sizer_6.GetChildren():

for $x$ in range $(0$, valueLength - 2$)$ :

self.F_D_force_sizer_6.Hide(valueLength-X-1)

self.F_D_force_sizer_6.Remove(valueLength-X-1)

self.F_D_force_panel_6.Layout()

def forceControlTableWrite (self,ID,disp,vel,force):

IDLog = wx.StaticText $($ self.calib_ID_panel_5, -1, str(ID))

self.calib_ID_sizer_5.Add( IDLog, 0, wx.ALL|wx.ALIGN_CENTER_HORIZONTAL, 5 )

self.calib_ID_panel_5.Layout()

self.calib_ID_sizer_5.Fit( self.calib_ID_panel_5)

ForceLog = wx.StaticText( self.calib_force_panel_5, -1, str(force))

self.calib_force_sizer_5.Add(ForceLog, 0, wx.ALL|wx.ALIGN_CENTER_HORIZONTAL, 5 )

self.calib_force_panel_5.Layout()

self.calib_force_sizer_5.Fit( self.calib_force_panel_5)

dispLog $=$ wx.StaticText $($ self.calib_disp_panel_5, -1, str(disp)

self.calib_disp_sizer_5.Add( dispLog, 0, wx.ALL|wx.ALIGN_CENTER_HORIZONTAL, 5 )

self.calib_disp_panel_5.Layout()

self.calib_disp_sizer_5.Fit( self.calib_disp_panel_5 )

velLog = wx.StaticText ( self.calib_vel_panel_5, -1, str(vel))

self.calib_vel_sizer_5.Add( velLog, 0, wx.ALL|wx.ALIGN_CENTER_HORIZONTAL, 5 )

self.calib_vel_panel_5.Layout()

self.calib_vel_sizer_5.Fit( self.calib_vel_panel_5)

IDLog_1 = wx.StaticText( self.F_D_ID_panel_6, -1, str(ID))

self.F_D_ID_sizer_6.Add( IDLog_1, 0, wx.ALL|wx.ALIGN_CENTER_HORIZONTAL, 5 )

self.F_D_ID_panel_6.Layout()

self.F_D_ID_sizer_6.Fit( self.F_D_ID_panel_6 )

ForceLog_1 $=$ wx.StaticText ( self.F_D_force_panel_6, 20000+ID, str(force))

self.F_D_force_sizer_6.Add(ForceLog_1, 0, wx.ALL|wx.ALIGN_CENTER_HORIZONTAL, 5 )

self.F_D_force_panel_6.Layout()

self.F_D_force_sizer_6.Fit( self.F_D_force_panel_6)

dispLog_1 = wx.StaticText ( self.F_D_disp_panel_6, 21000+ID, $\operatorname{str}(\operatorname{disp}))$

self.F_D_disp_sizer_6.Add( dispLog_1, 0, wx.ALL|wx.ALIGN_CENTER_HORIZONTAL, 5 )

self.F_D_disp_panel_6.Layout()

self.F_D_disp_sizer_6.Fit( self.F_D_disp_panel_6)

velLog_1 = wx.StaticText ( self.F_D_vel_panel_6, 22000+ID, $\operatorname{str}(\mathrm{vel})$ ) 
self.F_D_vel_sizer_6.Add( velLog_1, 0, wx.ALL|wx.ALIGN_CENTER_HORIZONTAL, 5 )

self.F_D_vel_panel_6.Layout()

self.F_D_vel_sizer_6.Fit( self.F_D_vel_panel_6)

def dispControlTableWrite (self,disp,vel,acc,var):

if $(\operatorname{var}==$ 'd'):

dispLog $=$ wx.StaticText $($ self.Disp_panel_dp_5, $-1, \operatorname{str}($ disp $))$

self.disp_sizer_dp_5.Add( dispLog, 0, wx.ALL|wx.ALIGN_CENTER_HORIZONTAL, 5 )

self.Disp_panel_dp_5.Layout()

self.disp_sizer_dp_5.Fit( self.Disp_panel_dp_5 )

velLog = wx.StaticText $($ self.velcity_panel_dp_5, $-1, \operatorname{str}($ vel $)$ )

self.velocity_sizer_dp_5.Add( velLog, 0, wx.ALL|wx.ALIGN_CENTER_HORIZONTAL, 5 )

self.velcity_panel_dp_5.Layout()

self.velocity_sizer_dp_5.Fit( self.velcity_panel_dp_5 )

accLog $=$ wx.StaticText $($ self.acceleration_panel_dp_5, -1, $\operatorname{str}(\mathrm{acc}))$

self.acc_sizer_dp_5.Add( accLog, 0, wx.ALL|wx.ALIGN_CENTER_HORIZONTAL, 5 )

self.acceleration_panel_dp_5.Layout()

self.acc_sizer_dp_5.Fit( self.acceleration_panel_dp_5 )

else:

dispLog = wx.StaticText( self.Disp_panel_6, -1, str(disp))

self.disp_sizer11.Add( dispLog, 0, wx.ALL|wx.ALIGN_CENTER_HORIZONTAL, 5 )

self.Disp_panel_6.Layout()

self.disp_sizer11.Fit( self.Disp_panel_6)

velLog = wx.StaticText $($ self.velcity_panel_6, $-1, \operatorname{str}(v e l))$

self.velocity_sizer11.Add( velLog, 0, wx.ALL|wx.ALIGN_CENTER_HORIZONTAL, 5 )

self.velcity_panel_6.Layout()

self.velocity_sizer11.Fit( self.velcity_panel_6)

accLog $=$ wx.StaticText $($ self.acceleration_panel_6, $-1, \operatorname{str}(\operatorname{acc}))$

self.acc_sizer11.Add( accLog, 0, wx.ALL|wx.ALIGN_CENTER_HORIZONTAL, 5 )

self.acceleration_panel_6.Layout()

self.acc_sizer11.Fit( self.acceleration_panel_6 )

def dispViewTableWrite (self,disp,vel,acc,val):

global checkBox_ID

global cb_list

global checkBox_ID_f

global cb_list_f

if $(\mathrm{val}==\mathbf{d}$ '):

viewLog $=$ wx.CheckBox(self.view_panel_fd_5,checkBox_ID)

cb_list.append([viewLog,disp,vel,acc])

\#wx.EVT_CHECKBOX(self,checkBox_ID,self.dispBoxClick)

self.view_sizer_fd_5.Add(viewLog,0,wx.ALL|wx.ALIGN_CENTER_HORIZONTAL, 5 )

self.view_panel_fd_5.Layout()

self.view_sizer_fd_5.Fit( self.view_panel_fd_5 )

dispLog $=$ wx.StaticText( self.disp_panel_fd_5, -1, str(disp))

self.disp_sizer_fd_5.Add( dispLog, 0, wx.ALL|wx.ALIGN_CENTER_HORIZONTAL, 5 )

self.disp_panel_fd_5.Layout()

self.disp_sizer_fd_5.Fit( self.disp_panel_fd_5 )

velLog = wx.StaticText( self.vel_panel_fd_5, $-1, \operatorname{str}($ vel $))$

self.vel_sizer_fd_5.Add( velLog, 0, wx.ALL|wx.ALIGN_CENTER_HORIZONTAL, 5 )

self.vel_panel_fd_5.Layout()

self.vel_sizer_fd_5.Fit( self.vel_panel_fd_5 )

$\operatorname{accLog}=$ wx.StaticText $($ self.acc_panel_fd_5, $-1, \operatorname{str}(\operatorname{acc}))$

self.acc_sizer_fd_5.Add( accLog, 0, wx.ALL|wx.ALIGN_CENTER_HORIZONTAL, 5 )

self.acc_panel_fd_5.Layout()

self.acc_sizer_fd_5.Fit( self.acc_panel_fd_5 )

checkBox_ID $=$ checkBox_ID+1

else:

viewLog = wx.CheckBox(self.type_panel_fd_6,checkBox_ID_f)

cb_list_f.append([viewLog,disp,vel,acc])

\#wx.EVT_CHECKBOX(self,checkBox_ID,self.dispBoxClick)

self.type_sizer_fd_6.Add(viewLog,0,wx.ALL|wx.ALIGN_CENTER_HORIZONTAL, 5 )

self.type_panel_fd_6.Layout()

self.type_sizer_fd_6.Fit( self.type_panel_fd_6)

dispLog $=$ wx.StaticText $($ self.disp_panel_fd_6, $-1, \operatorname{str}(\operatorname{disp}))$

self.disp_sizer_fd_6.Add( dispLog, 0, wx.ALL|wx.ALIGN_CENTER_HORIZONTAL, 5 )

self.disp_panel_fd_6.Layout()

self.disp_sizer_fd_6.Fit( self.disp_panel_fd_6 )

velLog $=$ wx.StaticText( self.vel_panel_fd_6, $-1, \operatorname{str}(\mathrm{vel}))$

self.vel_sizer_fd_6.Add( velLog, 0, wx.ALL|wx.ALIGN_CENTER_HORIZONTAL, 5 )

self.vel_panel_fd_6.Layout()

self.vel_sizer_fd_6.Fit( self.vel_panel_fd_6 ) 
accLog $=w x . S t a t i c T e x t($ self.acc_panel_fd_6, $-1, \operatorname{str}(\operatorname{acc}))$

self.acc_sizer_fd_6.Add( accLog, 0, wx.ALL|wx.ALIGN_CENTER_HORIZONTAL, 5 )

self.acc_panel_fd_6.Layout()

self.acc_sizer_fd_6.Fit( self.acc_panel_fd_6 )

checkBox_ID_f $=$ checkBox_ID_f +1

def forceViewArrayWrite (self,Force,disp,vel,f0d,checkBox_ID_val):

\#\#\#ID in 5,000, forces as value 5010 displacement 5011

global cb_list_Values

global presentImages

sizeMove $=$ len (presentImages)

isAVal $=$ False

ForceBool $=$ False

if(fOd):

for $\mathrm{x}$ in range( 0 , sizeMove):

ForceBool $=$ True

if(presentImages $[\mathrm{x}][0][0]==$ Force and presentImages $[\mathrm{x}][0][1]==\mathrm{vel}$ and not(presentImages[x][0][3])):

isAVal $=$ True

presentImages $[\mathrm{x}][0][3]=$ True

presentImages[x][0].append(checkBox_ID_val)

ForceLog = wx.CheckBox(self.type_panel_f_d_6,checkBox_ID_val)

cb_list_Values.append([ForceLog,Force,vel,disp,ForceBool])

elif(presentImages[x][0][0]==Force and $\operatorname{not}(\operatorname{presentImages}[\mathrm{x}][0][1]==\mathrm{vel})$ ):

isAVal $=$ True

presentImages.append([[Force,vel,disp,True,checkBox_ID_val],[Force,vel,disp,False]])

ForceLog = wx.CheckBox(self.type_panel_f_d_6,checkBox_ID_val)

cb_list_Values.append([ForceLog,Force,vel,disp,ForceBool] $)$

elif(presentImages $[\mathrm{x}][0][0]==$ Force and presentImages $[\mathrm{x}][0][1]==\mathrm{vel}$ and presentImages $[\mathrm{x}][0][3])$ :

isAVal $=$ True

presentImages $[\mathrm{x}][0]=[$ Force,vel,disp,True,checkBox_ID_val $]$

ForceLog = wx.CheckBox(self.type_panel_f_d_6,checkBox_ID_val)

cb_list_Values.append([ForceLog,Force,vel,disp,ForceBool] $)$

if(not(isAVal)):

presentImages.append([[Force,vel,disp,True,checkBox_ID_val],[Force,vel,disp,False]])

ForceLog = wx.CheckBox(self.type_panel_f_d_6,checkBox_ID_val)

cb_list_Values.append([ForceLog,Force,vel,disp,ForceBool] $)$

return [cb_list_Values,presentImages]

else:

for $\mathrm{x}$ in range $(0$, sizeMove):

ForceBool $=$ False

if(presentImages $[\mathrm{x}][1][0]==$ Force and presentImages $[\mathrm{x}][1][1]==\mathrm{vel}$ and not(presentImages[x][1][3])):

isAVal $=$ True

presentImages[x][1][3]=True

presentImages[x][1].append(checkBox_ID_val)

ForceLog = wx.CheckBox(self.type_panel_f_d_6,checkBox_ID_val)

cb_list_Values.append([ForceLog,Force, vel,disp,ForceBool] $)$

elif(presentImages[x][1][0]==Force and not(presentImages[x][1][1]==vel)):

isAVal $=$ True

presentImages.append([[Force,vel,disp,False],[Force,vel,disp,True,checkBox_ID_val]])

ForceLog = wx.CheckBox(self.type_panel_f_d_6,checkBox_ID_val)

cb_list_Values.append([ForceLog,Force,vel,disp,ForceBool] $)$

elif(presentImages[x][1][0]==Force and presentImages[x][1][1]==vel and presentImages[x][1][3]):

isAVal $=$ True

presentImages[x][1] = [Force,vel,disp,True,checkBox_ID_val]

ForceLog = wx.CheckBox(self.type_panel_f_d_6,checkBox_ID_val)

cb_list_Values.append([ForceLog,Force,vel,disp,ForceBool])

if(not(isAVal)):

presentImages.append([[Force,vel,disp,False],[Force,vel,disp,True,checkBox_ID_val]])

ForceLog = wx.CheckBox(self.type_panel_f_d_6,checkBox_ID_val)

cb_list_Values.append([ForceLog,Force,vel,disp,ForceBool])

return [cb_list_Values,presentImages] 
def forceViewTableWrite (self,cb_list_Values,presentImages):

valueLength $=$ len(self.force_sizer_f_d_6.GetChildren())

if self.type_sizer_f_d_6.GetChildren():

for $\mathrm{x}$ in range $(0$,valueLength- 2$)$ :

self.force_sizer_f_d_6.Hide(valueLength-x-1)

self.force_sizer_f_d_6.Remove(valueLength-x-1)

self.force_panel_f_d_6.Layout()

self.disp_sizer_f_d_6.Hide(valueLength-x-1)

self.disp_sizer_f_d_6.Remove(valueLength-x-1)

self.disp_panel_f_d_6.Layout()

self.velocity_sizer_f_d_6.Hide(valueLength-x-1)

self.velocity_sizer_f_d_6.Remove(valueLength-x-1)

self.velocity_panel_f_d_6.Layout()

self.type_gridSizer_f_d_6.Destroy()

self.type_gridSizer_f_d_6 $=$ wx.GridSizer $(0,2,0,0)$

\#if self.type_gridSizer_f_d_6.GetChildren():

\# for $\mathrm{x}$ in range(0,len(self.type_gridSizer_f_d_6.GetChildren())):

$\#$ \#if $(x \% 2==0)$ :

\# self.type_gridSizer_f_d_6.Hide(x)

\# self.type_gridSizer_f_d_6.Remove(x)

\# self.type_gridSizer_f_d_6.Layout()

\# print len(self.type_gridSizer_f_d_6.GetChildren())

for $\mathrm{x}$ in range( 0 ,len(presentImages)):

if(presentImages[x][0][3]):

boxId $=$ presentImages $[\mathrm{x}][0][4]$

for $\mathrm{y}$ in range $(0$, len(cb_list_Values)):

if (boxId == cb_list_Values[y][0].GetId()):

forceBox $=$ cb_list_Values[y][0]

self.type_gridSizer_f_d_6.Add(forceBox,0,wx.ALL|wx.ALIGN_CENTER_HORIZONTAL, 5 )

else:

noText = wx.StaticText( self.type_panel_f_d_6, wx.ID_ANY, u"_", wx.DefaultPosition, wx.DefaultSize, wx.ALIGN_LEFT )

self.type_gridSizer_f_d 6.Add(noText,0,wx.ALL|wx.ALIGN_CENTER_HORIZONTAL, 5)

\#wx.EVT_CHECKBOX(self,checkBox_ID,self.dispBoxClick)

if(presentImages[x][1][3]):

boxId $=$ presentImages $[\mathrm{x}][1][4]$

for y in range(0,len(cb_list_Values)):

if(boxId == cb_list_Values[y][0].GetId()):

dispBox $=$ cb_list_Values[y][0]

self.type_gridSizer_f_d_6.Add(dispBox,0,wx.ALL|wx.ALIGN_CENTER_HORIZONTAL, 5 )

else:

noText = wx.StaticText( self.type_panel_f_d_6, wx.ID_ANY, u"_", wx.DefaultPosition, wx.DefaultSize, wx.ALIGN_LEFT )

self.type_gridSizer_f_d_6.Add(noText,wx.ID_ANY,wx.ALL|wx.ALIGN_CENTER_HORIZONTAL, 5)

self.type_gridSizer_f_d_6.Layout()

self.type_sizer_f_d_6.Addd(self.type_gridSizer_f_d_6)

\#self.type_sizer_f_d_6.Fit( self.type_panel_f_d_6 )

\#self.type_panel_f_d_6.Layout()

for $\mathrm{x}$ in range $(0$,len(presentImages)):

forceLog = wx.StaticText( self.force_panel_f_d_6, -1, str(presentImages[x][0][0]))

self.force_sizer_f_d_6.Add( forceLog, 0, wx.ALL|wx.ALIGN_CENTER_HORIZONTAL, 5 )

self.force_panel_f_d_6.Layout()

self.force_sizer_f_d_6.Fit( self.force_panel_f_d_6 )

dispLog = wx.StaticText $($ self.disp_panel_f_d_6, $-1, \operatorname{str}(\operatorname{presentImages[x][0][2]))}$

self.disp_sizer_f_d_6.Add( dispLog, 0, wx.ALL|wx.ALIGN_CENTER_HORIZONTAL, 5 )

self.disp_panel_f_d_6.Layout()

self.disp_sizer_f_d_6.Fit( self.disp_panel_f_d_6 )

velLog $=$ wx.StaticText $($ self.velocity_panel_f_d_6, $-1, \operatorname{str}($ presentImages[x][0][1]))

self.velocity_sizer_f_d_6.Add( velLog, 0, wx.ALL|wx.ALIGN_CENTER_HORIZONTAL, 5 )

self.velocity_panel_f_d_6.Layout()

self.velocity_sizer_f_d_6.Fit( self.velocity_panel_f_d_6 )

def $\log$ Write (self,type_indent,time,disp,force,vel,acc,hold):

global fileLoc

global save_log_dialogFileName

global yInt 
if (type_indent $==$ "Manual"):

FILE = open(fileLoc+'Il'+ save_log_dialogFileName+'.txt',"a")

FILE.write("time: " + str(time) + " , " + "vel: " +str(vel)+ " , " + "\{Manual MODE engaged $\} \backslash n ")$

elif(type_indent $==$ "Force"):

FILE = open(fileLoc+'ll'+ save_log_dialogFileName+'.txt',"a")

FILE.write("type: " + str(type_indent) +"force: " + force + ", time: " + str(time) + " , " + "disp: " + str(disp) + " , " + "vel: " + str(vel) + " , " + "acc: " + str(acc) +"ไn")

else:

FILE = open(fileLoc+'ll'+ save_log_dialogFileName+'.txt',"a")

FILE. write("Calibration Parameters: Voltage = " + "6.022*force + " + str(yInt)+"|n")

FILE.write("type: " + str(type_indent) + ", " +"force: " + force + ", time: " + str(time) + " , " + "disp: " + str(disp) + " , " + "vel: " + str(vel) + " , " + "acc: " + str(acc) +"'ln")

typeLog = wx.StaticText( self.type_panel, $-1, \operatorname{str}($ type_indent))

self.type_sizer.Add( typeLog, 0, wx.ALL|wx.ALIGN_RIGHT, 5 )

self.type_panel.Layout()

self.type_sizer.Fit( self.type_panel )

timeLog $=$ wx.StaticText (self.time_panel, $-1, \operatorname{str}($ time $))$

self.time_sizer.Add( timeLog, 0, wx.ALL|wx.ALIGN_RIGHT, 5 )

self.time_panel.Layout()

self.time_sizer.Fit( self.time_panel )

dispLog $=$ wx.StaticText( self.disp_panel, $-1, \operatorname{str}($ disp$)$ )

self.disp_sizer.Add( dispLog, 0, wx.ALL|wx.ALIGN_RIGHT, 5 )

self.disp_panel.Layout()

self.disp_sizer.Fit( self.disp_panel )

forceLog $=$ wx.StaticText( self.force_panel, $-1, \operatorname{str}($ force $)$ )

self.force_sizer.Add( forceLog, 0, wx.ALL|wx.ALIGN_RIGHT, 5 )

self.force_panel.Layout()

self.force_sizer.Fit( self.force_panel )

velLog $=$ wx.StaticText ( self.vel_panel, $-1, \operatorname{str}(v e l))$

self.vel_sizer.Add( velLog, 0, wx.ALL|wx.ALIGN_RIGHT, 5 )

self.vel_panel.Layout()

self.vel_sizer.Fit( self.vel_panel )

accLog $=$ wx.StaticText $($ self.acc_panel, $-1, \operatorname{str}(\operatorname{acc}))$

self.acc_sizer.Add( accLog, 0, wx.ALL|wx.ALIGN_RIGHT, 5 )

self.acc_panel.Layout()

self.acc_sizer.Fit( self.acc_panel )

holdLog $=$ wx.StaticText $($ self.hold_panel, $-1, \operatorname{str}($ hold $))$

self.hold_sizer.Add( holdLog, 0, wx.ALL|wx.ALIGN_RIGHT, 5 )

self.hold_panel.Layout()

self.hold_sizer.Fit( self.hold_panel )

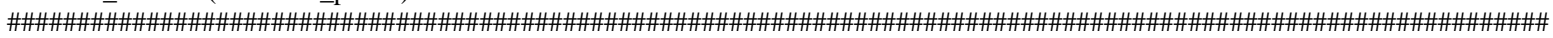

\#

\# Page one - Manual Mode Indentations

\#

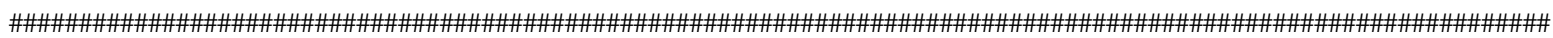

def Engage(self, event):

global manual_speed

global manMode

global GPIOLocation

global Manual thread

global Manual_buttonCol

global Alone

Alone $=$ False

self.resetEventTimer()

current_Time $=$ datetime.datetime.now ()

logTime_String = current_Time.strftime("\%H:\%M:\%S")

self.Status.SetLabel("Manual")

self.Status.SetForegroundColour((255,0,0))

self.sizer_panel.Layout()

\#statusEntry.SetValue("Manual Mode")

\#statusEntry.SetForegroundColour(wx.RED)

Manual_buttonCol = self.Engage_Button.GetBackgroundColour()

manual_speed = float(self.vel_appoach_1.GetValue())

self.logWrite ("Manual" ,logTime_String,,",",manual_speed,",")

manMode $=$ True

GPIOLocation = "GPIO2.ADC2"

self.Engage_Button.SetForegroundColour(wx.SystemSettings.GetColour(wx.SYS_COLOUR_BTNHIGHLIGHT ) ) 
self.Engage_Button.SetBackgroundColour(wx.SystemSettings.GetColour( wx.SYS_COLOUR_3DDKSHADOW ) ) self.Disengage_Button.SetForegroundColour(wx.SystemSettings.GetColour(wx.SYS_COLOUR_BTNTEXT) )

self.Disengage_Button.SetBackgroundColour(Manual_buttonCol )

self.indent_poc_1.Disable()

self.setPOC_button_1.Disable()

Manual_thread = ManualThread ()

def Disengage(self,event):

global manMode

global Manual_buttonCol

global potent_Mode

manMode $=$ False

time.sleep $(1)$

self.resetEventTimer()

self.Disengage_Button.SetForegroundColour( wx.SystemSettings.GetColour( wx.SYS_COLOUR_BTNHIGHLIGHT ) )

self.Disengage_Button.SetBackgroundColour( wx.SystemSettings.GetColour(wx.SYS_COLOUR_3DDKSHADOW ) )

self.Engage_Button.SetForegroundColour(wx.SystemSettings.GetColour(wx.SYS_COLOUR_BTNTEXT) )

self.Engage_Button.SetBackgroundColour(Manual_buttonCol )

self.indent_poc_1.Enable()

self.setPOC_button_1.Enable()

self.Status.SetLabel("Ready")

self.Status.SetForegroundColour((101,218,80))

self.sizer_panel.Layout()

potent_Mode $=$ loadCell ()

def UpdateSpeed(self,event):

global areStopped

global upSpeed

global downSpeed

areStopped $=$ True

if(areStopped):

upSpeed = (-float(self.vel_appoach_1.GetValue()))

downSpeed $=($ float $($ self.vel_appoach_1.GetValue ()$))$

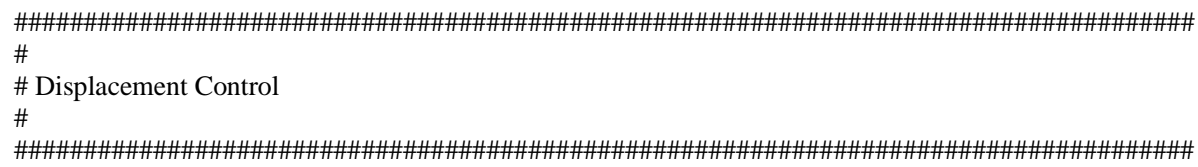

def displacementControlFunct(self,disp_Type,yInt):

global pointOfContact_Disp

global fileLoc

global save_log_dialogFileName

global servoCycle

global checkBox_ID

global checkBox_ID_f

global Alone

global potent_Mode

self.Status.SetForegroundColour((255,0,0))

self.Status.SetLabel("Busy")

self.sizer_panel.Layout()

dispFileName $="$

forceFileName $="$

type_disp $=$ "

Cycle $=$ False

Retract $=$ False

Extend $=$ False

Alone $=$ False

if $($ disp_Type $==$ ' 1 '):

displacement $=$ float(self.disp_spin_21.GetValue ()$)$

velocity $=$ float(self.vel_spin_21.GetValue())

acceleration = float(self.acc_spin_21.GetValue())

hold = float(self.hold_spin_21.GetValue())

if(self.cycle_21.GetValue()):

Cycle $=$ self.cycle_21.GetValue ()

type_disp = ' $\mathrm{C}$ '

Retract $=$ False

Extend $=$ False 
if(self.retract_21.GetValue()):

Retract $=$ self.retract_21.GetValue()

type_disp $=$ 'R'

Extend $=$ False

Cycle $=$ False

if(self.extend 21.GetValue()):

Extend $=$ self.extend_21.GetValue()

type_disp = 'E'

Cycle $=$ False

Retract $=$ False

elif $\left(\right.$ disp_Type $==$ ' $^{2}$ '):

displacement $=$ float(self.disp_spin_2.GetValue ()$)$

velocity $=$ float(self.vel_spin_2.GetValue())

acceleration $=$ float $\left(\right.$ self.acc $\left.\_s p i n \_2 . G e t V a l u e()\right)$

hold $=$ float(self.hold_spin_2.GetValue())

if(self.cycle_2.GetValue()):

Cycle $=$ self.cycle_2.GetValue ()

type_disp = 'C'

Retract $=$ False

Extend $=$ False

if(self.retract_2.GetValue()):

Retract $=$ self.retract_2.GetValue()

type_disp = 'R'

Extend $=$ False

Cycle $=$ False

if(self.extend_2.GetValue()):

Extend $=$ self.extend_2.GetValue ()

type_disp = 'E

Cycle $=$ False

Retract $=$ False

elif(disp_Type $==$ ' 3 '):

displacement $=$ float $($ self.disp_spin_3.GetValue ()$)$

velocity = float(self.vel_spin_3.GetValue())

acceleration $=$ float $($ self.acc_spin_3.GetValue())

hold = float(self.hold_spin_3.GetValue())

if(self.cycle_3.GetValue()):

Cycle $=$ self.cycle_3.GetValue ()

type_disp = ' $\mathrm{C}$ '

Retract $=$ False

Extend $=$ False

if(self.retract_3.GetValue()):

Retract $=$ self.retract_3.GetValue()

type_disp $=$ 'R'

Extend $=$ False

Cycle $=$ False

if(self.extend_3.GetValue()):

Extend $=$ self.extend_3.GetValue()

type_disp = 'E'

Cycle $=$ False

Retract $=$ False

elif(disp_Type $==$ 'D'):

displacement $=$ float $($ self.disp_spin_dp_5.GetValue ()$)$

velocity $=$ float $($ self.vel_spin_dp_5.GetValue ()$)$

acceleration $=$ float $($ self.acc_spin_dp_5.GetValue ()$)$

hold $=$ float(self.hold_spin_dp_5.GetValue ())

if(self.cycle_dp_5.GetValue()):

Cycle $=$ self.cycle_dp_5.GetValue ()

type_disp = ' $\mathrm{C}$

Retract $=$ False

Extend $=$ False

myIndenter.Record_DispControl(socketId,positioner,hold,servoCycle)

if(self.retract_dp_5.GetValue()):

Retract $=$ self.retract_dp_5.GetValue ()

type_disp = 'R'

Extend $=$ False

Cycle $=$ False

if(self.extend_dp_5.GetValue()):

Extend $=$ self.extend_dp_5.GetValue() 


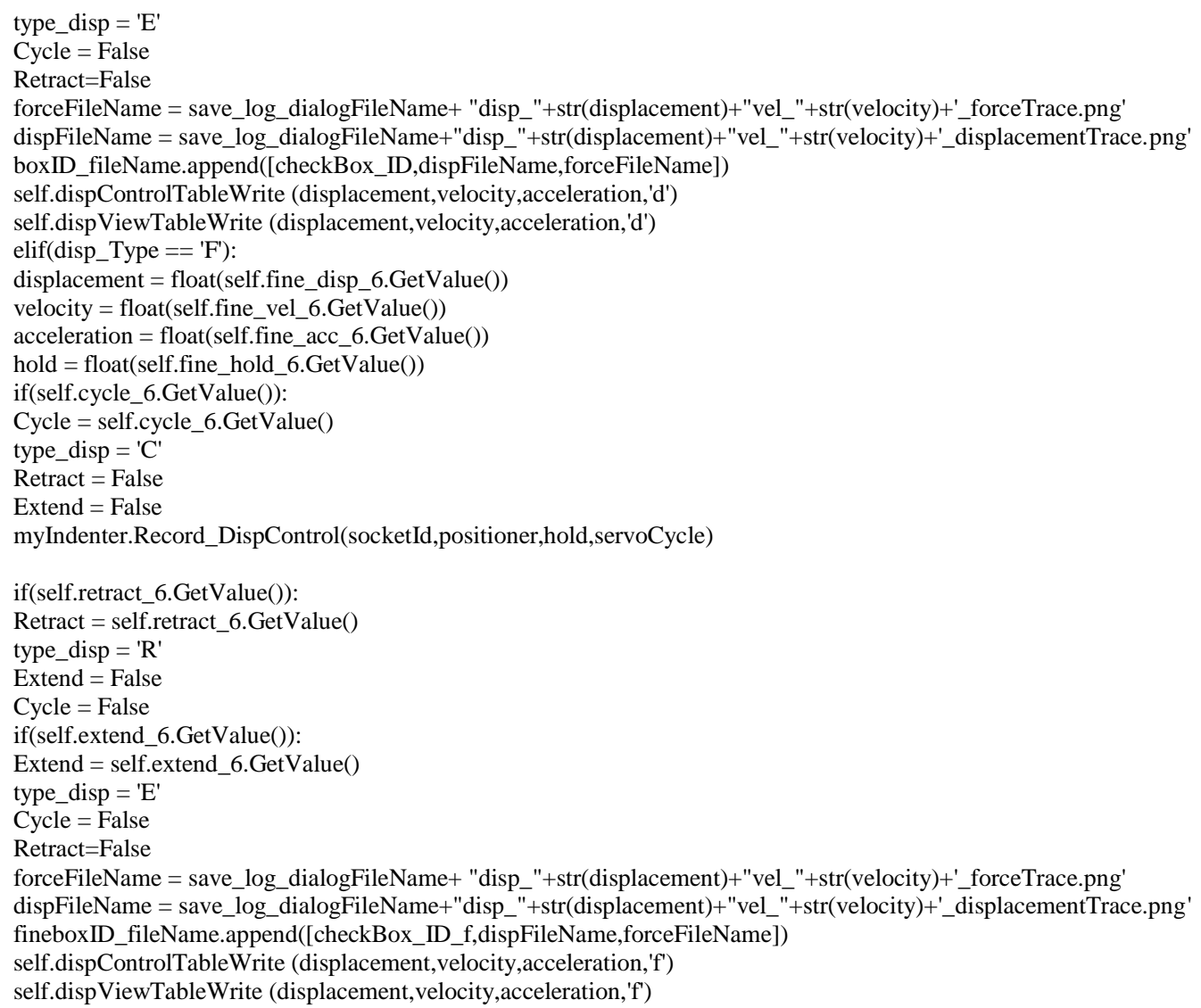

myIndenter.output(chosenForce,velocity,displacement,hold,pointOfContact_Disp,socketId,fileLoc,yInt,servoCycle,dispFileName,forceFileName, typeIndent)

current_Time $=$ datetime.datetime.now () 


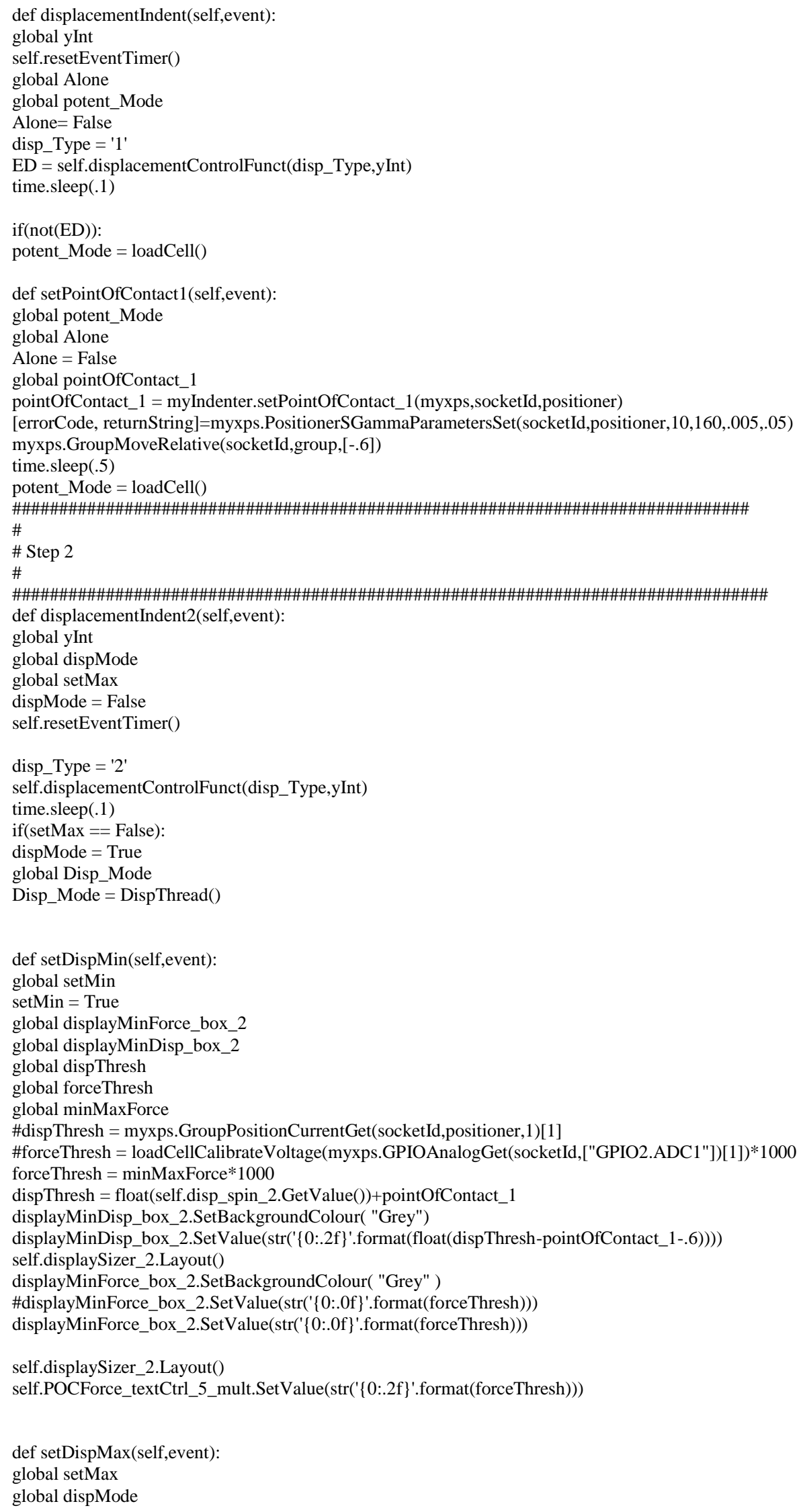




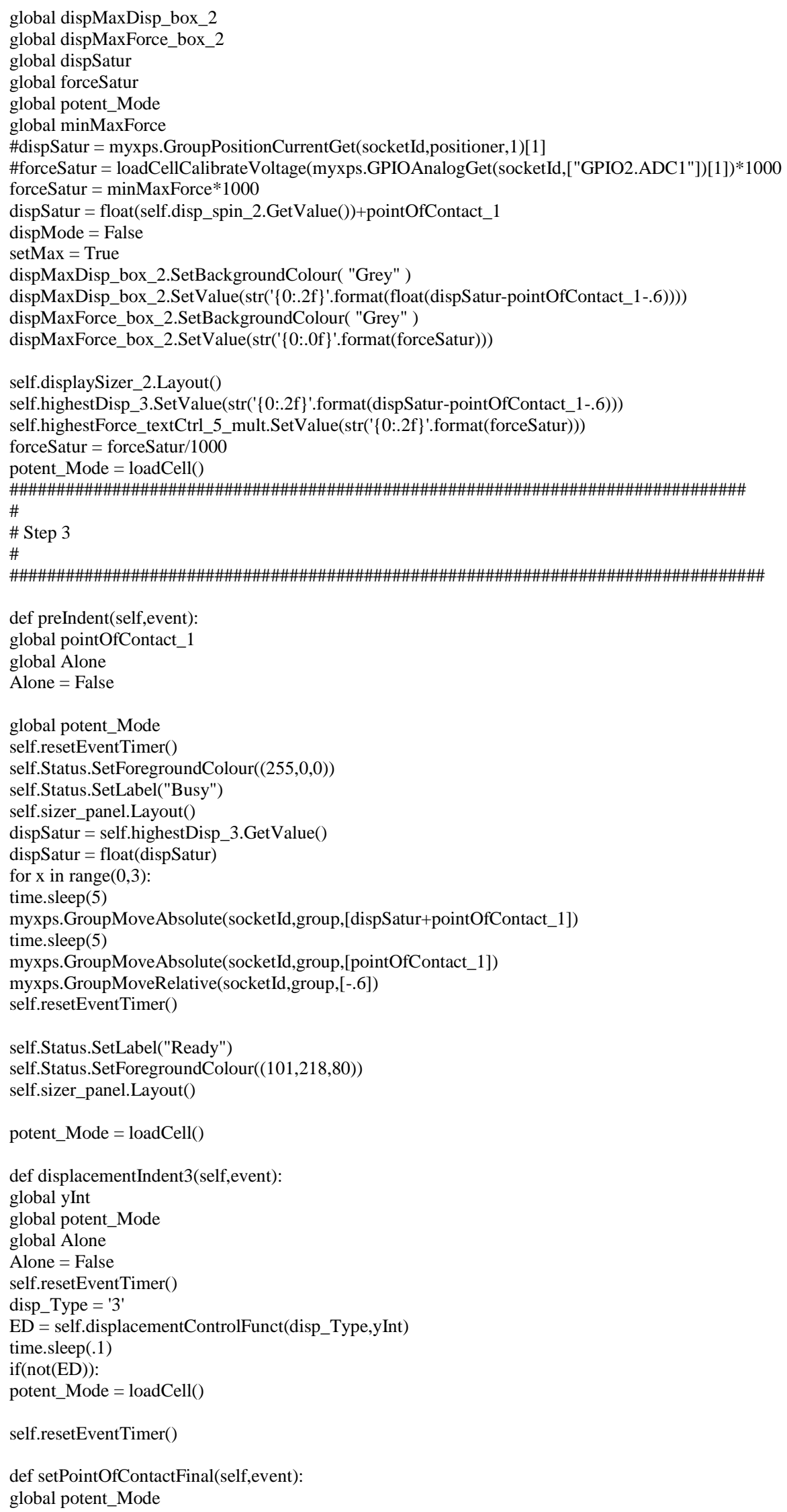




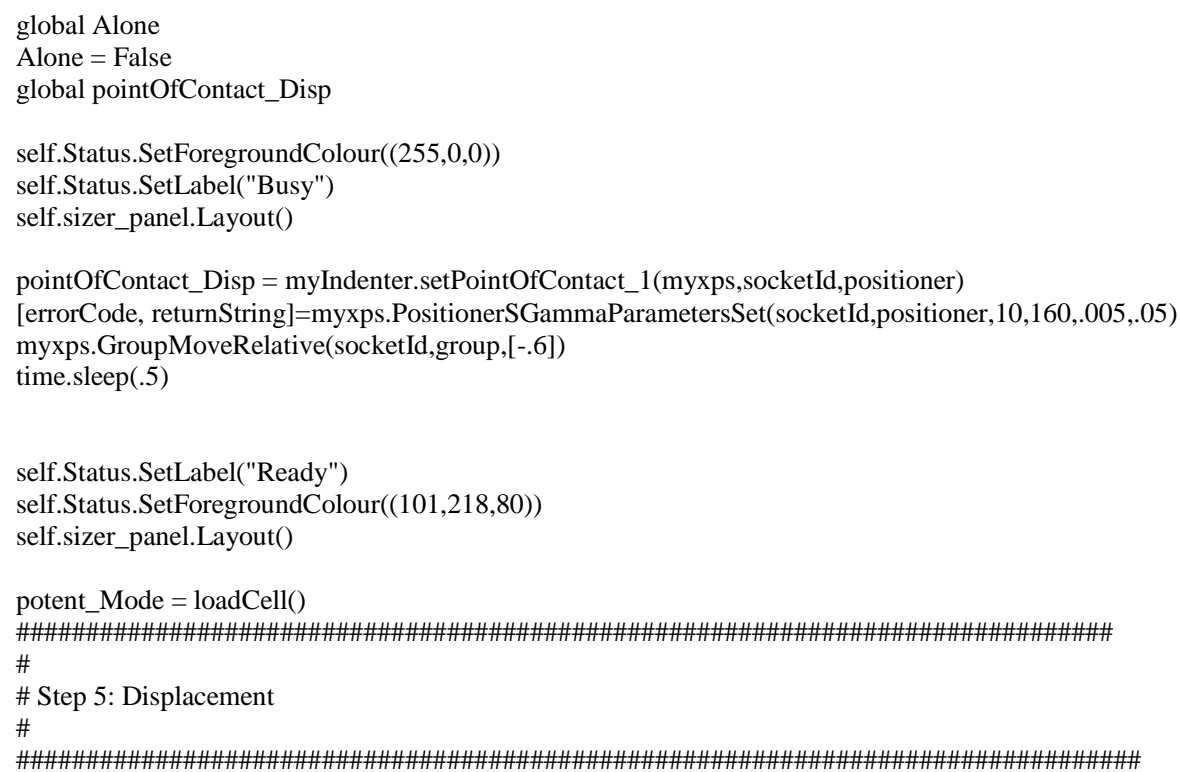

def displacementIndentExp(self,event):

global Alone

global yInt

global potent_Mode

Alone $=$ False

self.resetEventTimer()

disp_Type $=$ 'D'

$\mathrm{ED}=$ self.displacementControlFunct(disp_Type,yInt)

if(not(ED)):

potent_Mode $=$ loadCell ()

self.resetEventTimer()

def scale_bitmap(self,bitmap, width, height):

image $=$ wx.ImageFromBitmap $($ bitmap $)$

image $=$ image.Scale $($ width , height, wx.IMAGE_QUALITY_HIGH)

result $=\mathrm{wx}$.BitmapFromImage $($ image $)$

return result

def viewDispPlots(self,event):

global boxID_fileName

global cb_list

cb_Id = []

for $\mathrm{x}$ in range(0,len(cb_list)):

if(cb_list[x][0].GetValue()):

cb_Id.append(cb_list[x][0].GetId())

forcePlot $=$ []

dispPlot $=[]$

for $\mathrm{x}$ in range(0,len(boxID_fileName)):

for $y$ in range $(0$, len $(\mathrm{cb}$ Id $))$ :

if(boxID_fileName[x][0]==cb_Id[y]):

dispPlot.append(boxID_fileName[x][1])

forcePlot.append(boxID_fileName[x][2])

frame $=$ PicturePopUp()

global plotScroll

global plotSizer

for $\mathrm{i}$ in range(0,len(dispPlot)):

dispPlotImage $=$ wx.Image $($ fileLoc+'Il'+dispPlot[i], wx.BITMAP_TYPE_ANY).ConvertToBitmap ()

forcePlotImage $=$ wx.Image(fileLoc+' I'+forcePlot[i], wx.BITMAP_TYPE_ANY).ConvertToBitmap ()

disIndex $=$ dispPlot[i].find('disp_')

velIndex $=$ dispPlot[i].find('vel_')

$\operatorname{dispVal}=\operatorname{dispPlot}[\operatorname{disIndex}+5: \operatorname{disIndex}+8]$

velVal $=\operatorname{dispPlot}[$ velIndex+4:velIndex+7]

self.plotPanel $=$ wx.Panel $($ plotScroll, $\mathrm{i}+4000$, wx.DefaultPosition, wx.DefaultSize, wx.TAB_TRAVERSAL $)$

self.plotPanel.SetMinSize( wx.Size(500,-1 )) 
plotPanel_sizer_pop $=$ wx.FlexGridSizer $(2,1,0,0)$

plotPanel_sizer_pop.SetFlexibleDirection(wx.VERTICAL )

plotPanel_sizer_pop.SetNonFlexibleGrowMode( wx.FLEX_GROWMODE_SPECIFIED )

plotPanel_sizer_pop.SetMinSize( wx.Size(500,-1 ) )

self.textHeader_pop $=$ wx.StaticText( self.plotPanel, wx.ID_ANY, "Displacement Control: Displ. - "+ str(cb_list[i][1])+ " Velocity - " +

str(cb_list[i][2]) + " Accel.- " + str(cb_list[i][3]), wx.DefaultPosition, wx.DefaultSize, 0 )

self.textHeader_pop.Wrap( -1 )

self.textHeader_pop.SetFont( wx.Font( 14, 74, 90, 92, False, "Calibri" ) )

self.textHeader_pop.SetMinSize( wx.Size(500,-1 ))

plotPanel_sizer_pop.Add( self.textHeader_pop, 0, wx.ALL, 5 )

plots $=$ wx.GridSizer $(0,2,0,0)$

plots.SetMinSize( wx.Size (500,100) )

dispbitmap $=$ self.scale_bitmap $($ dispPlotImage, 200,100$)$

self.displaceImage $=$ wx.StaticBitmap(self.plotPanel, -1 , dispbitmap)

plots.Add( self.displaceImage, 0, wx.TOP|wx.BOTTOM|wx.ALIGN_CENTER, 5 )

forcebitmap $=$ self.scale_bitmap $($ forcePlotImage, 200,100$)$

self.forceImage $=w x$. StaticBitmap $($ self.plotPanel, -1 , forcebitmap)

plots.Add( self.forceImage, 0, wx.TOP|wx.BOTTOM|wx.ALIGN_CENTER, 5 )

plotPanel_sizer_pop.Add( plots, 1, wx.EXPAND, 5 )

self.plotPanel.SetSizer( plotPanel_sizer_pop )

self.plotPanel.Layout()

plotPanel_sizer_pop.Fit( self.plotPanel )

plotSizer.Add(self.plotPanel,0,wx.ALL|wx.ALIGN_CENTER_HORIZONTAL, 5 )

plotScroll.Layout()

plotSizer.Fit( plotScroll )

\#print dispPlot

frame. Show()

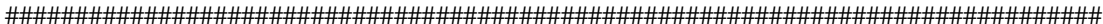

\#

\# Step 5: Force Control

\#

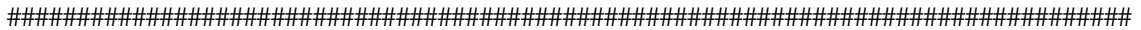

def Calibrate_Single(self,event):

global pointOfContact_Disp

global feedForward

global compForceCounter

global CompleteDisplacement

global CompleteForce

global forceSatur

global yInt

global trajectRoute

global Alone

global tauVal

POC $=$ pointOfContact_Disp

Alone $=$ False

self.Status.SetForegroundColour $((255,0,0))$

self.Status.SetLabel("Busy")

self.sizer_panel.Layout()

self.destroyTables()

self.resetEventTimer()

failure $=$ False

partition $=$ False

interval $="$

velIndex $=$ self.vel_choice_5.GetCurrentSelection()

vel $=$ self.vel_choice_5Choices[velIndex]

highForce $=$ self.highestForce_textCtrl_5.GetValue ()

saturation $="$

forceThresh $="$

vel $=$ float $($ vel $)$

highForce $=$ float $($ highForce $) / 1000$

CRCNSForce $=$

myIndenter.CalibrateCRCNSForce(interval,partition,vel,CompleteForce,CompleteDisplacement,forceThresh,pointOfContact_Disp,highForce,sat uration,compForceCounter,failure,feedForward,yInt,socketId, positioner,group,trajectRoute,True,forceSatur)

CompleteForce $=$ CRCNSForce $[0]$

compForceCounter $=$ CRCNSForce[1]

tauVal.append(CRCNSForce[2]) 


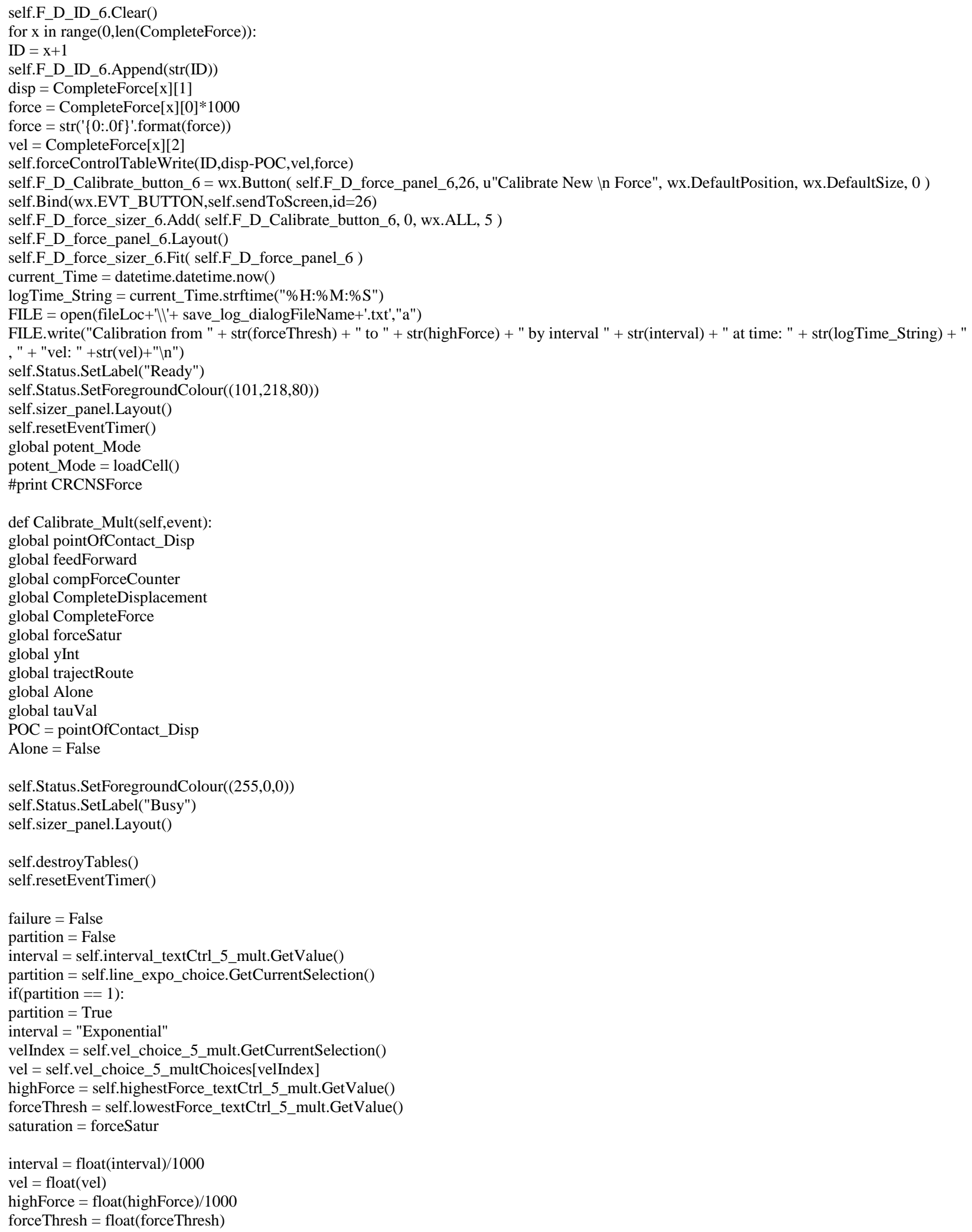




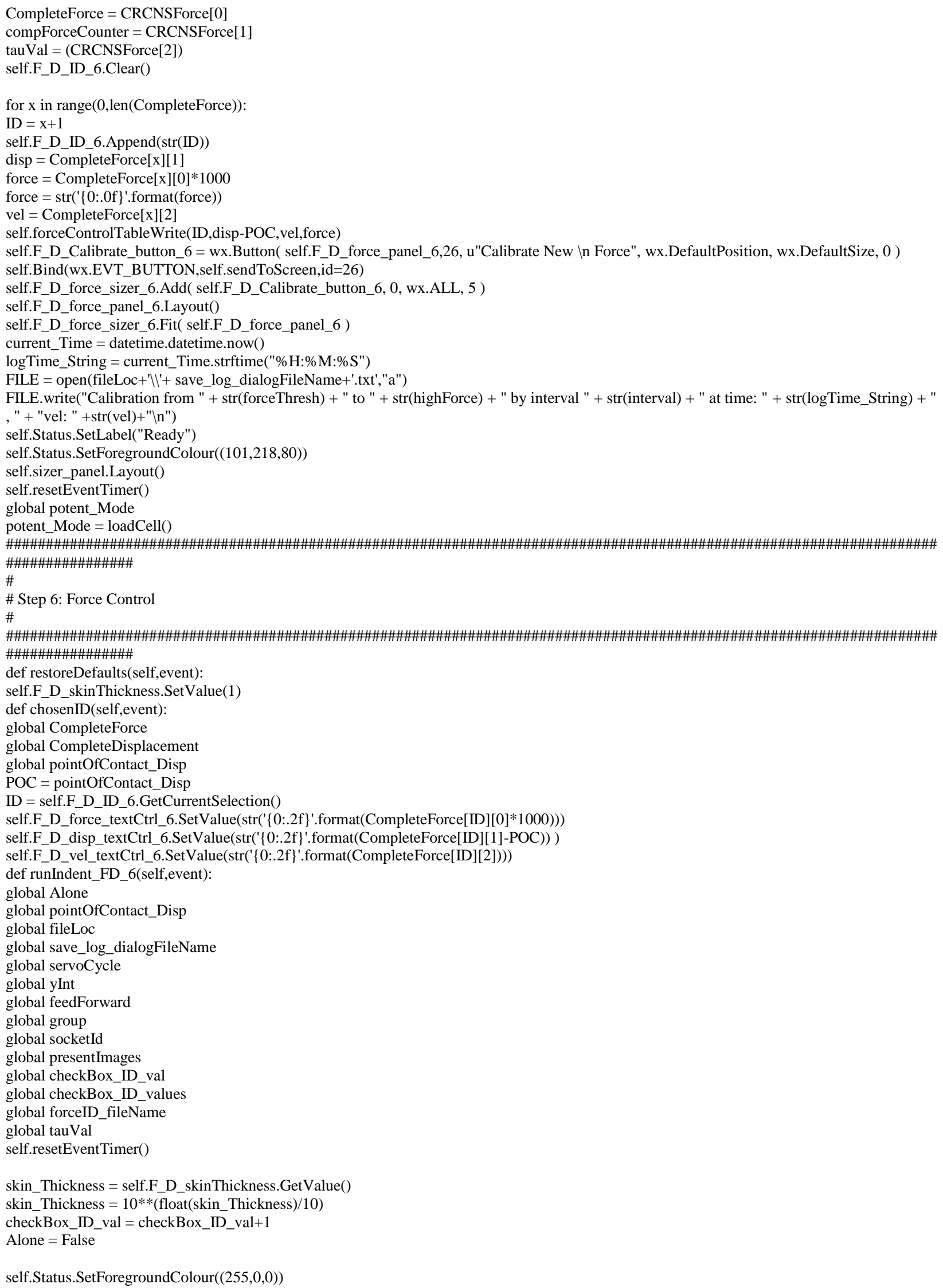


self.Status.SetLabel("Busy")

self.sizer_panel.Layout()

complete $=$ False

typeIndent $=$ "

ActInt $=$ self.F_D_ID_6.GetCurrentSelection()

Durstr $=$ self.F_D_holdTime_6.GetValue ()

velocity =float(self.F_D_vel_textCtrl_6.GetValue())

displacementExp $=$ CompleteForce[ActInt][1]

currentTau $=0$

totalTrajTime $=$ CompleteForce[ActInt][5]

for $\mathrm{x}$ in range(len(tauVal)):

if $(\operatorname{tauVal}[\mathrm{x}][0]==$ displacementExp and tauVal[x][1] ==velocity):

currentTau $=\operatorname{tauVal}[\mathrm{x}][2]$

Dur $=$ float(Durstr) -1.5

POC $=$ pointOfContact $\_$Disp

hold $=\operatorname{str}($ float $($ Dur $)+1.5)$

holdVal $=$ float(hold)

if(self.type_mec_Indent.GetCurrentSelection ()$==1)$ :

forceActVal $=$ int(ActInt)

AccMax $=160$

$\operatorname{expVol}=$ float $($ loadCellCalibrateForce $($ CompleteForce[ActInt][0]))

\#\#\# def RunIndentation(self,velocity, expDisp , Acceleration,

Duration,expVol,Force,idString,hairCycle,Diag,forceInd,POC,feedForward,positioner,socketId,group,fileLoc,yInt,servo,dispFileName,forceFile Name):

myIndenter.RunIndentation(velocity,displacementExp,AccMax,Dur,expVol,CompleteForce[ActInt][0],forceActVal,POC,CompleteForce[ActInt] [4],positioner,socketId,group,fileLoc,yInt,totalTrajTime,skin_Thickness)\#CompleteForce[ActInt][5],trajectRoute) current_Time $=$ datetime.datetime.now ()

$\log$ Time_String $=$ current_Time.strftime("\%H:\%M:\%S")

self.logWrite ("Force" ,logTime_String,displacementExp,str('\{0:.0f\}'.format(CompleteForce[ActInt][0]*1000)),velocity,",Durstr)

typeIndent $=$ ' $\mathrm{f}$ '

complete $=$ True

elif(self.type_mec_Indent.GetCurrentSelection ()$==0)$ :

DispActInt $=$ int(ActInt)

Dur $=$ Dur +1

myIndenter.dispForce(velocity,holdVal,DispActInt,CompleteForce[DispActInt][4],socketId,positioner,group,POC,displacementExp)

complete $=$ True

current_Time $=$ datetime.datetime.now ()

logTime_String = current_Time.strftime("\%H:\%M:\%S")

typeIndent $=$ 'd'

self.logWrite ("Disp. C" ,logTime_String,displacementExp,str('\{0:.0f \}'.format(CompleteForce[ActInt][0]*1000)),velocity,",Durstr)

else:

complete $=$ False

if(complete):

dispFileName $="$

forceFileName $="$

Force $="$

Forceprint $=$ CompleteForce $[$ ActInt $][0] * 1000$

if $($ len $($ presentImages $)==0)$ :

presentImages.append([[Forceprint,velocity,displacementExp-pointOfContact_Disp,False],[Forceprint,velocity,displacementExp-

pointOfContact_Disp,False]])

if (typeIndent == 'd'):

forceFileName = save_log_dialogFileName+ "disp_"+str(displacementExp-pointOfContact_Disp)+"vel_"+str(velocity)+'_forceTrace.png'

dispFileName = save_log_dialogFileName+"disp_"+str(displacementExp-pointOfContact_Disp)+"vel_"+str(velocity)+'_displacementTrace.png'

Force $=0$

fOd=False

checkBox_ID_values,presentImages = self.forceViewArrayWrite $($ Forceprint,displacementExp-

pointOfContact_Disp,velocity,fOd,checkBox_ID_val)

presentImages $=$ sorted $($ presentImages, $k$ ey=lambda $\mathrm{x}: \mathrm{x}[0])$

forceID_fileName.append([checkBox_ID_val,dispFileName,forceFileName])

\#self.forceViewTableWrite(checkBox_ID_values,presentImages) 
else:

forceFileName $=$ save_log_dialogFileName+

"Force_"+str('\{0:.0f $\}$ '.format(CompleteForce[ActInt][0]*1000))+"vel_"+str(velocity)+'_forceTrace.png' dispFileName $=$

save_log_dialogFileName+"Force_"+str('\{0:.0f\}'.format(CompleteForce[ActInt][0]*1000))+"vel_"+str(velocity)+'_displacementTrace.png'

Force $=$ CompleteForce $[$ ActInt $][0] * 1000$

fOd=True

\#if(5000+multInt $<=$ checkBox_ID_force):

checkBox_ID_values,presentImages = self.forceViewArrayWrite(Force,displacementExp-pointOfContact_Disp,velocity,f0d,checkBox_ID_val)

presentImages $=$ sorted $($ presentImages,key=lambda $\mathrm{x}: \mathrm{x}[0]$ )

\#self.forceViewTableWrite(checkBox_ID_values,presentImages)

forceID_fileName.append([checkBox_ID_val,dispFileName,forceFileName])

myIndenter.output(Force, velocity,displacementExp-

pointOfContact_Disp,hold,pointOfContact_Disp,socketId,fileLoc,yInt,50,dispFileName,forceFileName,typeIndent)

self.Status.SetLabel("Ready")

self.Status.SetForegroundColour((101,218,80))

self.sizer_panel.Layout()

self.resetEventTimer()

global potent_Mode

potent_Mode $=$ loadCell ()

def fine_Disp_6_Command(self,event):

global Alone

Alone $=$ False

global potent_Mode

global yInt

disp_Type $=$ ' $F$ '

$\mathrm{ED}=$ self.displacementControlFunct(disp_Type,yInt)

time.sleep(.1)

if(not(ED)):

potent_Mode $=$ loadCell ()

def view_ip(self,event):

global forceID_fileName

global checkBox_ID_values

global fineboxID_fileName

global cb_list_f

cb_Id_force $=$ []

cb_Id_fine $=[]$

for $x$ in range(0,len(checkBox_ID_values)):

if(checkBox_ID_values[x][0].GetValue()):

cb_Id_force.append(checkBox_ID_values[x][0].GetId())

for $\mathrm{x}$ in range(0,len(cb_list_f $)$ ):

if(cb_list_f $[\mathrm{x}][0]$.GetValue()):

cb_Id_fine.append(cb_list_f[x][0].GetId())

forcePlot $=[]$

dispPlot $=[]$

forcePlot_fine $=[]$

dispPlot_fine $=[]$

for $x$ in range(0,len(forceID_fileName)):

for $\mathrm{y}$ in range $\left(0, \mathrm{len}\left(\mathrm{cb} \_\mathrm{Id} \_\right.\right.$force $\left.)\right)$:

if(forceID_fileName[x][0]==cb_Id_force[y]):

dispPlot.append(forceID_fileName[x][1])

forcePlot.append(forceID_fileName[x][2])

for $\mathrm{x}$ in range( 0 ,len(fineboxID_fileName)):

for $y$ in range $(0$, len(cb_Id_fine $))$ :

if(fineboxID_fileName[x][0]==cb_Id_fine[y]):

forcePlot_fine.append(fineboxID_fileName[x][1])

dispPlot_fine.append(fineboxID_fileName[x][2])

frame $=$ PicturePopUp()

global plotScroll

global plotSizer

for $\mathrm{i}$ in range( 0 ,len(dispPlot)):

dispPlotImage $=$ wx.Image $($ fileLoc+' $\mid l '+\operatorname{dispPlot}[\mathrm{i}]$, wx.BITMAP_TYPE_ANY).ConvertToBitmap ()

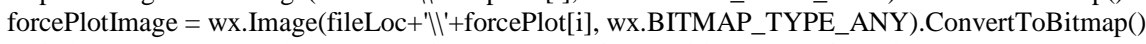

self.plotPanel $=$ wx.Panel $($ plotScroll, $i+4000, w x$. DefaultPosition, wx.DefaultSize, wx.TAB_TRAVERSAL $)$

self.plotPanel.SetMinSize( wx.Size(500,-1 ) )

plotPanel_sizer_pop $=$ wx.FlexGridSizer $(2,1,0,0)$

plotPanel_sizer_pop.SetFlexibleDirection(wx.VERTICAL ) 
plotPanel_sizer_pop.SetNonFlexibleGrowMode( wx.FLEX_GROWMODE_SPECIFIED )

plotPanel_sizer_pop.SetMinSize( wx.Size(500,-1 ) )

if(checkBox_ID_values[i][4]):

self.textHeader_pop $=$ wx.StaticText( self.plotPanel, wx.ID_ANY, "Force Control: Force - "+ str(checkBox_ID_values[i][1])+ " Displ.- " + str(checkBox_ID_values[i][3]) + " Vel.- " + str(checkBox_ID_values[i][2]), wx.DefaultPosition, wx.DefaultSize, 0 )

self.textHeader_pop.Wrap( -1 )

self.textHeader_pop.SetFont( wx.Font( 14, 74, 90, 92, False, "Calibri" ) )

self.textHeader_pop.SetMinSize( wx.Size $(500,-1)$ )

else:

self.textHeader_pop = wx.StaticText( self.plotPanel, wx.ID_ANY, "Displacement Control: Force - "+ str(checkBox_ID_values[i][1])+ " Displ.- "

+ $\operatorname{str}(\operatorname{checkBox}$ ID_values[i][3]) + " Vel.- " + str(checkBox_ID_values[i][2]), wx.DefaultPosition, wx.DefaultSize, 0 )

self.textHeader_pop.Wrap ( -1$)$

self.textHeader_pop.SetFont(wx.Font( 14, 74, 90, 92, False, "Calibri" ) )

self.textHeader_pop.SetMinSize( wx.Size $(500,-1)$ )

plotPanel_sizer_pop.Add( self.textHeader_pop, 0, wx.ALL, 5 )

plots $=$ wx.GridSizer $(0,2,0,0)$

plots.SetMinSize( wx.Size $(500,100)$ )

dispbitmap $=$ self.scale_bitmap (dispPlotImage, 200, 100)

self.displaceImage $=w x$. StaticBitmap (self.plotPanel, -1 , dispbitmap)

plots.Add( self.displaceImage, 0, wx.TOP|wx.BOTTOM|wx.ALIGN_CENTER, 5 )

forcebitmap $=$ self.scale_bitmap (forcePlotImage, 200, 100)

self.forceImage $=w x$. StaticBitmap $($ self.plotPanel, -1 , forcebitmap)

plots.Add( self.forceImage, 0, wx.TOP|wx.BOTTOM|wx.ALIGN_CENTER, 5 )

plotPanel_sizer_pop.Add( plots, 1, wx.EXPAND, 5 )

self.plotPanel.SetSizer(plotPanel_sizer_pop )

self.plotPanel.Layout()

plotPanel_sizer_pop.Fit( self.plotPanel )

plotSizer.Add(self.plotPanel,0,wx.ALL|wx.ALIGN_CENTER_HORIZONTAL, 5 )

plotScroll.Layout()

plotSizer.Fit( plotScroll )

\#print dispPlot

for $\mathrm{i}$ in range $(0$, len(dispPlot_fine $))$ :

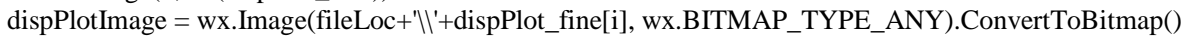

forcePlotImage $=$ wx.Image $($ fileLoc+'II'+forcePlot_fine[i], wx.BITMAP_TYPE_ANY).ConvertToBitmap ()

self.plotPanel $=$ wx.Panel $($ plotScroll, $i+4000$, wx.DefaultPosition, wx.DefaultSize, wx.TAB_TRAVERSAL $)$

self.plotPanel.SetMinSize( wx.Size (500,-1 ) )

plotPanel_sizer_pop $=$ wx.FlexGridSizer $(2,1,0,0)$

plotPanel_sizer_pop.SetFlexibleDirection(wx.VERTICAL)

plotPanel_sizer_pop.SetNonFlexibleGrowMode( wx.FLEX_GROWMODE_SPECIFIED )

plotPanel_sizer_pop.SetMinSize( wx.Size $(500,-1)$ )

self.textHeader_pop = wx.StaticText( self.plotPanel, wx.ID_ANY, "Displacement Control: Force - "+ str(cb_list_f[i][1])+ " Displ.- " + str(cb_list_f[i][2]) + " Velocity- " + str(cb_list_f[i][3]), wx.DefaultPosition, wx.DefaultSize, 0 )

self.textHeader_pop.Wrap ( -1$)$

self.textHeader_pop.SetFont( wx.Font( 14, 74, 90, 92, False, "Calibri" ) )

self.textHeader_pop.SetMinSize( wx.Size $(500,-1)$ )

plotPanel_sizer_pop.Add( self.textHeader_pop, 0 , wx.ALL, 5 )

plots $=$ wx.GridSizer $(0,2,0,0)$

plots.SetMinSize( wx.Size $(500,100)$ )

dispbitmap $=$ self.scale_bitmap $($ dispPlotImage, 200,100$)$

self.displaceImage $=w x$. StaticBitmap (self.plotPanel, -1 , dispbitmap)

plots.Add( self.displaceImage, 0, wx.TOP|wx.BOTTOM|wx.ALIGN_CENTER, 5 )

forcebitmap $=$ self.scale_bitmap $($ forcePlotImage, 200,100$)$

self.forceImage $=w x$. StaticBitmap (self.plotPanel, -1 , forcebitmap)

plots.Add( self.forceImage, 0, wx.TOP|wx.BOTTOM|wx.ALIGN_CENTER, 5 )

plotPanel_sizer_pop.Add( plots, 1, wx.EXPAND, 5 )

self.plotPanel.SetSizer(plotPanel_sizer_pop )

self.plotPanel.Layout()

plotPanel_sizer_pop.Fit( self.plotPanel )

plotSizer.Add(self.plotPanel,0,wx.ALL|wx.ALIGN_CENTER_HORIZONTAL, 5 )

plotScroll.Layout()

plotSizer.Fit( plotScroll )

frame. Show() 


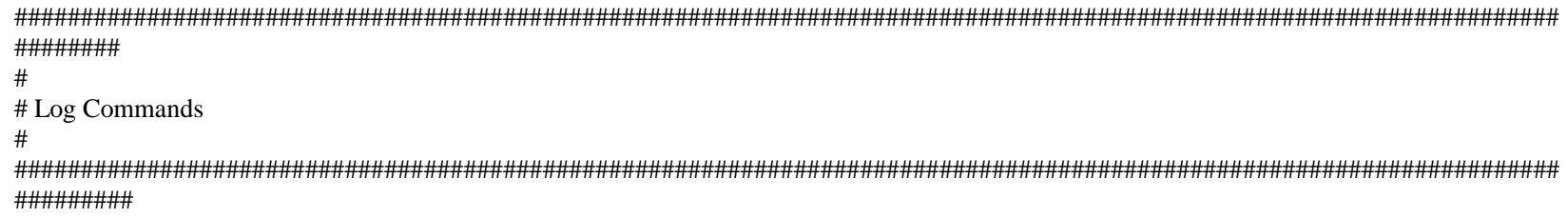




\section{Appendix D- Python code for the control algorithms}

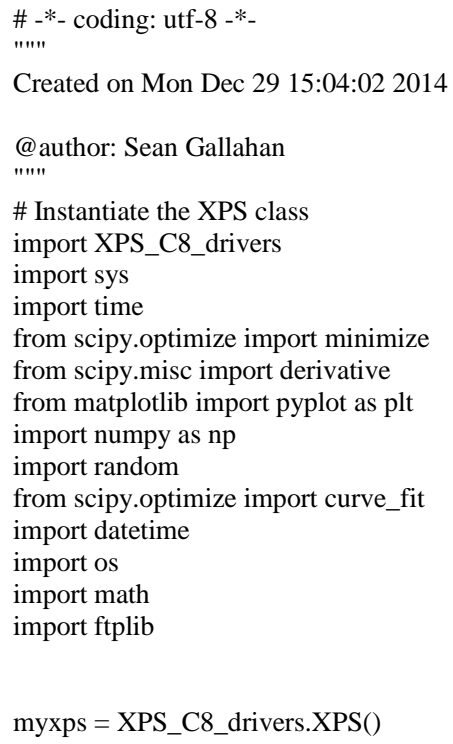




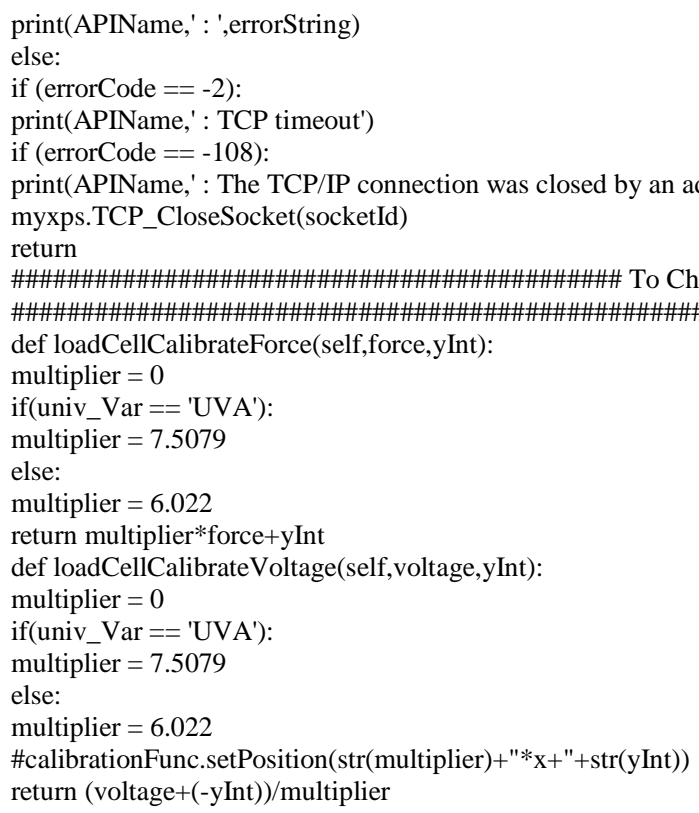

\#turns of group, restarts group, and homes group. Must be done every time the progrma is activated def greeting(self,socketId, group):

[errorCode, returnString] $=$ myxps. GroupKill(socketId, group)

if (errorCode $!=0$ ):

self.displayErrorAndClose (socketId, errorCode, 'GroupKill')

sys.exit()

[errorCode, returnString] $=$ myxps. GroupInitialize (socketId, group)

if (errorCode $!=0$ ):

self.displayErrorAndClose (socketId, errorCode, 'GroupInitialize')

sys.exit()

[errorCode, returnString $]=$ myxps .GroupHomeSearch $($ socketId, group)

if (errorCode != 0):

self.displayErrorAndClose (socketId, errorCode, 'GroupHomeSearch')

sys.exit()

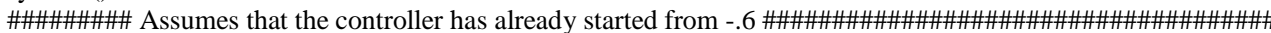

def fineDisplacementControl(self,myxps,socketId,positioner,group, displacement, velocity, acceleration, hold_duration, cycle, retract, extend,yInt):

[errorCode, returnString]=myxps.PositionerSGammaParametersSet(socketId,positioner,velocity,acceleration,.005,.05)

peakVolt $=0$

voltage $=$ myxps.GPIOAnalogGet $($ socketId,["GPIO2.ADC1"])[1]

if (cycle):

[errorCode, returnString] $=$ myxps.GroupMoveRelative (socketId, group, $[$ displacement] $)$

peakVolt $=$ myxps.GPIOAnalogGet(socketId,["GPIO2.ADC1"])[1]

\#if(self.loadCellCalibrateVoltage(voltage,yInt)>2):

\# myxps.GroupMoveRelative(socketId,group,[-displacement])

time.sleep(hold_duration)

[errorCode, returnString] $=$ myxps GroupMoveRelative $($ socketId, group,$[-($ displacement $)]$ )

elif (retract):

[errorCode, returnString] $=$ myxps.GroupMoveRelative $($ socketId, group, $[$-displacement] $)$

if(self.loadCellCalibrateVoltage(voltage,yInt)>2):

myxps.GroupMoveRelative(socketId,group,[-displacement])

else:

[errorCode, returnString] $=$ myxps. GroupMoveRelative (socketId, group, $[$ displacement] $)$

voltage $=$ myxps.GPIOAnalogGet(socketId,["GPIO2.ADC1"])[1]

if((self.loadCellCalibrateVoltage(voltage,yInt)>2)):

myxps.GroupMoveRelative(socketId,group,[-displacement])

return peakVolt

def setPointOfContact_1(self,myxps,socketId,positioner):

return myxps.GroupPositionCurrentGet(socketId,positioner,1)[1]

def Record_DispControl(self,socketId,positioner,holdTime,servoCycle):

if (univ_Var== 'COL'): 
servo $=.000125$

else:

servo $=.0001$

numbPoints $=($ holdTime +2$) /\left(\right.$ servo* ${ }^{*}$ servoCycle $)$

numbPoints $=$ int (numbPoints)

[errorCode,returnString]=myxps.GatheringReset(socketId)

if (errorCode != 0):

self.displayErrorAndClose (socketId, errorCode, 'GatheringReset')

sys.exit()

[errorCode, returnString]=myxps.GatheringConfigurationSet(socketId,[positioner+".CurrentPosition","GPIO2.ADC1"])

if (errorCode !=0):

self.displayErrorAndClose (socketId, errorCode, 'GatheringConfigurationSet')

sys.exit()

[errorCode, returnString]=myxps.EventExtendedConfigurationTriggerSet(socketId,[positioner+".SGamma.MotionStart"],["0"],["0"],["0"],["0"])

if (errorCode != 0):

self.displayErrorAndClose (socketId, errorCode, 'EventExtendedConfigurationTriggerSet')

sys.exit()

[errorCode,

returnString]=myxps.EventExtendedConfigurationActionSet(socketId,["GatheringRun"],[str(numbPoints)],[str(servoCycle)],["0"],["0"])

if (errorCode != 0):

self.displayErrorAndClose (socketId, errorCode, 'EventExtendedConfigurationActionSet')

sys.exit()

[errorCode, returnString]=myxps.EventExtendedStart(socketId)

if (errorCode $!=0$ ):

self.displayErrorAndClose (socketId, errorCode, 'Event Extended Start')

sys.exit()

def endRecord_DispControl(self,socketId,positioner,holdTime):

[errorCode, returnString] $=$ myxps. GatheringStopAndSave(socketId)

if (errorCode $!=0$ ):

self.displayErrorAndClose (socketId, errorCode, 'GatheringStopandSave')

sys.exit()

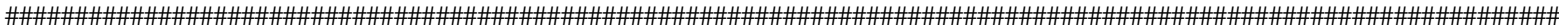

\#

\# Graphical Output

\#

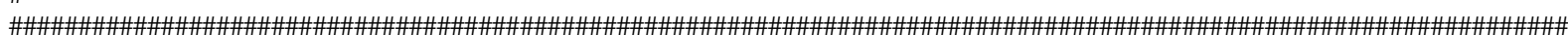

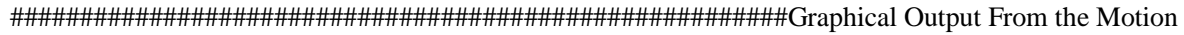

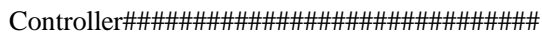

def output(self,chosenForce,vel,disp,hold,POC,socketId,fileLoc,yInt,servo,dispFileName,forceFileName,typeIndent):

global univ_Var

CurrentNumber $=$ myxps.GatheringCurrentNumberGet(socketId) $[1]$

iterations $=$ CurrentNumber $/ 500$

LeftOver $=$ CurrentNumber $\% 500$

Code $=[]$

$\mathrm{C}=[]$

starting $=0$

count $=0$

\#Iterates over the 500 points that the program can read at a maximum time

endCount $=500$

for $\mathrm{y}$ in range(iterations +1$)$ :

count $=$ count +1

if $(\mathrm{y}==$ (iterations) and (LeftOver $!=0))$ :

$\mathrm{C}=[$ errorCode, returnString $]=$ myxps.GatheringDataMultipleLinesGet(socketId,starting,LeftOver)

if (errorCode !=0):

self.displayErrorAndClose (socketId, errorCode, 'GatheringDataMultipleLinesGet')

sys.exit()

C.remove(0)

Code $[0]=$ Code $[0]+C[0]$

elif(y<iterations):

if $(\mathrm{y}==0)$ :

$\mathrm{C}=[$ errorCode, returnString $]=$ myxps.GatheringDataMultipleLinesGet(socketId,starting,endCount)

if (errorCode $!=0$ ):

self.displayErrorAndClose (socketId, errorCode, 'GatheringDataMultipleLinesGet')

sys.exit()

C.remove (0)

Code.append(C[0]) 
starting $=$ endCount + starting

else:

$\mathrm{C}=[$ errorCode, returnString $]=$ myxps.GatheringDataMultipleLinesGet(socketId,starting,endCount)

if (errorCode !=0):

self.displayErrorAndClose (socketId, errorCode, 'GatheringDataMultipleLinesGet')

sys.exit()

C.remove(0)

Code $[0]=\operatorname{Code}[0]+\mathrm{C}[0]$

starting $=$ endCount + starting

\#Creates arrays that will moderate over gathered data

$\mathrm{x}=$ Code[0].count(';')

displacement $=[\text { None }]^{*} \mathrm{x}$

Force $=[\text { None }]^{*} \mathrm{x}$

Forcestr $=[\text { None }]^{*} \mathrm{x}$

displacementstr $=[\text { None }]^{*} \mathrm{x}$

dis $=$ Code[0].index (';')

volt $=-1$

time $=[]$

timestr $=[]$

\#aboveRange =[]

\#belowRange=[]

multiplier $=0$

if(univ_Var == 'UVA'):

multiplier $=.0001$

else:

multiplier $=.000125$

for $\mathrm{m}$ in range(x):

$\mathrm{z}=\mathrm{m}^{*}$ multiplier*servo

time.append $(\mathrm{z})$

timestr.append $(\operatorname{str}(\mathrm{z}))$

FILE $=$ open(fileLoc+ 'force ' + str(chosenForce)+ ' vel '+str('\{0:.0f $\}$ '.format(vel))+'disp '+str('\{0:.0f $\}$ '.format(disp))+'TimeEntry'+'.txt',"a" $)$

FILE.write(timestr[m]+'ไn')

\#aboveRange.append(chosenForce*1.01)

\#belowRange.append(chosenForce*.99)

if $(m==x-1)$ :

displacement $[\mathrm{m}]=$ float $($ Code $[0][$ volt $+1:$ dis $])-(P O C)$

displacementstr[m] $=\operatorname{str}($ float $(\operatorname{Code}[0][$ volt $+1: \operatorname{dis}])-($ POC $))$

FILE = open(fileLoc+ 'force ' + str(chosenForce)+ ' vel '+str('\{0:.0f $\}$ '.format(vel))+'disp '+str('\{0:.0f $\}$ '.format(disp))+'DispEntry'+'.txt',"a")

FILE.write(displacementstr[m]+' $\mid n ')$

volt=Code[0].index('In',volt+1,len(Code[0]))

Force $[\mathrm{m}]=$ self.loadCellCalibrateVoltage $(($ float $($ Code[0][dis+1:volt $])), y I n t)$

Forcestr[m]=str(self.loadCellCalibrateVoltage $(($ float $($ Code[0][dis+1:volt] $)), y$ Int $))$

FILE $=$ open(fileLoc+ 'force ' + str(chosenForce)+ ' vel '+str(' $\{0: .0 f\}$ '.format(vel))+'disp '+str('\{0:..0f $\}$ '.format(disp))+'ForceEntry'+'.txt',"a")

FILE.write(Forcestr[m]+'ไn')

else:

displacement $[\mathrm{m}]=$ float $($ Code $[0][$ volt +1 :dis $])-(P O C)$

displacementstr[m] = str(float $($ Code $[0][$ volt+1:dis] $)-($ POC $))$

FILE $=$ open(fileLoc+'force ' + str(chosenForce)+ ' vel '+str('\{0:.0f $\}$ '.format(vel))+'disp '+str('\{0:.0f $\}$ '.format(disp))+'DispEntry'+'.txt',"a")

FILE.write(displacementstr[m]+' $\backslash \mathrm{n}$ ')

volt=Code[0].index('In',volt+1,len $(\operatorname{Code}[0]))$

Force $[\mathrm{m}]=$ self.loadCellCalibrateVoltage $(($ float $($ Code $[0][$ dis $+1:$ volt $])), y I n t)$

Forcestr[m] $=\operatorname{str}($ self.loadCellCalibrateVoltage ((float(Code[0][dis+1:volt] $)), y$ Int $))$

FILE = open(fileLoc+ 'force ' + str(chosenForce)+ ' vel '+str('\{0:.0f $\}$ '.format(vel))+'disp '+str('\{0:.0f $\}$ '.format(disp))+'ForceEntry'+'.txt',"a")

FILE.write(Forcestr[m]+'ไn')

dis $=$ Code[0].index(';',dis+1)

\#global trajectTimeAll

$\#$ trajectTime $=[]$

\#minDiff $=1$

\#closestVal $=0$

\#for $\mathrm{x}$ in range(0,len(trajectTimeAll)):

\#print abs(chosenForce/1000 - trajectTimeAll[x][0])

\# if(abs(chosenForce/1000 - trajectTimeAll[x][0])<minDiff):

\# minDiff $=$ abs $($ chosenForce/1000 - trajectTimeAll $[\mathrm{x}][0])$

$\#$ closestVal $=x$

\#trajectTime $=$ trajectTimeAll[closestVal][1] 
\#for $\mathrm{x}$ in range( 0 ,len(trajectTime)):

\# trajectTime $[\mathrm{x}]=$ self.myround(trajectTime $[\mathrm{x}])$

\# trajectTime $[\mathrm{x}]=\operatorname{str}('\{0: .4 \mathrm{f}\}$ '.format $($ trajectTime $[\mathrm{x}]))$

\# trajectTime $[\mathrm{x}]=$ float(trajectTime $[\mathrm{x}]$ )

\#trajectTimeVal = []

\#trajectForceVal = []

\#for $\mathrm{x}$ in range(0,len(trajectTime)):

\# trajectTimeVal.append(float(trajectTime[x]))

\# trajectForceVal.append(Force[int(float(trajectTimeVal[x])/.001)])

$\# \mathrm{xAxis}=$ hold +2

plt.figure $($ figsize $=(4.5,2.0))$

plt.xlabel("Time (s)")

if $($ typeIndent $==$ 'd'):

plt.title("Displacement: " + str(disp) + " Velocity: " + str(vel))

else:

plt.title("Displacement vs. Time")

plt.ylabel("Displacement (mm)")

plt.ylim(0,disp*1.2)

plt.plot(time,displacement,'b')

plt.savefig(fileLoc+'II'+dispFileName)

plt.figure $($ figsize $=(4.5,2.0))$

if $($ typeIndent $==$ ' $d$ '):

plt.title("Force vs. Time")

else:

plt.title("Force: " + str(chosenForce) + " vel: " + str(vel))

plt.xlabel("Time (s)")

plt.ylabel("Force $(\mathrm{mN}) ")$

plt.plot(time,Force,'b')\#trajectTimeVal,trajectForceVal,'g^')

plt.plot()

plt.savefig(fileLoc+'II'+forceFileName)

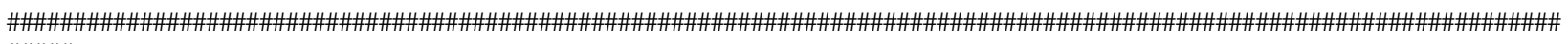
\#\#\#\#\#

\#

\# Force Control Calibration

\#

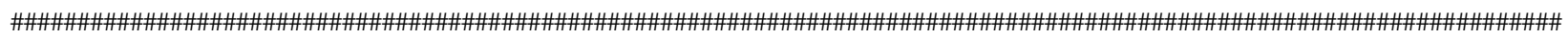
\#\#\#\#\#

def identifyLocation(self,maxForce, socketId,group,yInt,POC,positioner,vel):

[errorCode,returnString]=myxps.GatheringReset(socketId)

if(errorCode !=0):

self.displayErrorAndClose (socketId,errorCode,'GatheringReset')

sys.exit()

[errorCode, returnString]=myxps.GatheringConfigurationSet(socketId,[positioner+".CurrentPosition","GPIO2.ADC1"])

if (errorCode $!=0$ ):

self.displayErrorAndClose (socketId, errorCode, 'GatheringConfigurationSet')

sys.exit()

[errorCode, returnString]=myxps.EventExtendedConfigurationTriggerSet(socketId,[positioner+".Jog.MotionStart"],["0"],["0"],["0"],["0"])

if (errorCode !=0):

self.displayErrorAndClose (socketId, errorCode, 'EventExtendedConfigurationTriggerSet')

sys.exit()

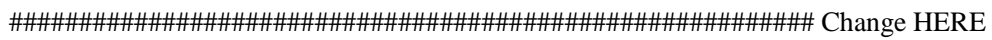

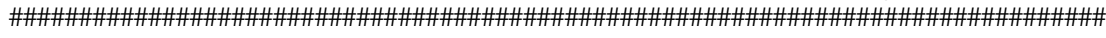

[errorCode, returnString]=myxps.EventExtendedConfigurationActionSet(socketId,["GatheringRun"],["25000"],["5"],["0"],["0"])

if (errorCode $!=0$ ):

self.displayErrorAndClose (socketId, errorCode, 'EventExtendedConfigurationActionSet')

sys.exit()

[errorCode, returnString]=myxps.EventExtendedStart(socketId)

if (errorCode != 0 ):

self.displayErrorAndClose (socketId, errorCode, 'Event Extended Start')

sys.exit() 
[errorCode,returnString]=myxps.GroupJogModeEnable(socketId,group)

if(errorCode $!=0$ ):

self.displayErrorAndClose (socketId, errorCode, 'GroupJogModeEnable')

sys.exit()

[errorCode, returnString] = myxps.GroupJogParametersSet(socketId, group,[vel],[80])

if (errorCode $!=0$ ):

self.displayErrorAndClose (socketId, errorCode, 'GroupJogParametersSet')

sys.exit()

voltage $=$ myxps.GPIOAnalogGet(socketId,["GPIO2.ADC1"])

expectedVolt $=$ self.loadCellCalibrateForce $(\operatorname{maxForce}, \mathrm{yInt}) * 1.01$

while (voltage $[1]<=$ expectedVolt):

if(self.loadCellCalibrateVoltage(voltage[1],yInt)>1.5):

myxps.GroupMoveAbsolute(socketId, group, [POC-.6])

voltage $=$ myxps.GPIOAnalogGet $($ socketId,["GPIO2.ADC1"])

[errorCode,returnString]=myxps.GroupMoveAbort(socketId,group)

[errorCode,returnString] $=$ myxps.GatheringStopAndSave $($ socketId $)$

if(errorCode! $=0$ ):

self.displayErrorAndClose(socketId,errorCode,'GatheringStopandSave')

sys.exit()

[errorCode, returnString] $=$ myxps .GroupMoveAbsolute $($ socketId, group, [POC-.6])

linOut $=$ self.linearOutput $(\mathrm{POC}$, socketId,yInt $)$

return linOut

\#Calibrate the Indenter for CRCNS Force (Multiple Displacements)

def linearOutput(self,POC,socketId,yInt):

CurrentNumber $=$ myxps.GatheringCurrentNumberGet(socketId) $[1]$

iterations $1=$ CurrentNumber $/ 500$

LeftOver $=$ CurrentNumber $\% 500$

Code $=[]$

$\mathrm{C}=[]$

starting $1=0$

count $1=0$

\#Iterates over the 500 points that the program can read at a maximum time

endCount $=500$

for $\mathrm{y}$ in range(iterations $1+1)$ :

count $1=$ count $1+1$

if $(\mathrm{y}==$ (iterations 1$)$ and (LeftOver $!=0)$ ):

$\mathrm{C}=$ [errorCode, returnString $]=$ myxps.GatheringDataMultipleLinesGet(socketId,starting1,LeftOver)

if (errorCode != 0):

self.displayErrorAndClose (socketId, errorCode, 'GatheringDataMultipleLinesGet')

sys.exit()

C.remove $(0)$

Code $[0]=\operatorname{Code}[0]+\mathrm{C}[0]$

elif(y<iterations1):

if $(\mathrm{y}==0)$ :

$\mathrm{C}=[$ errorCode, returnString $]=$ myxps.GatheringDataMultipleLinesGet(socketId,starting1,endCount)

if (errorCode $!=0$ ):

self.displayErrorAndClose (socketId, errorCode, 'GatheringDataMultipleLinesGet')

sys.exit()

C.remove $(0)$

Code.append $(\mathrm{C}[0])$

starting $1=$ endCount + starting 1

else:

$\mathrm{C}=[$ errorCode, returnString $]=$ myxps.GatheringDataMultipleLinesGet(socketId,starting1,endCount)

if (errorCode $!=0$ ):

self.displayErrorAndClose (socketId, errorCode, 'GatheringDataMultipleLinesGet')

sys.exit()

C.remove (0)

Code $[0]=\operatorname{Code}[0]+\mathrm{C}[0]$

starting 1 = endCount + starting 1

\#Creates arrays that will moderate over gathered data

$\mathrm{x}=$ Code[0].count (';')

Forcestr $=[\text { None }]^{*} \mathrm{x}$ 
displacementstr $=[$ None $] * \mathrm{x}$

identifyDisp $=[\text { None }]^{*} \mathrm{x}$

identifyForce $=[\text { None }]^{*} \mathrm{x}$

dis $=$ Code[0].index $(' ; ')$

volt $=-1$

time $=[]$

timestr=[]

for $\mathrm{m}$ in range(x):

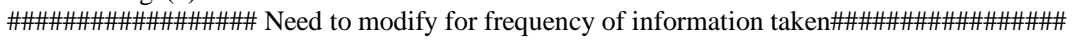

$\mathrm{z}=\mathrm{m}^{*} .0005$

time.append(z)

timestr.append( $\operatorname{str}(\mathrm{z}))$

if $(m==x-1)$ :

identifyDisp[m] = float $($ Code $[0][$ volt+1:dis $])-($ POC)

displacementstr $[\mathrm{m}]=\operatorname{str}($ float $($ Code $[0][$ volt $+1:$ dis $])-(P O C))$

volt=Code[0].index('In',volt+1,len(Code[0]))

identifyForce[m] = self.loadCellCalibrateVoltage $(($ float(Code[0][dis+1:volt])),yInt $)$

Forcestr[m]=str(self.loadCellCalibrateVoltage((float(Code[0][dis+1:volt])),yInt))+","

else:

identifyDisp $[\mathrm{m}]=$ float $($ Code $[0][$ volt+1:dis $])-($ POC $)$

displacementstr $[\mathrm{m}]=\operatorname{str}($ float $($ Code $[0][$ volt $+1: \operatorname{dis}])-(\mathrm{POC}))$

volt=Code[0].index('In',volt+1,len(Code[0]))

identifyForce $[\mathrm{m}]=$ self.loadCellCalibrateVoltage $(($ float $(\operatorname{Code}[0][$ dis+1:volt] $)), y I n t)$

Forcestr[m]=str(self.loadCellCalibrateVoltage((float(Code[0][dis+1:volt])),yInt))+","

dis $=$ Code $[0]$. index (';', dis +1$)$

return[identifyDisp,identifyForce]

\#plot(identifyForce)

\#show()

def

CalibrateCRCNSForce(self,interval,partition,vel,CompleteForce,CompleteDisplacement,forceThresh,POC,highForce,saturation,compForceCount er,failure,feedForward,yInt,socketId, positioner,group,trajectRoute,single,maxForce):

firstTime $=0$

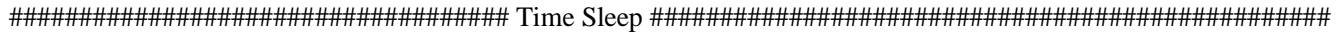

time.sleep (60)

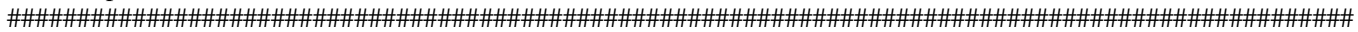

linOut $=$ self.identifyLocation $($ maxForce, socketId, group,yInt,POC,positioner,vel $)$

identifyForce $=$ linOut[1]

identifyDisp = linOut[0]

firstRun = True

expectedDisplacement $=[]$

forceCalibrate $=[]$

val_Greater $=$ False

if(single):

force $=$ highForce

if $(\operatorname{not}($ CompleteForce.__contains__(force $)))$ :

forceCalibrate.append((force))

\#CompleteForce.append([force,vel])

numberOfCalibrate $=$ len (forceCalibrate)

else:

firstContactForceCali $=$ float $($ forceThresh $) / 1000$

saturForceCali $=$ float (saturation)

expo $=[.010, .020, .040, .080, .160, .320, .640]$

if(highForce $<=$ saturForceCali):

if(partition):

for $\mathrm{x}$ in range(len(CompleteForce)):

if $(\operatorname{not}($ CompleteForce $[\mathrm{x}][0]==($ firstContactForceCali $)))$ :

\#CompleteForce.append([(firstContactForceCali),vel])

forceCalibrate.append((firstContactForceCali))

elif $(($ CompleteForce $[\mathrm{x}][0]==($ firstContactForceCali $)$ and $\operatorname{not}($ CompleteForce $[\mathrm{x}][1]==\mathrm{vel})))$ :

\#CompleteForce.append([(firstContactForceCali),vel])

forceCalibrate.append((firstContactForceCali))

for $\mathrm{z}$ in range $(0$, len (expo) $)$ :

if $(($ expo[z] $>$ firstContactForceCali $)$ or $(\operatorname{expo}[z]==$ firstContactForceCali $))$ :

if ((expo[z]<highForce) $)$ :

for $\mathrm{m}$ in range(len(CompleteForce)): 
if $($ not $($ CompleteForce $[\mathrm{m}][0]==(\operatorname{expo}[\mathrm{z}])))$ :

forceCalibrate.append $((\operatorname{expo}[\mathrm{z}]))$

$\operatorname{elif}(($ CompleteForce[m][0] == $(\operatorname{expo}[\mathrm{z}])$ and $\operatorname{not}($ CompleteForce $[\mathrm{m}][1]==\mathrm{vel})))$ :

forceCalibrate.append $((\operatorname{expo}[\mathrm{z}]))$

for $\mathrm{y}$ in range(len(CompleteForce)):

if $(\operatorname{not}($ CompleteForce[y][0] $==($ highForce $)))$ :

forceCalibrate.append(highForce)

elif((CompleteForce $[y][0]==($ highForce $)$ and $\operatorname{not}($ CompleteForce $[y][1]==v e l)))$ :

forceCalibrate.append $((\operatorname{expo[z}]))$

else:

if(not(interval==firstContactForceCali)):

if(not(CompleteForce._contains_([firstContactForceCali,vel] ))):

forceCalibrate.append((firstContactForceCali))

lowerLimit $=$ interval

while(lowerLimit< $<$ highForce):

if(not(CompleteForce._contains_([lowerLimit,vel]))):

forceCalibrate.append(lowerLimit)

lowerLimit $=$ lowerLimit+interval

else:

lowerLimit $=$ lowerLimit+interval

lowerLimit $=$ lowerLimit - interval

if(not(CompleteForce._contains_([highForce,vel] $)))$ :

forceCalibrate.append(highForce)

random.shuffle(forceCalibrate)

numberOfCalibrate $=$ len (forceCalibrate)

else:

val_Greater $=$ True

print "The Maximum Force is greater then the calibrated line, please lower maximum force"

if(not(val_Greater)):

for y in range( 0 ,numberOfCalibrate):

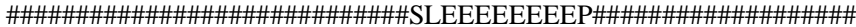

if((forceCalibrate[y])>.001):

time.sleep $(60)$

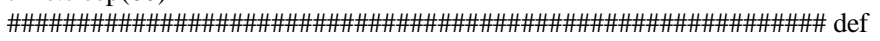

LocationFindCRCNSForce(self,Expectedvoltage,expectedDisplacement,count,vel,socketId,positioner,identifyForce,group,

POC,identifyDisp,feed_forward,firstTime,yInt,trajectRoute ):

\#\#\#\#\#

LFCF=self.LocationFindCRCNSForce(self.loadCellCalibrateForce(forceCalibrate[y],yInt), expectedDisplacement,y,vel,socketId,positioner,identi fyForce, group, POC, identifyDisp, feedForward, firstTime, yInt,trajectRoute,firstRun)

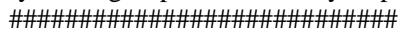

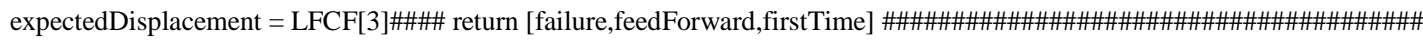

failure $=$ LFCF[0]

feedForward $=$ LFCF[1]

totalTrajTime $=$ LFCF[5]

CompleteForce.append([forceCalibrate[y],expectedDisplacement,vel,failure,feedForward,totalTrajTime])

firstRun $=$ False

\# expectedDisplacement $=\mathrm{LFCF}[3]$

splits $=$ LFCF[4]

\#for $\mathrm{m}$ in range( 0 ,len(expectedDisplacement)):

\# CompleteDisplacement.append(expectedDisplacement[m])

locationArray $=[]$

for $\mathrm{x}$ in range $(0$, len $($ CompleteForce $)$ :

if(CompleteForce[x][3]):

locationArray.append $(\mathrm{x})$

countUp $=0$

for $\mathrm{x}$ in range $(0$, len(locationArray))

$\operatorname{del}($ CompleteForce[locationArray[x]-countUp])

countUp $=$ countUp +1

CompleteForce $=\operatorname{sorted}($ CompleteForce, $\mathrm{key}=$ lambda $\mathrm{x}: \mathrm{x}[0])$

\#CompleteDisplacement=sorted(CompleteDisplacement,key=lambda $\mathrm{x}: \mathrm{x}[1])$

return [CompleteForce,compForceCounter,splits]

def find_nearest(self,array,value):

$\operatorname{idx}=($ np.abs $($ array-value $)) \cdot \operatorname{argmin}()$

return idx

def LocationFindCRCNSForce(self,Expectedvoltage,expectedDisplacement,count,vel,socketId,positioner,identifyForceVal,group,

POC,identifyDispVal,feed_forward,firstTime,yInt,trajectRoute,firstRun):

counter_point $=" "$

\#sleepTime $=0$

\#if(vel ==1):

sleepTime $=1.5$ 
\#else:

\# sleepTime $=1$

if (univ_Var $==$ 'UVA'):

counter_point= "10"

else:

counter_point $=" 8 "$

expectedValue $=0$

breaker=True

expectedValue $=0$

for $\mathrm{x}$ in range(len(identifyForceVal)):

if(identifyForceVal[x]<= self.loadCellCalibrateVoltage(Expectedvoltage,yInt) and identifyDispVal[x] $>0$ ):

expectedValue $=$ identifyDispVal $[\mathrm{x}]$

identifyDisp=[]

identifyForce=[]

identifyDispVal $=$ np.array(identifyDispVal)

indexVal $=$ self.find_nearest $($ identifyDispVal, 0$)$

identifyDisp.append(identifyDispVal[indexVal:len(identifyDispVal)])

identifyForce.append(identifyForceVal[indexVal:len(identifyForceVal)])

forceZero $=$ identifyForce[0][0]

\#print identifyForce

for i in range(len(identifyDisp[0])):

identifyForce[0][i]=identifyForce[0][i]-forceZero

identifyDisp $=$ np.array(identifyDisp)

identifyForce $=$ np.array(identifyForce)

expectedValue $=$ expectedValue $+\mathrm{POC}$

relDisp $=$ expectedValue $-\mathrm{POC}+.6$

expectedDisplacement $=$ expectedValue

chooseFile = 'Calib_force '+str(self.loadCellCalibrateVoltage(Expectedvoltage,yInt) $)+$ ' vel ' +str('\{0:.0f $\}$ '.format(vel) $)+$ '.trj'

leg1_velfinal $=$ vel

leg1_velstart $=0$

leg1_time $=$ float $($ leg1_velfinal-leg1_velstart $) / 80$

leg1_disp = float(leg1_velfinal + leg1_velstart)/2*leg1_time

$\#$ if $(\mathrm{vel}==1)$ :

\# leg3_velfinal $=.0068 * n p . \log ($ self.loadCellCalibrateVoltage $($ Expectedvoltage,yInt $))+.056$

\#elif(vel==2):

\# leg3_velfinal=.0053*np.log(self.loadCellCalibrateVoltage(Expectedvoltage,yInt))+.0484

leg3_velfinal $=0$

leg3_velstart $=$ vel

leg3_time $=$ float $($ leg3_velfinal-leg3_velstart $) /(-80)$

leg3_disp = float $\left(\right.$ leg3_velfinal + leg3_velstart) $/ 2 * \operatorname{leg} 3 \_$time

leg2_velfinal $=$ vel

leg2_disp = float(relDisp)-leg1_disp-leg3_disp

leg2_time = leg2_disp/vel

FILECal $=$ open $($ chooseFile,"a")

FILECal.write(str('\{0:.6f $\}$ '.format(leg1_time))+',' + str('\{0:.6f $\}$ '.format(leg1_disp))+ ','+str('\{0:.6f $\}$ '.format(leg1_velfinal))+'|n')

FILECal.write(str('\{0:.6f\}'.format(leg2_time))+',' + str('\{0:.6f\}'.format(leg2_disp))+ ','+str('\{0:.6f\}'.format(leg2_velfinal))+'|n')

FILECal.write(str('\{0:.6f\}'.format(leg3_time))+',' + str('\{0:.6f $\}$ '.format(leg3_disp))+ ','+str('\{0:.6f $\}$ '.format(leg3_velfinal))+'|n')

\#FILECal.write(str('\{0:.6f\}'.format(.020))+',' + str('\{0..6f\}'.format(.0004))+ ','+str('\{0..6f\}'.format(0))+'In')

FILECal.close()

ftps = ftplib.FTP('192.168.0.254','Administrator','Administrator')

ftps.cwd(trajectRoute)

self.upload(ftps,chooseFile)

ftps.quit()

os.remove(chooseFile)

[errorCode, returnString]=myxps.PositionerSGammaParametersSet(socketId,positioner,vel, $80, .005, .05$ ) 
[errorCode,returnString]=myxps.GatheringReset(socketId)

if(errorCode !=0):

self.displayErrorAndClose (socketId,errorCode,'GatheringReset')

sys.exit()

[errorCode, returnString]=myxps.GatheringConfigurationSet(socketId,[positioner+".CurrentPosition","GPIO2.ADC1"])

if (errorCode != 0):

self.displayErrorAndClose (socketId, errorCode, 'GatheringConfigurationSet')

sys.exit()

[errorCode, returnString]=myxps.EventExtendedConfigurationTriggerSet(socketId,[group+".PVT.TrajectoryStart"],["0"],["0"],["0"],["0"])

\#[errorCode, returnString]=myxps.EventExtendedConfigurationTriggerSet(socketId,[positioner+".SGamma.MotionStart"],["0"],["0"],["0"],["0"])

if (errorCode $!=0$ ):

self.displayErrorAndClose (socketId, errorCode, 'EventExtendedConfigurationTriggerSet')

sys.exit()

if(self.loadCellCalibrateVoltage(Expectedvoltage,yInt)<=.015):

[errorCode, returnString]=myxps.EventExtendedConfigurationActionSet(socketId,["GatheringRun"],["10000"],[counter_point],["0"],["0"])

if (errorCode != 0):

self.displayErrorAndClose (socketId, errorCode, 'EventExtendedConfigurationActionSet')

sys.exit()

else:

[errorCode, returnString]=myxps.EventExtendedConfigurationActionSet(socketId,["GatheringRun"],["10000"],[counter_point],["0"],["0"])

if (errorCode != 0):

self.displayErrorAndClose (socketId, errorCode, 'EventExtendedConfigurationActionSet')

sys.exit()

[errorCode, returnString]=myxps.EventExtendedStart(socketId)

if (errorCode != 0):

self.displayErrorAndClose (socketId, errorCode, 'Event Extended Start')

sys.exit()

[errorCode,returnString]=myxps.MultipleAxesPVTExecution(socketId,group,chooseFile,1)

\#[errorCode, returnString] = myxps.GroupMoveRelative(socketId, group, [relDisp])

time.sleep(sleepTime)

[errorCode,returnString] $=$ myxps. GatheringStopAndSave $($ socketId $)$

if(errorCode! $=0)$ :

self.displayErrorAndClose(socketId,errorCode,'GatheringStopandSave')

sys.exit()

[errorCode, returnString] = myxps.GroupMoveAbsolute(socketId, group, [POC-.6])

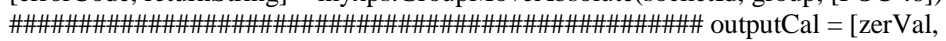

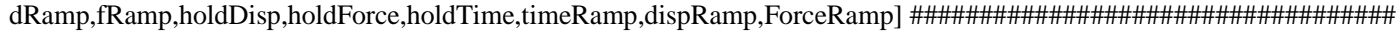

outputCal $=$ self.outputCalib(POC,breaker,expectedValue,self.loadCellCalibrateVoltage(Expectedvoltage,yInt),False, 1,socketId,y Int, vel)

firstTime $=0$

zerVal $=$ outputCal[0]

failure $=$ False

holdTime $=$ outputCal[5]

holdForce $=$ outputCal[4]

fRamp $=$ outputCal[2]

dRamp $=$ outputCal[1]

timeRamp $=$ outputCal[6]

dispRamp =outputCal[7]

assumeVal $=[.008,3, .013,4, .35, .02]$

calcChangeVal $=$ self.calculateChangeVal(zerVal, firstTime, failure,

breaker,expectedValue,Expectedvoltage,holdTime,holdForce,timeRamp,dispRamp,fRamp,dRamp,feed_forward,POC,yInt,vel,socketId,relDisp,ft ps,trajectRoute, group,identifyForce,identifyDisp,assumeVal)

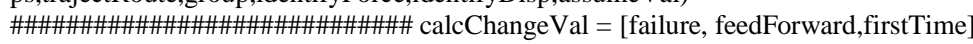

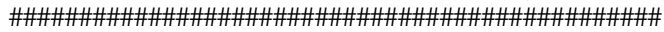

failureVal = calcChangeVal[0]

feedForward $=$ calcChangeVal[1]

firstTime $=$ calcChangeVal[2]

splits $=$ calcChangeVal[3]

totalTrajTime $=$ calcChangeVal[4]

return [failureVal,feedForward,firstTime,expectedDisplacement,splits,totalTrajTime]

def outputCalib(self,POC, breakAp,peakDisp,expectedForce,disp, modValue,socketId,yInt,vel):

zerVal $=$ False

dRamp $=[]$ 
fRamp $=[]$

holdForce $=[]$

holdDisp=[]

holdTime $=[]$

allForce $=[]$

allTime $=[]$

CurrentNumber $=$ myxps.GatheringCurrentNumberGet(socketId) $[1]$

iterations $1=$ CurrentNumber $/ 500$

LeftOver $=$ CurrentNumber $\% 500$

Code $=[]$

$\mathrm{C}=[]$

starting $1=0$

count $1=0$

\#Iterates over the 500 points that the program can read at a maximum time

endCount $=500$

for $\mathrm{y}$ in range(iterations $1+1)$ :

count $1=$ count $1+1$

if $(\mathrm{y}==($ iterations 1$)$ and (LeftOver $!=0)$ ):

$\mathrm{C}=$ [errorCode, returnString $]=$ myxps.GatheringDataMultipleLinesGet(socketId,starting1,LeftOver)

if (errorCode != 0):

self.displayErrorAndClose (socketId, errorCode, 'GatheringDataMultipleLinesGet')

sys.exit()

C.remove (0)

Code $[0]=\operatorname{Code}[0]+C[0]$

elif( $y<$ iterations1):

if $(\mathrm{y}==0)$ :

$\mathrm{C}=[$ errorCode, returnString $]=$ myxps.GatheringDataMultipleLinesGet(socketId,starting1, endCount)

if (errorCode != 0):

self.displayErrorAndClose (socketId, errorCode, 'GatheringDataMultipleLinesGet')

sys.exit()

C.remove(0)

Code.append $(\mathrm{C}[0])$

starting $1=$ endCount + starting 1

else:

$\mathrm{C}=[$ errorCode, returnString $]=$ myxps.GatheringDataMultipleLinesGet(socketId,starting1,endCount)

if (errorCode $!=0$ ):

self.displayErrorAndClose (socketId, errorCode, 'GatheringDataMultipleLinesGet')

sys.exit()

C.remove (0)

Code $[0]=$ Code $[0]+$ C $[0]$

starting $1=$ endCount + starting 1

\#Creates arrays that will moderate over gathered data

$\mathrm{x}=$ Code[0].count (';')

displacement $=[$ None $] * \mathrm{x}$

Force $=[\text { None }]^{*} \mathrm{x}$

Forcestr $=[\text { None }]^{*} \mathrm{x}$

displacementstr $=[\text { None }]^{*} \mathrm{x}$

dis $=$ Code[0].index(';')

volt $=-1$

time $=[]$

timestr $=[]$

for $\mathrm{m}$ in range(x):

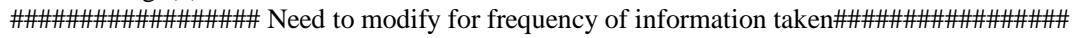

$\mathrm{z}=\mathrm{m}^{*} .001$

time.append(z)

timestr.append(str(z))

if $(m==x-1)$ :

displacement $[\mathrm{m}]=$ float("\%.3f"\%(float(Code[0][volt+1:dis])-(POC)))

displacementstr $[\mathrm{m}]=\operatorname{str}($ float $($ Code $[0][$ volt $+1:$ dis $])-($ POC $))$

volt=Code[0].index ('In',volt+1,len(Code[0]))

Force $[\mathrm{m}]=$ self.loadCellCalibrateVoltage $(($ float $($ Code[0][dis+1:volt $]))$,ylnt $)$

Forcestr[m]=str(self.loadCellCalibrateVoltage((float(Code[0][dis+1:volt])),yInt))+","

else: 
displacement $[\mathrm{m}]=$ float $\left(" \% .3 \mathrm{f}^{\prime \prime} \%(\right.$ float $($ Code $[0][$ volt+1:dis])-(POC))

displacementstr $[\mathrm{m}]=\operatorname{str}($ float $($ Code $[0][$ volt $+1: \mathrm{dis}])-(\mathrm{POC}))$

volt=Code[0].index ('In',volt+1,len(Code[0]))

Force $[\mathrm{m}]=$ self.loadCellCalibrateVoltage $($ float $($ Code[0][dis+1:volt])),yInt $)$

Forcestr[m]=str(self.loadCellCalibrateVoltage((float(Code[0][dis+1:volt])),yInt))+","

dis $=$ Code[0].index(';',dis+1)

forceHolder $=[]$

for $\mathrm{x}$ in range(len(Force)):

if $($ displacement $[\mathrm{x}]>0)$ :

forceHolder.append(Force[x])

else:

forceHolder.append $(0)$

peakerDisp $=1$

location $=0$

if(disp):

valDisp = float $(" \% .2 \mathrm{f} " \%($ peakDisp-.6))

highDisp $=$ displacement.index $(($ valDisp $))$

dispRamp $=$ displacement $[0:$ highDisp $]$

ForceRamp $=$ Force[0:highDisp $]$

timeRamp = time[0:highDisp]

for $\mathrm{x}$ in range(500):

allForce.append(Force[highDisp+x])

allTime.append(time[highDisp $+\mathrm{x}]$ )

if $(x \% \bmod V$ alue $==0)$ :

holdDisp.append(displacement[highDisp+x])

holdForce.append((Force[highDisp $+\mathrm{x}]))$

holdTime.append(time[highDisp+x])

counter $=0$

holdDisp $=$ np.array (holdDisp)

holdForce $=$ np. .array $($ holdForce $)$

holdTime $=$ np.array $($ holdTime $)$

if(breakAp):

for $\mathrm{i}$ in range(len(dispRamp)):

if (dispRamp[i] $>0$ and ForceRamp[i] $>$ float(expectedForce) $/ 2$ and counter $<1)$ :

location $=\mathrm{i}$

counter $=$ counter +1

dRamp.append(dispRamp[location:len(dispRamp)])

fRamp.append(ForceRamp[location:len(dispRamp)])

$\mathrm{dRamp}=\mathrm{dRamp}[0]$

$\mathrm{fRamp}=\mathrm{fRamp}[0]$

else:

plt.figure(1)

plt.subplot(211)

plt.plot(time,displacement,'b')

plt.ylim $(0, .3)$

plt.subplot(212)

plt.plot(time,Force,'b')

plt.ylim $(0, .075)$

plt.plot()

\#if(vel ==1):

totalCells $=1500$

\#else:

$\#$ totalCells $=1000$

highDisp = len(time) - totalCells

if $($ not $($ peakerDisp==0)):

dispRamp $=$ displacement [0:highDisp $]$

counter $=0$

ForceRamp $=$ Force[0:highDisp]

for $i$ in range(len(dispRamp)): 


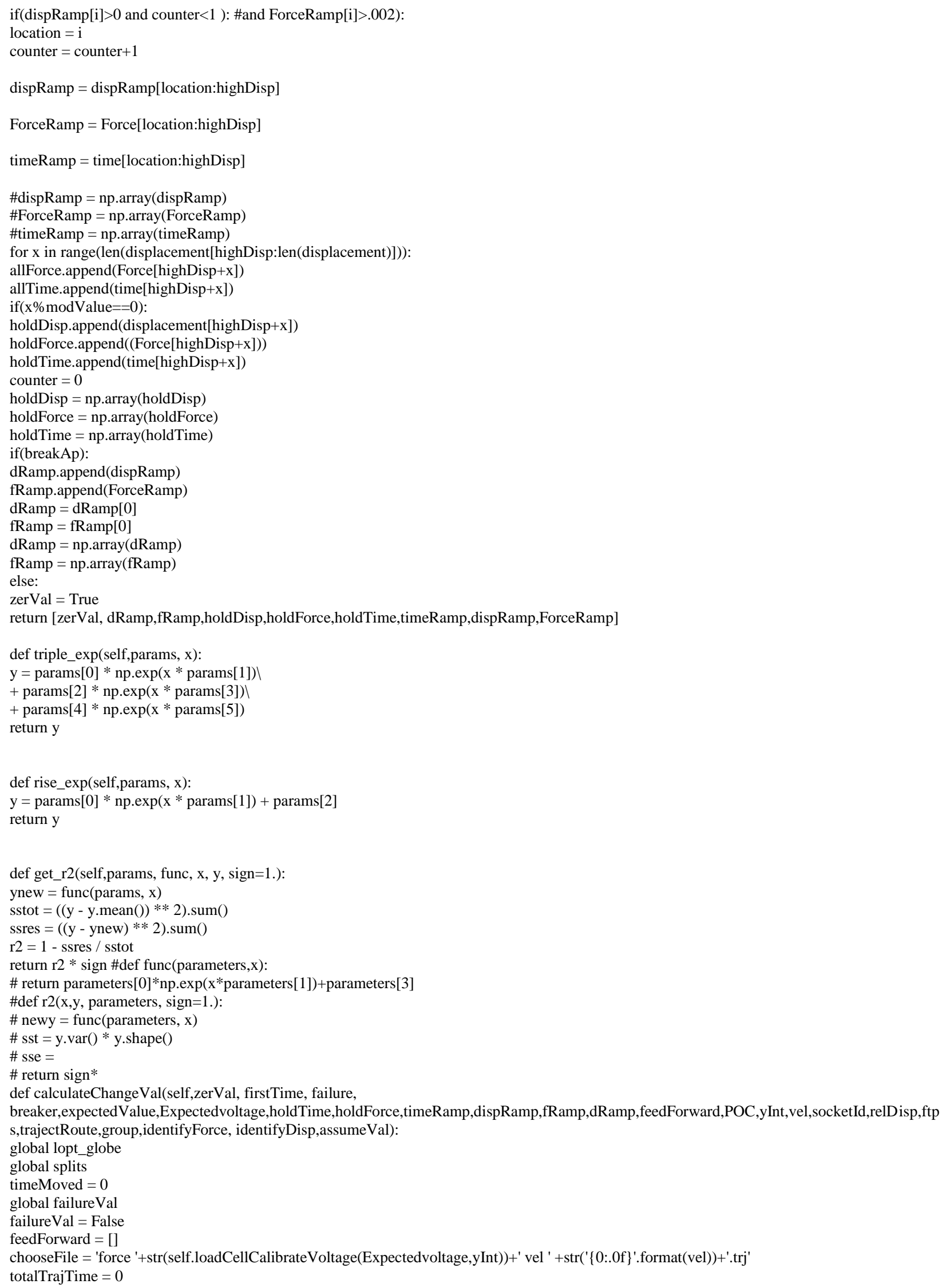


chooseFileDisp = 'disp '+str(expectedValue)+' vel ' +str('\{0:.0f $\}$ '.format(vel))+'.trj'

if(not(failure)):

try:

os.remove(chooseFile)

except OSError:

pass

try:

os.remove(chooseFileDisp)

except OSError:

pass

if(not(zerVal)):

\#hyper_displ = identifyDisp[0]

\#hyper_force $=$ identifyForce[0]

\#hyper_displ = np.array(hyper_displ)

\#hyper_force $=$ np.array (hyper_force)

hyper_displ $=$ dRamp

hyper_force $=$ fRamp

hyper_displ $=$ np.array $($ hyper_displ)

hyper_force $=$ np.array(hyper_force)

$\# \mathrm{x} 0=$ np.array([hyper_force.mean(), 1., -hyper_force.mean()]

\#bounds $=((0$, None $),(0$, None $),($ None, None $))$

\#res $=$ minimize (

\# self.get_r2, x0, args=(self.rise_exp, hyper_displ, hyper_force, -1),

\# method='SLSQP', bounds=bounds)

\#hyper_param $=$ res.x

\#lopt $=$ hyper_param

\#lopt_2,lcov = curve_fit(self.fitfuncPosExpo,identifyDisp[0],identifyForce[0],p0=(.007,13.9,-.003))

\#lopt,lcov = curve_fit(self.fitfuncPosExpo,identifyDisp[0],identifyForce[0],p0=(hyper_force.mean(),13.9,-hyper_force.mean()))

lopt,lcov = curve_fit(self.fitfuncPosExpo,dRamp,fRamp,p0=(hyper_force.mean(),13.9,-hyper_force.mean()),maxfev=3000)

lopt_globe.append([expectedValue,vel,lopt[0],lopt[1],lopt[2]])

$\mathrm{yD}=[]$

$\# \mathrm{yD} \_2=[]$

for y in range $(0$,len (dRamp)):

\#yD_2.append(self.fitfuncPosExpo(identifyDisp[0][y],lopt_2[0],lopt_2[1],lopt_2[2]))

\#yD.append(self.fitfuncPosExpo(dRamp,lopt[0],lopt[1],lopt[2]))

yD.append(lopt[0]*np.exp(dRamp[y]*lopt[1])+lopt[2])

plt.figure(2)

plt.plot(dRamp,fRamp,'b')

plt.plot $\left(\mathrm{dRamp}, \mathrm{yD}, \mathrm{r}^{\prime}\right.$, linewidth $\left.=2.0\right)$

plt. $\operatorname{ylim}(0, .075)$

plt.show()

plt.figure(3)

plt.subplot(211)

plt.plot(timeRamp,fRamp,'b')

plt.ylim $(0, .075)$

plt.subplot(212)

plt.plot(timeRamp,dRamp,'b')

plt.ylim(0,7)

plt.show()

\#plt.plot(identifyDisp[0],yD_2,'g')

\#plt.show()

\#except RuntimeError:

$\#$ lopt $=[0,0,0]$

\# if(not(firstRun)):

\# for $\mathrm{x}$ in range(len(lopt_globe)):

\# if(lopt_globe[x][1]==vel):

$\#$

$\#$ lopt $[0]=$ lopt_globe[0][2]

$\#$ lopt[1] = lopt_globe[0][3]

\# lopt[2] = lopt_globe[0][4]

\# else:

\# lopt,lcov = curve_fit(self.fitfuncPosExpo,identifyDisp[0],identifyForce[0],p0=(.007,13.9,-.003))

\#print "runtime lopt:" + str(lopt)

\# lopt_globe.append([expectedValue,vel,lopt[0],lopt[1],lopt[2]])

\# $\mathrm{yD}=[]$

\# for y in range( 0 ,len(dRamp)):

\#yD.append(self.fitfuncPosExpo(identifyDisp[0][y],lopt[0],lopt[1],lopt[2])) 
\# yD.append(self.fitfuncPosExpo(dRamp,lopt[0],lopt[1],lopt[2]))

try:

offsetForce $=\max ($ holdForce $)$

diffForce $=$ self.loadCellCalibrateVoltage (Expectedvoltage,yInt)-offsetForce

for $\mathrm{x}$ in range(len(holdForce)):

holdForce $[\mathrm{x}]=$ holdForce $[\mathrm{x}]+$ diffForce

holdTime $=($ holdTime-holdTime $[0])$

holdForce $=$ holdForce

if(self.loadCellCalibrateVoltage(Expectedvoltage,yInt)<=.02):

startingPoint $=$

[self.loadCellCalibrateVoltage(Expectedvoltage,yInt)*.85,12,self.loadCellCalibrateVoltage(Expectedvoltage,yInt)*.1,1.5,self.loadCellCalibrateV oltage(Expectedvoltage,yInt)*.05,.02]

else:

startingPoint $=$

[self.loadCellCalibrateVoltage(Expectedvoltage,yInt)*.85,16,self.loadCellCalibrateVoltage(Expectedvoltage,yInt)*.1,4,self.loadCellCalibrateVol tage(Expectedvoltage, yInt)*.05,.012]

$\# \mathrm{x} 0=\operatorname{np} . \operatorname{array}([1,-1,1,-1,1,-1]) *$ holdForce.max ()

$\# \mathrm{x} 0=$ np.array([self.loadCellCalibrateVoltage(Expectedvoltage,yInt)*.85,16,self.loadCellCalibrateVoltage(Expectedvoltage,yInt)*.1,4,self.loadCe 11CalibrateVoltage(Expectedvoltage,yInt)*.05,.012])

\#bounds $=((0$, None $),($ None, 0$)$,

\# (0, None), (None, 0$)$,

$\#(0$, None), (None, 0$))$

\#res = minimize

\# self.get_r2, x0, args=(self.triple_exp, holdTime, holdForce, -1$)$,

\# method='SLSQP', bounds=bounds)

\#triple_exp_params = res.X

\#print triple_exp_params

\#tripopt $=$ triple_exp_params

altTripFit $=[]$

tripopt,tripcov $=$ curve_fit(self.fitfuncTrip, holdTime, holdForce, $\mathrm{p} 0=$

(startingPoint[0],startingPoint[1],startingPoint[2],startingPoint[3],startingPoint[4],startingPoint[5]), maxfev = 3000)

\#print tripopt

\#tripFit =[]

tripFit $=[]$

negTripFit $=[]$

for y in range(0,len(holdTime)):

tripFit.append(self.fitfuncTrip(holdTime[y],tripopt[0],tripopt[1],tripopt[2],tripopt[3],tripopt[4],tripopt[5]))

\#altTripFit.append(triple_exp_params[0]*math.exp(holdTime[y]*-triple_exp_params[1])+triple_exp_params[2]*math.exp(holdTime[y]*-

triple_exp_params[3])+triple_exp_params[4]*math.exp(holdTime[y]*-triple_exp_params[5]))

negTripFit.append((self.fitfuncTrip(holdTime[y],-tripopt[0],tripopt[1],-tripopt[2],tripopt[3],-tripopt[4],tripopt[5])))

tripFit[0]

negTripFit[0]

difference $=$ tripFit $[0]$-negTripFit $[0]$

for $\mathrm{n}$ in range $(0$,len(holdTime)):

negTripFit[n] = negTripFit[n]+difference

\#plt.plot(holdTime,holdForce)

\#plt.plot(holdTime,tripFit,'r')

\#plt.show()

\#plt.plot(holdTime,altTripFit,'g')

tripoptVals $=[]$

tripoptVals.append(tripopt[1])

tripoptVals.append(tripopt[3])

tripoptVals.append(tripopt[5])

tripoptVals $=$ sorted(tripoptVals)

splits.append([expectedValue,vel,1/tripoptVals[1]/.001])

firstMove $=1 /$ tripoptVals[2]/.001

timeStoppers $=1 /$ tripoptVals[1]/.001

timeStop $=$ timeStoppers $\# / .001$

negyaj $=[]$

for $\mathrm{x}$ in range $(0$, len(tripFit $))$ :

negyaj.append $((\operatorname{math} \cdot \log ((\operatorname{tripFit}[\mathrm{x}]-\operatorname{lopt}[2]) /(\operatorname{lopt}[0])) / \operatorname{lopt}[1]))$

$\# \mathrm{x} 0=$ np.array([assumeVal[0],tripopt[1], assumeVal[2],tripopt[3], assumeVal[4],tripopt[5]])

\#bounds $=((0$, None $),($ None, 0$)$,

\# ( 0 , None), (None, 0$)$,

\# (0, None), (None, 0$))$

\#res $=$ minimize

\# self.get_r2, x0, args=(self.triple_exp, holdTime, holdForce, -1$)$,

\# method='SLSQP', bounds=bounds)

\#triple_exp_params.append(res.x)

\#dopt $=$ triple_exp_params 
\#dopt,dcov = curve_fit(self.fitfuncTrip,holdTime,negyaj,p0 = (assumeVal[0],tripopt[1],assumeVal[2],tripopt[3],assumeVal[4],tripopt[5] $)$ ,maxfev=3000)

dispHold $=[]$

dispHold $1=[]$

zerVal $=0$

for $\mathrm{x}$ in range(0,len(holdTime)):

\#dispHold1.append(math.log((tripopt[0]*math.exp(-tripopt[1]*holdTime[x])+tripopt[2]*math.exp(-

tripopt[3]*holdTime[x])+tripopt[4]*math.exp(-tripopt[5]*holdTime[x])-lopt[2])/lopt[0])/lopt[1]) \#self.fitfuncTrip(holdTime[x],-dopt[0],dopt[1],$\operatorname{dopt}[2], \operatorname{dopt}[3],-\operatorname{dopt}[4], \operatorname{dopt}[5])) \#$ offsetVal))\#-dopt[2],dopt[3],offsetVal))

dispHold1.append(math.log $(($ tripopt$[0] *$ math.exp(-tripopt[1]*holdTime[x])+tripopt[2]*math.exp(-tripopt[3]*holdTime[x])+tripopt[4]*math.exp(tripopt[5]*holdTime[x]))/lopt[0]+1)/lopt[1]) \#self.fitfuncTrip(holdTime[x],-dopt[0],dopt[1],-dopt[2],dopt[3],-dopt[4],dopt[5]))\#offsetVal))\#$\operatorname{dopt}[2], \operatorname{dopt}[3]$, offsetVal))

if $(\mathrm{x}==0)$ :

zerVal $=$ dispHold $1[0]$

dispHold.append(-dispHold1[x]+zerVal+zerVal)

plt.figure(4)

plt.plot(holdTime,dispHold)

\#plt.plot(holdTime,dispHold1)

plt.show()

$\operatorname{def} f(x)$ :

return -math.log $((\operatorname{tripopt}[0] *$ math.exp(-tripopt[1]*x)+tripopt[2]*math.exp(-tripopt[3]*x)+tripopt[4]*math.exp(-

tripopt[5]*x))/lopt[0]+1)/lopt[1]+zerVal+zerVal

\#return -math.log((tripopt[0]*math.exp(-tripopt[1]*x)+tripopt[2]*math.exp(-tripopt[3]*x)+tripopt[4]*math.exp(-tripopt[5]*x)-

lopt[2])/lopt[0])/lopt[1]+zerVal+zerVal

\#print str(derivative(f,holdTime[0],dx $=1 \mathrm{e}-3)$ )

\#print $\operatorname{str}($ derivative $(f$, holdTime[2],dx $=1 \mathrm{e}-3)$ )

\#velocityVal $=[-((-\operatorname{dopt}[0] *(\operatorname{dopt}[1])) * \operatorname{math} \cdot \exp (((\operatorname{holdTime}[0]) *(-\operatorname{dopt}[1])))+(-\operatorname{dopt}[2] *(\operatorname{dopt}[3])) * \operatorname{math} \cdot \exp (\operatorname{holdTime}[0] *-\operatorname{dopt}[3])+(-$ $\operatorname{dopt}[4] *(\operatorname{dopt}[5])) *$ math.exp(holdTime[0]*-dopt[5]))]

\#velocityVal =

$\left[1 /\left(\right.\right.$ lopt $[1] *\left(\operatorname{tripopt}[0][0]^{*}\right.$ math.exp(tripopt[0][1]*holdTime[0])+tripopt[0][2]*math.exp(tripopt[0][3]*holdTime[0])+tripopt[0][4]*math.exp(tripo pt[0][5]*holdTime[0])+lopt[2]))]

velocityVal $=[$ derivative $(\mathrm{f}$, holdTime $[0], \mathrm{dx}=1 \mathrm{e}-3)]$

\#holdTime $=$ holdTime[0:timeStop]

totalMove $=[]$

for $\mathrm{x}$ in xrange(1,len(holdTime)):

timeMoved $=$ timeMoved +.001

\#totalMove.append(-dopt[0]*math.exp $((($ timeMoved $) *(\operatorname{dopt}[1])))-\operatorname{dopt}[2] *$ math.exp((timeMoved*(dopt[3])))-

$\operatorname{dopt}[4] *$ math.exp $(($ timeMoved* $(\operatorname{dopt}[5]))))$

totalMove.append(dispHold[x])

\#velocityVal.append(-((-dopt[0]*dopt[1])*math.exp((timeMoved)*(-dopt[1]))+(-dopt[2]*(dopt[3]))*math.exp(timeMoved*-dopt[3])+($\operatorname{dopt}[4] *(\operatorname{dopt}[5])) *$ math.exp(timeMoved*-dopt[5])))

\#velocityVal.append(1/(lopt[1]*(tripopt[0][0]*math.exp(tripopt[0][1]*holdTime[x])+tripopt[0][2]*math.exp(tripopt[0][3]*holdTime[x])+tripopt[ $0][4] *$ math.exp(tripopt[0][5]*holdTime[x])+lopt[2])))

velocityVal.append(derivative(f,holdTime[x],dx = 1e-3))

dispMove $=[]$

timeMove $=[]$

velMove $=[0] * \operatorname{len}($ dispMove $)$

num $=$ np.linspace $(1,10$, num $=10)$

movementArray $=[]$

velArray $=[]$

for $\mathrm{x}$ in range(1,len(totalMove)

movementArray.append(totalMove[x]-totalMove[x-1])

velArray.append(velocityVal[x])

for $\mathrm{x}$ in range $(0, \mathrm{len}(\mathrm{num}))$

if $(x==0)$ :

\#

mover $=$ totalMove $[\operatorname{int}($ num $[\mathrm{x}])]-$ totalMove $[0]$

timeDiff $=\operatorname{num}[\mathrm{x}]$

else:

mover $=$ totalMove $[\operatorname{int}(\operatorname{num}[\mathrm{x}])-1]-$ totalMove $[\operatorname{int}(\operatorname{num}[\mathrm{x}])-\operatorname{int}(\operatorname{num}[\mathrm{x}-1])-1]$

timeDiff $=$ num $[\mathrm{x}]-$ num $[\mathrm{x}-1]$

if $($ mover $<0)$ :

mover $=-$ mover

dispMove.append(mover)

timeMove.append(timeDiff/1000) 
velMove.append(velocityVal[int(num[x])-1])

leg1_velfinal $=$ vel

leg1_velstart $=0$

leg1_time $=$ float $($ leg1_velfinal-leg1_velstart $) / 80$

leg1_disp = float(leg1_velfinal + leg1_velstart)/2*leg1_time

leg3_velfinal $=$ velocity $\mathrm{Val}[0]$

leg3_velstart = vel

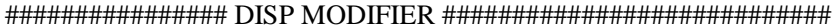

leg3_time = float(leg3_velfinal-leg3_velstart $) /-80$

leg3_disp = float(leg3_velfinal + leg3_velstart) $/ 2 * \operatorname{leg} 3 \_$time

leg2_velfinal $=$ vel

mult_ratio $=1$

leg2_disp = float(relDisp*mult_ratio)-leg1_disp-leg3_disp

\#if(vel==2):

\# leg2_disp = leg2_disp*1.004

\#

\#elif(vel==3):

\# leg2_disp = leg2_disp*1.006

\#elif(vel==4):

\# leg2_disp = leg2_disp*1.008

\#elif(vel==5):

\# leg2_disp = leg2_disp*1.01

leg2_time = leg2_disp/vel

global trajectTimeAll

trajectTime $=[]$

trajectTime.append $(0)$

trajectTime.append(leg1_time)

trajectTime.append(leg1_time+leg2_time)

for $\mathrm{x}$ in range(0,len(num)):

if $(x==0)$ :

trajectTime.append(leg1_time+leg2_time+leg3_time)

else:

trajectTime.append(leg1_time+leg2_time+leg3_time+num[x]/1000)

trajectTimeAll.append([self.loadCellCalibrateVoltage(Expectedvoltage,yInt),trajectTime])

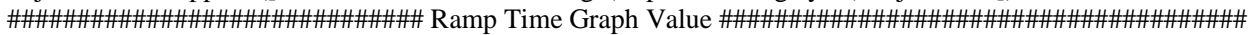

global rampTimeGraph

rampTimeGraph= leg1_time+leg2_time+leg3_time

\#print rampTimeGraph

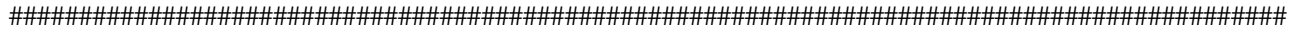

FILE = open(chooseFile,"a")

FILE.write(str('\{0:.6f\}'.format(leg1_time))+',' + str('\{0:.6f\}'.format(leg1_disp))+ ','+str('\{0:.6f\}'.format(leg1_velfinal))+'|n')

FILE.write(str('\{0:.6f\}'.format(leg2_time))+',' + str('\{0:.6f\}'.format(leg2_disp))+ ','+str('\{0:.6f\}'.format(leg2_velfinal))+'In')

FILE.write(str('\{0:.6f\}'.format(leg3_time))+',' + str('\{0:.6f\}'.format(leg3_disp))+ ','+str('\{0:.6f\}'.format(leg3_velfinal))+'In')

countUp $=0$

for $\mathrm{m}$ in range (0,len(movementArray)-1):

FILE.write(str('\{0:.6f $\}$ '.format $(.001))++^{\prime}, '+\operatorname{str}(\{0: .6 f\}$ '.format (movementArray[m]))+ ','+str('\{0:.6f\}'.format(velArray[m+1]))+'ln')

countUp = countUp+.001

FILE.write(str('\{0:.6f\}'.format(.005))+',' + str('\{0:.6f\}'.format(movementArray[len(movementArray)-1]))+ ','+str('\{0:.6f $\}$ '.format(0))+'In')

totalTrajTime $=$ countUp+.005+rampTimeGraph

FILEDisp = open(chooseFileDisp,"a")

FILEDisp.write(str('\{0:.4f\}'.format(leg1_time))+',' + str('\{0:.4f\}'.format(leg1_disp))+ ','+str('\{0:.4f\}'.format(leg1_velfinal))+'In')

FILEDisp.write(str('\{0:.4f\}'.format(leg2_time))+',' + str('\{0:.4f\}'.format(leg2_disp))+ ','+str('\{0:.4f\}'.format(leg2_velfinal))+'In')

FILEDisp.write(str('\{0:.4f\}'.format(leg3_time))+',' + str('\{0:.4f\}'.format(leg3_disp))+ ','+str('\{0:.4f $\}$ '.format $(0))+' \ln$ ')

feedForward $=[$ chooseFile, chooseFileDisp $]$

FILE.close()

FILEDisp.close()

ftps = ftplib.FTP('192.168.0.254','Administrator','Administrator')

ftps.cwd(trajectRoute)

self.upload(ftps,chooseFile)

self.upload(ftps,chooseFileDisp)

ftps.quit()

os.remove(chooseFileDisp)

failureVal $=$ False

[errorCode,returnString]=myxps.MultipleAxesPVTVerification(socketId,group,chooseFile)

if (errorCode $!=0$ ):

failureVal $=$ True

if(leg3_velfinal>vel):

print "Failed Calibration for " + str(self.loadCellCalibrateVoltage(Expectedvoltage,yInt)) + "at " + str(vel) + " mm/s. Please calibrate at a

GREATER velocity to add to table." 
else:

print "Failed Calibration for " + str(self.loadCellCalibrateVoltage(Expectedvoltage,yInt)) + "at " + str(vel) + " mm/s. Please calibrate at a

LESSER velocity to add to table."

except RuntimeError:

if(firstTime<1):

print "Failed Calibration Force Decay for " + str(self.loadCellCalibrateVoltage(Expectedvoltage,yInt))+", running again"

firstTime $=$ firstTime +1

assumeVal $=\left[\operatorname{assumeVal}[0]^{*} .9,10\right.$, assumeVal[2]*.9,4,assumeVal[4]*.9,.02]

self.calculateChangeVal(zerVal,firstTime,failure,

breaker,expectedValue,Expectedvoltage,holdTime,holdForce,timeRamp,dispRamp,fRamp,dRamp,feedForward,POC,yInt,vel,socketId,relDisp,ftp $\mathrm{s}$, trajectRoute,group,identifyForce, identifyDisp,assumeVal)

else:

print "Failed calibration for " + str(self.loadCellCalibrateVoltage(Expectedvoltage,yInt))

failure=True

self.calculateChangeVal(zerVal,firstTime,failure,

breaker,expectedValue,Expectedvoltage,holdTime,holdForce,timeRamp,dispRamp,fRamp,dRamp,feedForward,POC,yInt,vel,socketId,relDisp,ftp $\mathrm{s}$, trajectRoute,group,identifyForce,identifyDisp,assumeVal)

\#print "do i get here"

\#return [failure, feedForward,firstTime,splits]

else:

failureVal $=$ True

return [failureVal, feedForward,firstTime,splits,totalTrajTime]

def fitfuncLog(self, $x, a, b)$ :

return $a^{*} n p \cdot \log (\mathrm{x})+\mathrm{b}$

def fitfuncLine(self, $x, a, b)$ :

return $a^{*}(\mathrm{x})+\mathrm{b}$

def fitfuncExpo(self, $x, a, b)$ :

return a*np.exp(-b*x)

def fitfuncExpo2(self, $x, a, b, c)$ :

return $a * n p \cdot \exp (-b * x)+c$

def fitfuncPosExpo(self, $\mathrm{x}, \mathrm{a}, \mathrm{b}, \mathrm{c})$ :

return $a^{*} n p \cdot \exp \left(b^{*} x\right)+c$

def fitfuncDub(self,x,a,b,c,d):

return $a^{*} n p \cdot \exp (-b * x)+c * n p \cdot \exp \left(-d^{*} x\right)$

def fitfuncTrip(self,x,a,b,c,d,e,f):

return $a^{*} n p \cdot \exp \left(-b^{*} x\right)+c^{*} n p \cdot \exp \left(-d^{*} x\right)+e^{*} n p \cdot \exp \left(-f^{*} x\right)$

def fitfuncTripC(self,x,a,b,c,d,e,f,g):

return $a^{*} n p \cdot \exp \left(-b^{*} x\right)+c * n p \cdot \exp \left(-d^{*} x\right)+e^{*} n p \cdot \exp \left(-f^{*} x\right)+g$

def fitfuncExpoQuad(self,x,a,b,c):

return $\mathrm{a} * \mathrm{x} * 2+\mathrm{b} * \mathrm{x}+\mathrm{c}$

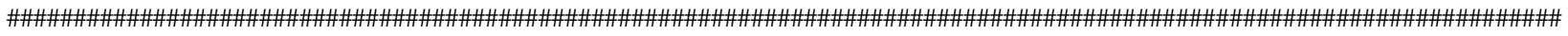
\#\#\#\#\#\#\#\#\#\#\#

\#

\# Force Control Indentation

\#

myIndenter.RunIndentation(velocity,displacementExp,AccMax,Dur,expVol,CompleteForce[ActInt][0],forceActStr,forceActVal,POC,feedForwar d,positioner,socketId,group,fileLoc,yInt)

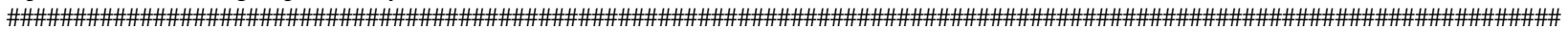
\#

def RunIndentation(self,velocity, expDisp , Acceleration,

Duration,expVol,Force,forceInd,POC,feedForward,positioner,socketId,group,fileLoc,yInt,totalTrajTime,skin_Thickness):

global univ_Var

counter_point $=" "$

if(univ_Var == 'UVA'):

counter_point $=$ "10"

else:

counter_point $=$ "8"

expDisp = float (expDisp)

$\operatorname{expAcc}=$ float (Acceleration)

$\operatorname{expDur}=$ float $($ Duration $)$ 
myxps.GroupMoveAbsolute(socketId,group,[POC-.6])

[errorCode,returnString]=myxps.GatheringReset(socketId)

if (errorCode != 0):

self.displayErrorAndClose (socketId, errorCode, 'GatheringReset')

sys.exit()

[errorCode, returnString]=myxps.GatheringConfigurationSet(socketId,[positioner+".CurrentPosition","GPIO2.ADC1"])

if (errorCode != 0):

self.displayErrorAndClose (socketId, errorCode, 'GatheringConfigurationSet')

sys.exit()

[errorCode, returnString]=myxps.EventExtendedConfigurationTriggerSet(socketId,[group+".PVT.TrajectoryStart"],["0"],["0"],["0"],["0"])

if (errorCode $!=0$ ):

self.displayErrorAndClose (socketId, errorCode, 'EventExtendedConfigurationTriggerSet')

sys.exit()

\#\#\#\#\#\#\#\#\#\#\#\#\#\#\#\#\#\#\#\#\#\#\#\#\#\#\#\#\#\#\#\#\#\#\#\#\#\#\#\#\#\#\#\#\#\#\#\#\#\#\#\#\#\#\#\#\# Change

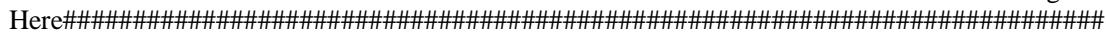

[errorCode, returnString]=myxps.EventExtendedConfigurationActionSet(socketId,["GatheringRun"],["2000"],["50"],["0"],["0"])

if (errorCode !=0):

self.displayErrorAndClose (socketId, errorCode, 'EventExtendedConfigurationActionSet')

sys.exit()

[errorCode, returnString]=myxps.EventExtendedStart(socketId)

if (errorCode ! 0 ):

self.displayErrorAndClose (socketId, errorCode, 'Event Extended Start')

sys.exit()

self.runForce_indent(expDisp,expVol,velocity,expDur,expAcc,Force,forceInd,POC,group,feedForward,positioner,socketId,yInt,totalTrajTime,ski n_Thickness)

[errorCode, returnString] $=$ myxps. GatheringStopAndSave $($ socketId $)$

if (errorCode $!=0$ ):

self.displayErrorAndClose (socketId, errorCode, 'GatheringStopandSave')

sys.exit()

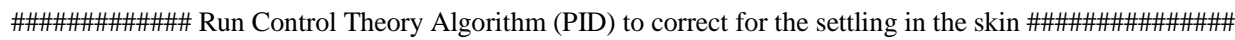
def

runForce_indent(self,expDisp,expectedVoltage,velocity,expDur,expAcc,Force,forceInd,POC,group,feedForward,positioner,socketId,yInt,totalTra jTime,skin_Thickness):

lopt_globe_val $=$ []

for $\mathrm{x}$ in range(len(lopt_globe)):

if(lopt_globe[x][0]==expDisp and lopt_globe[x][1]==velocity):

lopt_globe_val.append(lopt_globe[x][2])

lopt_globe_val.append(lopt_globe[x][3])

lopt_globe_val.append(lopt_globe[x][4])

fileName $=$ feedForward $[0]$

Integrator_max $=500$

Integrator_min $=-500$

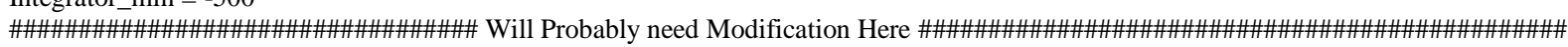

if $($ velocity $==1$ or velocity $==2$ or velocity $==.5$ ):

Pmult $=.005$

Dmult $=.00005$

IMult $=.0005$

if $($ velocity $==3$ or velocity $==4)$ :

Pmult $=.005$

Dmult $=.00005$

IMult $=.0005$

if $($ velocity $==5)$ :

Pmult $=.002$

Dmult $=.0008$

IMult $=.00005$

Pmult $=$ Pmult*skin_Thickness

Dmult $=$ Dmult $*$ skin_Thickness 
Integrator $=0$

Integrator_max $=1 * 10 * *$-6/IMult

LastError $=0$

time_to_stop $1=$ False

count $=0$

[errorCode, returnString]=myxps.PositionerSGammaParametersSet(socketId,positioner, 1,100,.005,.05)

\#[errorCode, returnString]=myxps.PositionerSGammaParametersSet(socketId,positioner,.5, 100,.005,.01)

\# startTime1 = datetime.datetime.now ()

[errorCode,returnString]=myxps.MultipleAxesPVTExecution(socketId,group,fileName,1)

expectedForce $=$ self.loadCellCalibrateVoltage $($ myxps.GPIOAnalogGet(socketId,["GPIO2.ADC1"])[1],yInt $)$

\#print expectedForce

\# stopTime1 $=$ datetime.datetime.now ()

\# difference $=$ stopTime1-startTime1

\# difference $=$ divmod $($ difference.total_seconds ()$, 60)$

\# differenceVal = totalTrajTime - difference[1]

startTime $=$ datetime. datetime.now ()

stopTime $=$ startTime + datetime $\cdot$ timedelta $($ seconds $=\operatorname{expDur})$

prevTime $=$ datetime. datetime.now ()

dtprev $=0$

while(not(time_to_stop1)):

now $=$ datetime.datetime.now ()

if(stopTime<now):

time_to_stop1 = True

$\mathrm{dt}=$ now - prevTime

$\mathrm{dt}=$ divmod(dt.total_seconds ()$, 60)$

dtprev $=\mathrm{dt}[1]+\mathrm{dtprev}$

error $=($ math.log $(($ expectedForce-lopt_globe_val[2] $) /$ lopt_globe_val[0] $) /$ lopt_globe_val[1] $)$ -

(math.log((self.loadCellCalibrateVoltage(myxps.GPIOAnalogGet(socketId,["GPIO2.ADC1"])[1],yInt)-

lopt_globe_val[2])/lopt_globe_val[0])/lopt_globe_val[1])

\#print "error: "+ str(error)

$\mathrm{P}$ _value $=$ Pmult $*$ error

$\operatorname{if}(\operatorname{not}(\mathrm{dt}[1]==0))$ :

D_value $=$ Dmult $*(($ error-LastError $)) / \mathrm{dt}[1]$

else:

D_value $=0$

Integrator $+=$ error*dt[1]

if Integrator > Integrator_max:

Integrator $=$ Integrator_max

\# elif Integrator < Integrator_min:

\# Integrator $=$ Integrator_min

I_value $=$ Integrator $*$ IMult

PID = P_value + I_value + D_value

\#print "P_value: " + str(P_value $)$

\#print "I_value: " + str(I_value)

\#print "D_value: " + str(D_value)

LastError $=$ error

prevTime $=$ datetime.datetime.now ()

\#print PID

[errorCode, returnString] = myxps.GroupMoveRelative $($ socketId, group, $[(\mathrm{PID})])$

\#print "PID" + str(PID)

\#\#

$\#$ if $($ count $==0)$ :

\# stopTime1 = datetime.datetime.now ()

$\#$ endBeg = stopTime1-startTime 1

\#print "first move time" + str(divmod(endBeg.total_seconds(),60)[1])

$\#$ count $=1$

\#if((voltage>8)) 
\# myxps.GroupMoveAbsolute(socketId,group,[POC-.6])

\#print "values" + str(dtprev)

[errorCode, returnString]=myxps.PositionerSGammaParametersSet(socketId,positioner,velocity,80,.005,.05)

[errorCode, returnString] = myxps.GroupMoveAbsolute(socketId, group,[POC-.6])

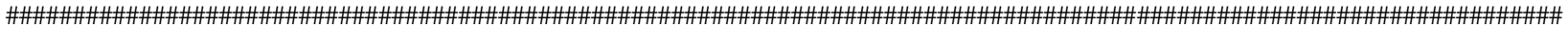

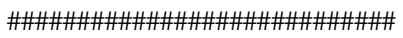

\#\#\#\#\#\#\#\#\#\#\#\#\#\#\#\#\#\#\#\#\#\#\#\#\#\#\#\#\#\#\#\#\#\#\# Displacement Control in Step 6

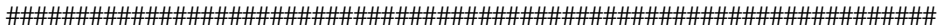

\#

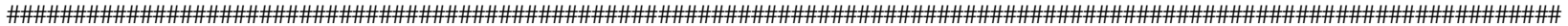

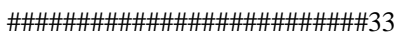

def dispForce(self,vel,holdDur,dispInd,feedForward,socketId,positioner,group,POC,disp):

global univ_Var

counter_point $=" "$

if(univ_Var == 'UVA'):

counter_point= "10"

else:

counter_point $=" 8 "$

fileName $=$ feedForward[1]

[errorCode,returnString]=myxps.GatheringReset(socketId)

if (errorCode != 0):

self.displayErrorAndClose (socketId, errorCode, 'GatheringReset')

sys.exit()

[errorCode, returnString]=myxps.GatheringConfigurationSet(socketId,[positioner+".CurrentPosition","GPIO2.ADC1"])

if (errorCode != 0):

self.displayErrorAndClose (socketId, errorCode, 'GatheringConfigurationSet')

sys.exit()

[errorCode, returnString]=myxps.EventExtendedConfigurationTriggerSet(socketId,[positioner+".SGamma.MotionStart"],["0"],["0"],["0"],["0"])

if (errorCode $!=0$ ):

self.displayErrorAndClose (socketId, errorCode, 'EventExtendedConfigurationTriggerSet')

sys.exit()

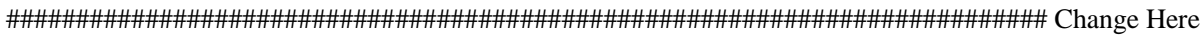

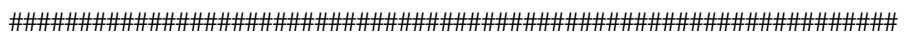

[errorCode, returnString]=myxps.EventExtendedConfigurationActionSet(socketId,["GatheringRun"],["2000"],["50"],["0"],["0"])

if (errorCode != 0):

self.displayErrorAndClose (socketId, errorCode, 'EventExtendedConfigurationActionSet')

sys.exit()

[errorCode, returnString]=myxps.EventExtendedStart(socketId)

if (errorCode $!=0$ ):

self.displayErrorAndClose (socketId, errorCode, 'Event Extended Start')

sys.exit()

[errorCode, returnString]=myxps.PositionerSGammaParametersSet(socketId,positioner,int(vel),80,.005,.05)

\#[errorCode,returnString]=myxps.MultipleAxesPVTExecution(socketId,group,fileName,1)

[errorCode, returnString] $=$ myxps. GroupMoveAbsolute (socketId, group, $[$ disp] $)$

time.sleep(holdDur)

[errorCode, returnString] = myxps.GroupMoveAbsolute(socketId, group, [POC-.6])

[errorCode, returnString] $=$ myxps. GatheringStopAndSave $($ socketId $)$

if (errorCode != 0):

self.displayErrorAndClose (socketId, errorCode, 'GatheringStopandSave')

sys.exit()

def $\operatorname{smooth}($ self, $x$,window_len=10,window='flat'):

\# "'"smooth the data using a window with requested size.

\#

\#6 This method is based on the convolution of a scaled window with the signal.

\#7 The signal is prepared by introducing reflected copies of the signal 
\#8 (with the window size) in both ends so that transient parts are minimized

\#9 in the begining and end part of the output signal.

$\# 10$

\#11 input:

$\# 12 \mathrm{x}$ : the input signal

\#13 window_len: the dimension of the smoothing window; should be an odd integer

\#14 window: the type of window from 'flat', 'hanning', 'hamming', 'bartlett', 'blackman'

\#15 flat window will produce a moving average smoothing.

$\# 16$

$\# 17$ :

$\# 18$ the smoothed signal

$\# 19$

\#20 example:

$\# 21$

\#22 t=linspace $(-2,2,0.1)$

$\# 23 \mathrm{x}=\sin (\mathrm{t})+\operatorname{randn}(\operatorname{len}(\mathrm{t}))^{*} 0.1$

$\# 24 \mathrm{y}=\operatorname{smooth}(\mathrm{x})$

$\# 25$

\#26 see also:

\#27

\#28 numpy.hanning, numpy.hamming, numpy.bartlett, numpy.blackman, numpy.convolve

\#29 scipy.signal.lfilter

\#30

\#31 TODO: the window parameter could be the window itself if an array instead of a string

\#32 NOTE: length(output) != length(input), to correct this: return y[(window_len/2-1):-(window_len/2)] instead of just y.

\#33 "'"

if $\mathrm{x} . \mathrm{ndim} !=1$ :

raise ValueError, "smooth only accepts 1 dimension arrays."

if $\mathrm{x}$.size < window_len:

raise ValueError, "Input vector needs to be bigger than window size."

if window_len $<3$ :

return $\mathrm{x}$

if not window in ['flat', 'hanning', 'hamming', 'bartlett', 'blackman']:

raise ValueError, "Window is on of 'flat', 'hanning', 'hamming', 'bartlett', 'blackman'"

$\mathrm{s}=\mathrm{np} . \mathrm{r} \_[\mathrm{x} \text { [window_len-1:0:-1],x,x[-1:-window_len:-1]] }$

\#print(len(s))

if window == 'flat': \#moving average

w=np.ones(window_len,'d')

else:

w=eval('np.'+window+'(window_len)')

$\mathrm{y}=$ np.convolve(w/w.sum(),s,mode='valid')

return y 